\title{
Required Assets for a Nuclear Energy Applied R\&D Program
}

\author{
March 2009
}

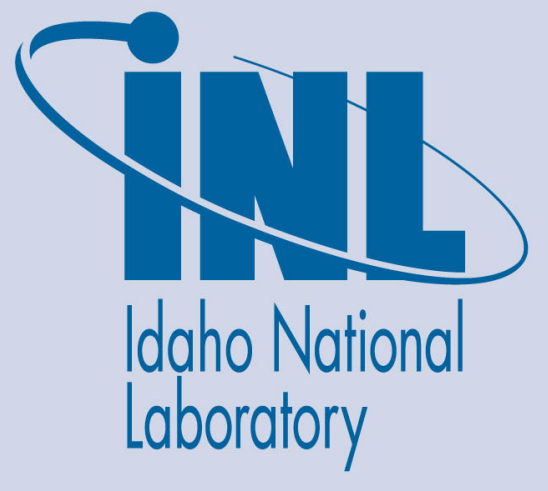

The INL is a U.S. Department of Energy National Laboratory operated by Battelle Energy Alliance 
INL/EXT-08-14092

\section{Required Assets for a Nuclear Energy Applied R\&D Program}

March 2009

\section{Idaho National Laboratory Idaho Falls, Idaho 83415}

http://www.inl.gov

Prepared for the

U.S. Department of Energy

Office of Nuclear Energy

Under DOE Idaho Operations Office

Contract DE-AC07-05ID14517 


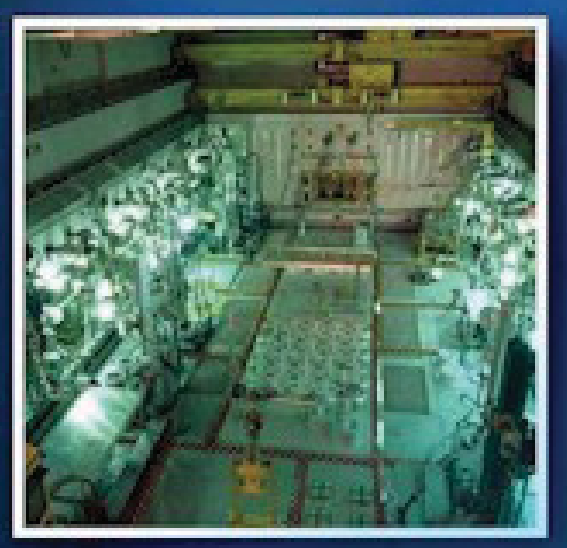

Required Assets

for a Nuclear

Energy Applied

R\&D Program
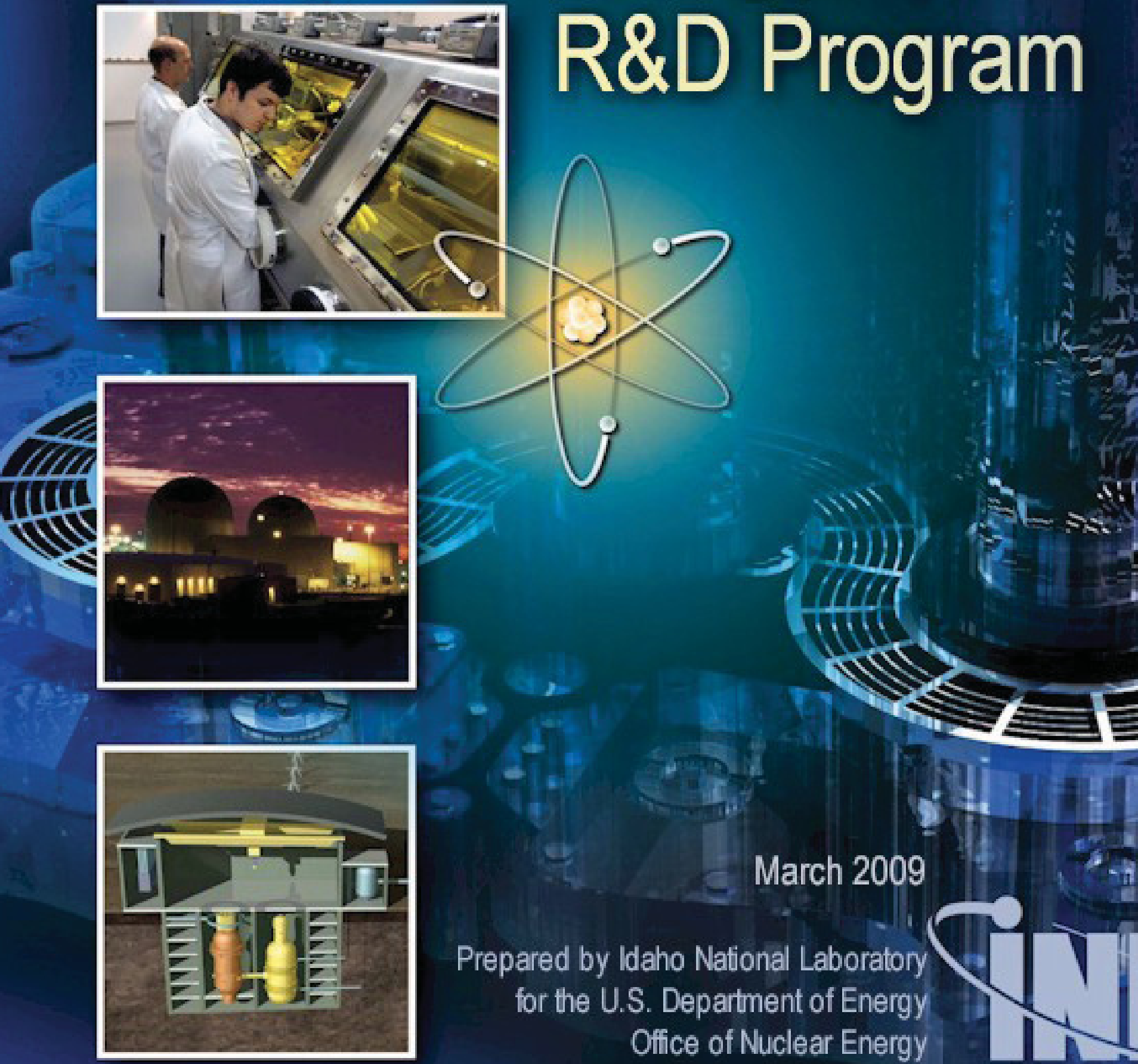


\section{DISCLAIMER}

This information was prepared as an account of work sponsored by an agency of the U.S. Government. Neither the U.S. Government nor any agency thereof, nor any of their employees, makes any warranty, expressed or implied, or assumes any legal liability or responsibility for the accuracy, completeness, or usefulness, of any information, apparatus, product, or process disclosed, or represents that its use would not infringe privately owned rights. References herein to any specific commercial product, process, or service by trade name, trade mark, manufacturer, or otherwise, does not necessarily constitute or imply its endorsement, recommendation, or favoring by the U.S. Government or any agency thereof. The views and opinions of authors expressed herein do not necessarily state or reflect those of the U.S. Government or any agency thereof. 


\section{FOREWORD}

During 2008 a number of activities were undertaken to identify the gaps in nuclear energy research and development infrastructure. This report captures three of those activities, but should be considered as only contributing to the total picture, not complete in and of itself. As with any report of this nature, there are many interesting, often related ideas, programs, facilities, projects, etc. that are not captured within its scope. Many such omitted facilities and research areas were identified during an extensive three-month international review during the last quarter of 2008. More than 150 comments and supplemental information were collected during that review. While these comments potentially would add significant value to a future study, this project is limited to the perspective that was developed in 2008.

The comments and supplemental information that were received have been included in one of two ways in this final report. The written comments have been summarized and included at the end of the appropriate section. Verbatim comments were not used in order to eliminate redundancy, reduce bulk, and to protect the privacy of the reviewers. However, the verbatim comments were displayed on the review web site during the open review period.

Several reviewers supplied supplemental information such as charts and tables that they felt should have been included in the report. Where reasonable to do so, this material has been listed in Appendix 5.3, Reviewer Supplemental Information and Attachments. No attempt has been made to validate the material; that is left to a future activity.

Since this report was first published, other related studies have been released. One report that was developed simultaneously is Nuclear Energy: Policies and Technologies for the $21^{\text {st }}$ Century, produced by the Nuclear Energy Advisory Committee for the Office of Nuclear Energy, U.S. Department of Energy. In December 2008, The Nuclear Energy Agency completed its comprehensive report on Research and Test Facilities Required in Nuclear Science and Technology. In August 2008, the Directors of the Department of Energy laboratories issued a white paper that provided direction for future research, A Sustainable Energy Future: The Essential Role of Nuclear Energy. This paper, together with the various capabilities reports provided a basis for the Office of Nuclear Energy's draft report, Facilities for the Future of Nuclear Energy Research: A Twenty-Year Outlook, which has been on the DOE-NE web site since October, 2008.

This report has not been updated from the earlier draft to account for developments in 2009. The primary change has been to acknowledge reviewer comments. Additional analysis is left to subsequent reports. 


\section{EXECUTIVE SUMMARY}

This report is one of a set of three documents that have collectively identified and recommended research and development capabilities that will be required to advance nuclear energy in the next 20 to 50 years. The first report, Nuclear Energy for the Future: Required Research and Development Capabilities-An Industry Perspective, was produced by Battelle Memorial Institute at the request of the Assistant Secretary of Nuclear Energy. That report, drawn from input by industry, academia, and Department of Energy laboratories, can be found in Appendix 5.1. This Idaho National Laboratory report maps the nuclear-specific capabilities from the Battelle report onto facility requirements, identifying options from the set of national laboratory, university, industry, and international facilities. It also identifies significant gaps in the required facility capabilities. The third document, Executive Recommendations for Nuclear R\&D Capabilities, is a letter report containing a set of recommendations made by a team of senior executives representing nuclear vendors, utilities, academia, and the national laboratories (at Battelle's request). That third report can be found in Appendix 5.2. The three reports should be considered as set in order to have a more complete picture.

The basis for this report was drawn from three sources: previous Department of Energy reports, workshops and committee meetings, and expert opinion. The facilities discussed were winnowed from several hundred facilities that had previously been catalogued and several additional facilities that had been overlooked in past exercises. The report has been extensively reviewed by international experts, the Nuclear Regulatory Commission, national laboratories, and the university community. Their comments have been summarized and displayed in the final report.

The scope of this report is limited to commercial nuclear energy and those things the federal government, or more specifically the Office of Nuclear Energy, should do to support its expanded deployment in order to increase energy security and reduce carbon emissions. In the context of this report, capabilities mean innovative, well-structured research and development programs, a viable work force, and well-equipped specialized facilities.

The report focuses on the infrastructure needed to carry out the research and development that will assure successful achievement of the following seven priorities:

- $\quad$ Extended operation of the fleet of current and future light water reactors

- $\quad$ Sustainable workforce

- $\quad$ Sustainable fuel cycle

- $\quad$ Generation-IV reactors

- $\quad$ Safeguards and security technology

- Advanced modeling and simulation

- $\quad$ Space and defense power systems (not included in the Battelle reports). 
Analyzing the infrastructure needed to address these research and development priorities involved identifying the generic capabilities needed, listing the existing facilities that could be applied, evaluating the state of the relevant facilities, and looking for major gaps between current capabilities and future needs. The following major conclusions can be drawn from this assessment:

- In spite of the age of the existing infrastructure, it can serve much of the intended purpose in the near term provided that necessary modifications, upgrades, equipment additions, and sustained regular maintenance are made. This will require substantial investment, perhaps proportionally similar to investments made by nuclear utilities for license extensions and power uprates.

- Japan, France, the United Kingdom, and Russia have capabilities that are not available in the United States. Although it would require a change in operating mode, some of these international facilities potentially could be used in a collaborative arrangement on an interim basis until the United States reestablishes its nuclear research infrastructure.

- Development of a viable nuclear workforce that includes top quality researchers will require the Department of Energy to resume stewardship of higher education in nuclear engineering that is consistent and adequately funded.

- There are several notable gaps in currently available infrastructure. A licensed prototype hightemperature reactor is needed to demonstrate the broad applicability of nuclear energy to manufacturing, refining, and hydrogen production in addition to electricity generation. A collective set of capabilities for developing the technologies needed for a sustainable fuel cycle is needed. These capabilities could be put together in several ways, ranging from piecemeal existing facilities to new consolidated construction. A fast reactor prototype or test facility also is needed to develop the technology that will be commercialized in mid-century.

- $\quad$ Modeling and simulation of reactor systems will play an increasingly important role as higher performance computers and advanced software will enable simulations that were previously impossible. Given the enormous complexity of power plants during short transients, normal operation, fuel lifetime, and plant lifetime, the principal benefit of advanced computing will be verification of specific effects that are modeled in applied systems codes and accelerating the development of new systems through fewer and better designed experiments. A crucial and urgent element is retrieval and archiving of irreplaceable legacy data. Facility requirements for advanced modeling and simulation include a supercomputing facility with support structure, special effects laboratories, and a knowledge preservation center.

- The Department of Energy will need a structured process for dealing with facility limitations, particularly in the near term. An active research and development program will demand more capabilities than are currently available. As primary facilities are filled to capacity, either delays will need to be scheduled or programs will expand into secondary facilities or both. When that approach is no longer sufficient, use of international facilities and construction of new facilities will be required.

This report and the Battelle reports are based on a linear unfolding of research and development needs. They do not attempt to identify how innovation or major policy changes could affect future capability requirements. 


\section{CONTENTS}

FOREWORD

EXECUTIVE SUMMARY iv

ACRONYMS

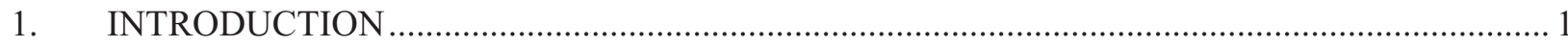

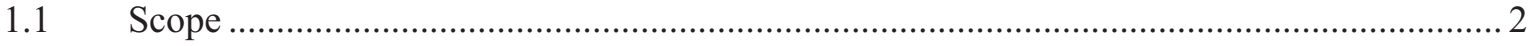

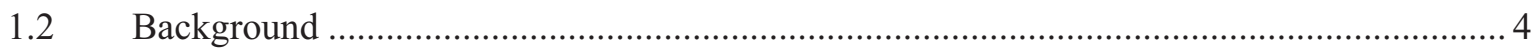

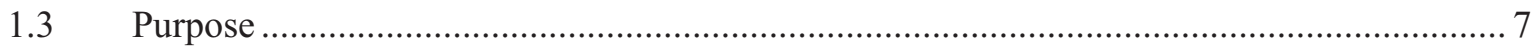

2. FACILITY OPTIONS FOR MEETING R\&D REQUIREMENTS …........................................ 7

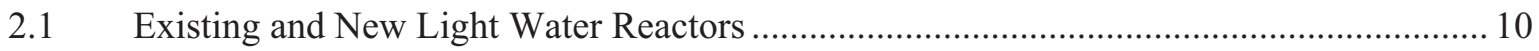

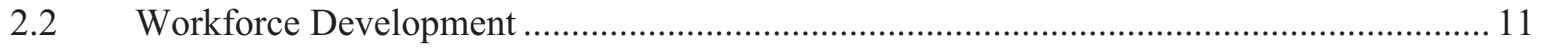

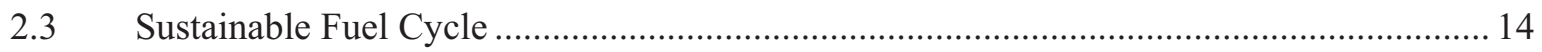

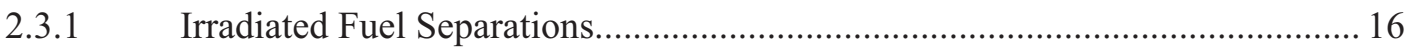

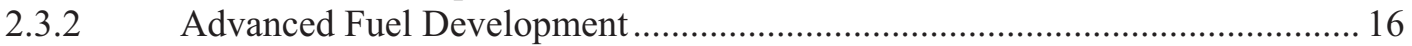

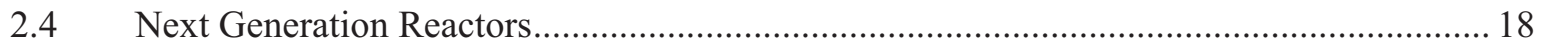

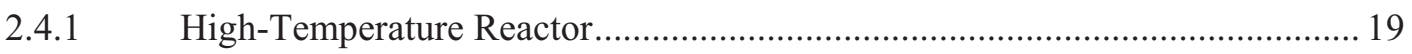

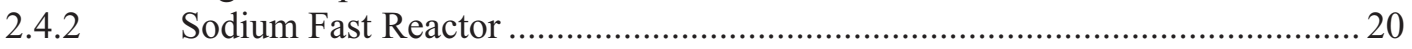

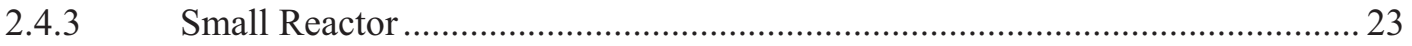

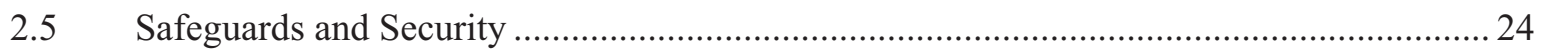

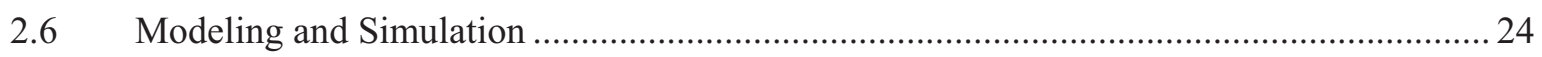

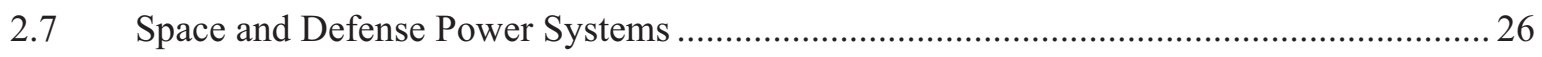

3. DOMESTIC AND INTERNATIONAL NUCLEAR R\&D ASSETS …...................................... 28

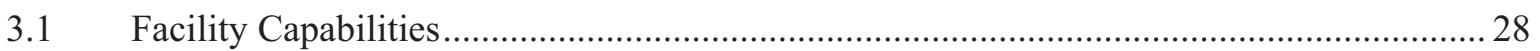

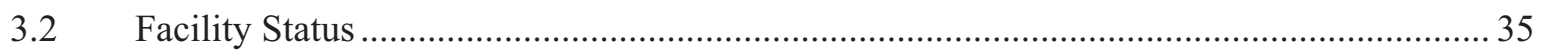

3.2.1 Facility Assessment Stoplight Color-Coded Rating System .............................36

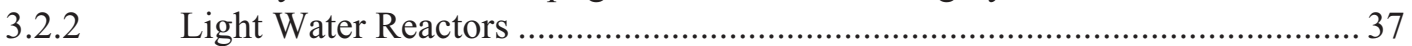

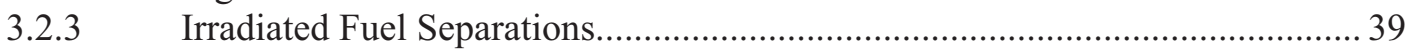

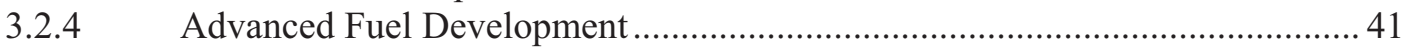

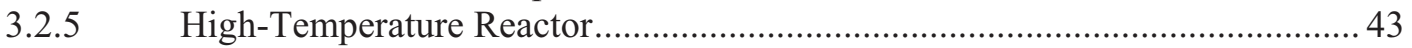

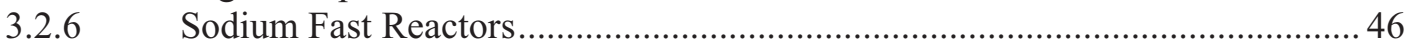

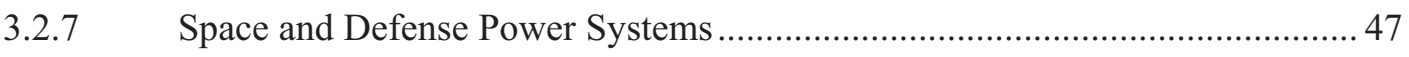




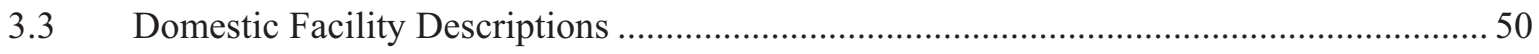

3.3.1 Department of Energy National Laboratories …........................................ 50

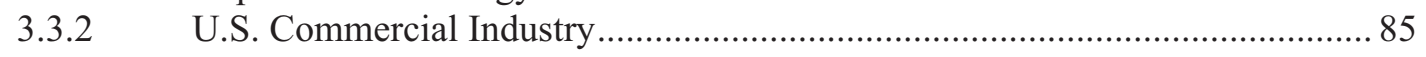

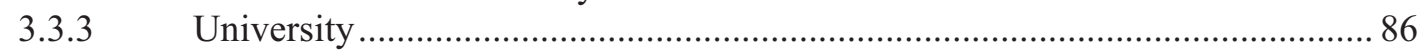

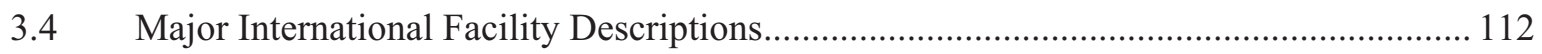

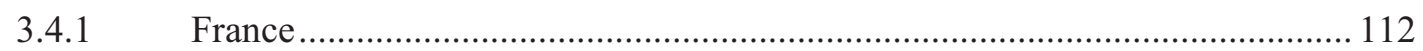

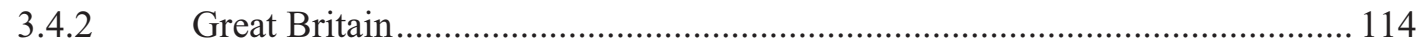

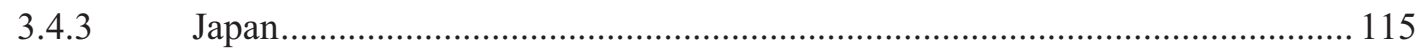

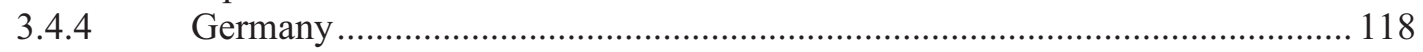

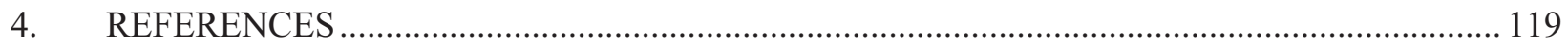

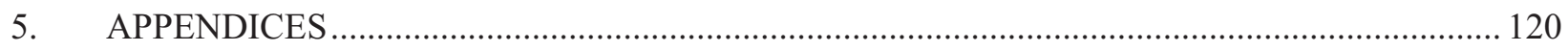

5.1 Nuclear Energy for the Future: Required Research and Development Capabilities—An

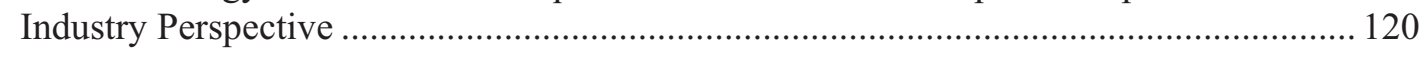

5.2 Executive Recommendations for Nuclear Research and Development Capabilities ........ 172

5.3 Reviewer Supplemental Information and Attachments............................................. 183

5.3.1 Supplemental Information Included in this Report ....................................... 183

5.3.2 Supplemental Information Submitted and Filed for Future Consideration ..... 183

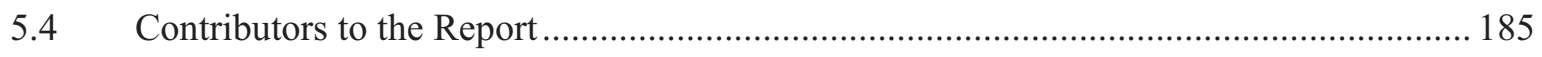

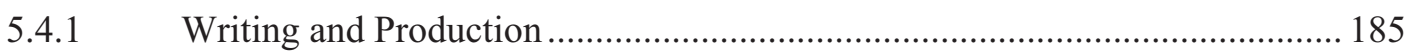

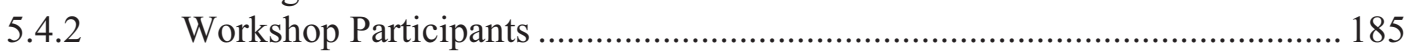

5.4.3 National Research and Development Directors ............................................ 185

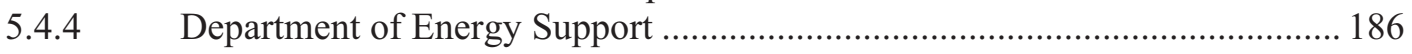




\section{FIGURES}

Figure 1-1. The required research and development capabilities report produced by Battelle complements this required assets report.

Figure 1-2. Some Department of Energy nuclear facilities face difficult challenges in being retrofitted and upgraded for new nuclear missions.

Figure 1-3. The Department of Energy Office of Nuclear Energy intends to produce a research and development infrastructure plan along the lines of the Office of Science's 20-year facility outlook. 6

Figure 1-4. Previous compilations of Department of Energy facilities that could be used for nuclear energy research and development were consulted in the development of this required assets report. 6

Figure 2-1. The British Technology Centre is a new facility with advanced fuel cycle development capabilities. 8

Figure 2-2. Commercial industries which construct and operate light water reactors will require access to specialized facilities and research personnel in the DOE complex.

Figure 2-3. The University of Wisconsin is one of two dozen major universities that are turning out the future nuclear research and development workforce.

Figure 2-4. The MIT reactor is involved in Department of Energy research and development programs. 13

Figure 2-5. Expanded use of Department of Energy assets, such as the Advanced Test Reactor National Scientific User Facility, will supplement university programs and capabilities.

Figure 2-6. Artist's conception of a new consolidated Advanced Fuel Cycle Facility. The Advanced Fuel Cycle Facility's capabilities may actually be distributed among upgraded existing facilities........ 14

Figure 2-7. The sodium-cooled fast reactor Phenix has served French research and development programs for decades. The Department of Energy currently has advanced fuel experiments under irradiation in Phenix.

Figure 2-8. The 335-MWe IRIS design by Westinghouse is an example of a small light water reactor that could be used for regional deployment.

Figure 2-9. Supercomputers have high demand for power, cooling, and cleanliness that requires specialized facilities.

Figure 2-10. National Aeronautics and Space Administration supply of plutonium-238 for solar system exploration will be exhausted in the year 2015 .

Core of the Neutron Radiography Facility at the Idaho National Laboratory 


\section{TABLES}

Table 1-1. Summary of the facility stoplight color-coded rating system................................................ 3

Table 1-2. Some previous compilations of nuclear energy research assets. .......................................... 5

Table 2-1. Facilities considered for support of light water reactor research and development. ................ 11

Table 2-2. Existing facilities evaluated by the Department of Energy for support of major Advanced Fuel

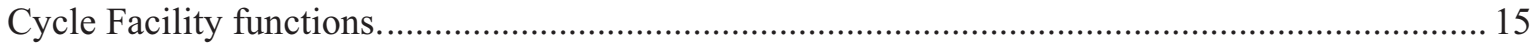

Table 2-3. Existing facilities considered for support of advanced fuel development. .............................. 17

Table 2-4. Existing facilities considered for support of Next Generation Nuclear Plant research and

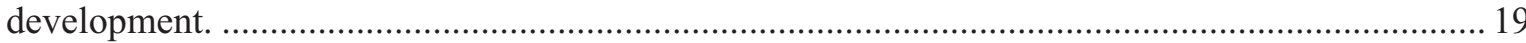

Table 2-5. Existing facilities considered for support of sodium-cooled fast reactor research and

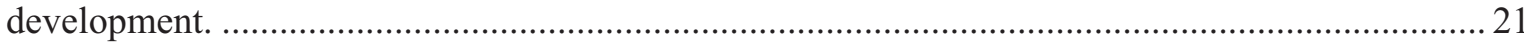

Table 2-6. Facilities considered for support of plutonium-238 production, processing, and handling.......27

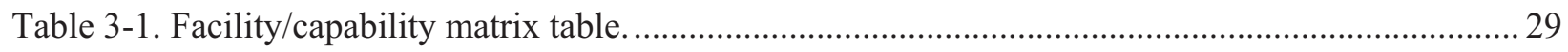

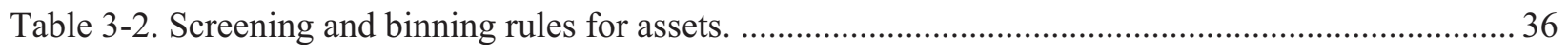

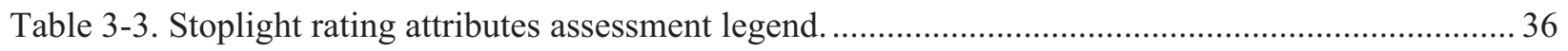

Table 3-4. Summary example of the facility stoplight color-coded rating system. ................................ 38

Table 3-5. Facilities assessed for light water reactor research and development support. ........................ 38

Table 3-6. Summary example of the facility stoplight color-coded rating system. ................................. 39

Table 3-7. Facilities assessed for irradiated fuel separations research and development support. ............. 39

Table 3-8. Summary example of the facility stoplight color-coded rating system. ................................. 41

Table 3-9. Facilities assessed for advanced fuel development research and development support........... 41

Table 3-10. Summary example of the facility stoplight color-coded rating system. ............................... 44

Table 3-11. Facilities assessed for high-temperature reactor research and development support. ............. 44

Table 3-12. Summary example of the facility stoplight color-coded rating system. .............................. 46

Table 3-13. Facilities assessed for sodium fast reactor research and development support. .................... 46

Table 3-14. Summary example of the facility stoplight color-coded rating system. .............................. 48

Table 3-15. Facilities assessed for space and defense power systems research and development support. 48 


\section{ACRONYMS}

ACRR Annular Core Research Reactor

AFCF Advanced Fuel Cycle Facility

ATR Advanced Test Reactor

CEA Commissariat à l'énergie atomique

DOE U.S. Department of Energy

EBR-II Experimental Breeder Reactor-II

FASF Flourinel Dissolution Process and Fuel Storage Facility

FCF Fuel Conditioning Facility

FFTF Fast Flux Test Facility

GFR gas-cooled fast reactor

HFEF Hot Fuel Examination Facility

JAEA Japan Atomic Energy Agency

LAMDA Low-Activation Materials Development and Analysis Laboratory

LFR lead-cooled fast reactor

LWR light water reactor

MITR Massachusetts Institute of Technology Reactor

MSR molten salt reactor

NE Office of Nuclear Energy

NGNP Next Generation Nuclear Plant

NNSA National Nuclear Security Administration

NOG B\&W Nuclear Operations Group

$\mathrm{R} \& \mathrm{D} \quad$ research and development

SCWR supercritical water reactor

SFR sodium-cooled fast reactor

TREAT Transient Reactor Test Facility

VHTR very high-temperature reactor 


\section{Required Assets for a Nuclear Energy Applied R\&D Program}

\section{INTRODUCTION}

The future of nuclear power is an important consideration in the national agenda to transform from our current fossil fuel dependency to a secure, clean, diverse energy supply. Nuclear energy is a stable domestic resource that produces $20 \%$ of the United States electricity, accounting for at least $70 \%$ of all emission-free generation. With readily expandable baseload capacity, nuclear electricity can help stabilize transmission infrastructure as intermittent wind and solar capacity are added to the grid at a significant level. Nuclear technology is now widely recognized by world leaders as one of the major tools for supply-side carbon abatement - a massive undertaking needed to limit climate change to an acceptable level.

The 104 operating United States power reactors lead the world in nuclear electricity production with 800 billion kilowatt hours per year, about $45 \%$ more than is generated by the second-place fleet of French nuclear plants. But with $80 \%$ nuclear electricity consumption, France's economy is one of the least carbon-intensive in the world - less than half that of the United States, which relies on $70 \%$ fossil energy for generating electricity. Without nuclear generation, the United States would emit an additional 700 million tons of carbon dioxide annually.

As with every energy technology deployed at industrial scale, significant federal research and development (R\&D) preceded operation of the current nuclear power plants. For the 40 years before the last of the Generation-II reactors went online in the early 1990s, the U.S. government invested about $\$ 60$ billion in nuclear R\&D. For the remainder of the century, nuclear R\&D was sharply diminished because of a policy assumption that existing U.S. reactors would operate for 40 years, shut down, and no more would be built. That position was reversed with the passage of the Energy Policy Act of 2005, which authorized future nuclear R\&D and provided incentives for construction of new clean energy generation, including nuclear.

Energy planners foresee nuclear energy's role at mid-century to range from 16 to $50 \%$ of total United States electrical generation, depending on how aggressively we attack carbon emissions. The latest Energy Information Administration outlook projects a 21\% growth in electricity over the next 20 years, with a $14 \%$ growth in nuclear electric generation. Even at the low end of the range, aggressive investment in existing plants and construction of new Generation-III nuclear plants will be needed. Most of the required investment will be made by the nuclear industry, an ongoing effort to uprate existing plants; extend operating licenses from 40 to 60 years; and design, construct, and license Generation-III nuclear plants. There also will be extensive leveraging of technologies developed for non-nuclear applications.

Nevertheless, the tens of billions of dollars expected to be invested by the nuclear industry will be insufficient to sustain a United States nuclear renaissance. There is still an essential federal government role that includes control of nuclear materials, assuring nuclear nonproliferation abroad, ultimate management of used nuclear fuel, and R\&D that is beyond the capability of the commercial industry. Such R\&D includes understanding how materials age over decades of service at temperature in an irradiation environment - perhaps enabling current technology to remain in service beyond 60 years; developing a sustainable fuel cycle system that comprises fuel recycling, advanced reactors, robust waste forms, and a geologic repository; developing a high-temperature reactor for emission-free industrial heat process applications; and developing safe, lightweight nuclear power systems that enable solar system exploration. The U.S. Department of Energy (DOE) is currently evaluating the human capital and 
infrastructure capabilities needed to conduct the required research, development, and demonstration during the next quarter century.

\section{Summary of Reviewer Comments}

1. The report does not consider R\&D for the gas-cooled fast reactor.

2. Application of modern modeling and simulation techniques should be made to sound more revolutionary.

3. Facilities to measure basic nuclear data or to provide integral benchmarking for radiation transport calculations are not included.

4. Discussion of human factors, digital instrumentation and equipment, reliability, availability

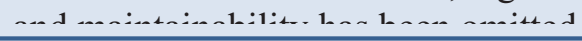

\subsection{Scope}

This draft report presents a high level view of the infrastructure requirements for a revitalized United States nuclear energy R\&D program. Previous DOE reports, independent reports, workshops, and expert opinions provided the basis for this report.

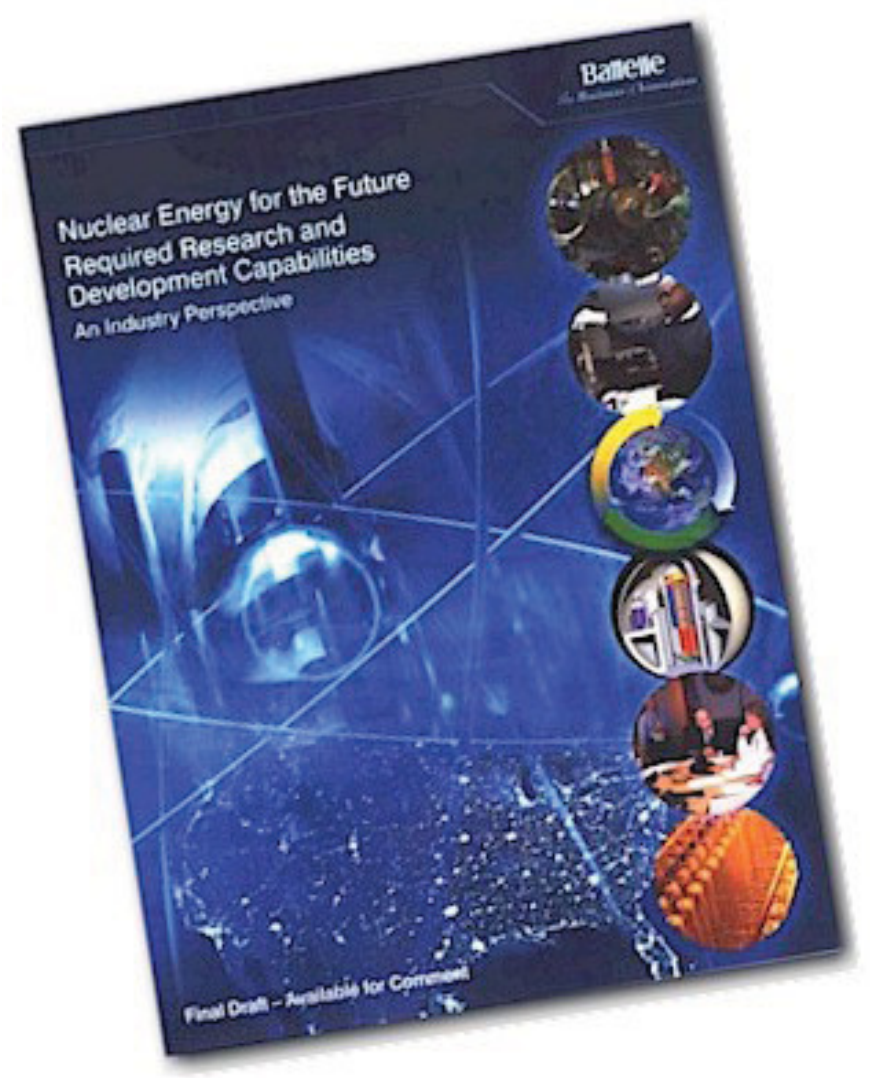

Figure 1-1. The required research and development capabilities report produced by Battelle complements this required assets report.
The scope of this report comprises the identification of options for the infrastructure capabilities needed to effectively carry out the projected R\&D of a vibrant nuclear energy program in the first half of the twenty-first century. The report's scope is bounded by the responsibilities of the DOE Office of Nuclear Energy (NE). In draft form, the report contains industry-validated $\mathrm{R} \& \mathrm{D}$ needs for the next 20 to 50 years. A parallel effort led by the Battelle Memorial Institute collected these requirements in a companion report, Nuclear Energy for the Future: Required Research and Development Capabilities-An Industry Perspective (see Appendix 5.1). The reports are meant to be read as a set, together with the ensuing recommendations (see Appendix 5.2) from a Battelle-assembled team of senior executives representing industry, academia, and the national laboratories.

In identifying facilities needed to execute a multidecade R\&D program, the unrestricted solution would be new, $21^{\text {st }}$-century infrastructure that is suited 
to the task and capable of drawing and retaining new generations of nuclear scientists and engineers. Because neither timing nor budgets will likely allow this approach across the R\&D portfolio, the report focuses primarily on existing domestic and international facilities that, with appropriate modifications, could perform many of the required tasks.

At the top level of the existing infrastructure options are the DOE-owned facilities and capabilities within the complex of national laboratories. At the next level, there are limited physical capabilities within universities and the domestic nuclear industry. Finally, there is an important class of international assets that may be accessible through international collaboration and agreements.

This report has two main parts. Section 2 maps industry-validated goals, such as "sustainable fuel cycle," to the major infrastructure needed to successfully carry out the required R\&D. The justification of these goals is left to the Battelle report. Section 3 maps available and proposed facilities to the Battelle-indentified generic resource requirements, such as hot cells and test reactors. For most existing domestic facilities, Section 3 also provides a color-coded rating of six key decision parameters: condition, capability, availability, regulatory status, safeguards and security, and human capital. A summary of the rating system is shown in Table 1-1.

Table 1-1. Summary of the facility stoplight color-coded rating system.

\begin{tabular}{|l|l|}
\hline Green & Mission ready \\
\hline Yellow & Ready within 2 years or less with modest investment \\
\hline Orange & Needs modification or upgrades that require significant investment \\
\hline Red & Usable only after major capital investment over an extended number of years \\
\hline
\end{tabular}

Unlike previous reports listing hundreds of nuclear-capable assets that might be used at some point in the future, this report has taken the data from those previous exercises, screened them, and binned them into a manageable list of major facilities. Each of these major facilities is described on a single page, with applicability to specific programs identified. Capabilities at universities, within U.S. industry, and in overseas nuclear development programs are listed, but not evaluated in detail.

The scope of this report is limited to commercial nuclear energy and those things the federal government should do to support its expanded deployment in order to increase energy security and reduce carbon emissions. Consequently, the report does not address the production of industrial and medical isotopes, the use of nuclear energy for space exploration, or the development of a geologic repository. Nor does the report address the capabilities provided by the commercial nuclear industry, such as low-level waste management. Within its scope, this report does not recommend a specific set of facilities; this is the responsibility of DOE through its National Environmental Policy Act and project management order processes. In the context of this report, capabilities mean innovative, well-structured R\&D programs, a viable work force, and well-equipped specialized facilities. 


\section{Summary of Reviewer Comments}

1. The discussion of 50-year-old facilities could leave readers with the incorrect impression that they are unsafe for research today.

2. Neutron scattering research should be added.

3. Advanced reactors will have novel issues that will require sophisticated computational resources and good thermal hydraulic experimental facilities.

4. $\mathrm{Pu}-238$ production is considered, but not medical isotopes.

\subsection{Background}

From the 1950s through the 1970s, the United States invested heavily in a new class of infrastructure. The interstate highway system was constructed, the National Aeronautics and Space Administration assembled the capability to explore the moon and solar system, and the Atomic Energy Commission built an unprecedented scientific and engineering research infrastructure. Spanning the continent, the Atomic Energy Commission's national laboratories dominated nuclear energy development and big science. During this era, the technical basis for a commercial nuclear industry was established, the first generation nuclear power plants were operated, fast reactors were built and operated successfully, and defense nuclear reprocessing was conducted at a scale that dwarfs global commercial capacity today.

The surviving physical infrastructure that was built in that era comprises most of DOE's nuclear energy assets today. Major capabilities, such as the Fast Flux Test Facility (FFTF) and the Experimental Breeder Reactor-II (EBR-II), have been shut down as a result of policy decisions made in the 1990s. The utility of the surviving facilities ranges from pending demolition to robustly active. In general though, the federal facilities have not benefitted from the sustained maintenance and upgrading that has enabled vastly improved operation of commercial nuclear power plants.

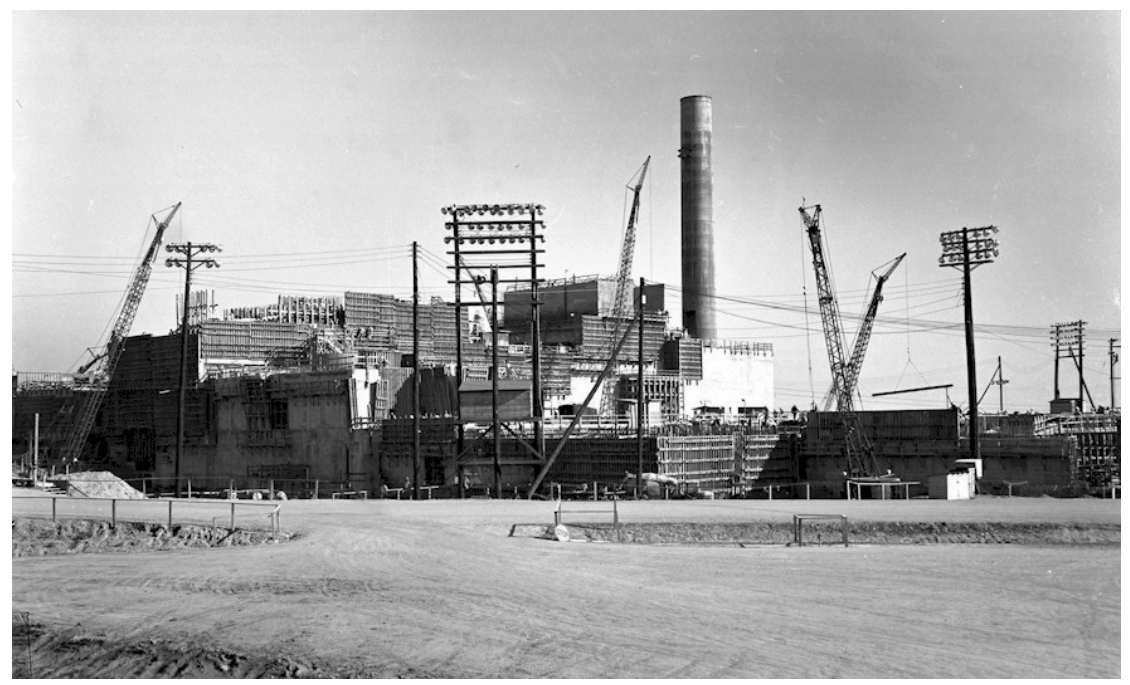

Figure 1-2. Some Department of Energy nuclear facilities face difficult challenges in being retrofitted and upgraded for new nuclear missions.

Built in a less demanding era, existing DOE nuclear facilities face difficult challenges in being retrofitted and upgraded for new nuclear missions. Their capital benefit is in concrete and steel, which are 
now expensive commodities. While a high safety standard is applied to existing DOE facilities, most 50year old facilities unfortunately do not have the quality assurance pedigree needed for high-source-term nuclear work today. Extensive qualification and retrofitting will be required if these assets are to be used effectively in a modern R\&D program. The following list shows some of the challenges:

- $\quad$ Tightened seismic requirements will require extensive analysis followed by physical upgrades such as enhanced bracing, new ventilation systems, new fire protection, and anchoring for heavy equipment.

- Encroachment by other site activities and the surrounding community can make it difficult to meet modern requirements for a safety or security buffer zone.

- A structure can be in reasonable physical condition, but vital site infrastructure, such as power, water, sewer, office facilities, waste management facilities, shipping and receiving, and security, can be degraded or missing.

- National Environmental Policy Act documentation is required for new missions.

- $\quad$ Few facilities currently meet the emerging DOE security requirements for protecting nuclear materials. Without an existing security base, site modifications to meet these requirements could be prohibitively expensive for some levels of development and demonstration operations.

- $\quad$ New safety envelopes will be necessary with commensurate safety equipment upgrades.

Taken alone, few of these challenges are likely to be fatal for existing facilities. The commercial nuclear industry has set a high standard in extracting value from mature facilities. However, the cumulative cost of overcoming several of these barriers will turn some potentially useful federal facilities into improbable options. Those decisions can only be made after DOE goes through its environmental and engineering processes. These processes are required by DOE Order 413.3, "Program and Project Management for the Acquisition of Capital Assets."

DOE has chartered previous studies of facilities that could be used in its nuclear energy R\&D programs. Table 1-2 contains a list of some key reports.

Table 1-2. Some previous compilations of nuclear energy research assets.

\begin{tabular}{|c|c|c|}
\hline Year & Title & Purpose \\
\hline 2003 & $\begin{array}{l}\text { Report of the Infrastructure Task Force of the DOE } \\
\text { Nuclear Energy Research Advisory Committee }\end{array}$ & $\begin{array}{l}\text { Advise the Nuclear Energy Research Advisory Committee } \\
\text { about what characteristics, capabilities, and attributes a } \\
\text { world-class nuclear laboratory would possess }\end{array}$ \\
\hline 2005 & $\begin{array}{l}\text { Nuclear Facility Consolidation and Modernization Task } \\
\text { Force Report - A Contractor Initiated Study }\end{array}$ & Nuclear infrastructure assessment and recommendations \\
\hline 2006 & $\begin{array}{l}\text { Hot Cells Strategy to Advance Nuclear Science and } \\
\text { Engineering }\end{array}$ & $\begin{array}{l}\text { An inventory and capabilities assessment of } \\
\text { Battelle-operated hot cell facilities }\end{array}$ \\
\hline 2006 & $\begin{array}{l}\text { DOE Complex-Wide Capability Report: Nuclear Energy } \\
\text { Infrastructure }\end{array}$ & $\begin{array}{l}\text { Compilation of civilian nuclear facilities and capabilities } \\
\text { required by the Energy Policy Act of } 2005\end{array}$ \\
\hline 2007 & $\begin{array}{l}\text { Inventory of Civilian Nuclear Facilities, Equipment, and } \\
\text { other Assets }\end{array}$ & $\begin{array}{l}\text { Update of the facilities and assets inventory section of the } \\
2006 \text { Complex-wide Capability Report }\end{array}$ \\
\hline 2008 & AFCF Existing Facilities Data Report (draft) & $\begin{array}{l}\text { Assesses facilities for potential future support of } \\
\text { Advanced Fuel Cycle Initiative R\&D }\end{array}$ \\
\hline
\end{tabular}




\begin{tabular}{|l|l|l|}
\hline Year & Title & Purpose \\
\hline 2008 & $\begin{array}{l}\text { U.S. Facilities Needed to Support Global Nuclear Energy } \\
\text { Partnership Research and Development (draft) }\end{array}$ & $\begin{array}{l}\text { Identifies Global Nuclear Energy Partnership R\&D facility } \\
\text { needs }\end{array}$ \\
\hline 2008 & $\begin{array}{l}\text { SFR-Related Facilities (United States, France, and } \\
\text { Japan) DRAFT }\end{array}$ & $\begin{array}{l}\text { Indentifies facilities in the three countries that potentially } \\
\text { could be shared for sodium fast reactor research }\end{array}$ \\
\hline
\end{tabular}

In general, the effort that went into some of the earlier compilations has not been rewarded with an enduring product. One reason is the sheer volume of data that were compiled. The databases were simply too unwieldy to be directly useable in decision making. Furthermore, the data were inconsistent from site to site. Sorting, binning, normalizing, and validating the data would have required substantially more effort, time, and cost.

One exception to low utility of the previous reports is the current draft AFCF Existing Facilities Data Report (2008). The Advanced Fuel Cycle Facility (AFCF) report is focused on one specific need. The evaluation team visited each of the prospective facilities in order to do a consistent assessment. The report proved to be useful for this exercise.

Two other draft documents that are simultaneously being developed by DOE are directly pertinent and to some extent redundant with this exercise. The first is the draft report on U.S. Facilities Needed to Support Global Nuclear Energy Partnership Research and Development. The output of this report was described in detail to the Nuclear Energy Advisory Committee, Subcommittee on Facilities, on June 16 and 17,2008 . The second document (SFR-Related Facilities), which is in an early draft stage, is a table of facilities in France, Japan, and the United States that potentially could be jointly used in developing sodium fast reactor technology under a current trilateral agreement.
Figure 1-3. The Department of Energy Office of Nuclear Energy intends to produce a research and development infrastructure plan along the lines of the Office of Science's 20-year facility outlook.
Figure 1-4. Previous compilations of Department of Energy facilities that could be used for nuclear energy research and development were consulted in the development of this required assets report. 
A recent development is the specification of facility requirements by the major program lines within the DOE NE for the Nuclear Energy Advisory Committee. The facility specifications have different levels of maturity, but collectively they form an excellent starting point for evaluating DOE's nuclear facility needs for the next 20 to 50 years. These programmatic evaluations have been used extensively in this report because they represent the most up-to-date and comprehensive information available.

\section{Summary of Reviewer Comments}

1. All assets used in support of new reactor development must comply with applicable industry and Federal Government quality assurance requirements.

\subsection{Purpose}

DOE-NE intends to use this report as one interim step in producing an R\&D infrastructure plan along the lines of the DOE Office of Science report, Facilities for the Future of Science: A 20 Year Outlook. The industry-validated R\&D requirements that Battelle compiled and evaluated was the essential first step in producing the DOE-NE facility plan. Because of the timing of the production of these reports, the DOE-NE report will likely be used initially to advise the transition team for the next Administration. The purpose of this report is to provide specific facility options. There are significant gaps in knowledge of future budget outlays, the cost of the various options, the eventual success of the trilateral agreement with France and Japan in terms of providing realistic international options, and how prioritization will influence sequencing of facility projects. The recommendations necessarily reflect these uncertainties.

\section{FACILITY OPTIONS FOR MEETING R\&D REQUIREMENTS}

With two exceptions, the requirements discussed below map directly from the nuclear energy specific requirements in Battelle's report, Nuclear Energy for the Future: Required R\&D Capabilities-

an Industry Perspective. The first exception is regulatory $\mathrm{R} \& \mathrm{D}$, which has not been sufficiently defined to identify DOE facility needs. The second exception is space and defense power systems, which was not of interest to the segments of the industry that Battelle interviewed.

The information on requirements is obviously tied to current program planning within DOE NE. However, informed recommendations also must take into account the advice that DOE has received from the National Research Council, the General Accounting Office, and Congress. There has been a mix of positive and negative feedback, but to date criticism has been consistent in discouraging moving rapidly ahead with large commercial-scale facilities. Because such facilities will necessarily be designed, built, and operated by commercial entities, they would be outside the scope of this report. The positive recommendations from these organizations align well with the Battelle recommendations.

Recommendations for Planning Major New Facility Investments: From the recent reviews conducted by Battelle, DOE-NE's program leads, the Nuclear Energy Advisory Committee, and Idaho National Laboratory, it is clear that substantial infrastructure investment will be required for a robust nuclear science and technology R\&D program. From those reviews, four major new R\&D capabilities that will require substantial investment should be planned:

- A prototype high-temperature reactor

- $\quad$ An advanced fuel cycle facility 
- A prototype fast reactor or fast test reactor

- $\quad$ An advanced computing facility

The design, location, timing, and priority of these four major facility capabilities will have to be developed during the next Administration. It will likely be necessary to have a phased approach to developing these capabilities. Substantial investment in existing facilities may suffice to develop some of these capabilities.

\section{General Discussion:}

- $\quad$ Because of annual funding constraints, new construction and facility upgrades will require wellplanned sequencing and justification. In addition to the initial cost, annual maintenance and operations costs, support infrastructure, implementation of security requirements, and transportation infrastructure also must be addressed.

- A key recommendation from industry is to support workforce development. Participation in major DOE infrastructure projects is an excellent way for skilled workers at all levels to gain relevant experience. More importantly, world-class infrastructure helps attract top students into the nuclear science and technology R\&D arena. In addition to nuclear engineers, other disciplines (such as chemists, mechanical engineers, electrical engineers, chemical engineers, materials scientists and physicists) are needed for the broad range of nuclear R\&D for which DOE is responsible.

- $\quad$ There is tension between DOE's goals to improve international and university collaboration in significant research projects and its need to implement a pending step change in security requirements. In making facility decisions, significant inventories of controlled materials will be limited to a few hardened locations. Other laboratory locations should be planned for easier access to students and foreign collaborators, whether "outside the fence" or at a different site.

- $\quad$ There is a great deal of work, such as engineering cost studies and environmental assessments, that must be done in examining facility alternatives before down selecting to a particular option. DOE has made a good start in examining domestic options for its AFCF by having a dedicated team evaluate options against specific criteria.

- $\quad$ During its deliberations, the Nuclear Energy Advisory Committee has commented that DOE-NE should consider evaluating the potential applicability of the Office of Science model for participating in major international experiments. For advanced nuclear energy $R \& D$, there could be significant savings by sharing facility assets with international partners under existing agreements. Initially, this option could be considered for fast reactor and fuel cycle research with Japan and France, both of which have major capabilities that are not available in the United

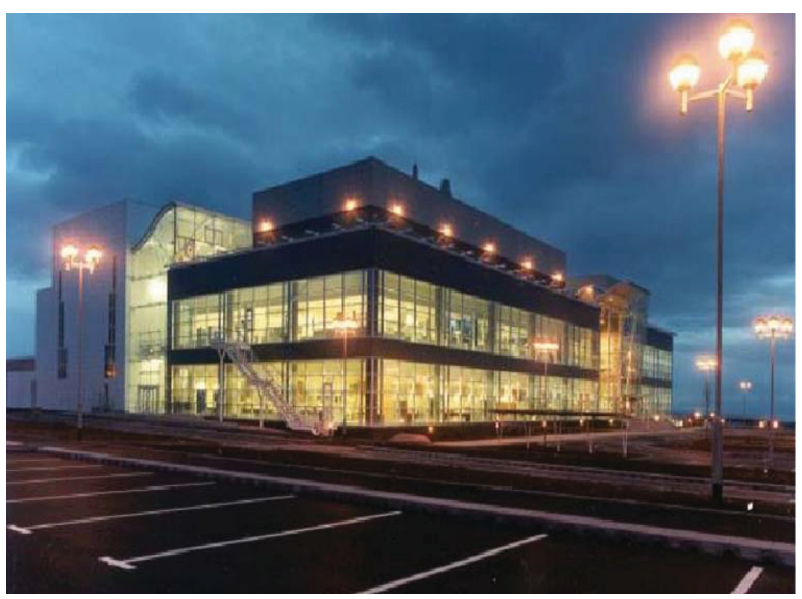

Figure 2-1. The British Technology Centre is a new facility with advanced fuel cycle development capabilities.

States. In planning future U.S. 
facilities, consideration should be given to complementing international partners' existing facilities and plans rather than building a complete infrastructure in the United States.

- This report focuses on long-term nuclear R\&D needs, as opposed to the top industry priority of new plant builds, and DOE acceptance of the used fuel at the reactor sites. The industry priority for extending the life of operating plants beyond 60 years is addressed.

- Close coordination with the Nuclear Regulatory Commission on R\&D issues is required for licensing new reactors and fuel cycle plants.

- International experiments must be conducted for codes (reactor physics, thermal-hydraulics, severe accidents, etc.) verification and validation.

- $\quad$ Some emerging research needs do not require a secure national laboratory infrastructure because they do not involve nuclear materials that must be controlled at great expense. An example is some types of engineering development work that is needed on digital, mechanical, and thermal systems. A portion of this type work can be done at universities.

- A cross-cutting theme for research needs is advanced modeling and simulation capabilities that are developed at the DOE laboratories and universities. Such capabilities could find important application in all program areas.

\section{Summary of Reviewer Comments}

1. The need for an advanced computational facility is overstated.

2. There is no mention of critical facilities.

3. The British Technology Centre does not yet have radiological capabilities.

4. International experiments must be conducted for code verification and validation.

5. Uranium enrichment capability is not discussed.

6. The university facility descriptions should have more detail for balance with the national laboratory facilities.

7. It should be noted that supercomputers become obsolete in 5-7 years. 


\subsection{Existing and New Light Water Reactors}

Industry and DOE have confirmed a high priority need to support the continued operation of the 104 existing U.S. commercial reactors and the new plants that will be constructed and operated in the next 20 years. Virtually all the existing reactors are expected to have license extensions that will allow them to operate for 60 years. Because they are significant economic, security, and carbon abatement assets, it would be highly desirable for light water reactors (LWRs) to continue operating after 60 years of service if they are safe and reliable.

Satisfying many of the requirements for extending the lifetime of operating LWRs will be the purview of the commercial industry. However, some activities will require access to specialized scientific and engineering facilities in the DOE complex, and will require research personnel who can address the relevant issues. A joint public-private program to support the LWR extended-life objective could have a significant positive impact on the United States' energy security.

Materials aging issues dominate the spectrum of research challenges for 80-year operation of LWRs. Sufficiently understanding materials aging issues for very long-term operation will require the use of DOE and university laboratories and expertise.

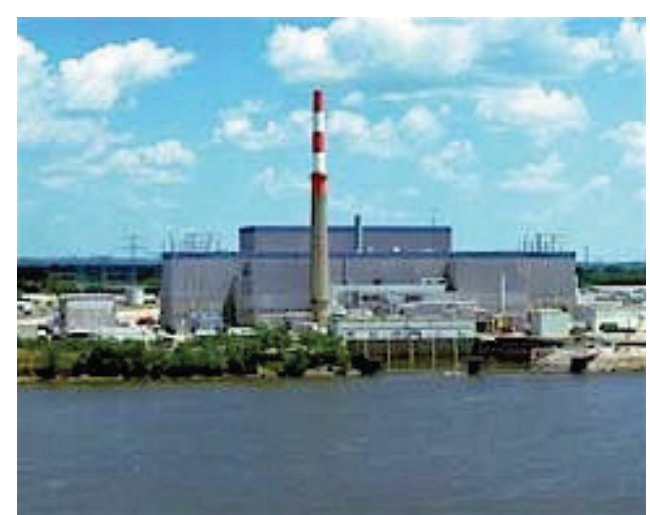

Figure 2-2. Commercial industries which construct and operate light water reactors will require access to specialized facilities and research personnel in the DOE complex.

The second major area of research that requires breakthrough technology is very long-lived LWR fuel. Global suppliers of nuclear fuel are continuously developing evolutionary improvements to LWR fuel, having increased average burnup from about $33 \mathrm{MWd} / \mathrm{kg}$ to more than $50 \mathrm{MWd} / \mathrm{kg}$, with goals extending to at least $70 \mathrm{MWd} / \mathrm{kg}$. However, design and qualification of fuel capable of greater than $100 \mathrm{MWd} / \mathrm{kg}$ will require alternative designs and claddings. This type of high-risk research is best carried out with federal assistance. Among the benefits would be a significant reduction in onsite storage requirements for used nuclear fuel.

Several operational challenges would benefit from DOE support, such as digital instrumentation and control. While some of these challenges must be resolved by industry, DOE will likely have a niche role in others. The operational issue that most requires federal assistance is developing advanced safeguards and security technology that can be effectively applied to commercial nuclear power plants. An optimized combination of guns, guards, gates, and advanced detection and response technologies would not only reduce operating costs, but would help provide public confidence in the protection of nuclear power plants.

\section{Facility Options:}

The types of facilities needed to support the operating fleet of LWRs (see Table 2-1) are hot cells capable of handling irradiated fuels and materials, a thermal irradiation capability, materials science laboratories capable of handling both cold and hot materials, and safeguards technology laboratories. Currently, DOE owns and operates such facilities, and they are expected to be maintained for research programs. Other types of capability requirements will likely be identified as this program matures. 
At this time, there is insufficient program definition to make an informed decision on required facility capacity, intensity of use, and possible interference of LWR support programs with other R\&D programs. DOE will collect and analyze requirements as the program matures.

Table 2-1. Facilities considered for support of light water reactor research and development.

\begin{tabular}{|l|l|l|}
\hline Facility & Location & Function \\
\hline Advanced Test Reactor & Idaho National Laboratory & Advanced fuels and materials irradiation \\
\hline High Flux Isotope Reactor & Oak Ridge National Laboratory & Advanced fuels and materials irradiation \\
\hline Annular Core Research Reactor & Sandia National Laboratory & Transient power testing of fuel samples \\
\hline Transient Reactor Test Facility & Idaho National Laboratory & $\begin{array}{l}\text { Transient power testing of fuel pins and } \\
\text { bundles }\end{array}$ \\
\hline Hot Fuel Examination Facility & Idaho National Laboratory & $\begin{array}{l}\text { Post-irradiation examination of advanced } \\
\text { fuels and materials }\end{array}$ \\
\hline Irradiated Fuels Examination Laboratory & Oak Ridge National Laboratory & $\begin{array}{l}\text { Post-irradiation examination of advanced } \\
\text { fuels and materials }\end{array}$ \\
\hline $\begin{array}{l}\text { Irradiated Materials Examination and Testing } \\
\text { Facility }\end{array}$ & Oak Ridge National Laboratory & $\begin{array}{l}\text { Examine and test materials irradiated in } \\
\text { light water reactors and the high flux } \\
\text { isotope reactor }\end{array}$ \\
\hline $\begin{array}{l}\text { Materials and Fuels Complex Analytical } \\
\text { Laboratory }\end{array}$ & Idaho National Laboratory & $\begin{array}{l}\text { Support examination of fuels and materials } \\
\text { in to Hot Fuel Examination Facility }\end{array}$ \\
\hline
\end{tabular}

\section{Summary of Reviewer Comments}

1. Expand discussion of operation beyond 60 years to include a more complete review of the needs for ensuring long term safe operations.

2. Add more facilities: SNRL Shielded Cells; ANL B212, B206, B315; ORELA; LANSCE; RPL 325; HFIR.

\subsection{Workforce Development}

Although workforce development is a major issue for both the nuclear industry and federal government agencies, DOE fills an important niche in developing the workforce of tomorrow. In particular, nuclear engineers, chemists and chemical engineers with advanced degrees fill positions in national laboratories, federal agencies, universities, and the medical profession, far more so than in the nuclear industry. DOE's support of nuclear engineering education programs, including university reactors and other nuclear infrastructure, is crucial to supplying the next generation of research personnel and university faculty. 
Relative to other countries that have surpassed the United States in several areas of nuclear technology development, United States universities are still held in the highest regard throughout the world. Maintaining a strong nuclear engineering education program is a key asset in providing United States leadership in global nuclear energy.

Future needs for educated and trained individuals in the general areas of nuclear science and engineering has

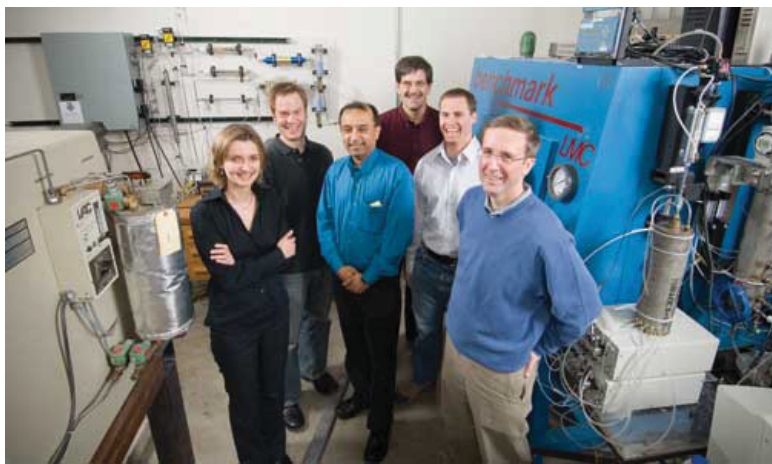

Figure 2-3. The University of Wisconsin is one of two dozen major universities that are turning out the future nuclear research and development workforce. been well documented for more than a decade by the American Nuclear Society, the American Society for Engineering Education, the Health Physics Society, the National Academies of Science and Engineering, the American Physical Society, and the Nuclear Energy Institute. The nuclear industry has predicted a significant shortfall in trained operations staff, design and development engineers, research scientists and engineers, and building and operational tradecraft and construction workers. Additional concerns include imbalances in supply versus demand; levels of undergraduate and graduate student enrollments; the decline in university education programs, research reactors, and other facilities; the image of the discipline, a general lack of excitement; and low visibility of the technical field. Aggressive steps need to be taken immediately and sustained for the long-run to ensure availability of a viable nuclear workforce.

The following types of research and education facilities are available at multiple universities:

- Nuclear reactors and accelerators for research and training

- $\quad$ Radiation measurement laboratories

- $\quad$ Computational facilities

- Instrumentation and control laboratories

- Heat transfer and fluid transport measurement laboratories

- $\quad$ Radiochemistry laboratories

- $\quad$ Hot cells and radioactive material handling capabilities

- $\quad$ Radiological environment training facilities

- $\quad$ Maintenance and repair training facilities 


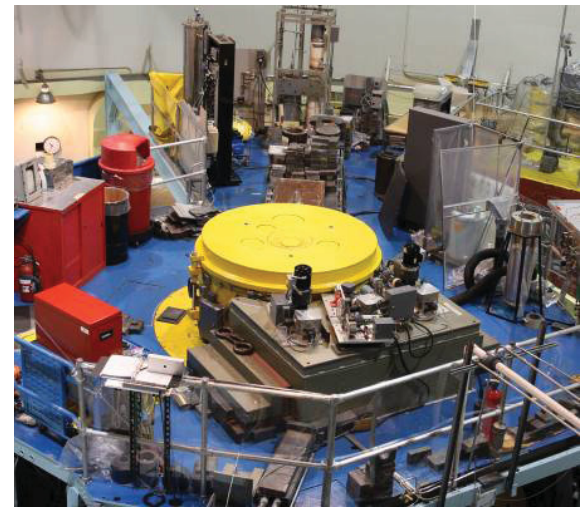

Figure 2-4. The MIT reactor is involved in Department of Energy research and development programs.

\section{Facility Options:}

In the past year, DOE has revamped its educational support program in the light of recommendations from key stakeholders. DOE should continue stewardship support of nuclear engineering education programs while establishing confidence in students about its stability. It is particularly important to ensure that existing university nuclear infrastructure remains intact and safely functioning. To supplement campus capabilities, expanded use of DOE assets such as the Advanced Test Reactor National Scientific User Facility should be encouraged. Additional user facilities, such as hot cells, should be established.

\section{Summary of Reviewer Comments}

1. This section is out of place; should be moved up to the front of the report

2. The National Science Foundation should take over responsibility for nuclear engineering education rather than leaving it with the Department of Energy. 


\subsection{Sustainable Fuel Cycle}

DOE currently has significant activities that target a sustainable fuel cycle through its Advanced Fuel Cycle Initiative. The major R\&D categories are irradiated fuel separations and advanced fuel development.

Ideally, the required capabilities would be consolidated into a new multipurpose facility that has been called AFCF. This proposal is still being studied to determine whether it will be a new facility, or modified existing structures, or a set of capabilities that are geographically separated.

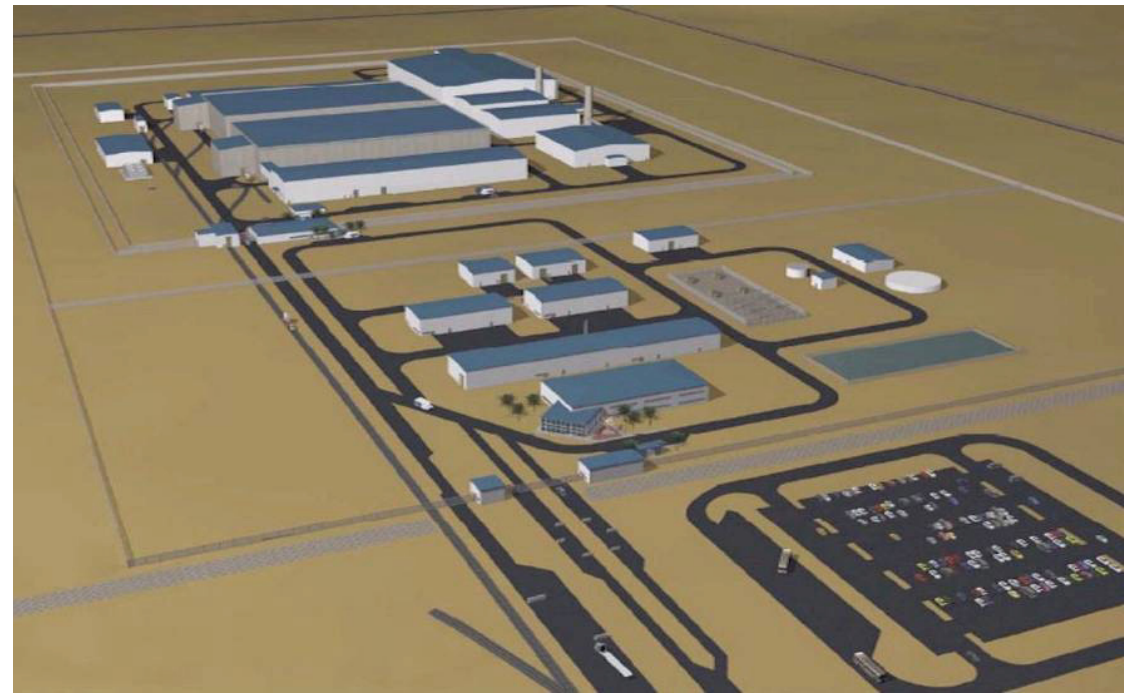

Figure 2-6. Artist's conception of a new consolidated Advanced Fuel Cycle Facility. The Advanced Fuel Cycle Facility's capabilities may actually be distributed among upgraded existing facilities.

\section{Facility Options:}

The proposed AFCF

represents the scale of commitment necessary to develop a sustainable fuel cycle, housing all the radiochemistry, hot cell, and glove box capabilities needed for the required R\&D. As a practical matter, funding for timely completion of a green field facility may not be available. DOE is assessing existing structures for potential modification with some additional construction as a lower cost AFCF possibility. In evaluating this brown field option, one consideration is the capital cost required for the entire supporting infrastructure that must be in place for a major nuclear facility like $\mathrm{AFCF}$. If the capabilities of AFCF are geographically distributed, the operational cost of transportation and multiple highly secure sites must be included in the life-cycle cost.

Significant advantages to building a new facility include the ability to integrate safety, security, and safeguards, to demonstrate modular construction for a fuel cycle facility, and to consolidate operations at a single secure site. DOE could consider phasing the design of the facility so that it is done after the first wave of new LWR modular plant designs. By using the same team of commercial designers, there could be a significant cost savings and efficiency.

Until the AFCF is ready, DOE will need to upgrade and maintain existing facilities for R\&D with modest amounts of materials. As a bridging measure, DOE also could investigate the potential for using the world-class facilities available through our international collaborators. This option seems especially attractive with France and Japan, where the United States already has strong agreements in place.

Table 2-2 lists facilities that DOE has evaluated for possible use in assembling a brown field version of AFCF. This list is being refined to a manageable few options. These facilities are typically at least 40 years old. Safety questions about adaptation to new missions have not been fully evaluated. Safeguards and security issues are only evaluated for facilities inside a currently highly protected area. 
Table 2-2. Existing facilities evaluated by the Department of Energy for support of major Advanced Fuel Cycle Facility functions.

\begin{tabular}{|c|c|c|}
\hline Facility & Location & Function \\
\hline Shielded Cell Facility & $\begin{array}{l}\text { Savannah River National } \\
\text { Laboratory }\end{array}$ & Limited-scale separations \\
\hline Engineering Development Laboratory & $\begin{array}{l}\text { Savannah River National } \\
\text { Laboratory }\end{array}$ & Process Development \\
\hline H-Canyon/F-Canyon & Savannah River Site & Production scale separations \\
\hline Receiving Basin for Offsite Fuels & Savannah River Site & Fuel storage \\
\hline Irradiated Fuel Examination Facility & $\begin{array}{l}\text { Oak Ridge National } \\
\text { Laboratory }\end{array}$ & $\begin{array}{l}\text { Examination and head-end separations } \\
\text { process }\end{array}$ \\
\hline Cold Test Facility & $\begin{array}{l}\text { Oak Ridge National } \\
\text { Laboratory }\end{array}$ & Process development \\
\hline $\begin{array}{l}\text { Radiochemical Engineering Development Center } \\
\text { (REDC-7920) }\end{array}$ & $\begin{array}{l}\text { Oak Ridge National } \\
\text { Laboratory }\end{array}$ & Separations \\
\hline $\begin{array}{l}\text { Radiochemical Engineering Development Center } \\
\text { (REDC-7930) }\end{array}$ & $\begin{array}{l}\text { Oak Ridge National } \\
\text { Laboratory }\end{array}$ & Separations \\
\hline Chemical and Materials Research & $\begin{array}{l}\text { Los Alamos National } \\
\text { Laboratory }\end{array}$ & Separations \\
\hline PF-4 at TA-55 & $\begin{array}{l}\text { Los Alamos National } \\
\text { Laboratory }\end{array}$ & Oxide fuel fabrication \\
\hline Engine Maintenance and Disassembly & Nevada Test Site & Separations \\
\hline Pluto Disassembly Facility & Nevada Test Site & Separations \\
\hline Fuels and Materials Examination Facility & $\begin{array}{l}\text { Pacific Northwest National } \\
\text { Laboratory }\end{array}$ & Separations and fuel development \\
\hline Radiochemical Processing Laboratory & $\begin{array}{l}\text { Pacific Northwest National } \\
\text { Laboratory }\end{array}$ & Separations \\
\hline Fluorinel Dissolution and Storage Facility, CPP-666 & Idaho National Laboratory & Pilot-scale separations \\
\hline Fuel Processing Facility, CPP-691 & Idaho National Laboratory & Pilot-scale separations \\
\hline New Waste Calcine Facility, CPP-659 & Idaho National Laboratory & Pilot-scale separations \\
\hline Fuel Manufacturing Facility, MFC-704 & Idaho National Laboratory & Fuel development \\
\hline Hot Fuel Examination Facility, MFC-785 & Idaho National Laboratory & Separations \\
\hline Fuel Conditioning Facility, MFC-765 & Idaho National Laboratory & $\begin{array}{l}\text { Engineering-scale electrochemical } \\
\text { separations }\end{array}$ \\
\hline Remote Analytical Laboratory, CPP-684 & Idaho National Laboratory & Separations \\
\hline MFC Analytical Laboratory & Idaho National Laboratory & Process development \\
\hline
\end{tabular}




\section{Summary of Reviewer Comments}

1. Add more facilities: SRNL Actinide Science \& Separations Development Labs; ANL B205, B212, B206, B308, B370, B315

2. The current AFCI program includes more elements than the separations and fuel development categories described in this section.

3. Report does not make a clear case for a combined facility.

4. NRC needs additional validation data for higher fuel enrichments and manufacturing safety, which would require criticality facilities.

5. The report should mention the need for facilities that can obtain validation data for burnup credits for storage and transportation of irradiated fuel.

\subsubsection{Irradiated Fuel Separations}

Irradiated fuel separations include the disassembly and dissolution of irradiated nuclear fuel; recovery of plutonium, uranium, and minor actinides; and fabrication of robust waste forms. Facilities needed to fill this requirement form a subset of the AFCF set of facilities.

\section{Facility Options:}

The R\&D separations campaign has a structured development plan that calls for near-term use of existing actinide chemistry laboratories, shielded hot cells, process equipment development laboratories, and waste form laboratories at the national laboratories and universities. Engineering-scale testing will require an AFCF. Use of the Japanese, French, or British facilities could be considered as a bridging measure. Each country has capacity for engineering-scale development.

\subsubsection{Advanced Fuel Development}

Advanced fuel development will include ultra long-lived, reliable LWR fuel and fast reactor and high-temperature reactor fuel. Currently, advanced LWR fuel is developed by the commercial industry; however, break through, higher-risk technology, including mixed-oxide fuels, will require federal participation in the development. In general, development facilities for fast reactor fuel will be adequate for advanced LWR fuel (see Table 2-3). Facility requirements include the following:

- $\quad$ Fuel development laboratories using simulates and surrogates

- Material science laboratories for cladding development

- $\quad$ Fuel development laboratories with actinide confinement, including shielded glove boxes

- Shielded hot cells for remote fabrication of rodlets and pins and for post-irradiation examination

- $\quad$ Thermal and fast accelerated irradiation capability for pins and rodlets 
- Irradiation capability for full fuel assemblies or full fuel rods

- $\quad$ Transient testing to fuel failure capability.

\section{Facility Options:}

Fuel development is a longstanding activity for DOE. New aspects of these activities are actinide bearing fuel for fast reactors and particle fuel for high-temperature reactors. All requirements could be met by AFCF when it is completed.

DOE currently has operating facilities that support advanced fuel development. Some investment in new equipment and other capabilities is required. As a bridging measure, fabricating and irradiating advanced nuclear fuel in international facilities might be possible as a part of an international collaboration. The Transient Reactor Test Facility (TREAT) should be restarted as it represents an essential testing capability not available in France, Japan, or the United Kingdom.

Table 2-3. Existing facilities considered for support of advanced fuel development.

\begin{tabular}{|c|c|c|}
\hline Facility & Location & Function \\
\hline Shielded Cell Facility & $\begin{array}{l}\text { Savannah River National } \\
\text { Laboratory }\end{array}$ & Limited scale tests \\
\hline Irradiated Fuel Examination Facility & $\begin{array}{l}\text { Oak Ridge National } \\
\text { Laboratory }\end{array}$ & Post-irradiation examination \\
\hline Cold Test Facility & $\begin{array}{l}\text { Oak Ridge National } \\
\text { Laboratory }\end{array}$ & Process development \\
\hline Chemical and Materials Research & $\begin{array}{l}\text { Los Alamos National } \\
\text { Laboratory }\end{array}$ & Remote fabrication \\
\hline PF-4 at TA-55 & $\begin{array}{l}\text { Los Alamos National } \\
\text { Laboratory }\end{array}$ & Oxide fuel fabrication \\
\hline Fuels and Materials Examination Facility & $\begin{array}{l}\text { Pacific Northwest National } \\
\text { Laboratory }\end{array}$ & Subassembly scale fabrication \\
\hline Radiochemical Processing Laboratory & $\begin{array}{l}\text { Pacific Northwest National } \\
\text { Laboratory }\end{array}$ & Process development \\
\hline Fuel Manufacturing Facility, MFC-704 & Idaho National Laboratory & Multiscale fuel development \\
\hline Hot Fuel Examination Facility, MFC-785 & Idaho National Laboratory & Post-irradiation examination \\
\hline Fuel Conditioning Facility, MFC-765 & Idaho National Laboratory & Remote fuel fabrication \\
\hline Remote Analytical Laboratory, CPP-684 & Idaho National Laboratory & Shielded remote fabrication \\
\hline Materials and Fuels Complex Analytical Laboratory & Idaho National Laboratory & Process development \\
\hline B\&W Fuel Development Laboratories & B\&W, Lynchburg, VA & Particle fuel fabrication \\
\hline High Flux Isotope Reactor & $\begin{array}{l}\text { Oak Ridge National } \\
\text { Laboratory }\end{array}$ & Thermal and shielded fast irradiation \\
\hline Advanced Test Reactor & Idaho National Laboratory & Thermal and shielded fast irradiation \\
\hline LANCE Materials Test Station (proposed) & $\begin{array}{l}\text { Los Alamos National } \\
\text { Laboratory }\end{array}$ & Fast irradiation using accelerator \\
\hline Phenix & CEA, Marcoule, France & Rodlets under fast irradiation until 2009 \\
\hline Joyo & JAEA, Oarai, Japan & Fast irradiation up to subassembly size \\
\hline Monju & JAEA, Tsuruga, Japan & Fast irradiation up to partial core loading \\
\hline BOR-60 & Russia & Fast irradiation of rodlets or pins \\
\hline Fast Flux Test Facility & Pacific Northwest National & Fast irradiation through subassembly \\
\hline
\end{tabular}


Table 2-3. (continued).

\begin{tabular}{|l|l|l|}
\hline Facility & Location & Function \\
\hline Annular Core Research Reactor & Laboratory & size \\
\hline Transient Reactor Test Facility & $\begin{array}{l}\text { Sandia National } \\
\text { Laboratory }\end{array}$ & Transient testing of fuel samples \\
\hline High-Temperature Engineering Test Reactor & Idaho National Laboratory & Transient testing of fuel pin bundles \\
\hline
\end{tabular}

\section{Summary of Reviewer Comments}

1. Should make it clear that the risks described are development risks, not technology risks.

2. Add more facilities: SRNL Reactor Fuel Fabrication Laboratory; ANL B212 and B205; ORNL TRISO Lab, IFEL, ORELA; LANSCE

3. ACRR can test small fuel pin bundles in addition to samples

\subsection{Next Generation Reactors}

The United States is a founding member of the Generation-IV International Forum. The Generation-IV International Forum members have agreed to collaborate on six different types of next generation reactor systems:

- $\quad$ Very High-Temperature Reactor (VHTR)

- $\quad$ Supercritical Water Reactor (SCWR)

- $\quad$ Gas-Cooled Fast Reactor (GFR)

- $\quad$ Lead-Cooled Fast Reactor (LFR)

- $\quad$ Sodium-Cooled Fast Reactor (SFR)

- $\quad$ Molten Salt Reactor (MSR)

Each member of the Generation-IV International Forum is free to focus on whatever systems it chooses. The United States has elected to focus on the VHTR and the SFR. VHTR R\&D is centered on the Next Generation Nuclear Plant (NGNP) project. The United States' activities for the other four systems are minimal. 


\section{Summary of Reviewer Comments}

1. Advanced sensor technology development is needed.

2. Nuclear data facilities should be identified as needed

\subsubsection{High-Temperature Reactor}

The high-temperature reactor is a class of reactors, including the VHTR, with potential for industrial heat applications at $700^{\circ} \mathrm{C}$ and above. DOE currently has a robust NGNP program that is in the process of determining design parameters for the first prototype HTR while developing fuels materials and components for the prototype that is to be completed in approximately the 2021 timeframe.

Facility Options:

The NGNP prototype requires design and construction. A new Components Test Facility for heat transport/exchange, electrical power conversion, and instrumentation and control, is needed in support of the NGNP project. The Nuclear Hydrogen Initiative will likely require a new engineering development facility to move to engineering-scale production in order to stay on track with NGNP.

The facilities listed in Table 2-4 should be maintained and upgraded as needed to maintain momentum in the NGNP project. As with its other programs, DOE should consider how international assets can be effectively used. Examples would be the High-Temperature Engineering Test Reactor in Oarai, Japan, and the Helium Component Test Facility at Cadarache, France.

Table 2-4. Existing facilities considered for support of Next Generation Nuclear Plant research and development.

\begin{tabular}{|c|c|c|}
\hline Facility & Location & Function \\
\hline Advanced Test Reactor & Idaho National Laboratory & $\begin{array}{l}\text { High-temperature reactor fuels and } \\
\text { graphite irradiation }\end{array}$ \\
\hline TRISO Fuel Laboratory & $\begin{array}{l}\text { Oak Ridge National } \\
\text { Laboratory }\end{array}$ & Fuel kernel and coating fabrication \\
\hline ZPR Cell 5 & $\begin{array}{l}\text { Argonne National } \\
\text { Laboratory }\end{array}$ & $\begin{array}{l}\text { Safety tests involving graphite dust and } \\
\text { helium/air mixing }\end{array}$ \\
\hline $\begin{array}{l}\text { Natural Convection Shutdown Heat Removal Test } \\
\text { Facility }\end{array}$ & $\begin{array}{l}\text { Argonne National } \\
\text { Laboratory }\end{array}$ & $\begin{array}{l}\text { Benchmark safety experiments for } \\
\text { shutdown heat removal }\end{array}$ \\
\hline Graphite Laboratory & $\begin{array}{l}\text { Oak Ridge National } \\
\text { Laboratory }\end{array}$ & $\begin{array}{l}\text { Safety tests involving graphite dust and } \\
\text { helium/air mixing }\end{array}$ \\
\hline Safety and Tritium Applied Research Laboratory & Idaho National Laboratory & $\begin{array}{l}\text { Tritium transport through alloys at high } \\
\text { temperature }\end{array}$ \\
\hline Hot Fuel Examination Facility & Idaho National Laboratory & $\begin{array}{l}\text { Post-irradiation examination of } \\
\text { high-temperature reactor fuels and } \\
\text { materials; safety and fission product } \\
\text { transport testing }\end{array}$ \\
\hline Irradiated Fuels Examination Laboratory & $\begin{array}{l}\text { Oak Ridge National } \\
\text { Laboratory }\end{array}$ & $\begin{array}{l}\text { Post-irradiation examination of } \\
\text { high-temperature reactor fuels and } \\
\text { materials }\end{array}$ \\
\hline Core Conduction Cool-down Test Facility & $\begin{array}{l}\text { Oak Ridge National } \\
\text { Laboratory }\end{array}$ & $\begin{array}{l}\text { Accident condition testing of irradiated } \\
\text { high-temperature reactor fuels }\end{array}$ \\
\hline Materials and Fuels Complex Analytical Laboratory & Idaho National Laboratory & $\begin{array}{l}\text { Support examination of fuels and } \\
\text { materials in the Hot Fuel Examination }\end{array}$ \\
\hline
\end{tabular}




\begin{tabular}{|l|l|l|}
\hline Facility & Location & Function \\
\hline High-Temperature Engineering Test Reactor & Facility \\
\hline Helium Component Test Facility & Cadarache, France & $\begin{array}{l}\text { Testing of helium handling equipment for } \\
\text { reactor use }\end{array}$ \\
\hline
\end{tabular}

\section{Summary of Reviewer Comments}

1. Add more facilities: PNNL Materials Sciences Laboratory and Physical Sciences Facility; SRNL Hydrogen Processing Technology Laboratories; ANL B212, B206, APS; ORNL TRISO facility and HFIR.

2. National Hydrogen Initiative pilot testing requirements could be coupled with the Components Test Facility to reduce cost and increase benefits.

3. The HTGR research facilities in the Republic of South Africa are not included.

\subsubsection{Sodium Fast Reactor}

Assuming a successful global nuclear renaissance, fast reactors will be commercialized this century in order to ensure a secure, affordable fuel supply and to manage higher actinides that would otherwise be troublesome for waste disposal in deep geologic repositories. Most countries with advanced reactor development programs are developing SFRs. Some of the technology for a sodium-cooled fast reactor is relatively mature. However, R\&D is required for advanced fuel development, safety design, capital cost reduction, and some key components.

Russia has successfully operated the $600-\mathrm{MWe}$ BN-600 for more than 20 years and also operates the BOR-60 test reactor. India is completing construction of a 500-MWe prototype in 2010, with a total of four identical units to be completed at the site on the Bay of Bengal by 2020. China, who is currently building a small fast test reactor, plans rapid deployment of commercial SFR plants during the first half of this century. France, which has operated the Phenix prototype fast reactor and the large 1200-MWe Superphenix plant, plans to construct a new prototype within 15 years. Japan, which currently operates the Joyo test reactor and the Monju demonstration reactor, plans to start up an advanced SFR prototype in 2025. The United States successfully operated the EBR-II for 30 years and the FFTF for 10 years, but has clearly lost its lead in SFR technology. The United States, France, and Japan have recently signed a trilateral agreement for sharing the development of SFR prototypes.

The technology supporting design of SFR systems is extensive, the result of more than 50 years of R\&D in the United States and other countries. However, early technology decisions could significantly impact facility needs and cost. The most fundamental of those decisions is fuel type, either metal or oxide, both having a strong

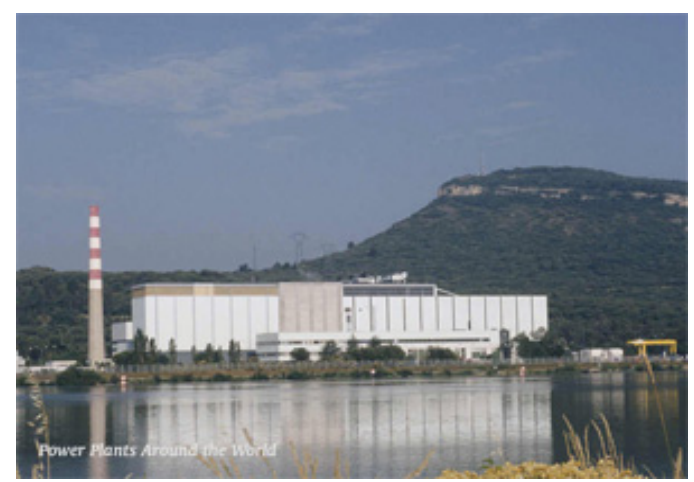

Figure 2-7. The sodium-cooled fast reactor Phenix has served French research and development programs for decades. The Department of Energy currently has advanced fuel experiments under irradiation in Phenix. 
technology base in the United States. Both fuel types require further development to be heavily loaded with minor actinides for transmutation. Metal fuel fabrication and recycling is inherently more compact and can make better use of currently operating facilities. Worldwide experience favors oxide, reflected in the established programs of France, Russia, and Japan. India, China, and South Korea expect to use metal fuel for ultimate commercialization.

At DOE's request, three industry teams are currently developing proposals for a Nuclear Regulatory Commission licensed U.S.-prototype SFR. Some, if not all, of the existing facilities listed in Table 2-5 will be needed to provide support for this project and participation in international SFR collaboration.

Table 2-5. Existing facilities considered for support of sodium-cooled fast reactor research and development.

\begin{tabular}{|c|c|c|}
\hline Facility & Location & Function \\
\hline Advanced Test Reactor & Idaho National Laboratory & Filtered fast irradiation of samples \\
\hline Fuel Conditioning Facility, MFC-765 & Idaho National Laboratory & $\begin{array}{l}\text { Remote fuel fabrication up to } \\
\text { engineering scale }\end{array}$ \\
\hline Fuel Manufacturing Facility, MFC-704 & Idaho National Laboratory & Multiscale fuel development \\
\hline Hot Fuel Examination Facility & Idaho National Laboratory & $\begin{array}{l}\text { Post-irradiation examination of fuels and } \\
\text { materials }\end{array}$ \\
\hline Neutron Radiography Facility & Idaho National Laboratory & $\begin{array}{l}\text { Post-irradiation examination of fuels and } \\
\text { materials }\end{array}$ \\
\hline Materials and Fuels Complex Analytical Laboratory & Idaho National Laboratory & $\begin{array}{l}\text { Support examination of fuels and } \\
\text { materials in the Hot Fuel Examination } \\
\text { Facility }\end{array}$ \\
\hline $\begin{array}{l}\text { Natural Convection Shutdown Heat Removal Test } \\
\text { Facility }\end{array}$ & $\begin{array}{l}\text { Argonne National } \\
\text { Laboratory }\end{array}$ & $\begin{array}{l}\text { Benchmark safety experiments for } \\
\text { shutdown heat removal }\end{array}$ \\
\hline High Flux Isotope Reactor & $\begin{array}{l}\text { Oak Ridge National } \\
\text { Laboratory }\end{array}$ & Filtered fast irradiation of samples \\
\hline Phenix & CEA, Marcoule, France & (2009 shutdown) current irradiation \\
\hline Joyo & Oarai, Japan & Metal and oxide fuel irradiation \\
\hline MONJU & Tsuruga, Japan & Oxide fuel irradiation \\
\hline BOR-60 & Russia & Fast irradiation of rodlets or pins \\
\hline Fast Flux Test Facility & Hanford & $\begin{array}{l}\text { Lead test assembly irradiation and } \\
\text { sodium component testing }\end{array}$ \\
\hline Annular Core Research Reactor & $\begin{array}{l}\text { Sandia National } \\
\text { Laboratory }\end{array}$ & Transient testing of fuel samples \\
\hline Transient Reactor Test Facility & Idaho National Laboratory & Transient testing of fuel pin bundles \\
\hline LANCE Materials Test Station & $\begin{array}{l}\text { Los Alamos National } \\
\text { Laboratory }\end{array}$ & $\begin{array}{l}\text { (Proposed) fast irradiation of using } \\
\text { accelerator }\end{array}$ \\
\hline Chemical and Materials Research & $\begin{array}{l}\text { Los Alamos National } \\
\text { Laboratory }\end{array}$ & Fuel development \\
\hline PF-4 at TA-55 & $\begin{array}{l}\text { Los Alamos National } \\
\text { Laboratory }\end{array}$ & Fuel development; oxide fuel fabrication \\
\hline Irradiated Materials Examination and Testing Facility & $\begin{array}{l}\text { Oak Ridge National } \\
\text { Laboratory }\end{array}$ & $\begin{array}{l}\text { Post-irradiation examination of irradiated } \\
\text { structural materials }\end{array}$ \\
\hline Components and Materials Evaluation Loop & $\begin{array}{l}\text { Argonne National } \\
\text { Laboratory }\end{array}$ & Large sodium component testing \\
\hline Fuels and Applied Science Building & Idaho National Laboratory & $\begin{array}{l}\text { Engineering development laboratory } \\
\text { limited to unenriched uranium use }\end{array}$ \\
\hline Electron Microscopy laboratory & Idaho National Laboratory & Fuel development and post-irradiation \\
\hline
\end{tabular}


Table 2-5. (continued).

\begin{tabular}{|l|l|l|}
\hline Facility & Location & Function \\
\hline $\begin{array}{l}\text { Sodium Plugging Loop for Printed Circuit Heat } \\
\text { Exchangers }\end{array}$ & $\begin{array}{l}\text { Argonne National } \\
\text { Laboratory }\end{array}$ & examination \\
\hline LANSCE-Lujan cross section facilities & $\begin{array}{l}\text { Los Alamos National } \\
\text { Laboratory }\end{array}$ & Experimental nuclear physics \\
\hline Irradiated Fuels Examination Laboratory & $\begin{array}{l}\text { Oak Ridge National } \\
\text { Laboratory }\end{array}$ & $\begin{array}{l}\text { Post-irradiation examination of fuels and } \\
\text { materials }\end{array}$ \\
\hline Sodium Processing Facility & Idaho National Laboratory & $\begin{array}{l}\text { Sodium component testing and } \\
\text { workforce development }\end{array}$ \\
\hline MASURCA & Cadarache, France & Fast critical experiments \\
\hline
\end{tabular}

Facility Options:

A major focus of the SFR research and development program is on advanced materials, component, system, and advanced modeling and simulation to support capital and operating cost reductions while maintaining the inherent safety characteristics of sodium fast reactor technology.. The United States needs an SFR project to drive development and keep pace with the international community.

DOE will need a fast irradiation capability for fuel samples, post-irradiation examination capability, and continued collaboration with international partners for full-size fuel irradiation. DOE also will need a transient test capability that can be shared with the other reactor and fuel development programs.

To accelerate development, DOE could investigate the practicality of collaborating on a single prototype project with France or Japan or both. Japan will have the capability to irradiate both metal and oxide fuel in its Joyo test reactor and Monju will be able to have partial core loadings of advanced actinide-bearing oxide fuel within a few years. INL and the French Commissariat à l'énergie atomique (CEA) have been providing some assistance with the restart of both of those reactors.

Regardless of whether there is collaboration on constructing and operating a prototype reactor, DOE can consider leveraging its current SFR development infrastructure by increased international collaboration. For example, Japan has irradiation capacity, fuel fabrication and recycling capacity, and plans for a sodium technology center. The United States could provide transient testing capability and successful technology experience. INL has lab-to-lab agreements with Japan Atomic Energy Agency (JAEA) centers and could readily coordinate an investigation of the practicality of integrated international programs with Japan, as well as more broadly. At a minimum, the United States can take advantage of the offer by France to take part in the Phenix shutdown tests in 2009 and the offer by Japan to take part in the Monju restart tests from 2008 to 2010. 


\section{Summary of Reviewer Comments}

1. Joyo will not be able to conduct irradiations for four years due to ongoing repairs.

2. Remove inconsistency in treatment of international agreements.

3. Add ANL facilities B308, B206, B212; Hanford FFTF; ORNL LAMDA, IMET, IFEL; SNL SPR CX

4. Should add non-nuclear facilities to study sodium severe accident issues.

\subsubsection{Small Reactor}

The idea of deploying cost-effective smaller nuclear power plants for markets without the extensive infrastructure needed to support a large LWR has been around for a long time. This class of reactors could range from as low as $10 \mathrm{MWe}$ to the 600 -MWe Westinghouse AP-600, which already has Nuclear Regulatory Commission design certification. Worldwide, there are literally dozens of design concepts, some of which are incredibly novel. Although DOE has emphasized downsized LWR technology, small reactor designs often jump ahead to Generation-IV technologies. The most notable example is the Toshiba 4S reactor, which has been proposed as a replacement source of power for diesel generators in the remote town of Galena, Alaska.

Currently, DOE has no requirement to develop a small reactor; therefore, the federal role would be to provide support for licensing new technology that is beyond the normal capability of commercial industry. The first new publicly announced small reactor design, NuScale, has been discussed with the Nuclear Regulatory Commission.

\section{Facility Options:}

Most of the infrastructure capability now anticipated for small reactor development will be available as a result of other reactor development programs. DOE will assess the needs in discussions with established and entrepreneurial businesses that come forward with small reactor designs. There may be a necessary role for government-sponsored R\&D in helping to develop new technologies for this niche market, or alternatively, privately financed government-provided services.

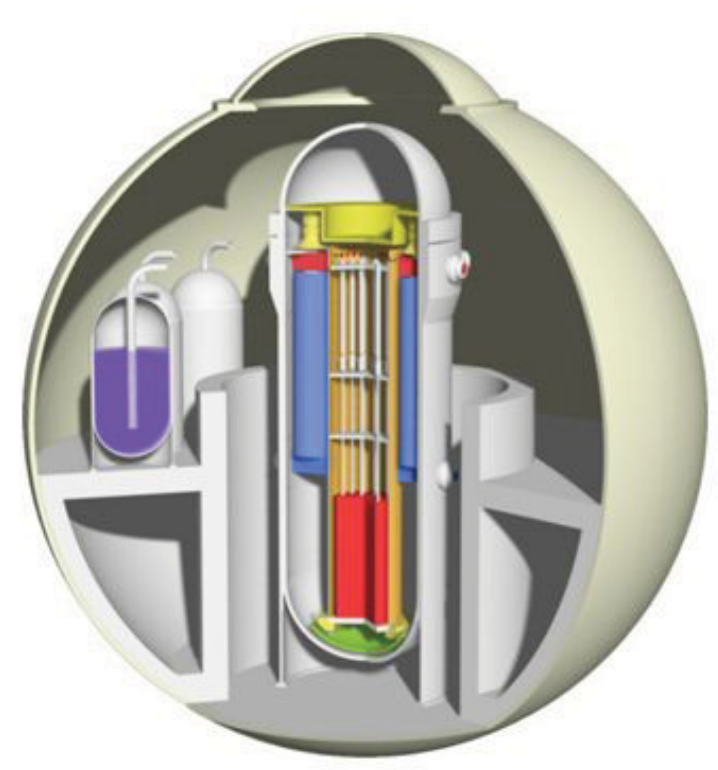

Figure 2-8. The 335-MWe IRIS design by Westinghouse is an example of a small light water reactor that could be used for regional deployment. 


\section{Summary of Reviewer Comments}

1. The list of designs should have included Hyperion and the SMFR.

\subsection{Safeguards and Security}

Robust, innovative safeguards and security systems are highly desirable as deployment of nuclear technology spreads across the globe. Typically, the new, advanced systems are developed under the sponsorship of the National Nuclear Security Administration (NNSA). DOE's facilities that handle, fabricate, convert, or otherwise deal with special nuclear material can serve as test beds for new technology. Much of the testing of advanced safeguards and security systems could be carried out in the proposed AFCF.

\section{Facility Options:}

New nuclear facilities should be designed with safeguards and security included from the initial concept through construction and operation. Any DOE facilities that currently operate with measurable quantities of special nuclear materials can be used as safeguards and security test beds.

\subsection{Modeling and Simulation}

Nuclear engineering is somewhat behind other science and engineering fields in applying advanced computers and software to challenging applications such as aircraft design and simulating supernova explosions. Advanced modeling and simulation aim to employ first principles with extremely high fidelity models to compute near exact solutions to engineering problems. The goal is to accelerate and improve development and understanding of nuclear systems. While modeling and simulation will not replace the need for experiments, they will greatly inform the design of experiments and allow for extrapolation outside the range of experimental data, something that could not be reliably accomplished with past techniques.

Advanced modeling and simulation can become an essential crosscutting technology for the development of next generation reactors, understanding aging issues with currently operating plants, designing improved recycling technologies, and developing long-lived, zero-failure nuclear fuel. Because nuclear systems are very complex (e.g., changing dimensions and transforming materials during extended operation), it is anticipated that the advanced modeling and simulation program will require at least the next generation of computing power.

There are four types of facility requirements for advanced modeling and simulation. First, there is a need for the computing infrastructure, which can be initially shared, but ultimately may need to be dedicated as the program reaches maturity. Second, there will be a need for single-effects test facilities that can perform experiments to validate fundamental computational results. Third, there will be a need for reactors and hot cells to perform complicated integral system tests. Finally, there is a current need to collect, sort, and index legacy and future verification validation data in a knowledge preservation center.

Facility Options: 
Initially, the advanced modeling and simulation program can take advantage of the computing power already available in the Office of Science Laboratories (e.g., Oak Ridge National Laboratory, Pacific Northwest National Laboratory, and Argonne National Laboratory) and the NNSA laboratories (e.g., Sandia, Los Alamos National Laboratory, and Lawrence Livermore National Laboratory). Planning for a dedicated computing facility may be necessary as the program matures (including planning to periodically upgrade the computer systems to prevent obsolescence). The planning should be closely coordinated with NNSA and the Office of Science.

Separate effects test requirements (used to validate portions of simulations) have begun to be identified. To some extent, these tests can be performed in existing facilities or in upgrades to these facilities. Needs for new facilities should be thoroughly documented. Integral testing can rely on reactor fuel development and hot cell facilities, both domestic and international.

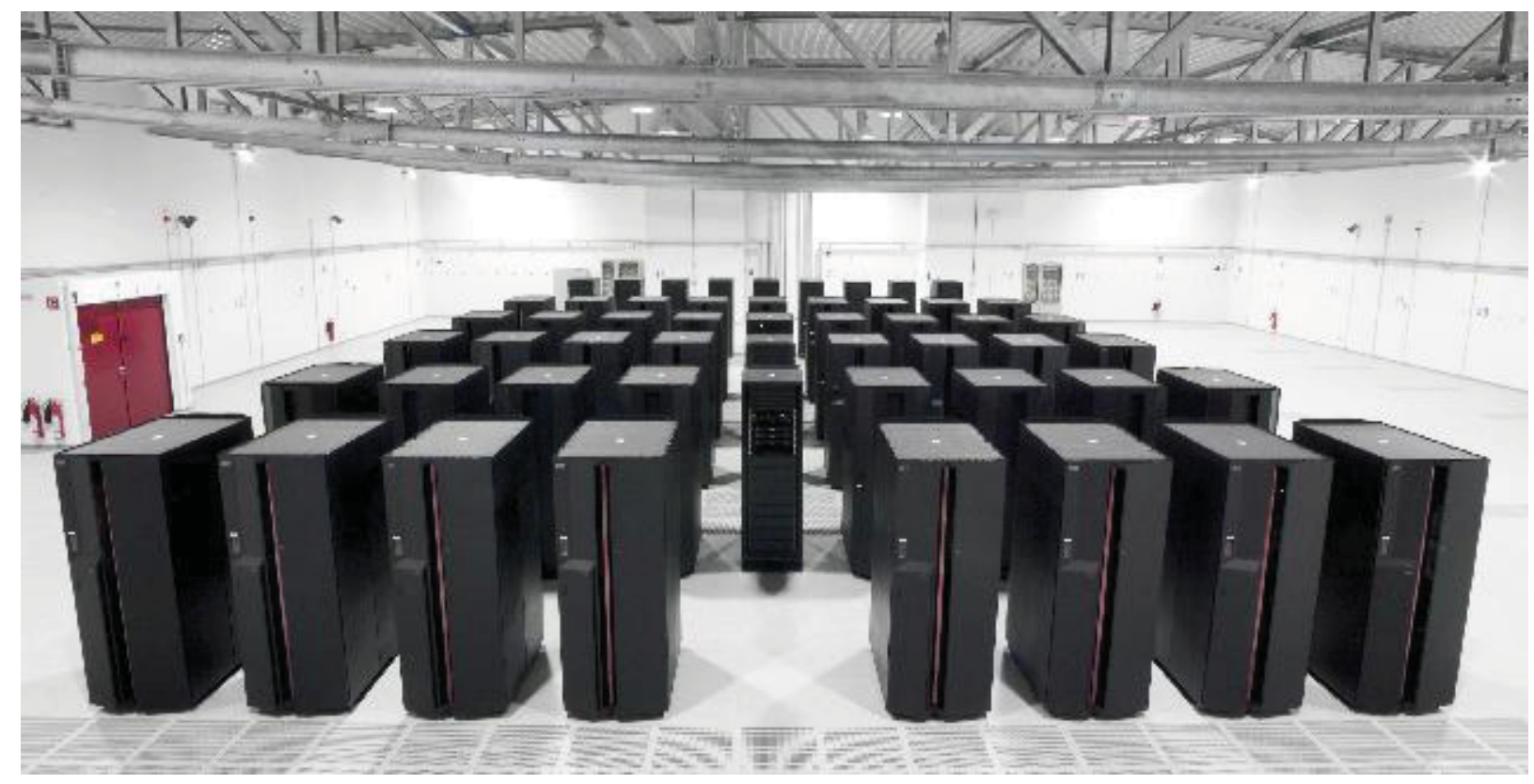

Figure 2-9. Supercomputers have high demand for power, cooling, and cleanliness that requires specialized facilities.

It is critically important that DOE expand its modest effort to archive the legacy data needed to validate advanced modeling and simulation codes. DOE currently owns data from tests that cost billions of dollars that could never be repeated in today's budget and safety environment. Many of the data are in danger of being lost because they are archived on obsolete media, were not preserved when program funding was abruptly terminated, or the knowledgeable researchers are being lost. DOE should immediately start a structured program for archiving physics, fuels, thermal-hydraulics, thermo-physical, safety, and other key data on redundant servers at two or more laboratories. This effort would be in addition to the small programs for knowledge preservation that currently exist with the Nuclear Energy Agency and the somewhat larger knowledge preservation program in the International Atomic Energy Agency.

\section{Summary of Reviewer Comments}

1. PNNL computational capability should be mentioned. 


\subsection{Space and Defense Power Systems}

Plutonium-238 is required for radioisotope power systems used in some National Aeronautics and Space Administration and defense missions. DOE is the sole domestic supplier for this isotope. Although there has been no fresh Pu-238 production in the United States for some 20 years, DOE has been able to procure the material from Russia. However, the Russian supply will be exhausted within a few years. DOE will exhaust its legacy inventory of Pu-238 during the next decade and must decide if, when, and where it will resume domestic production.

\section{$\underline{\text { Facility Options: }}$}

Although DOE-NE has had responsibility for the program for some time, it has never had a full production capability. All of the United States' $\mathrm{Pu}-238$ was produced by Defense Programs as a byproduct of weapons plutonium production using the Savannah River production reactors and reprocessing infrastructure. NE has studied a number of proposals for using the Advanced Test Reactor and the High Flux Isotope Reactor for producing modest quantities of $\mathrm{Pu}-238$. New capability would be required for neptunium target preparation and irradiated target processing. Infrastructure for fabricating the $\mathrm{Pu}-238$ fuel exists at Los Alamos National Laboratory in TA-55. Assembly and testing of radioisotope power systems is done at the Idaho National Laboratory.

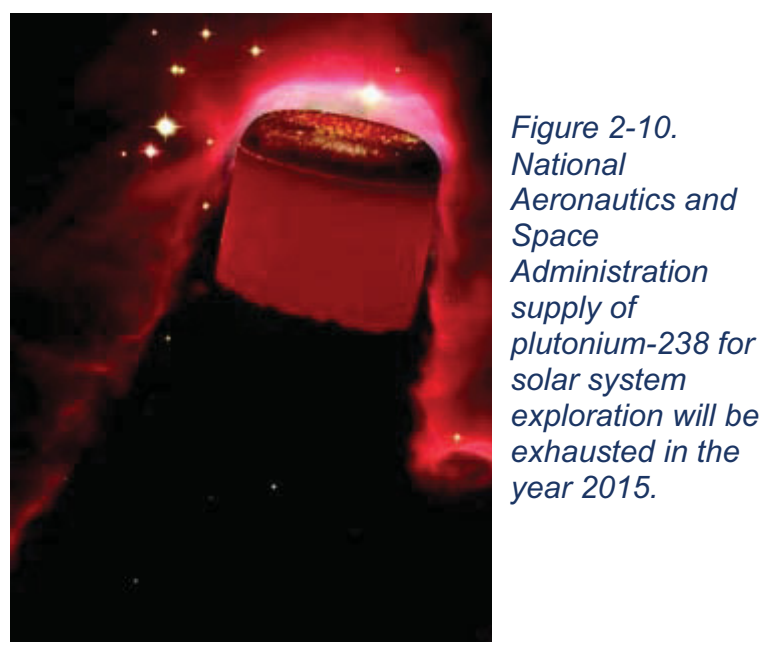

Nuclear power systems for National Aeronautics and Space Administration and other agencies were not identified as an industry priority in the Battelle survey. Nevertheless, they are the responsibility of DOE under the Atomic Energy Act. DOE also is responsible for developing space fission systems, but existing facilities are adequate for the current low level of development activity. Facilities relevant to Pu238 production are listed in Table 2-6. 
Table 2-6. Facilities considered for support of plutonium-238 production, processing, and handling.

\begin{tabular}{|l|l|l|}
\hline Facility & Location & Function \\
\hline Advanced Test Reactor & Idaho National Laboratory & Np target irradiation \\
\hline High Flux Isotope Reactor & $\begin{array}{l}\text { Oak Ridge National } \\
\text { Laboratory }\end{array}$ & Np target irradiation \\
\hline TRIGA reactor(s) (proposed) & TBD & Np target irradiation \\
\hline Fuels and Materials Examination Facility & $\begin{array}{l}\text { Pacific Northwest National } \\
\text { Laboratory }\end{array}$ & Irradiated target processing \\
\hline Fuels Processing Restoration Facility, CPP-691 & Idaho National Laboratory & Irradiated target processing \\
\hline H-Canyon & Savannah River Site & Irradiated target processing \\
\hline $\begin{array}{l}\text { Radiochemical Engineering Development Center } \\
\text { (REDC-7920) }\end{array}$ & $\begin{array}{l}\text { Oak Ridge National } \\
\text { Laboratory }\end{array}$ & Irradiated target separations \\
\hline Remote Analytical Laboratory & Idaho National Laboratory & Potential for multiple functions \\
\hline Fuel Manufacturing Facility & Idaho National Laboratory & Fuel development and target fabrication \\
\hline TA-55/PF-4 & $\begin{array}{l}\text { Los Alamos National } \\
\text { Laboratory }\end{array}$ & Fuel fabrication \\
\hline Space and Security Power Systems Facility & Idaho National Laboratory & Power system assembly and testing \\
\hline Pu-238 Consolidation Facility (proposed) & Idaho National Laboratory & Full Pu-238 fuel cycle capability \\
\hline Zero Power Physics Reactor cell plus upgrades & Idaho National Laboratory & Fuel fabrication \\
\hline TBD facilities & Nevada Test Site & Fuel Fabrication \\
\hline
\end{tabular}

\section{Summary of Reviewer Comments}

1. ORNL REDC Bldg. 7930 should be included.

2. The section does not mention all options considered in the Pu-238 PEIS.

3. The section does not cover specific facilities for space reactor development. 


\section{DOMESTIC AND INTERNATIONAL NUCLEAR R\&D ASSETS}

In the recently prepared report, Nuclear Energy for the Future: Required R\&D Capabilities-An Industry Perspective, Battelle describes 11 different types of facilities (based on facility capabilities). These 11 types of facilities are considered essential to meet the requirements of a viable United States nuclear energy R\&D program over the next 20 to 50 years. The capability categories are as follows:

- Nuclear Education Facilities

- Thermal Irradiation Capability

- $\quad$ Fast Irradiation Capability

- $\quad$ Radiochemistry Laboratories

- Hot Cells for Separations

- Hot Cells for Post-Irradiation Examination

- Thermal Transport

- $\quad$ Fuel Development Laboratories

- $\quad$ Prototype High-Temperature Reactor

- $\quad$ Prototype Fast Reactor

- $\quad$ Specialized Engineering Development Laboratories.

\section{Summary of Reviewer Comments}

1. Add or expand information on the following UK facilities: British Technology Centre, Windscale Laboratory High Activity Shielded Hot Cells, the UK Preston fuel development and engineering facilities.

2. Safety testing should be included.

3. Add the SNL SPRF critical assembly capability; expand ACRR capability to include education.

\subsection{Facility Capabilities}

Table 3.1 lists the major domestic (i.e., DOE, commercial, and university) and international facilities that have been considered in this study and maps them with the applicable capability type from the list above. As noted, several of the facilities currently support or could readily support more than one of the capability categories. Facility descriptions are provided in Sections 3.3 and 3.4 for the key facilities that are included in the evaluation matrices (see Section 3.2) and for selected major international facilities, respectively. As needed, additional facility descriptions will be included in later revisions of this document. 
Table 3-1. Facility/capability matrix table.

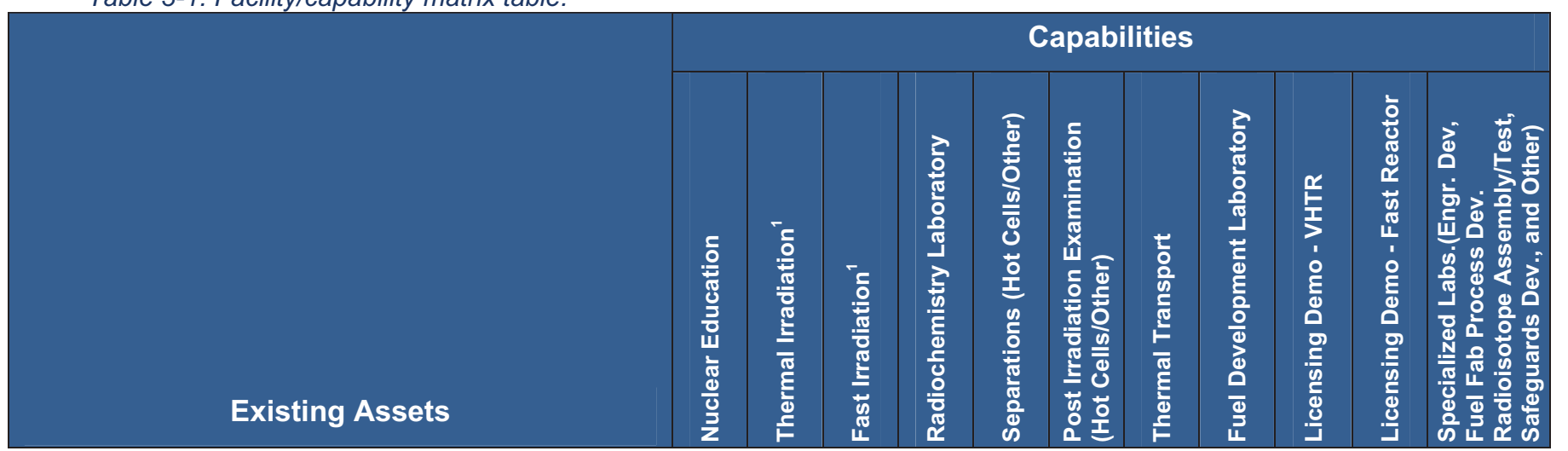

U.S. DOE National Laboratories

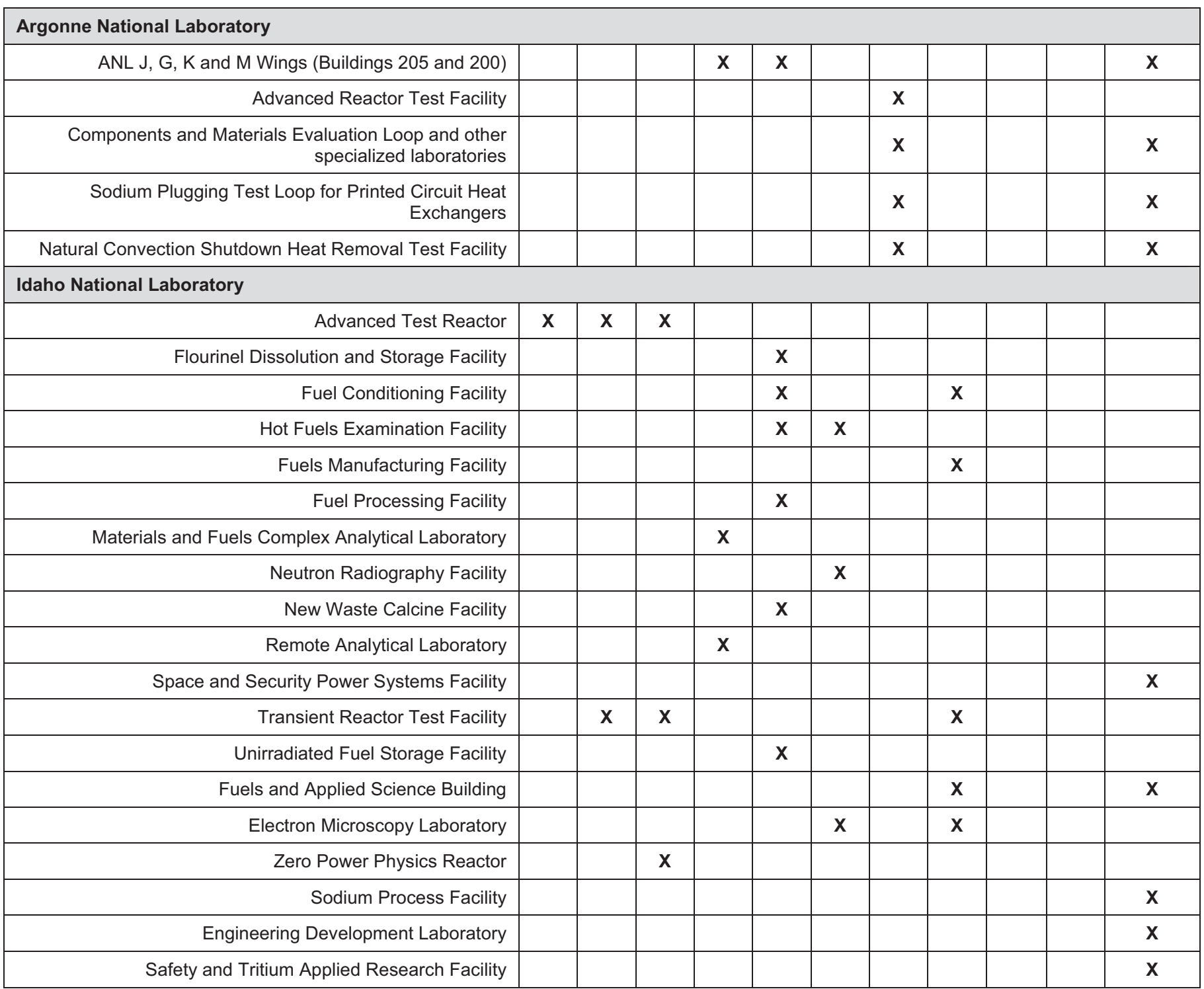


Table 3-1. (continued).

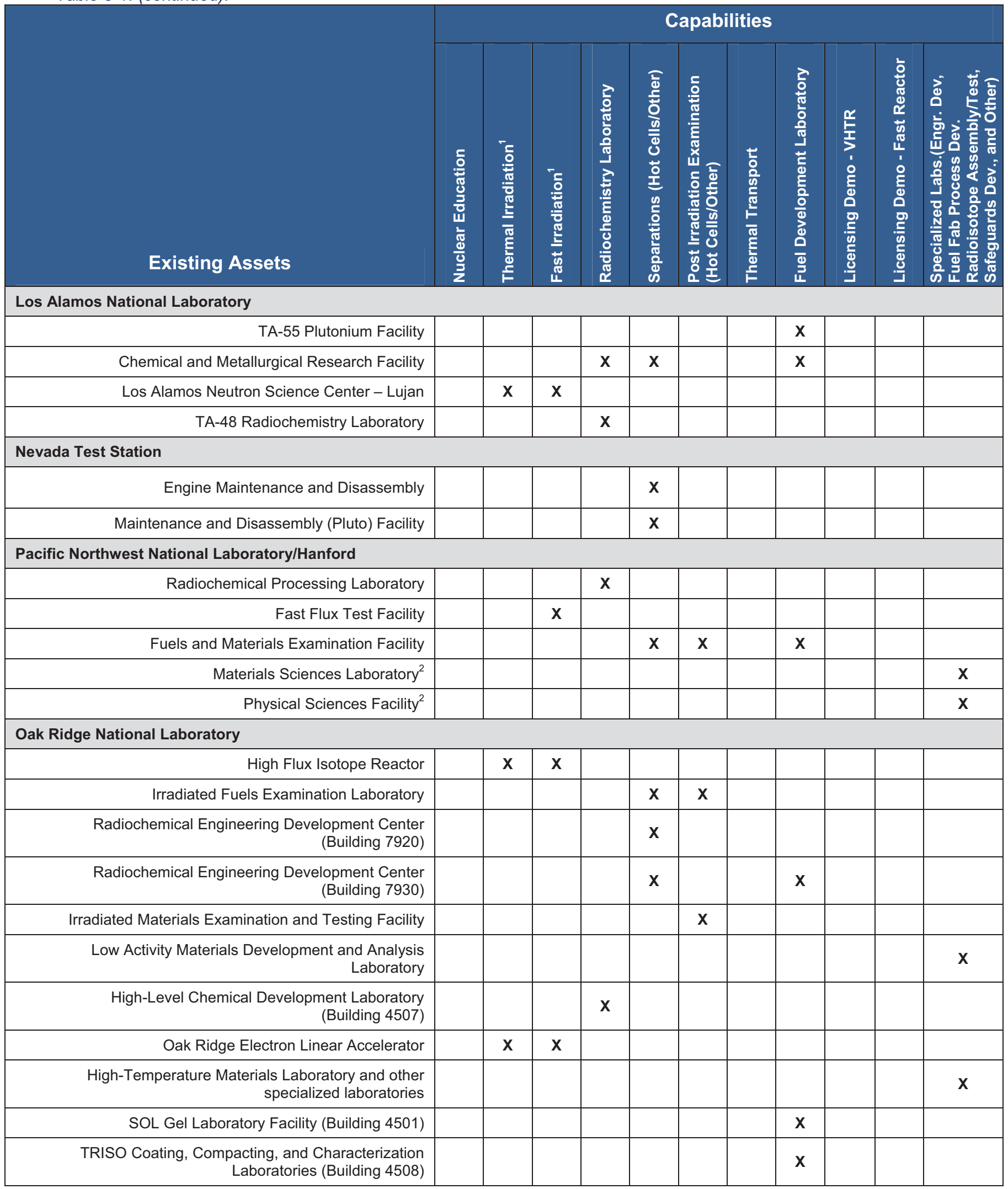


Table 3-1. (continued).

\begin{tabular}{|c|c|c|c|c|c|c|c|c|c|c|c|}
\hline & \multicolumn{11}{|c|}{ Capabilities } \\
\hline Existing Assets & 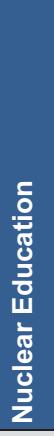 & 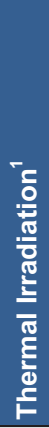 & 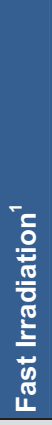 & 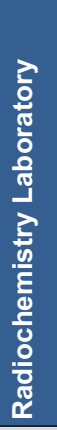 & 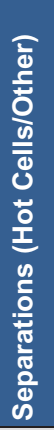 & 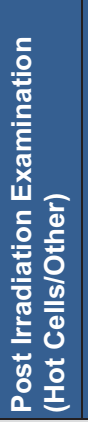 & 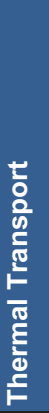 & 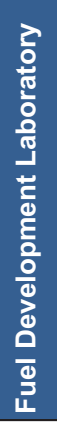 & 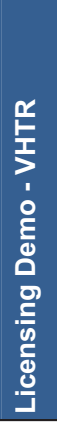 & 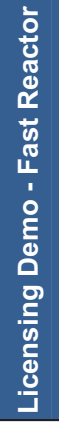 & 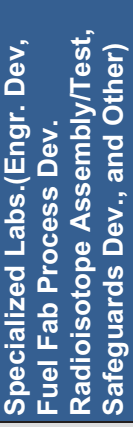 \\
\hline \multicolumn{12}{|l|}{ Sandia National Laboratory } \\
\hline Shielded Cell Facility - High-Level Cells & & & & & $\mathbf{x}$ & & & $\mathbf{x}$ & & & \\
\hline Receiving Basin for Offsite Fuel & & & & & $\mathbf{x}$ & & & & & & \\
\hline Engineering Development Laboratory & & & & & & & & & & & $\mathbf{x}$ \\
\hline H-Canyon & & & & & $\mathbf{x}$ & & & & & & \\
\hline
\end{tabular}

U.S. Commercial

\begin{tabular}{|c|c|c|c|c|}
\hline \multicolumn{5}{|l|}{ General Electric } \\
\hline Vallecitos Nuclear Center & $\mathbf{x}$ & $\mathbf{x}$ & & \\
\hline \multicolumn{5}{|l|}{ Westinghouse } \\
\hline Fuel Manufacturing Plant, Columbia S.C. & & & $\mathbf{X}$ & \\
\hline Oxide Fuels Complex, Springfields, United Kingdom & & & $\mathbf{x}$ & $\mathbf{x}$ \\
\hline Hot Cell Facility & & $\mathbf{x}$ & & \\
\hline R\&D Laboratories & & & & $\mathbf{x}$ \\
\hline B\&W Fuel Fabrication Facility & & & $\mathbf{X}$ & \\
\hline
\end{tabular}

\section{U.S. University}

\section{Massachusetts Institute of Technology}

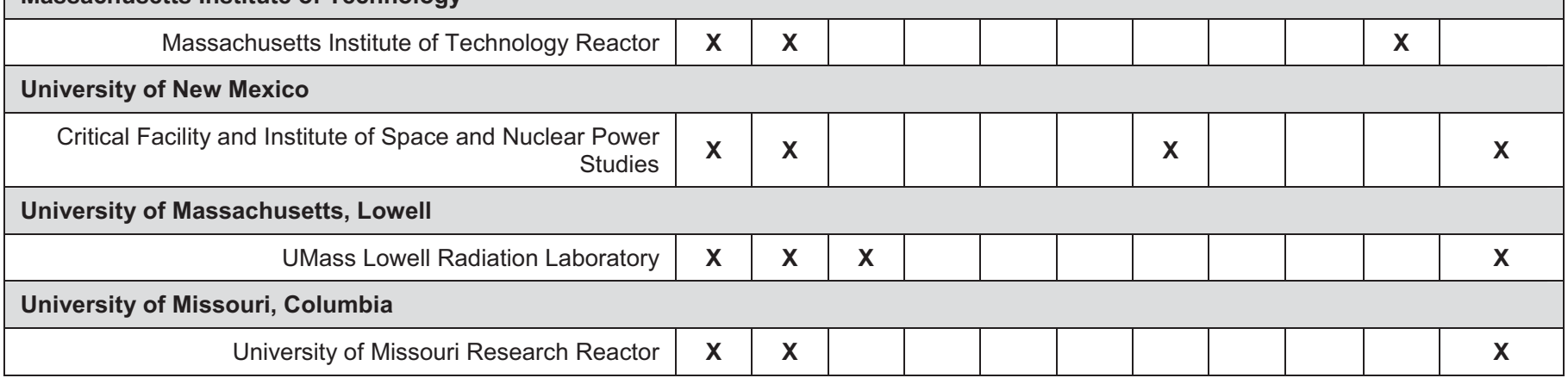


Table 3-1. (continued).

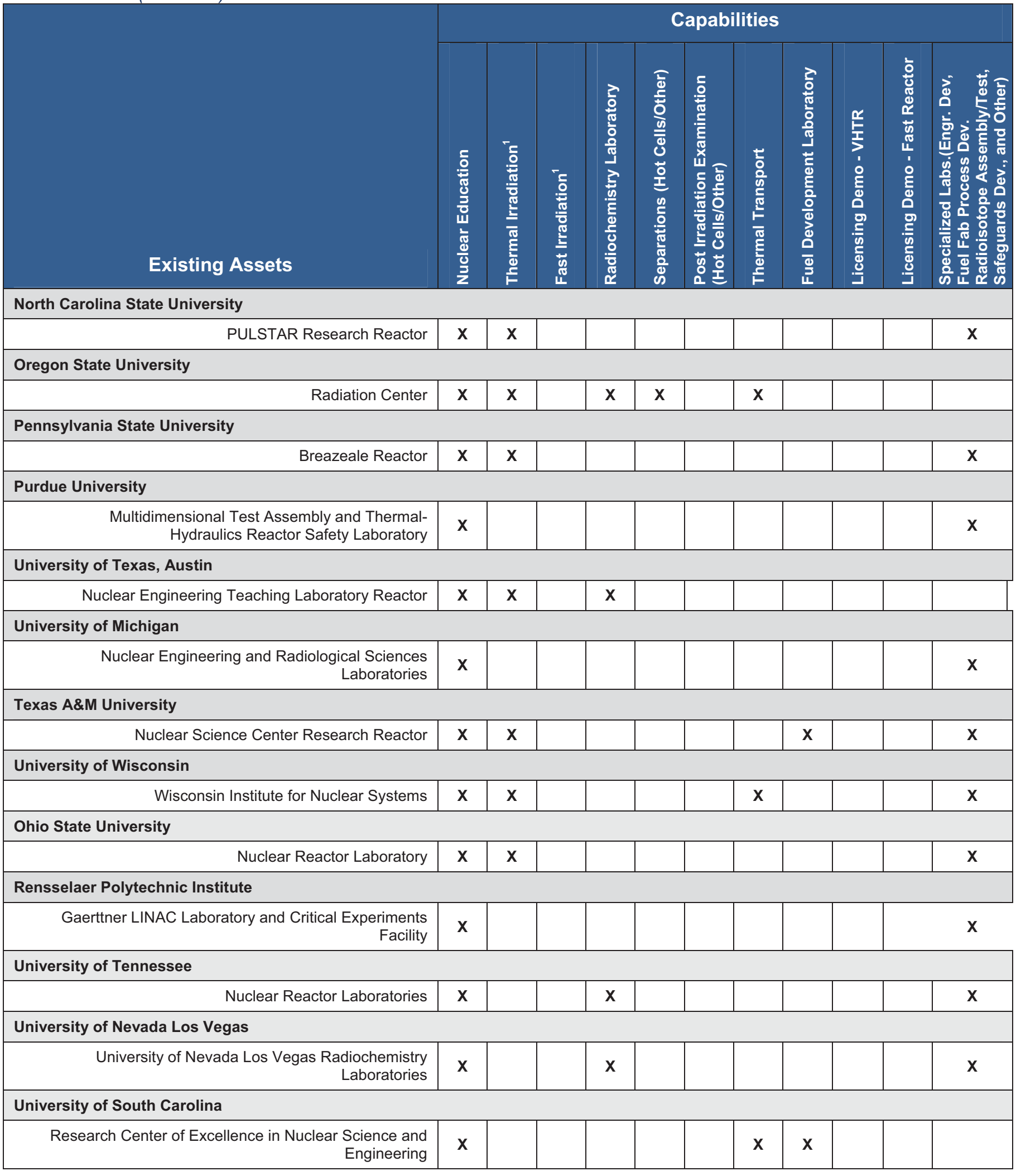


Table 3-1. (continued).

\begin{tabular}{|c|c|c|c|c|c|c|c|c|c|c|c|}
\hline & \multicolumn{11}{|c|}{ Capabilities } \\
\hline Existing Assets & 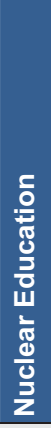 & 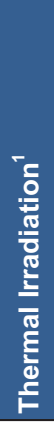 & 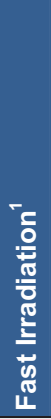 & 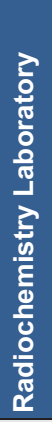 & 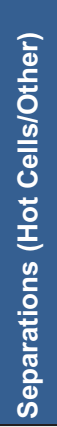 & 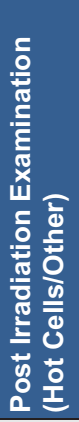 & 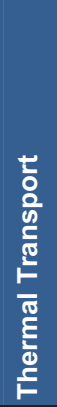 & 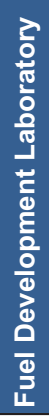 & 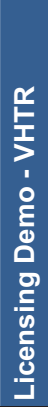 & 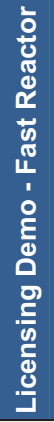 & 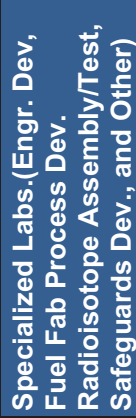 \\
\hline \multicolumn{12}{|l|}{ Boise State University } \\
\hline Nuclear Engineering Materials and Separations Laboratory & $\mathbf{x}$ & & & & $\mathbf{x}$ & & & & & & $\mathbf{x}$ \\
\hline \multicolumn{12}{|l|}{ Georgia Tech } \\
\hline Nuclear and Radiological Engineering Facilities & $\mathbf{x}$ & & & & & & & & & & $\mathbf{x}$ \\
\hline \multicolumn{12}{|l|}{ Idaho State University } \\
\hline Accelerator Center and Nuclear Engineering Laboratory & $\mathbf{x}$ & & & & & & & & & & $\mathbf{x}$ \\
\hline \multicolumn{12}{|l|}{ University of Florida } \\
\hline $\begin{array}{r}\text { University of Florida Training Reactor and Innovative } \\
\text { Nuclear Space Power and Propulsion Institute }\end{array}$ & $\mathbf{x}$ & $\mathbf{x}$ & & & & $\mathbf{x}$ & $\mathbf{x}$ & & $\mathbf{x}$ & & $\mathbf{x}$ \\
\hline $\begin{array}{r}\text { University of California, Berkley Nuclear Engineering } \\
\text { Laboratories }\end{array}$ & $\mathbf{x}$ & & & & & & $\mathbf{x}$ & & & & $\mathbf{x}$ \\
\hline
\end{tabular}

\section{International}

\begin{tabular}{|c|c|c|c|c|c|c|c|c|}
\hline \multicolumn{9}{|l|}{ United Kingdom } \\
\hline British Technology Center & & & & $\mathbf{x}$ & & & $\mathbf{x}$ & $\mathbf{x}$ \\
\hline \multicolumn{9}{|l|}{ Japan-Oarai Research and Development Center } \\
\hline Plutonium Fuels Research Facility & & & & & & & $\mathbf{x}$ & \\
\hline Alpha Gamma Facility & & & $\mathbf{x}$ & & $\mathbf{x}$ & & $\mathbf{x}$ & \\
\hline Materials Monitoring Facility & & & $\mathbf{x}$ & & $\mathbf{x}$ & & & \\
\hline Sodium Engineering Facility & & & & & & $\mathbf{x}$ & & $\mathbf{x}$ \\
\hline Japan Materials Test Reactor & $\mathbf{x}$ & & & & & & & \\
\hline
\end{tabular}


Table 3-1. (continued).

\begin{tabular}{|c|c|c|c|c|c|c|c|c|c|c|c|}
\hline & \multicolumn{11}{|c|}{ Capabilities } \\
\hline Existing Assets & 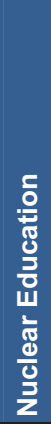 & 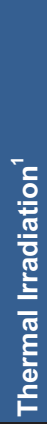 & 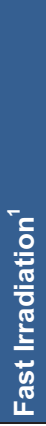 & 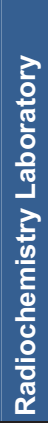 & 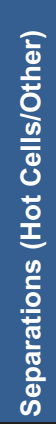 & 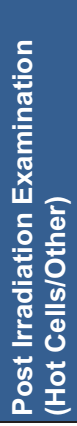 & 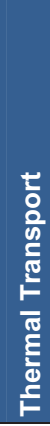 & 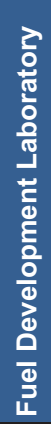 & 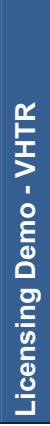 & 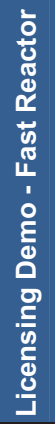 & 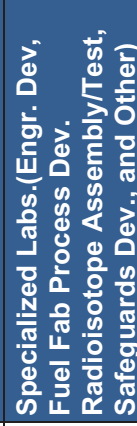 \\
\hline High-Temperature Engineering Test Reactor & & $\mathbf{X}$ & & & & & & & $\mathbf{X}$ & & \\
\hline Fast Reactor Critical Assembly Facility & & $\mathbf{X}$ & & & & & & & & & \\
\hline Hydrogen Production Test Facility & & & & & & & & & & & $\mathbf{X}$ \\
\hline \multicolumn{12}{|l|}{ Japan-Tokai Research and Development Center } \\
\hline Engineering Scale Test and Research Facility & & & & & & & & & & & $\mathbf{X}$ \\
\hline Chemical Processing Facility & & & & $\mathbf{X}$ & $\mathbf{X}$ & & & & & & \\
\hline Recycle Equipment Test Facility & & & & & & & & & & & $\mathbf{X}$ \\
\hline Plutonium Fuels Development Center & & & & & & & & $\mathbf{x}$ & & & \\
\hline Tokai Reprocessing Development Center & & & & $\mathbf{X}$ & $\mathbf{X}$ & & & & & & \\
\hline Nuclear Safety Research Reactor & & $\mathbf{X}$ & $\mathbf{X}$ & & & & & & & & \\
\hline \multicolumn{12}{|l|}{ Japan-Tsuruga } \\
\hline Monju & & & $\mathbf{X}$ & & & & & & & $\mathbf{X}$ & \\
\hline \multicolumn{12}{|l|}{ Russia } \\
\hline BOR-60 & & & $\mathbf{X}$ & & & & & & & & \\
\hline BN600 & & & $\mathbf{x}$ & & & & & & & & \\
\hline BFS-1 and -2 & & $\mathbf{x}$ & & & & & & & & & \\
\hline \multicolumn{12}{|l|}{ Germany } \\
\hline Institute for Transuranium Elements & & & & $\mathbf{x}$ & & & & $\mathbf{X}$ & & & \\
\hline \multicolumn{12}{|l|}{ France-Marcoule } \\
\hline Phenix & & & $\mathbf{x}$ & & & & & & & & \\
\hline Atalante & & & & $\mathbf{x}$ & $\mathbf{X}$ & & & $\mathbf{X}$ & & & \\
\hline \multicolumn{12}{|l|}{ France-Cadarache } \\
\hline Cabri & & $\mathbf{X}$ & & & & & & & & & \\
\hline Phebus & & $\mathbf{X}$ & & & & & & & & & \\
\hline Mascura & & & $\mathbf{X}$ & & & & & & & & \\
\hline Minerve & & $\mathbf{x}$ & $\mathbf{x}$ & & & & & & & & \\
\hline EOLE & & $\mathbf{x}$ & & & & & & & & & \\
\hline AZUR & & $\mathbf{x}$ & & & & & & & & & \\
\hline LEFCA & & & & & & & & $\mathbf{x}$ & & & \\
\hline LECA-STAR & & & & $x$ & & $\mathbf{x}$ & & & & & \\
\hline
\end{tabular}


Table 3-1. (continued).

\begin{tabular}{|c|c|c|c|c|c|c|c|c|c|c|c|}
\hline & \multicolumn{11}{|c|}{ Capabilities } \\
\hline Existing Assets & 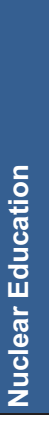 & 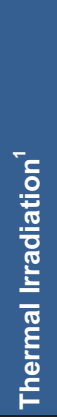 & 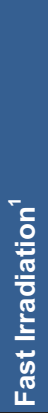 & 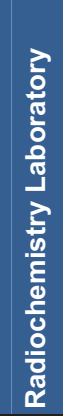 & 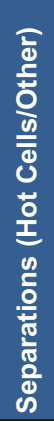 & 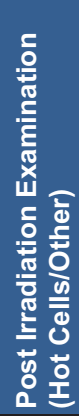 & 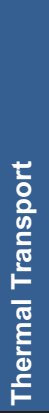 & 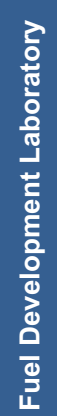 & 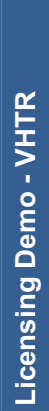 & 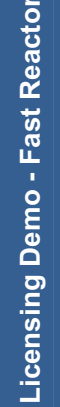 & 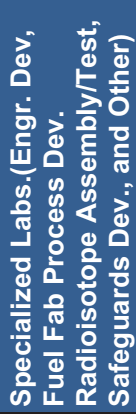 \\
\hline GIAI & & & & & & & $\mathbf{x}$ & & & & \\
\hline \multicolumn{12}{|l|}{ Belgium-Mol } \\
\hline VENUS & & $\mathbf{x}$ & & & & & & & & & \\
\hline \multicolumn{12}{|l|}{ Switzerland_Paul Sherrer Institute } \\
\hline Proteus & & $\mathbf{x}$ & & & & & & & & & \\
\hline Hot Laboratory & & & & & & $\mathbf{x}$ & & & & & \\
\hline
\end{tabular}

1. Includes transient safety testing and physics measurement critical facilities.

2. The Pacific Northwest National Laboratory Physical Sciences Facility is under construction and scheduled for completion in 2011. It will replace the Materials Sciences Laboratory. 3. The partnership includes Boise State University; Idaho State University; University of Idaho; Idaho National Laboratory, and DOE. The Center for Advanced Energy Studies is under construction with an expected completion in the fall of 2008.

\section{Summary of Reviewer Comments}

1. There is no mention of a facility specifically for hydrogen production R\&D.

2. There were numerous comments on additional facilities and modifications to capabilities. In cases where they provided appropriate supplemental information, it is included in Appendix 5.3, Reviewer Supplemental Information and Attachments.

\subsection{Facility Status}

For existing domestic facilities, the status of key parameters is provided via a stoplight color-coded rating system.

\section{Summary of Reviewer Comments}

1. Four additional SRNL facilities and their status were suggested. 


\subsubsection{Facility Assessment Stoplight Color-Coded Rating System}

Tables 3-2 and 3-3 show the decision parameters that were developed for the assessment of facilities.

Table 3-2. Screening and binning rules for assets.

Class 1: Major high-value nuclear facility with attendant support functions. Examples are research, prototype and demonstration nuclear reactors (e.g., Advanced Test Reactor, High Flux Isotope Reactor, and JOYO); large hot-cell facilities (e.g., Hot Fuel Examination Facility) or complex of smaller hot cells (e.g., actinide science and separation laboratories); large multipurpose, multiple capability radiochemistry laboratories; and large glovebox facilities (e.g., TA-55 Plutonium Facility).

Class 2: Nuclear or radiological facility with limited functional capability to support entire mission or major non-radiological facility with nuclear application (e.g., a components test facility); a multipurpose facility with some nuclear application/use (e.g., a high-temperature materials development laboratory).

Class 3: Facilities of a type that are either ubiquitous or would play a modest supporting role in an R\&D program, or which have been removed from consideration by the responsible landlord (e.g., computer clusters, generic non-radiological materials laboratories, and facilities being decommissioned).

Note: Class 1 and Class 2 facilities are included in the evaluation. Class 3 facilities may be listed, but are not included in the evaluation.

Table 3-3. Stoplight rating attributes assessment legend.

Condition - Physical condition, age, and maintenance status of the facility and its supporting infrastructure

Good physical condition with 20 years or more of useful life; capable of performing mission

Capable of performing function with modest investment of about $\$ 25 \mathrm{M}$ or less

Capable of performing most aspects of function after substantial investment of $\$ 25$ to $\$ 250 \mathrm{M}$ over several years

Requires major investment exceeding $\$ 250 \mathrm{M}$

Capability - Capacity, flexibility, location, and accessibility

Proven capability for intended function

Proven capability limited by one or more attributes

Significant limitations for proposed function without major modification

Lacks most needed capabilities for mission

Availability - Projected availability in needed timeframe

Currently available or performing intended function

Has some competing missions but some available capacity; may require operational readiness assessment

Not currently available, fully subscribed by alternate mission; limited lifetime; or requires restart with an operational readiness review

Not available (e.g., currently scheduled for decommissioning and demolition) 
Table 3-3. (continued).

Regulatory Status - Safety basis, environmental impact statement, safety management program, environmental management program, and community support

\begin{tabular}{l}
\hline Fully compliant \\
\hline Can be brought into compliance within 2 years with an investment of $\$ 5 \mathrm{M}$ or less \\
\hline $\begin{array}{l}\text { Significant compliance issues that requires more than } 2 \text { years and sustained investment of several } \\
\text { million dollars per year }\end{array}$ \\
Serious safety and environmental liability
\end{tabular}

Safeguards and Security - DOE security requirements for type of facility and materials handled: PIDAS, guard force, nuclear materials management system, and cyber security (considers safeguards and security requirements as of April 2008)

Compliant with current safeguards and security requirements and has implementation plan for emerging requirements

Compliant with current requirements; significant effort to meet emerging design basis threat

Unable to meet security requirements for mission without substantial capital and annual investment

Unable to meet security requirements because of unfixable conditions such as proximity to public areas

Human Capital - Requisite skills including R\&D, operations, maintenance, and support personnel onsite or readily available

Fully staffed with no projected cuts in critical skills

All required skills available, but augmentation needed to perform mission and staffing plan needed to deal with critical retirement issues

Some but not all critical skills available for mission

Requires essentially completely new workforce

At this stage, it is crucial to understand that the color-coded ratings are only an indicator of how much effort will be required to modify a facility to perform an identified R\&D function. Some facilities with "favorable" colors (green and yellow) may be adequate for only a limited scope of program support. Large facilities will have more challenging requirements, and therefore, more gaps indicated by orange or red, but may still be cost-effective options relative to other alternatives or new infrastructure. Even "green" facilities require sustained investment for maintenance and upgrading.

\subsubsection{Light Water Reactors}

It should be noted that facility attributes indicated in a red or orange color in the stoplight color-coded rating system will require a substantial or major investment to restore them to a fully functional state; however, those investments may still be cost-effective options. Table 3-4 provides a summary reminder of the rating system's assessment designators and Table 3-5 shows the facility assessments for light water reactor R\&D support. 
NOTE: Stoplight assessments in the following tables are preliminary and have not been validated. Parameters are assessed for an expected 20 years of future operation.

Table 3-4. Summary example of the facility stoplight color-coded rating system.

\begin{tabular}{|l|l|}
\hline Green & Mission ready \\
\hline Yellow & Ready within 2 years or less with modest investment \\
\hline Orange & Needs modification or upgrades that require significant investment \\
\hline Red & Usable only after major capital investment over an extended number of years \\
\hline
\end{tabular}

Table 3-5. Facilities assessed for light water reactor research and development support.

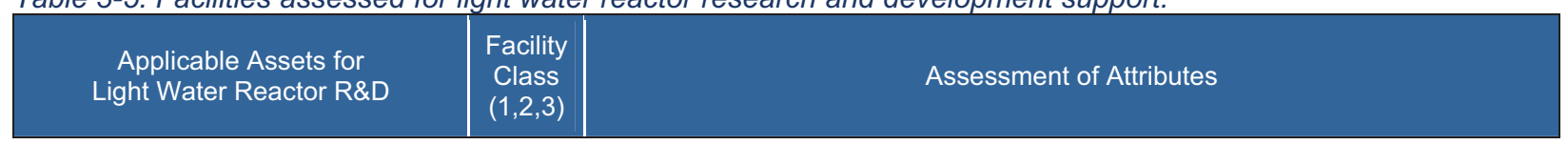

\section{Irradiation Facilities}

\begin{tabular}{|l|c|l|l|l|l|l|l|}
\hline $\begin{array}{l}\text { Advanced Test Reactor, Idaho } \\
\text { National Laboratory }\end{array}$ & $\mathbf{1}$ & Condition & Capability & Availability & $\begin{array}{l}\text { Regulatory } \\
\text { Status }\end{array}$ & $\begin{array}{l}\text { Safeguards } \\
\text { and Security }\end{array}$ & $\begin{array}{l}\text { Human } \\
\text { Capital }\end{array}$ \\
\hline $\begin{array}{l}\text { High Flux Isotope Reactor, Oak } \\
\text { Ridge National Laboratory }\end{array}$ & $\mathbf{1}$ & Condition & Capability & Availability & $\begin{array}{l}\text { Regulatory } \\
\text { Status }\end{array}$ & $\begin{array}{l}\text { Safeguards } \\
\text { and Security }\end{array}$ & $\begin{array}{l}\text { Human } \\
\text { Capital }\end{array}$ \\
\hline $\begin{array}{l}\text { Transient Reactor Test Facility, } \\
\text { Idaho National Laboratory }\end{array}$ & 1 & Condition & Capability & Availability & $\begin{array}{l}\text { Regulatory } \\
\text { Status }\end{array}$ & $\begin{array}{l}\text { Safeguards } \\
\text { and Security }\end{array}$ & $\begin{array}{l}\text { Human } \\
\text { Capital }\end{array}$ \\
\hline
\end{tabular}

\section{Hot Cells}

\begin{tabular}{|c|c|c|c|c|c|c|c|}
\hline $\begin{array}{l}\text { Hot Fuel Examination Facility, Idaho } \\
\text { National Laboratory }\end{array}$ & 1 & Condition & Capability & Availability & $\begin{array}{l}\text { Regulatory } \\
\text { Status }\end{array}$ & $\begin{array}{l}\text { Safeguards } \\
\text { and Security }\end{array}$ & $\begin{array}{l}\text { Human } \\
\text { Capital }\end{array}$ \\
\hline $\begin{array}{l}\text { Irradiated Fuels Exam Laboratory, } \\
\text { Oak Ridge National Laboratory }\end{array}$ & 1 & Condition & Capability & Availability & $\begin{array}{l}\text { Regulatory } \\
\text { Status }\end{array}$ & $\begin{array}{l}\text { Safeguards } \\
\text { and Security }\end{array}$ & $\begin{array}{l}\text { Human } \\
\text { Capital }\end{array}$ \\
\hline $\begin{array}{l}\text { Irradiated Materials Examination and } \\
\text { Testing Facility, Oak Ridge National } \\
\text { Laboratory }\end{array}$ & 2 & Condition & Capability & Availability & $\begin{array}{l}\text { Regulatory } \\
\text { Status }\end{array}$ & $\begin{array}{l}\text { Safeguards } \\
\text { and Security }\end{array}$ & $\begin{array}{l}\text { Human } \\
\text { Capital }\end{array}$ \\
\hline
\end{tabular}

\section{Radiochemistry Laboratories}

\begin{tabular}{|c|c|c|c|c|c|c|c|}
\hline $\begin{array}{l}\text { Materials and Fuels Complex } \\
\text { Analytical Laboratory, Idaho National } \\
\text { Laboratory }\end{array}$ & 1 & Condition & Capability & Availability & $\begin{array}{l}\text { Regulatory } \\
\text { Status }\end{array}$ & $\begin{array}{l}\text { Safeguards } \\
\text { and Security }\end{array}$ & $\begin{array}{l}\text { Human } \\
\text { Capital }\end{array}$ \\
\hline
\end{tabular}

\section{Summary of Reviewer Comments}

1. Facilities suggested for addition included: ORNL RAG laboratories, LAMDA; SNL ACRR, SPRF and Gamma Irradiation Facility

2. There were numerous suggestions for changing the color ratings of specific facilities. 


\subsubsection{Irradiated Fuel Separations}

It should be noted that facility attributes indicated in a red or orange color in the stoplight color-coded rating system will require a substantial or major investment to restore them to a fully functional state; however, those investments may still be cost-effective options. Table 3-6 provides a summary reminder of the rating system's assessment designators and Table 3-7 shows the facility assessments for irradiated fuel separations R\&D support.

NOTE: Stoplight assessments in the following tables are preliminary and have not been validated. Parameters are assessed for an expected 20 years of future operation.

Table 3-6. Summary example of the facility stoplight color-coded rating system.

\begin{tabular}{|l|l|}
\hline Green & Mission ready \\
\hline Yellow & Ready within 2 years or less with modest investment \\
\hline Orange & Needs modification or upgrades that require significant investment \\
\hline Red & Usable only after major capital investment over an extended number of years \\
\hline
\end{tabular}

Table 3-7. Facilities assessed for irradiated fuel separations research and development support.

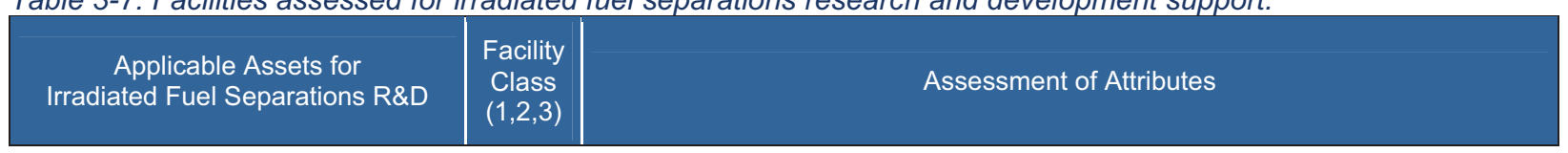

\section{Hot Cells}

\begin{tabular}{|c|c|c|c|c|c|c|c|}
\hline $\begin{array}{l}\text { Fluorinel Fuel Dissolution Facility, } \\
\text { CPP-666, Idaho National Laboratory }\end{array}$ & 1 & Condition & Capability & Availability & $\begin{array}{l}\text { Regulatory } \\
\text { Status }\end{array}$ & $\begin{array}{l}\text { Safeguards } \\
\text { and Security }\end{array}$ & $\begin{array}{l}\text { Human } \\
\text { Capital }\end{array}$ \\
\hline $\begin{array}{l}\text { Fuel Conditioning Facility, Idaho } \\
\text { National Laboratory }\end{array}$ & 1 & Condition & Capability & Availability & $\begin{array}{l}\text { Regulatory } \\
\text { Status }\end{array}$ & $\begin{array}{l}\text { Safeguards } \\
\text { and Security }\end{array}$ & $\begin{array}{l}\text { Human } \\
\text { Capital }\end{array}$ \\
\hline $\begin{array}{l}\text { Fuel Processing Facility, CPP-691, } \\
\text { Idaho National Laboratory }\end{array}$ & 1 & Condition & Capability & Availability & $\begin{array}{l}\text { Regulatory } \\
\text { Status }\end{array}$ & $\begin{array}{l}\text { Safeguards } \\
\text { and Security }\end{array}$ & $\begin{array}{l}\text { Human } \\
\text { Capital }\end{array}$ \\
\hline $\begin{array}{l}\text { Fuels and Materials Examination } \\
\text { Facility, Pacific Northwest National } \\
\text { Laboratory }\end{array}$ & 1 & Condition & Capability & Availability & $\begin{array}{l}\text { Regulatory } \\
\text { Status }\end{array}$ & $\begin{array}{l}\text { Safeguards } \\
\text { and Security }\end{array}$ & $\begin{array}{l}\text { Human } \\
\text { Capital }\end{array}$ \\
\hline $\begin{array}{l}\text { H-Canyon, Savannah River National } \\
\text { Laboratory }\end{array}$ & 1 & Condition & Capability & Availability & $\begin{array}{l}\text { Regulatory } \\
\text { Status }\end{array}$ & $\begin{array}{l}\text { Safeguards } \\
\text { and Security }\end{array}$ & $\begin{array}{l}\text { Human } \\
\text { Capital }\end{array}$ \\
\hline $\begin{array}{l}\text { Hot Fuel Examination Facility, Idaho } \\
\text { National Laboratory }\end{array}$ & 1 & Condition & Capability & Availability & $\begin{array}{l}\text { Regulatory } \\
\text { Status }\end{array}$ & $\begin{array}{l}\text { Safeguards } \\
\text { and Security }\end{array}$ & $\begin{array}{l}\text { Human } \\
\text { Capital }\end{array}$ \\
\hline $\begin{array}{l}\text { Irradiated Fuels Examination } \\
\text { Laboratory, Oak Ridge National } \\
\text { Laboratory }\end{array}$ & 2 & Condition & Capability & Availability & $\begin{array}{l}\text { Regulatory } \\
\text { Status }\end{array}$ & $\begin{array}{l}\text { Safeguards } \\
\text { and Security }\end{array}$ & $\begin{array}{l}\text { Human } \\
\text { Capital }\end{array}$ \\
\hline $\begin{array}{l}\text { Radiochemical Engineering } \\
\text { Development Center (REDC-7920), } \\
\text { Oak Ridge National Laboratory }\end{array}$ & 1 & Condition & Capability & Availability & $\begin{array}{l}\text { Regulatory } \\
\text { Status }\end{array}$ & $\begin{array}{l}\text { Safeguards } \\
\text { and Security }\end{array}$ & $\begin{array}{l}\text { Human } \\
\text { Capital }\end{array}$ \\
\hline $\begin{array}{l}\text { Radiochemical Engineering } \\
\text { Development Center (REDC-7930), } \\
\text { Oak Ridge National Laboratory }\end{array}$ & 1 & Condition & Capability & Availability & $\begin{array}{l}\text { Regulatory } \\
\text { Status }\end{array}$ & $\begin{array}{l}\text { Safeguards } \\
\text { and Security }\end{array}$ & $\begin{array}{l}\text { Human } \\
\text { Capital }\end{array}$ \\
\hline $\begin{array}{l}\text { Radiochemical Processing } \\
\text { Laboratory, Pacific Northwest } \\
\text { National Laboratory }\end{array}$ & 2 & Condition & Capability & Availability & $\begin{array}{l}\text { Regulatory } \\
\text { Status }\end{array}$ & $\begin{array}{l}\text { Safeguards } \\
\text { and Security }\end{array}$ & $\begin{array}{l}\text { Human } \\
\text { Capital }\end{array}$ \\
\hline
\end{tabular}


Table 3-7. (continued).

\begin{tabular}{|l|c|c|l|l|l|l|l|}
\hline $\begin{array}{c}\text { Applicable Assets for } \\
\text { Irradiated Fuel Separations R\&D }\end{array}$ & $\begin{array}{c}\text { Facility } \\
\text { Class } \\
(1,2,3)\end{array}$ & \multicolumn{5}{|c|}{ Assessment of Attributes } \\
\hline $\begin{array}{l}\text { Shielded Cell Facility, Savannah } \\
\text { River National Laboratory }\end{array}$ & $\mathbf{2}$ & Condition & Capability & Availability & $\begin{array}{l}\text { Regulatory } \\
\text { Status }\end{array}$ & $\begin{array}{l}\text { Safeguards } \\
\text { and Security }\end{array}$ & $\begin{array}{l}\text { Human } \\
\text { Capital }\end{array}$ \\
\hline
\end{tabular}

\section{Radiochemistry Laboratories}

\begin{tabular}{|l|c|l|l|l|l|l|l|}
\hline $\begin{array}{l}\text { Materials and Fuels Complex } \\
\text { Analytical Laboratory, Idaho National } \\
\text { Laboratory }\end{array}$ & $\mathbf{1}$ & Condition & Capability & Availability & $\begin{array}{l}\text { Regulatory } \\
\text { Status }\end{array}$ & $\begin{array}{l}\text { Safeguards } \\
\text { and Security }\end{array}$ & $\begin{array}{l}\text { Human } \\
\text { Capital }\end{array}$ \\
\hline $\begin{array}{l}\text { Remote Analytical Laboratory, } \\
\text { CPP-684, Idaho National Laboratory }\end{array}$ & $\mathbf{2}$ & Condition & Capability & Availability & $\begin{array}{l}\text { Regulatory } \\
\text { Status }\end{array}$ & $\begin{array}{l}\text { Safeguards } \\
\text { and Security }\end{array}$ & $\begin{array}{l}\text { Human } \\
\text { Capital }\end{array}$ \\
\hline
\end{tabular}

\section{Specialized Laboratory Facilities}

\begin{tabular}{|l|c|l|l|l|l|l|l|}
\hline $\begin{array}{l}\text { Fluorinel Fuel Storage Facility, } \\
\text { CPP-666, Idaho National Laboratory }\end{array}$ & $\mathbf{2}$ & Condition & Capability & Availability & $\begin{array}{l}\text { Regulatory } \\
\text { Status }\end{array}$ & $\begin{array}{l}\text { Safeguards } \\
\text { and Security }\end{array}$ & $\begin{array}{l}\text { Human } \\
\text { Capital }\end{array}$ \\
\hline $\begin{array}{l}\text { New Waste Calcine Facility, } \\
\text { CPP-659, Idaho National Laboratory }\end{array}$ & $\mathbf{2}$ & Condition & Capability & Availability & $\begin{array}{l}\text { Regulatory } \\
\text { Status }\end{array}$ & $\begin{array}{l}\text { Safeguards } \\
\text { and Security }\end{array}$ & $\begin{array}{l}\text { Human } \\
\text { Capital }\end{array}$ \\
\hline $\begin{array}{l}\text { Receiving Basin for Offsite Fuel, } \\
\text { Savannah River National Laboratory }\end{array}$ & $\mathbf{2}$ & Condition & Capability & Availability & $\begin{array}{l}\text { Regulatory } \\
\text { Status }\end{array}$ & $\begin{array}{l}\text { Safeguards } \\
\text { and Security }\end{array}$ & $\begin{array}{l}\text { Human } \\
\text { Capital }\end{array}$ \\
\hline $\begin{array}{l}\text { Unirradiated Fuel Storage Facility, } \\
\text { CPP-651, Idaho National Laboratory }\end{array}$ & $\mathbf{2}$ & Condition & Capability & Availability & $\begin{array}{l}\text { Regulatory } \\
\text { Status }\end{array}$ & $\begin{array}{l}\text { Safeguards } \\
\text { and Security }\end{array}$ & $\begin{array}{l}\text { Human } \\
\text { Capital }\end{array}$ \\
\hline
\end{tabular}

3.2.3.1 Other Considered Facilities. Other facilities identified as capable of playing a limited support role (i.e., Class 3 facilities) for irradiated fuel separations R\&D include the following:

- $\quad$ TA-48 Radiochemistry Laboratory, Los Alamos National Laboratory

- TA-55, PF-4 Los Alamos National Laboratory

- $\quad 205$ Chemical Engineering Building, Argonne National Laboratory

- $\quad$ Engineering Development Laboratory, Idaho National Laboratory

- $\quad$ Engineering Development Laboratory, Savannah River National Laboratory

- J, G, K, and M Wing Facilities, Argonne National Laboratory.

\section{Summary of Reviewer Comments}

1. Add the ORNL RAG laboratories.

2. There were numerous suggestions for modifications to color ratings of specific facilities. 


\subsubsection{Advanced Fuel Development}

It should be noted that facility attributes indicated in a red or orange color in the stoplight color-coded rating system will require a substantial or major investment to restore them to a fully functional state; however, those investments may still be cost-effective options. Table 3-8 provides a summary reminder of the rating system's assessment designators and Table 3-9 shows the facility assessments for advanced fuel development R\&D support.

NOTE: Stoplight assessments in the following tables are preliminary and have not been validated. Parameters are assessed for an expected 20 years of future operation.

Table 3-8. Summary example of the facility stoplight color-coded rating system.

\begin{tabular}{|l|l|}
\hline Green & Mission ready \\
\hline Yellow & Ready within 2 years or less with modest investment \\
\hline Orange & Needs modification or upgrades that require significant investment \\
\hline Red & Usable only after major capital investment over an extended number of years \\
\hline
\end{tabular}

Table 3-9. Facilities assessed for advanced fuel development research and development support.

\begin{tabular}{|c|l|l|}
\hline Applicable Assets for & $\begin{array}{l}\text { Facility } \\
\text { Class } \\
(1,2,3)\end{array}$ & Assessment of Attributes \\
Advanced Fuel Development R\&D & \\
\hline
\end{tabular}

\section{Irradiation Facilities}

\begin{tabular}{|l|c|l|l|l|l|l|l|}
\hline $\begin{array}{l}\text { Advanced Test Reactor, Idaho } \\
\text { National Laboratory }\end{array}$ & $\mathbf{1}$ & Condition & Capability & Availability & $\begin{array}{l}\text { Regulatory } \\
\text { Status }\end{array}$ & $\begin{array}{l}\text { Safeguards } \\
\text { and Security }\end{array}$ & $\begin{array}{l}\text { Human } \\
\text { Capital }\end{array}$ \\
\hline $\begin{array}{l}\text { Annular Core Research Reactor, } \\
\text { Sandia National Laboratory }\end{array}$ & $\mathbf{1}$ & Condition & Capability & Availability & $\begin{array}{l}\text { Regulatory } \\
\text { Status }\end{array}$ & $\begin{array}{l}\text { Safeguards } \\
\text { and Security }\end{array}$ & $\begin{array}{l}\text { Human } \\
\text { Capital }\end{array}$ \\
\hline $\begin{array}{l}\text { Fast Flux Test Facility, Richland, } \\
\text { Washington }\end{array}$ & $\mathbf{1}$ & Condition & Capability & Availability & $\begin{array}{l}\text { Regulatory } \\
\text { Status }\end{array}$ & $\begin{array}{l}\text { Safeguards } \\
\text { and Security }\end{array}$ & $\begin{array}{l}\text { Human } \\
\text { Capital }\end{array}$ \\
\hline $\begin{array}{l}\text { High Flux Isotope Reactor, Oak } \\
\text { Ridge National Laboratory }\end{array}$ & $\mathbf{1}$ & Condition & Capability & Availability & $\begin{array}{l}\text { Regulatory } \\
\text { Status }\end{array}$ & $\begin{array}{l}\text { Safeguards } \\
\text { and Security }\end{array}$ & $\begin{array}{l}\text { Human } \\
\text { Capital }\end{array}$ \\
\hline $\begin{array}{l}\text { Transient Reactor Test Facility, } \\
\text { Idaho National Laboratory }\end{array}$ & $\mathbf{1}$ & Condition & Capability & Availability & $\begin{array}{l}\text { Regulatory } \\
\text { Status }\end{array}$ & $\begin{array}{l}\text { Safeguards } \\
\text { and Security }\end{array}$ & $\begin{array}{l}\text { Human } \\
\text { Capital }\end{array}$ \\
\hline
\end{tabular}


Table 3-9. (continued).

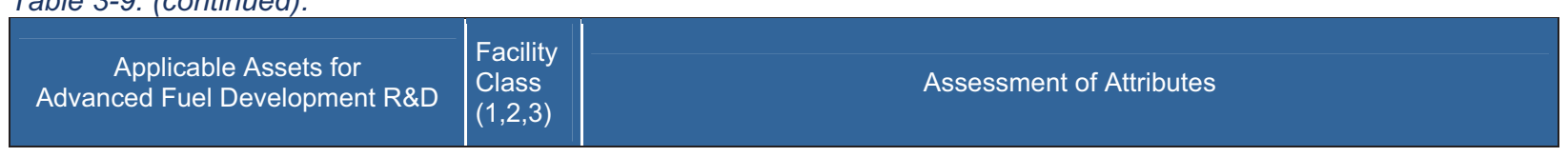

Hot Cells

\begin{tabular}{|c|c|c|c|c|c|c|c|}
\hline $\begin{array}{l}\text { Chemistry and Metallurgical } \\
\text { Research Facility Wing 9, Los } \\
\text { Alamos National Laboratory }\end{array}$ & 1 & Condition & Capability & Availability & $\begin{array}{l}\text { Regulatory } \\
\text { Status }\end{array}$ & $\begin{array}{l}\text { Safeguards } \\
\text { and Security }\end{array}$ & $\begin{array}{l}\text { Human } \\
\text { Capital }\end{array}$ \\
\hline $\begin{array}{l}\text { Fuel Conditioning Facility, Idaho } \\
\text { National Laboratory }\end{array}$ & 1 & Condition & Capability & Availability & $\begin{array}{l}\text { Regulatory } \\
\text { Status }\end{array}$ & $\begin{array}{l}\text { Safeguards } \\
\text { and Security }\end{array}$ & $\begin{array}{l}\text { Human } \\
\text { Capital }\end{array}$ \\
\hline $\begin{array}{l}\text { Fuels and Materials Examination } \\
\text { Facility, Pacific Northwest National } \\
\text { Laboratory }\end{array}$ & 1 & Condition & Capability & Availability & $\begin{array}{l}\text { Regulatory } \\
\text { Status }\end{array}$ & $\begin{array}{l}\text { Safeguards } \\
\text { and Security }\end{array}$ & $\begin{array}{l}\text { Human } \\
\text { Capital }\end{array}$ \\
\hline $\begin{array}{l}\text { H-Canyon, Savannah River National } \\
\text { Laboratory }\end{array}$ & 2 & Condition & Capability & Availability & $\begin{array}{l}\text { Regulatory } \\
\text { Status }\end{array}$ & $\begin{array}{l}\text { Safeguards } \\
\text { and Security }\end{array}$ & $\begin{array}{l}\text { Human } \\
\text { Capital }\end{array}$ \\
\hline $\begin{array}{l}\text { Hot Fuel Examination Facility, Idaho } \\
\text { National Laboratory }\end{array}$ & 1 & Condition & Capability & Availability & $\begin{array}{l}\text { Regulatory } \\
\text { Status }\end{array}$ & $\begin{array}{l}\text { Safeguards } \\
\text { and Security }\end{array}$ & $\begin{array}{l}\text { Human } \\
\text { Capital }\end{array}$ \\
\hline $\begin{array}{l}\text { Irradiated Fuels Examination } \\
\text { Laboratory, Oak Ridge National } \\
\text { Laboratory }\end{array}$ & 1 & Condition & Capability & Availability & $\begin{array}{l}\text { Regulatory } \\
\text { Status }\end{array}$ & $\begin{array}{l}\text { Safeguards } \\
\text { and Security }\end{array}$ & $\begin{array}{l}\text { Human } \\
\text { Capital }\end{array}$ \\
\hline $\begin{array}{l}\text { Radiochemical Engineering } \\
\text { Development Center (REDC-7920), } \\
\text { Oak Ridge National Laboratory }\end{array}$ & 1 & Condition & Capability & Availability & $\begin{array}{l}\text { Regulatory } \\
\text { Status }\end{array}$ & $\begin{array}{l}\text { Safeguards } \\
\text { and Security }\end{array}$ & $\begin{array}{l}\text { Human } \\
\text { Capital }\end{array}$ \\
\hline $\begin{array}{l}\text { Radiochemical Engineering } \\
\text { Development Center (REDC-7930), } \\
\text { Oak Ridge National Laboratory }\end{array}$ & 1 & Condition & Capability & Availability & $\begin{array}{l}\text { Regulatory } \\
\text { Status }\end{array}$ & $\begin{array}{l}\text { Safeguards } \\
\text { and Security }\end{array}$ & $\begin{array}{l}\text { Human } \\
\text { Capital }\end{array}$ \\
\hline $\begin{array}{l}\text { Radiochemical Processing } \\
\text { Laboratory, Pacific Northwest } \\
\text { National Laboratory }\end{array}$ & 2 & Condition & Capability & Availability & $\begin{array}{l}\text { Regulatory } \\
\text { Status }\end{array}$ & $\begin{array}{l}\text { Safeguards } \\
\text { and Security }\end{array}$ & $\begin{array}{l}\text { Human } \\
\text { Capital }\end{array}$ \\
\hline $\begin{array}{l}\text { Shielded Cell Facility, Savannah } \\
\text { River National Laboratory }\end{array}$ & 2 & Condition & Capability & Availability & $\begin{array}{l}\text { Regulatory } \\
\text { Status }\end{array}$ & $\begin{array}{l}\text { Safeguards } \\
\text { and Security }\end{array}$ & $\begin{array}{l}\text { Human } \\
\text { Capital }\end{array}$ \\
\hline
\end{tabular}

\section{Radiochemistry Laboratories}

\begin{tabular}{|c|c|c|c|c|c|c|c|}
\hline $\begin{array}{l}\text { Chemistry and Metallurgical } \\
\text { Research Wings } 5 \text { and } 7 \text { Analytical } \\
\text { Chemistry Laboratory, Los Alamos } \\
\text { National Laboratory }\end{array}$ & 2 & Condition & Capability & Availability & $\begin{array}{l}\text { Regulatory } \\
\text { Status }\end{array}$ & $\begin{array}{l}\text { Safeguards } \\
\text { and Security }\end{array}$ & $\begin{array}{l}\text { Human } \\
\text { Capital }\end{array}$ \\
\hline $\begin{array}{l}\text { Materials and Fuels Complex } \\
\text { Analytical Laboratory, Idaho National } \\
\text { Laboratory }\end{array}$ & 1 & Condition & Capability & Availability & $\begin{array}{l}\text { Regulatory } \\
\text { Status }\end{array}$ & $\begin{array}{l}\text { Safeguards } \\
\text { and Security }\end{array}$ & $\begin{array}{l}\text { Human } \\
\text { Capital }\end{array}$ \\
\hline $\begin{array}{l}\text { Remote Analytical Laboratory, } \\
\text { CPP-684, Idaho National Laboratory }\end{array}$ & 2 & Condition & Capability & Availability & $\begin{array}{l}\text { Regulatory } \\
\text { Status }\end{array}$ & $\begin{array}{l}\text { Safeguards } \\
\text { and Security }\end{array}$ & $\begin{array}{l}\text { Human } \\
\text { Capital }\end{array}$ \\
\hline
\end{tabular}

\section{Fuel Development Facilities}

\begin{tabular}{|l|c|l|l|l|l|l|l|}
\hline $\begin{array}{l}\text { Fuel Manufacturing Facility, Idaho } \\
\text { National Laboratory }\end{array}$ & $\mathbf{1}$ & Condition & Capability & Availability & $\begin{array}{l}\text { Regulatory } \\
\text { Status }\end{array}$ & $\begin{array}{l}\text { Safeguards } \\
\text { and Security }\end{array}$ & $\begin{array}{l}\text { Human } \\
\text { Capital }\end{array}$ \\
\hline $\begin{array}{l}\text { TA-55, PF-4 Los Alamos National } \\
\text { Laboratory }\end{array}$ & $\mathbf{1}$ & Condition & Capability & Availability & $\begin{array}{l}\text { Regulatory } \\
\text { Status }\end{array}$ & $\begin{array}{l}\text { Safeguards } \\
\text { and Security }\end{array}$ & $\begin{array}{l}\text { Human } \\
\text { Capital }\end{array}$ \\
\hline
\end{tabular}


Table 3-9. (continued).

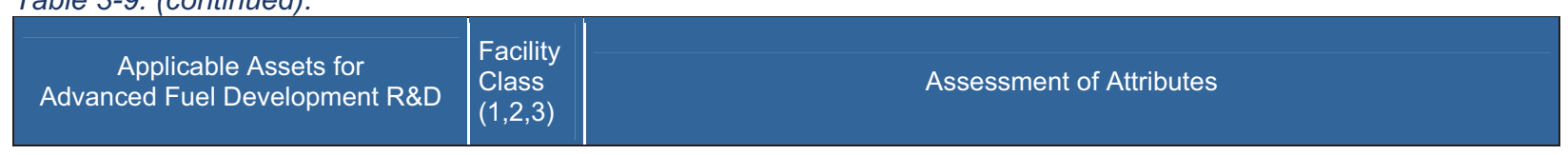

\section{Specialized Laboratory Facilities}

\begin{tabular}{|l|c|l|l|l|l|l|l|}
\hline $\begin{array}{l}\text { Electron Microscopy Laboratory, } \\
\text { Idaho National Laboratory }\end{array}$ & $\mathbf{2}$ & Condition & Capability & Availability & $\begin{array}{l}\text { Regulatory } \\
\text { Status }\end{array}$ & $\begin{array}{l}\text { Safeguards } \\
\text { and Security }\end{array}$ & $\begin{array}{l}\text { Human } \\
\text { Capital }\end{array}$ \\
\hline $\begin{array}{l}\text { Fuel Assembly and Storage Building, } \\
\text { Idaho National Laboratory }\end{array}$ & $\mathbf{2}$ & Condition & Capability & Availability & $\begin{array}{l}\text { Regulatory } \\
\text { Status }\end{array}$ & $\begin{array}{l}\text { Safeguards } \\
\text { and Security }\end{array}$ & $\begin{array}{l}\text { Human } \\
\text { Capital }\end{array}$ \\
\hline $\begin{array}{l}\text { Unirradiated Fuel Storage Facility, } \\
\text { CPP-651, Idaho National Laboratory }\end{array}$ & $\mathbf{2}$ & Condition & Capability & Availability & $\begin{array}{l}\text { Regulatory } \\
\text { Status }\end{array}$ & $\begin{array}{l}\text { Safeguards } \\
\text { and Security }\end{array}$ & $\begin{array}{l}\text { Human } \\
\text { Capital }\end{array}$ \\
\hline
\end{tabular}

3.2.4.1 Proposed Facilities. Materials Test Station, TA-53, (LANCE), Los Alamos National Laboratory.

3.2.4.2 Other Considered Facilities. Other facilities identified as capable of playing a limited support role (i.e., Class 3 facilities) for advanced fuel development R\&D include the following:

- $\quad$ TA-48 Radiochemistry Laboratory, Los Alamos National Laboratory

- $\quad 205$ Chemical Engineering Building, Argonne National Laboratory

- $\quad$ Engineering Development Laboratory, Idaho National Laboratory

- $\quad$ Engineering Development Laboratory, Savannah River National Laboratory

- $\quad \mathrm{J}, \mathrm{G}, \mathrm{K}$, and M Wing Facilities, Argonne National Laboratory.

\section{Summary of Reviewer Comments}

1. Add ORNL RAG and TRISO laboratories

2. There were numerous suggestions for modifications to color ratings of specific facilities.

\subsubsection{High-Temperature Reactor}

It should be noted that facility attributes indicated in a red or orange color in the stoplight color-coded rating system will require a substantial or major investment to restore them to a fully functional state; however, those investments may still be cost-effective options. Table 3-10 provides a summary reminder of the rating system's assessment designators and Table 3-11 shows the facility assessments for high-temperature reactor R\&D support.

NOTE: Stoplight assessments in the following tables are preliminary and have not been validated. Parameters are assessed for an expected 20 years of future operation. 
Table 3-10. Summary example of the facility stoplight color-coded rating system.

\begin{tabular}{|l|l|}
\hline Green & Mission ready \\
\hline Yellow & Ready within 2 years or less with modest investment \\
\hline Orange & Needs modification or upgrades that require significant investment \\
\hline Red & Usable only after major capital investment over an extended number of years \\
\hline
\end{tabular}

Table 3-11. Facilities assessed for high-temperature reactor research and development support.

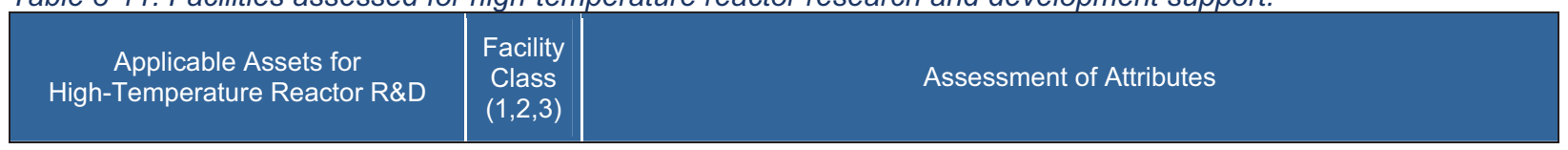

\section{Irradiation Facilities}

\begin{tabular}{|l|c|l|l|l|l|l|l|}
\hline $\begin{array}{l}\text { Advanced Test Reactor, Idaho } \\
\text { National Laboratory }\end{array}$ & $\mathbf{1}$ & Condition & Capability & Availability & $\begin{array}{l}\text { Regulatory } \\
\text { Status }\end{array}$ & $\begin{array}{l}\text { Safeguards } \\
\text { and Security }\end{array}$ & $\begin{array}{l}\text { Human } \\
\text { Capital }\end{array}$ \\
\hline $\begin{array}{l}\text { High Flux Isotope Reactor, Oak } \\
\text { Ridge National Laboratory }\end{array}$ & $\mathbf{1}$ & Condition & Capability & Availability & $\begin{array}{l}\text { Regulatory } \\
\text { Status }\end{array}$ & $\begin{array}{l}\text { Safeguards } \\
\text { and Security }\end{array}$ & $\begin{array}{l}\text { Human } \\
\text { Capital }\end{array}$ \\
\hline $\begin{array}{l}\text { Neutron Radiography Reactor } \\
\text { Facility (Hot Fuel Examination } \\
\text { Facility), Idaho National Laboratory }\end{array}$ & $\mathbf{1}$ & Condition & Capability & Availability & $\begin{array}{l}\text { Regulatory } \\
\text { Status }\end{array}$ & $\begin{array}{l}\text { Safeguards } \\
\text { and Security }\end{array}$ & $\begin{array}{l}\text { Human } \\
\text { Capital }\end{array}$ \\
\hline
\end{tabular}

\section{Hot Cells}

\begin{tabular}{|l|c|l|l|l|l|l|l|}
\hline $\begin{array}{l}\text { Hot Fuel Examination Facility, Idaho } \\
\text { National Laboratory }\end{array}$ & $\mathbf{1}$ & Condition & Capability & Availability & $\begin{array}{l}\text { Regulatory } \\
\text { Status }\end{array}$ & $\begin{array}{l}\text { Safeguards } \\
\text { and Security }\end{array}$ & $\begin{array}{l}\text { Human } \\
\text { Capital }\end{array}$ \\
\hline $\begin{array}{l}\text { Irradiated Fuels Examination } \\
\text { Laboratory, Oak Ridge National } \\
\text { Laboratory }\end{array}$ & $\mathbf{1}$ & Condition & Capability & Availability & $\begin{array}{l}\text { Regulatory } \\
\text { Status }\end{array}$ & $\begin{array}{l}\text { Safeguards } \\
\text { and Security }\end{array}$ & $\begin{array}{l}\text { Human } \\
\text { Capital }\end{array}$ \\
\hline
\end{tabular}

\section{Radiochemistry Laboratories}

\begin{tabular}{|c|c|c|c|c|c|c|c|}
\hline $\begin{array}{l}\text { Radiochemical Analysis } \\
\text { Laboratories, Idaho National } \\
\text { Laboratory }\end{array}$ & 1 & Condition & Capability & Availability & $\begin{array}{l}\text { Regulatory } \\
\text { Status }\end{array}$ & $\begin{array}{l}\text { Safeguards } \\
\text { and Security }\end{array}$ & $\begin{array}{l}\text { Human } \\
\text { Capital }\end{array}$ \\
\hline $\begin{array}{l}\text { Radiochemical Analysis } \\
\text { Laboratories, Oak Ridge National } \\
\text { Laboratory }\end{array}$ & 1 & Condition & Capability & Availability & $\begin{array}{l}\text { Regulatory } \\
\text { Status }\end{array}$ & $\begin{array}{l}\text { Safeguards } \\
\text { and Security }\end{array}$ & $\begin{array}{l}\text { Human } \\
\text { Capital }\end{array}$ \\
\hline $\begin{array}{l}\text { High-Level Chemical Development } \\
\text { Laboratory (Building 4507), Oak } \\
\text { Ridge National Laboratory }\end{array}$ & 2 & Condition & Capability & Availability & $\begin{array}{l}\text { Regulatory } \\
\text { Status }\end{array}$ & $\begin{array}{l}\text { Safeguards } \\
\text { and Security }\end{array}$ & $\begin{array}{l}\text { Human } \\
\text { Capital }\end{array}$ \\
\hline
\end{tabular}

\section{Fuel Development Facilities}

\begin{tabular}{|c|c|c|c|c|c|c|c|}
\hline $\begin{array}{l}\text { Babcock \& Wilcox (B\&W) Fuel } \\
\text { Fabrication Facility, Commercial }\end{array}$ & 1 & Condition & Capability & Availability & $\begin{array}{l}\text { Regulatory } \\
\text { Status }\end{array}$ & $\begin{array}{l}\text { Safeguards } \\
\text { and Security }\end{array}$ & $\begin{array}{l}\text { Human } \\
\text { Capital }\end{array}$ \\
\hline $\begin{array}{l}\text { Sol Gel Laboratory Facility (Building } \\
\text { 4501), Oak Ridge National } \\
\text { Laboratory }\end{array}$ & 2 & Condition & Capability & Availability & $\begin{array}{l}\text { Regulatory } \\
\text { Status }\end{array}$ & $\begin{array}{l}\text { Safeguards } \\
\text { and Security }\end{array}$ & $\begin{array}{l}\text { Human } \\
\text { Capital }\end{array}$ \\
\hline
\end{tabular}


Table 3-11. (continued).

Applicable Assets for

High-Temperature Reactor R\&D

Facility

Class

$(1,2,3)$
Assessment of Attributes

\section{Specialized Laboratory Facilities}

\begin{tabular}{|c|c|c|c|c|c|c|c|}
\hline $\begin{array}{l}\text { Natural Convection Shutdown Heat } \\
\text { Removal Test Facility, Argonne } \\
\text { National Laboratory }\end{array}$ & 2 & Condition & Capability & Availability & $\begin{array}{l}\text { Regulatory } \\
\text { Status }\end{array}$ & $\begin{array}{l}\text { Safeguards } \\
\text { and Security }\end{array}$ & $\begin{array}{l}\text { Human } \\
\text { Capital }\end{array}$ \\
\hline $\begin{array}{l}\text { Materials Testing Facility, Argonne } \\
\text { National Laboratory }\end{array}$ & 2 & Condition & Capability & Availability & $\begin{array}{l}\text { Regulatory } \\
\text { Status }\end{array}$ & $\begin{array}{l}\text { Safeguards } \\
\text { and Security }\end{array}$ & $\begin{array}{l}\text { Human } \\
\text { Capital }\end{array}$ \\
\hline $\begin{array}{l}\text { Materials Testing Facilities (metals, } \\
\text { ceramics, and composites), Idaho } \\
\text { National Laboratory }\end{array}$ & 2 & Condition & Capability & Availability & $\begin{array}{l}\text { Regulatory } \\
\text { Status }\end{array}$ & $\begin{array}{l}\text { Safeguards } \\
\text { and Security }\end{array}$ & $\begin{array}{l}\text { Human } \\
\text { Capital }\end{array}$ \\
\hline $\begin{array}{l}\text { Safety and Tritium Applied Research } \\
\text { Facility, Idaho National Laboratory }\end{array}$ & 2 & Condition & Capability & Availability & $\begin{array}{l}\text { Regulatory } \\
\text { Status }\end{array}$ & $\begin{array}{l}\text { Safeguards } \\
\text { and Security }\end{array}$ & $\begin{array}{l}\text { Human } \\
\text { Capital }\end{array}$ \\
\hline $\begin{array}{l}\text { Low Activity Materials Development } \\
\text { and Analysis Facility, Oak Ridge } \\
\text { National Laboratory }\end{array}$ & 2 & Condition & Capability & Availability & $\begin{array}{l}\text { Regulatory } \\
\text { Status }\end{array}$ & $\begin{array}{l}\text { Safeguards } \\
\text { and Security }\end{array}$ & $\begin{array}{l}\text { Human } \\
\text { Capital }\end{array}$ \\
\hline $\begin{array}{l}\text { Materials Testing Facilities (metals, } \\
\text { ceramics, and composites), Oak } \\
\text { Ridge National Laboratory }\end{array}$ & 2 & Condition & Capability & Availability & $\begin{array}{l}\text { Regulatory } \\
\text { Status }\end{array}$ & $\begin{array}{l}\text { Safeguards } \\
\text { and Security }\end{array}$ & $\begin{array}{l}\text { Human } \\
\text { Capital }\end{array}$ \\
\hline $\begin{array}{l}\text { Material Sciences Laboratory } \\
\text { (Building 326), Pacific Northwest } \\
\text { National Laboratory }\end{array}$ & 2 & Condition & Capability & Availability & $\begin{array}{l}\text { Regulatory } \\
\text { Status }\end{array}$ & $\begin{array}{l}\text { Safeguards } \\
\text { and Security }\end{array}$ & $\begin{array}{l}\text { Human } \\
\text { Capital }\end{array}$ \\
\hline $\begin{array}{l}\text { Physical Sciences Facility (under } \\
\text { construction), Pacific Northwest } \\
\text { National Laboratory }\end{array}$ & 2 & Condition & Capability & Availability & $\begin{array}{l}\text { Regulatory } \\
\text { Status }\end{array}$ & $\begin{array}{l}\text { Safeguards } \\
\text { and Security }\end{array}$ & $\begin{array}{l}\text { Human } \\
\text { Capital }\end{array}$ \\
\hline
\end{tabular}

\subsubsection{Proposed Facilities.}

- $\quad$ Test Train Assembly Facility, Idaho National Laboratory

- $\quad$ Component Test Facility, location TBD

- $\quad$ High-Efficiency Electrical Power Conversion Facility, location TBD

- $\quad$ Process Heat Transfer/Exchanger Facility, location TBD

- Instrumentation and Control Systems Test Facility, location TBD.

\section{Summary of Reviewer Comments}

1. Add ORNL RAG laboratories, LAMDA, IMET, HFIR; INL ATR; SNL ACRR

2. There were suggestions for modifications to color ratings of specific facilities. 


\subsubsection{Sodium Fast Reactors}

It should be noted that facility attributes indicated in a red or orange color in the stoplight color-coded rating system will require a substantial or major investment to restore them to a fully functional state; however, those investments may still be cost-effective options. Table 3-12 provides a summary reminder of the rating system's assessment designators and Table 3-13 shows the facility assessments for sodium fast reactor R\&D support.

NOTE: Stoplight assessments in the following tables are preliminary and have not been validated. Parameters are assessed for an expected 20 years of future operation.

Table 3-12. Summary example of the facility stoplight color-coded rating system.

\begin{tabular}{|l|l|}
\hline Green & Mission ready \\
\hline Yellow & Ready within 2 years or less with modest investment \\
\hline Orange & Needs modification or upgrades that require significant investment \\
\hline Red & Usable only after major capital investment over an extended number of years \\
\hline
\end{tabular}

Table 3-13. Facilities assessed for sodium fast reactor research and development support.

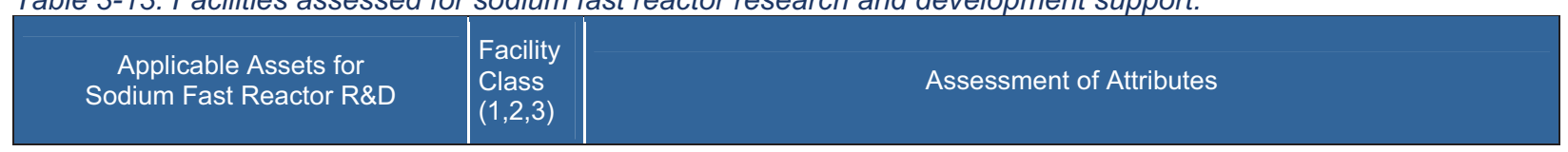

\section{Irradiation Facilities}

\begin{tabular}{|l|c|l|l|l|l|l|l|}
\hline $\begin{array}{l}\text { Fast Flux Test Facility, Richland, } \\
\text { Washington }\end{array}$ & $\mathbf{1}$ & Condition & Capability & Availability & $\begin{array}{l}\text { Regulatory } \\
\text { Status }\end{array}$ & $\begin{array}{l}\text { Safeguards } \\
\text { and Security }\end{array}$ & $\begin{array}{l}\text { Human } \\
\text { Capital }\end{array}$ \\
\hline $\begin{array}{l}\text { High Flux Isotope Reactor, Oak } \\
\text { Ridge National Laboratory }\end{array}$ & $\mathbf{1}$ & Condition & Capability & Availability & $\begin{array}{l}\text { Regulatory } \\
\text { Status }\end{array}$ & $\begin{array}{l}\text { Safeguards } \\
\text { and Security }\end{array}$ & $\begin{array}{l}\text { Human } \\
\text { Capital }\end{array}$ \\
\hline $\begin{array}{l}\text { Transient Reactor Test Facility, } \\
\text { Idaho National Laboratory }\end{array}$ & $\mathbf{1}$ & Condition & Capability & Availability & $\begin{array}{l}\text { Regulatory } \\
\text { Status }\end{array}$ & $\begin{array}{l}\text { Safeguards } \\
\text { and Security }\end{array}$ & $\begin{array}{l}\text { Human } \\
\text { Capital }\end{array}$ \\
\hline $\begin{array}{l}\text { Zero Power Physics Reactor, Idaho } \\
\text { National Laboratory }\end{array}$ & $\mathbf{1}$ & Condition & Capability & Availability & $\begin{array}{l}\text { Regulatory } \\
\text { Status }\end{array}$ & $\begin{array}{l}\text { Safeguards } \\
\text { and Security }\end{array}$ & $\begin{array}{l}\text { Human } \\
\text { Capital }\end{array}$ \\
\hline
\end{tabular}

\section{Hot Cells}

\begin{tabular}{|c|c|c|c|c|c|c|c|}
\hline $\begin{array}{l}\text { Fuel Conditioning Facility, Idaho } \\
\text { National Laboratory }\end{array}$ & 1 & Condition & Capability & Availability & $\begin{array}{l}\text { Regulatory } \\
\text { Status }\end{array}$ & $\begin{array}{l}\text { Safeguards } \\
\text { and Security }\end{array}$ & $\begin{array}{l}\text { Human } \\
\text { Capital }\end{array}$ \\
\hline $\begin{array}{l}\text { Hot Fuel Examination Facility, Idaho } \\
\text { National Laboratory }\end{array}$ & 1 & Condition & Capability & Availability & $\begin{array}{l}\text { Regulatory } \\
\text { Status }\end{array}$ & $\begin{array}{l}\text { Safeguards } \\
\text { and Security }\end{array}$ & $\begin{array}{l}\text { Human } \\
\text { Capital }\end{array}$ \\
\hline $\begin{array}{l}\text { Irradiated Fuels Exam Laboratory, } \\
\text { Oak Ridge National Laboratory }\end{array}$ & 1 & Condition & Capability & Availability & $\begin{array}{l}\text { Regulatory } \\
\text { Status }\end{array}$ & $\begin{array}{l}\text { Safeguards } \\
\text { and Security }\end{array}$ & $\begin{array}{l}\text { Human } \\
\text { Capital }\end{array}$ \\
\hline $\begin{array}{l}\text { Irradiated Materials Examination and } \\
\text { Testing Facility, Oak Ridge National } \\
\text { Laboratory }\end{array}$ & 2 & Condition & Capability & Availability & $\begin{array}{l}\text { Regulatory } \\
\text { Status }\end{array}$ & $\begin{array}{l}\text { Safeguards } \\
\text { and Security }\end{array}$ & $\begin{array}{l}\text { Human } \\
\text { Capital }\end{array}$ \\
\hline $\begin{array}{l}\text { Radiochemical Engineering } \\
\text { Development Center (REDC-7920) } \\
\text { Oak Ridge National Laboratory }\end{array}$ & 2 & Condition & Capability & Availability & $\begin{array}{l}\text { Regulatory } \\
\text { Status }\end{array}$ & $\begin{array}{l}\text { Safeguards } \\
\text { and Security }\end{array}$ & $\begin{array}{l}\text { Human } \\
\text { Capital }\end{array}$ \\
\hline
\end{tabular}


Table 3-13. (continued).

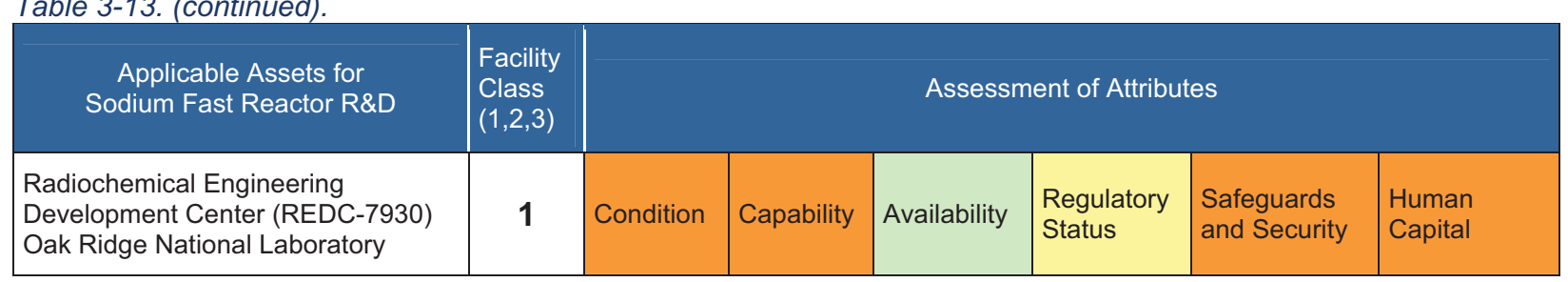

\section{Radiochemistry Laboratories}

\begin{tabular}{|l|l|l|l|l|l|l|}
\hline $\begin{array}{l}\text { Materials and Fuels Complex } \\
\text { Analytical Laboratory, Idaho National } \\
\text { Laboratory }\end{array}$ & 1 & Condition & Capability & Availability & $\begin{array}{l}\text { Regulatory } \\
\text { Status }\end{array}$ & $\begin{array}{l}\text { Safeguards } \\
\text { and Security }\end{array}$ \\
\hline
\end{tabular}

\section{Fuel Development Facilities}

\begin{tabular}{|l|c|l|l|l|l|l|l|}
\hline $\begin{array}{l}\text { Fuel Manufacturing Facility, Idaho } \\
\text { National Laboratory }\end{array}$ & $\mathbf{1}$ & Condition & Capability & Availability & $\begin{array}{l}\text { Regulatory } \\
\text { Status }\end{array}$ & $\begin{array}{l}\text { Safeguards } \\
\text { and Security }\end{array}$ & $\begin{array}{l}\text { Human } \\
\text { Capital }\end{array}$ \\
\hline $\begin{array}{l}\text { TA-55 PF-4, Los Alamos National } \\
\text { Laboratory }\end{array}$ & $\mathbf{1}$ & Condition & Capability & Availability & $\begin{array}{l}\text { Regulatory } \\
\text { Status }\end{array}$ & $\begin{array}{l}\text { Safeguards } \\
\text { and Security }\end{array}$ & $\begin{array}{l}\text { Human } \\
\text { Capital }\end{array}$ \\
\hline
\end{tabular}

\subsubsection{Proposed Facilities.}

- $\quad$ Prototype or test reactor, location TBD

- $\quad$ Materials Test Station, TA-53, (LANCE), Los Alamos National Laboratory

- $\quad$ Advanced Fuel Cycle Facility, location TBD.

3.2.6.2 Other Considered Facilities. Other facilities identified as capable of playing a limited support role (i.e., Class 3 facilities) for sodium fast reactor R\&D include the Sodium Process Facility at the Idaho National Laboratory.

\section{Summary of Reviewer Comments}

1. Add SNL ACRR.

2. There were suggestions for modifications to color ratings of specific facilities.

\subsubsection{Space and Defense Power Systems}

It should be noted that facility attributes indicated in a red or orange color in the stoplight color-coded rating system will require a substantial or major investment to restore them to a fully functional state; however, those investments may still be cost-effective options. Table 3-14 provides a summary reminder of the rating system's assessment designators and Table 3-15 shows the facility assessments for space and defense power systems R\&D support.

NOTE: Stoplight assessments in the following tables are preliminary and have not been validated. Parameters are assessed for an expected 20 years of future operation. 
Table 3-14. Summary example of the facility stoplight color-coded rating system.

\begin{tabular}{|l|l|}
\hline Green & Mission ready \\
\hline Yellow & Ready within 2 years or less with modest investment \\
\hline Orange & Needs modification or upgrades that require significant investment \\
\hline Red & Usable only after major capital investment over an extended number of years \\
\hline
\end{tabular}

Table 3-15. Facilities assessed for space and defense power systems research and development support.

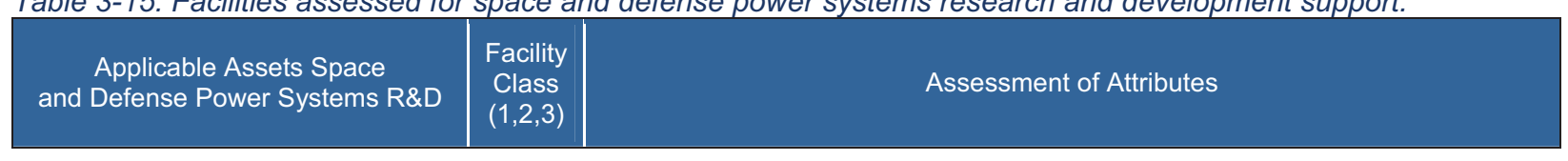

\section{Irradiation Facilities}

\begin{tabular}{|l|c|l|l|l|l|l|l|}
\hline $\begin{array}{l}\text { Advanced Test Reactor, Idaho } \\
\text { National Laboratory }\end{array}$ & $\mathbf{1}$ & Condition & Capability & Availability & $\begin{array}{l}\text { Regulatory } \\
\text { Status }\end{array}$ & $\begin{array}{l}\text { Safeguards } \\
\text { and Security }\end{array}$ & $\begin{array}{l}\text { Human } \\
\text { Capital }\end{array}$ \\
\hline $\begin{array}{l}\text { High Flux Isotope Reactor, Oak } \\
\text { Ridge National Laboratory }\end{array}$ & $\mathbf{1}$ & Condition & Capability & Availability & $\begin{array}{l}\text { Regulatory } \\
\text { Status }\end{array}$ & $\begin{array}{l}\text { Safeguards } \\
\text { and Security }\end{array}$ & $\begin{array}{l}\text { Human } \\
\text { Capital }\end{array}$ \\
\hline
\end{tabular}

\section{Hot Cells}

\begin{tabular}{|c|c|c|c|c|c|c|c|}
\hline $\begin{array}{l}\text { Fuels and Materials Examination } \\
\text { Facility, Pacific Northwest National } \\
\text { Laboratory }\end{array}$ & 1 & Condition & Capability & Availability & $\begin{array}{l}\text { Regulatory } \\
\text { Status }\end{array}$ & $\begin{array}{l}\text { Safeguards } \\
\text { and Security }\end{array}$ & $\begin{array}{l}\text { Human } \\
\text { Capital }\end{array}$ \\
\hline $\begin{array}{l}\text { Fuels Processing Restoration } \\
\text { Facility, CPP-691, Idaho National } \\
\text { Laboratory }\end{array}$ & 1 & Condition & Capability & Availability & $\begin{array}{l}\text { Regulatory } \\
\text { Status }\end{array}$ & $\begin{array}{l}\text { Safeguards } \\
\text { and Security }\end{array}$ & $\begin{array}{l}\text { Human } \\
\text { Capital }\end{array}$ \\
\hline $\begin{array}{l}\text { H-Canyon, Savannah River National } \\
\text { Laboratory }\end{array}$ & 1 & Condition & Capability & Availability & $\begin{array}{l}\text { Regulatory } \\
\text { Status }\end{array}$ & $\begin{array}{l}\text { Safeguards } \\
\text { and Security }\end{array}$ & $\begin{array}{l}\text { Human } \\
\text { Capital }\end{array}$ \\
\hline $\begin{array}{l}\text { Radiochemical Engineering } \\
\text { Development Center (Building 7920), } \\
\text { Oak Ridge National Laboratory }\end{array}$ & 1 & Condition & Capability & Availability & $\begin{array}{l}\text { Regulatory } \\
\text { Status }\end{array}$ & $\begin{array}{l}\text { Safeguards } \\
\text { and Security }\end{array}$ & $\begin{array}{l}\text { Human } \\
\text { Capital }\end{array}$ \\
\hline \multicolumn{8}{|l|}{ Radiochemistry Laboratories } \\
\hline $\begin{array}{l}\text { Remote Analytical Laboratory, } \\
\text { CPP-684, Idaho National Laboratory }\end{array}$ & 1 & Condition & Capability & Availability & $\begin{array}{l}\text { Regulatory } \\
\text { Status }\end{array}$ & $\begin{array}{l}\text { Safeguards } \\
\text { and Security }\end{array}$ & $\begin{array}{l}\text { Human } \\
\text { Capital }\end{array}$ \\
\hline
\end{tabular}

\section{Fuel Development Facilities}

\begin{tabular}{|l|c|l|l|l|l|l|l|}
\hline $\begin{array}{l}\text { Fuel Manufacturing Facility, Idaho } \\
\text { National Laboratory }\end{array}$ & $\mathbf{1}$ & Condition & Capability & Availability & $\begin{array}{l}\text { Regulatory } \\
\text { Status }\end{array}$ & $\begin{array}{l}\text { Safeguards } \\
\text { and Security }\end{array}$ & $\begin{array}{l}\text { Human } \\
\text { Capital }\end{array}$ \\
\hline $\begin{array}{l}\text { TA-55/PF-4, Los Alamos National } \\
\text { Laboratory }\end{array}$ & $\mathbf{1}$ & Condition & Capability & Availability & $\begin{array}{l}\text { Regulatory } \\
\text { Status }\end{array}$ & $\begin{array}{l}\text { Safeguards } \\
\text { and Security }\end{array}$ & $\begin{array}{l}\text { Human } \\
\text { Capital }\end{array}$ \\
\hline
\end{tabular}

\section{Specialized Laboratory Facilities}

\begin{tabular}{|l|l|l|l|l|l|l|l|}
\hline $\begin{array}{l}\text { Space and Security Power Systems } \\
\text { Facility, Idaho National Laboratory }\end{array}$ & $\mathbf{1}$ & Condition & Capability & Availability & $\begin{array}{l}\text { Regulatory } \\
\text { Status }\end{array}$ & $\begin{array}{l}\text { Safeguards } \\
\text { and Security }\end{array}$ & $\begin{array}{l}\text { Human } \\
\text { Capital }\end{array}$ \\
\hline
\end{tabular}




\subsubsection{Proposed Facilities.}

- $\quad$ New Training, Research, and Isotope Reactor, location TBD

- $\quad$ Pu-238 Consolidation, Idaho National Laboratory

- $\quad$ Zero Power Physics Reactor plus new/upgrades, Idaho National Laboratory.

\section{Summary of Reviewer Comments}

1. Add ORNL facilities REDC-7920, 7930, and the RAG Laboratories; add the safety testing capabilities at LANL.

2. Make the introduction clear that only radioisotope power systems and not space reactors and included. 


\subsection{Domestic Facility Descriptions}

\subsubsection{Department of Energy National Laboratories}

\section{Advanced Test Reactor (ATR)}

Location: Idaho National Laboratory

Currently Supporting: Multiple programs

Status: Fully operational

Remarks: Life-extension upgrades are in progress

\begin{tabular}{|l|l|l|l|}
\hline Potential Program R\&D Applications are Shaded \\
\hline Light Water Reactors & $\begin{array}{l}\text { Irradiated Fuel } \\
\text { Separations }\end{array}$ & $\begin{array}{l}\text { Advanced Fuel } \\
\text { Development }\end{array}$ & Space Power Systems \\
\hline $\begin{array}{l}\text { High-Temperature } \\
\text { Reactor }\end{array}$ & Fast Spectrum Reactor & $\begin{array}{l}\text { Safeguards and } \\
\text { Security }\end{array}$ & $\begin{array}{l}\text { Modeling and } \\
\text { Simulation }\end{array}$ \\
\hline
\end{tabular}

ATR has been operating continuously since 1967; however, because its internal components are periodically changed out, it remains a valuable research and test machine capable of decades of more service. It was established as a National Scientific User Facility by DOE in 2007.

ATR is a low-temperature and pressure water-cooled reactor designed to study the effects of intense radiation on reactor materials and fuels. In addition, ATR irradiates targets to produce valuable isotopes for medical, industrial, and research applications.

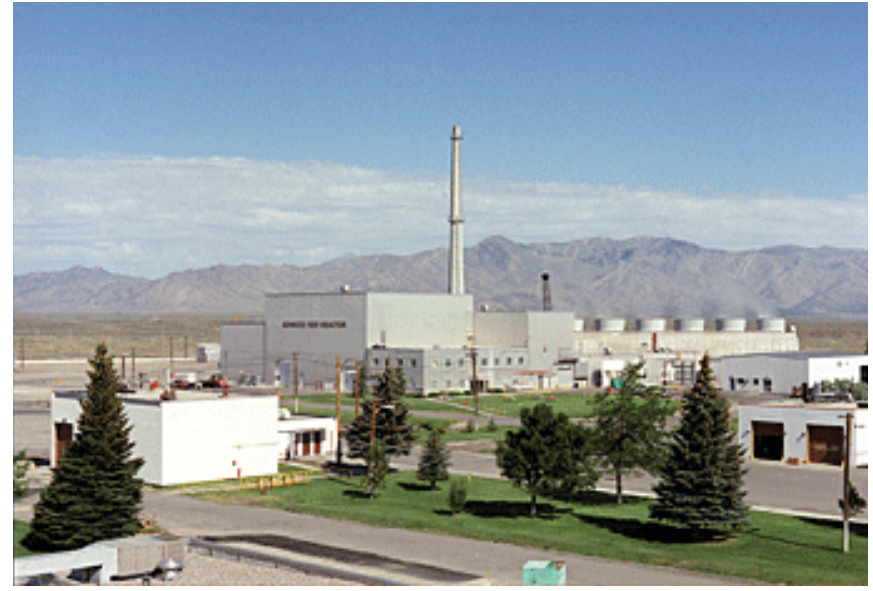

The Advanced Test Reactor at the Idaho National Laboratory
The ATR core is divided into five different operating lobes: the four corner lobes and the center lobe. Each lobe of the reactor may be operated at a different power level (within specific limitations) during each reactor cycle. Three major types of irradiation testing are employed: 1) static sealed-capsule tests with only passive instrumentation; 2) tests with active instrumentation for measurement and control of specific testing parameters; and 3 ) pressurized water loops that are connected to in-pile tubes located in the flux traps.

The ATR has a maximum power of $250 \mathrm{MW}$ and can provide maximum thermal neutron fluxes of $1 \mathrm{E} 15$ neutrons per square centimeter per second and maximum fast $(E>1.0 \mathrm{MeV})$ neutron fluxes of $5 E 14$ neutrons per square centimeter per second. These fluences combined with the 77 irradiation positions varying in diameter from $16 \mathrm{~mm}$ (0.625 in.) to $127 \mathrm{~mm}$ (5.0 in.) over an active core height of $1.2 \mathrm{~m} \mathrm{(48.0} \mathrm{in.)} \mathrm{make} \mathrm{ATR} \mathrm{a} \mathrm{very}$ versatile and unique facility. 


\section{Annular Core Research Reactor (ACRR)}

Location: Sandia National Laboratories

Currently Supporting: Multiple programs

Status: Fully operational

Remarks: Life-extension upgrades recently completed

\begin{tabular}{|l|l|l|l|}
\hline Potential Program R\&D Applications are Shaded \\
\hline Light Water Reactors & $\begin{array}{l}\text { Irradiated Fuel } \\
\text { Separations }\end{array}$ & $\begin{array}{l}\text { Advanced Fuel } \\
\text { Development }\end{array}$ & Space Power Systems \\
\hline $\begin{array}{l}\text { High-Temperature } \\
\text { Reactor }\end{array}$ & Fast Spectrum Reactor & $\begin{array}{l}\text { Safeguards and } \\
\text { Security }\end{array}$ & $\begin{array}{l}\text { Modeling and } \\
\text { Simulation }\end{array}$ \\
\hline
\end{tabular}

The Annular Core Research Reactor is a pool-type research reactor capable of pulsed operation, steadystate operation, and tailored transient rod withdrawal operation. This facility provides a large $(23-\mathrm{cm} \mathrm{dia}$.) central irradiation chamber, a neutron radiography facility, two large (38- and 51-cm dia.) interchangeable fuel ringed external cavities, and a large $(130 \times 20 \mathrm{~cm})$ rectangular unfueled external cavity.

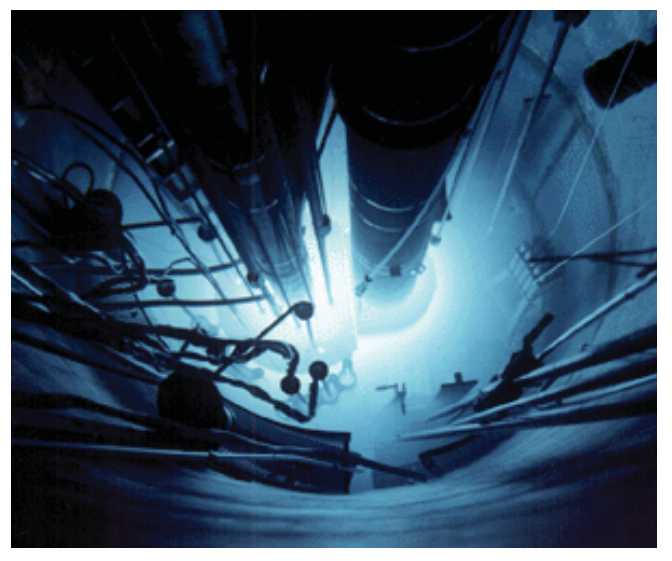

The annular-shaped core is formed by 236 fuel elements arranged in a hexagonal grid around the $23-\mathrm{cm}$ diameter, central irradiation cavity. The reactor is controlled by two fuelfollowed safety rods, three poison transient rods, and six fuelfollowed control rods. The fuel-followed rods make up part of the 236 elements for the normal core configuration.

Additionally, the external irradiation cavities of $38-$ and $51-\mathrm{cm}$ diameters are ringed with 80 and $182 \mathrm{U}-\mathrm{ZrH}$ fuel elements, respectively.

The cylindrical fuel elements contain a uniquely designed $\mathrm{BeO}-\mathrm{UO} 2$ fuel material held with niobium liners inside a stainless steel cladding. The fuel is uranium, enriched to 35 percent $\mathrm{U} 235$ with 21.5 weight percent $\mathrm{UO} 2$ and 78.5 weightpercent $\mathrm{BeO}$. The $\mathrm{BeO}$ fuel elements were designed to allow operation at fuel temperatures up to $1400^{\circ} \mathrm{C}$ in both the pulse and steady state modes.

The core is located in an open pool, $3.1 \mathrm{~m}$ in diameter and $8.5 \mathrm{~m}$ deep, which contains 16,800 gallons of deionized water. The core is cooled by natural convection. The bulk water and the cooling-tower systems are used to maintain the pool water near ambient temperature. A large high-bay area is adjacent to the pool for instrumentation pads associated with experiments.

ACRR Performance Characteristics Maximum Nominal Operating Parameters

\author{
Pulse Operation \\ Reactivity Insertion $\$ 3.00$ \\ Peak Power 30,000 MW \\ Pulse Width Programmable \\ Energy Release $300 \mathrm{MJ}$
}

\author{
Steady-State Operation \\ Reactor Power (Continuous) 2.0 MW \\ Reactor Power (Intermittent) 4.0 MW
}




\section{Chemical and Metallurgical Research Facility (Wings 5, 7, and 9)}

Location: Los Alamos National Laboratory

Currently Supporting: Multiple Programs

Status: Fully operational

\begin{tabular}{|l|l|l|l|}
\hline Potential Program R\&D Applications are Shaded \\
\hline Light Water Reactors & $\begin{array}{l}\text { Irradiated Fuel } \\
\text { Separations }\end{array}$ & $\begin{array}{l}\text { Advanced Fuel } \\
\text { Development }\end{array}$ & Space Power Systems \\
\hline $\begin{array}{l}\text { High-Temperature } \\
\text { Reactor }\end{array}$ & Fast Spectrum Reactor & $\begin{array}{l}\text { Safeguards and } \\
\text { Security }\end{array}$ & $\begin{array}{l}\text { Modeling and } \\
\text { Simulation }\end{array}$ \\
\hline
\end{tabular}

The Chemical and Metallurgical Research Facility, constructed in 1952, is a 550,000 $\mathrm{ft}^{2}$ laboratory complex with a hot cell wing (Wing 9) that was added to the facility in 1961. The facility is Security Category III, Hazard Category 2, and has six wings, each with $8,000 \mathrm{ft}^{2}$ of laboratory space. Several of the wings of the Chemical and Metallurgical Research Facility have discontinued programmatic work, but the main facility, three laboratory wings, and the hot cells are continuing to serve multiple programs. An $\$ 8 \mathrm{M}$ infrastructure upgrade project is currently being implemented to prepare the facility for demonstration of remote fabrication of advanced oxide fuels.

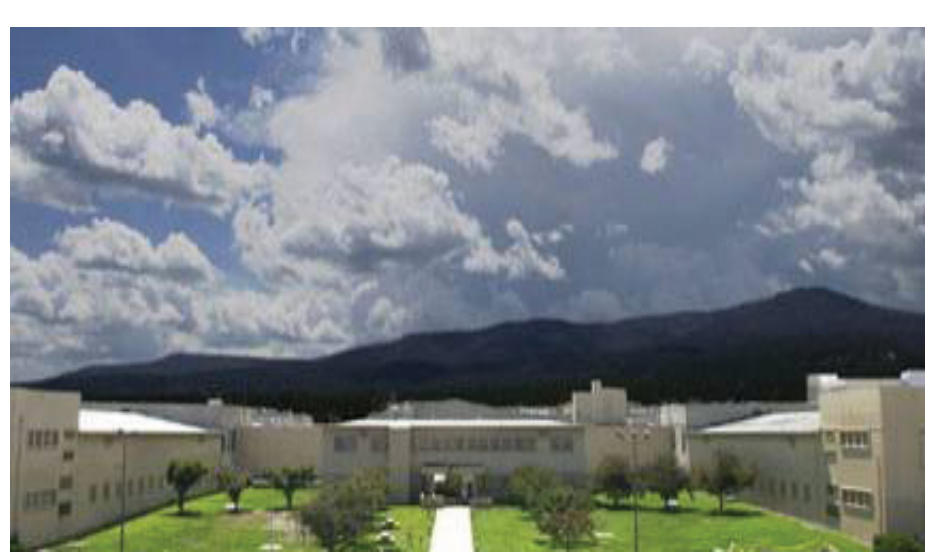

The Chemical and Metallurgical Research building at Los Alamos National Laboratory
The current authorization basis (Basis for Interim Operations) expires in 2010 and a new documented safety analysis is to be completed and implemented by 2010. Many life-extension issues will not be resolved until the documented safety analysis is completed. Critical Decision-0 has been approved for the Chemical and Metallurgical Research replacement facility and conceptual design effort is ongoing. Wing 9, however, may be modified to be a separate facility and remain in operation.

Wing 9 contains 16 hot cells $(6 \mathrm{ft} \times 6 \mathrm{ft} \times$ $11 \mathrm{ft}$ high) that are arranged in eight pairs of two. They can be configured as 16 separate hot cells in two banks of eight on each side

of a central corridor. Cell access is by crane or by a cart on rails. Contamination control for processes involving dispersible radioactive materials and inert atmosphere operation, if needed, is accomplished by use of in-cell sealed alpha boxes. Use of alpha boxes has kept the cells relatively clean of contamination, and man-entries are possible in most cells. Wings 5 and 7 provide analytical chemistry support, with certification as a NIST Reference Laboratory and metallurgical study capability for small samples of uranium, plutonium, and other special nuclear materials. 


\section{Electron Microscopy Laboratory}

Location: Idaho National Laboratory

Currently Supporting: Multiple programs

Status: Operating

\begin{tabular}{|l|l|l|l|}
\hline Potential Program R\&D Applications are Shaded \\
\hline Light Water Reactors & $\begin{array}{l}\text { Irradiated Fuel } \\
\text { Separations }\end{array}$ & $\begin{array}{l}\text { Advanced Fuel } \\
\text { Development }\end{array}$ & Space Power Systems \\
\hline $\begin{array}{l}\text { High-Temperature } \\
\text { Reactor }\end{array}$ & Fast Spectrum Reactor & $\begin{array}{l}\text { Safeguards and } \\
\text { Security }\end{array}$ & $\begin{array}{l}\text { Modeling and } \\
\text { Simulation }\end{array}$ \\
\hline
\end{tabular}
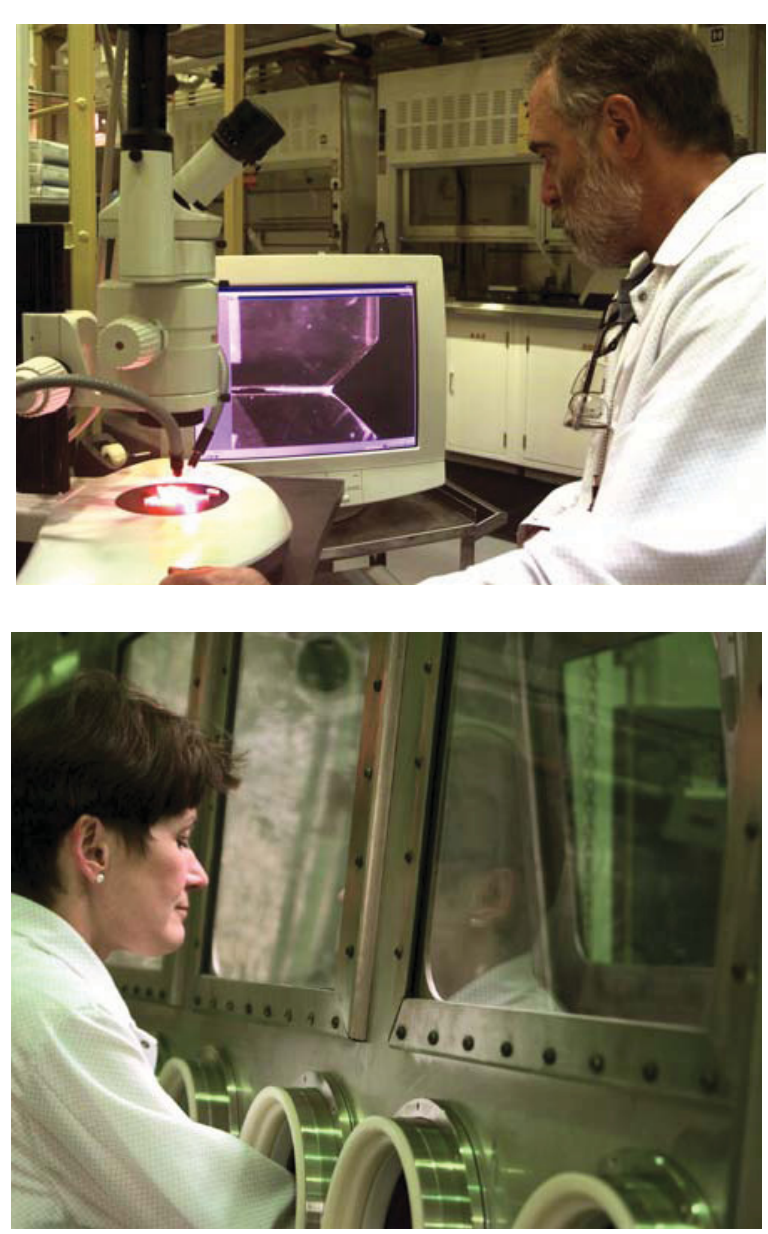

Examination and sample preparation in the Electron Microscopy Laboratory at the Idaho National Laboratory
The Electron Microscopy Laboratory is a $40 \mathrm{ft} \times 40 \mathrm{ft} \times 26 \mathrm{ft}$-high cinder block room, located on the north side of the Zero Power Physics Reactor at the Materials and Fuels Complex of the Idaho National Laboratory.

The Electron Microscopy Laboratory contains a glovebox, fume hoods, and a variety of specimen preparation equipment for preparing radioactive metals, ceramics, small quantities of fissionable material for microscopic examination, and standard PCs for easy data and image acquisition, storage, and analysis. Examination capabilities include transmission electron microscopy with x-ray spectroscopy and electron diffraction; scanning electron microscopy with $x$-ray spectroscopy and electron back scatter diffraction; and optical microscopy with microhardness measurement. The three primary instruments in the Electron Microscopy Laboratory are 1) a JEOL 2010 scanning transmission electron microscope capable of operating at $200 \mathrm{kV}$ with magnifications from 2,000 X to $1,500,000 \mathrm{X} ; 2$ ) a JEOL JSM-7000f scanning electron microscope capable of operating at $30 \mathrm{kV}$ with magnifications from $15 \mathrm{X}$ to $500,000 \mathrm{X}$; and 3) a Zeiss DSM 960 scanning electron microscope capable of operating at $30 \mathrm{kV}$ with magnifications from $6 \mathrm{X}$ to 200,000 X. Each electron microscope is equipped with $\mathrm{x}$-ray spectrometers to gather elemental and crystallographic sample information. 


\section{Fuels and Applied Science Building}

Location: Idaho National Laboratory

Currently Supporting: Multiple programs

Status: Operating

\begin{tabular}{|l|l|l|l|}
\hline Potential Program R\&D Applications are Shaded \\
\hline Light Water Reactors & $\begin{array}{l}\text { Irradiated Fuel } \\
\text { Separations }\end{array}$ & $\begin{array}{l}\text { Advanced Fuel } \\
\text { Development }\end{array}$ & Space Power Systems \\
\hline $\begin{array}{l}\text { High-Temperature } \\
\text { Reactor }\end{array}$ & Fast Spectrum Reactor & $\begin{array}{l}\text { Safeguards and } \\
\text { Security }\end{array}$ & $\begin{array}{l}\text { Modeling and } \\
\text { Simulation }\end{array}$ \\
\hline
\end{tabular}

The Fuels and Applied Science Building is a 5,000 $\mathrm{ft}^{2}$, multipurpose laboratory facility dedicated to fuel development, irradiation test fabrication, and materials research. It has both radiological and non-radiological areas that improve the efficiency for programs by streamlining operational requirements.

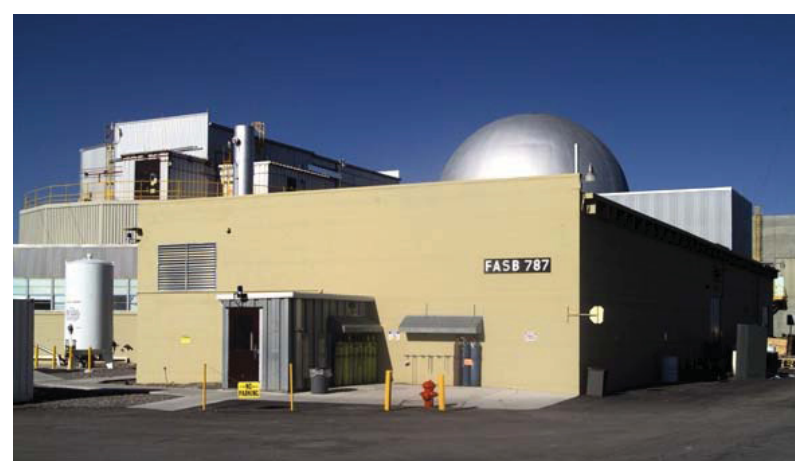

Fuels and Applied Science Building at the Idaho National Laboratory
Three glovebox installations are available. All are negative pressure, inert atmosphere (argon) gloveboxes. Various standard and specialized materials preparation equipment are available, including fume hoods (radiological and non-radiological), furnaces (resistance and inductively heated), balances, optical microscopes, uniaxial presses, and a hot isostatic press.

Fuel fabrication equipment includes mills, lathes, shears, roller presses, arc melters, arc welders, and an ultrasonic testing station. A small prototyping laboratory is available for modifying or building specialized equipment and devices. 


\section{Flourinel Dissolution Process and Fuel Storage Facility (FAST)}

Location: Idaho National Laboratory

Currently Supporting: Fuel storage

Status: Basins are active for fuel storage. Process cell is in standby.

Remarks: Facility will need a comprehensive restart effort with modifications as necessary to fit a new program

Potential Program R\&D Applications are Shaded

\begin{tabular}{|l|l|l|l|}
\hline Light Water Reactors & $\begin{array}{l}\text { Irradiated Fuel } \\
\text { Separations }\end{array}$ & $\begin{array}{l}\text { Advanced Fuel } \\
\text { Development }\end{array}$ & Space Power Systems \\
\hline $\begin{array}{l}\text { High-Temperature } \\
\text { Reactor }\end{array}$ & Fast Spectrum Reactor & $\begin{array}{l}\text { Safeguards and } \\
\text { Security }\end{array}$ & $\begin{array}{l}\text { Modeling and } \\
\text { Simulation }\end{array}$ \\
\hline
\end{tabular}

The FAST facility was constructed in 1984 to receive and store various types and sizes of fuels and to perform the head-end dissolution step in the reprocessing of zirconium-based fuel. Operations were conducted from 1985 to 1988. The facility was placed in standby in 1992.

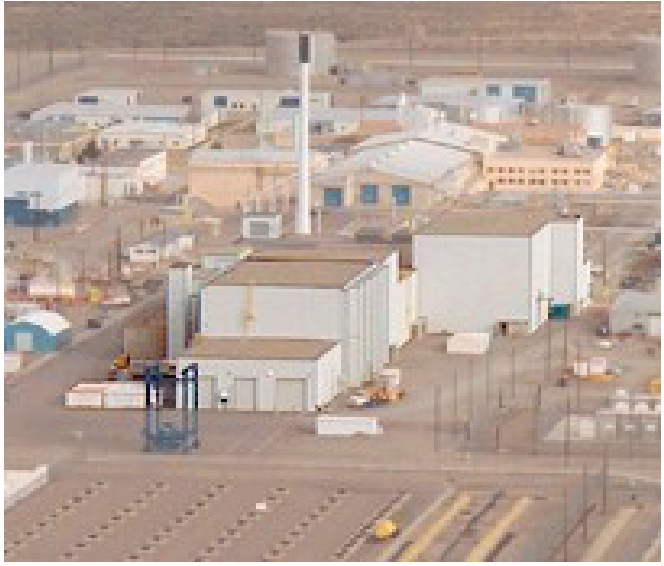

Flourinel Dissolution Process and Fuel Storage Facility facility at the Idaho National Laboratory
The FAST facility consists of the Fuel Storage Area and the Fluorinel Dissolution Process Area. The Fuel Storage Area portion of the FAST facility includes six water storage basins, two of which are deep enough for commercial LWR fuel assemblies. A cart and inclined plane allows fuel to be transferred from the basins directly into the fuel dissolution process cell. However, a cart modification would be needed to transfer commercial length assemblies into the fuel dissolution process cell. Cask-handling capabilities accommodate commercial fuel transfer casks. Fuel receipt and storage at the Fuel Storage Area is continuing until a decision is made regarding the ultimate disposition of the fuel or until alternative fuel storage options, such as dry storage, are selected and implemented

The Fluorinel Dissolution Process Area cell is a $20 \times 100 \times 50 \mathrm{ft}$, stainless steel lined, air atmosphere cell with 5 -ft thick reinforced concrete walls. It currently houses a three-train dissolver system. Three small cells for sampling, waste load out, and neutron interrogation support for Fluorinel Dissolution Process Area cell operation. A pneumatic transfer system connects the sample cell with the Remote Analytical Laboratory. 


\section{Fuel Conditioning Facility (FCF)}

Location: Idaho National Laboratory

Currently Supporting: Electrochemical processing

Status: Fully operational, refurbishment completed in 1996

Remarks: Safety analysis update is in progress

\begin{tabular}{|l|l|l|l|}
\hline Potential Program R\&D Applications are Shaded \\
\hline Light Water Reactors & $\begin{array}{l}\text { Irradiated Fuel } \\
\text { Separations }\end{array}$ & $\begin{array}{l}\text { Advanced Fuel } \\
\text { Development }\end{array}$ & Space Power Systems \\
\hline $\begin{array}{l}\text { High-Temperature } \\
\text { Reactor }\end{array}$ & Fast Spectrum Reactor & $\begin{array}{l}\text { Safeguards and } \\
\text { Security }\end{array}$ & $\begin{array}{l}\text { Modeling and } \\
\text { Simulation }\end{array}$ \\
\hline
\end{tabular}

FCF was originally constructed in the early 1960s to demonstrate recycling of EBR-II metal fuel. Major modifications were completed and new process equipment was added in the 1990s for the Integral Fast Reactor program.

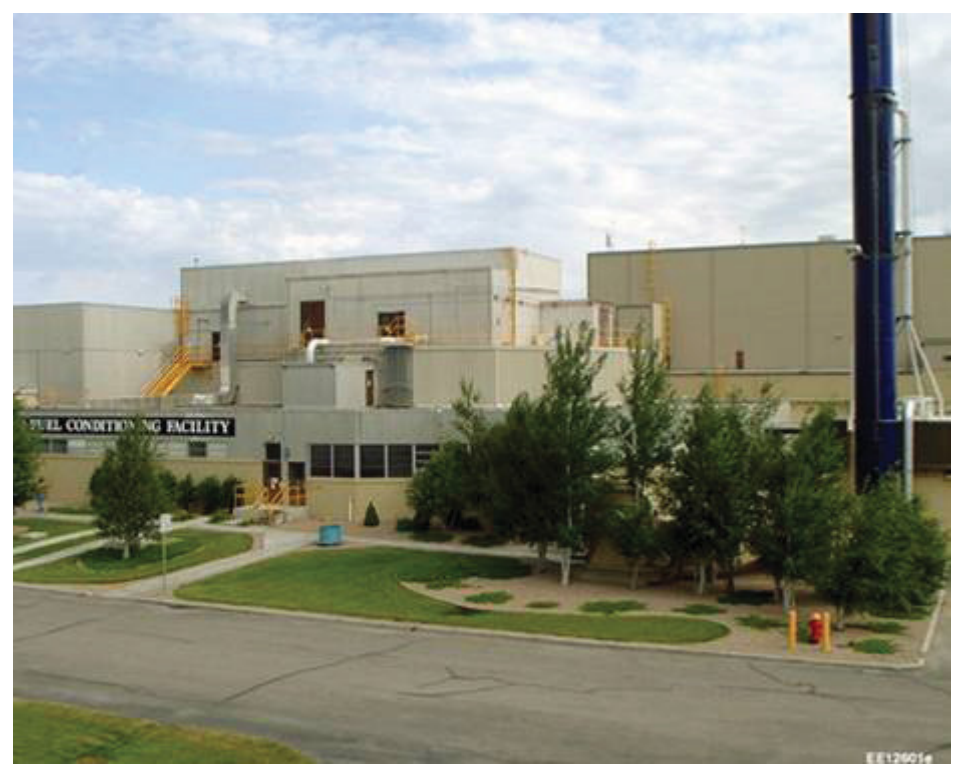

Fuel Conditioning Facility at the Idaho National Laboratory
FCF consists of two hot cells: one having an air atmosphere and the other having an inert argon gas atmosphere, which enables work with both chemically reactive materials and radioactive nuclear materials. The rectangular air cell is $52 \mathrm{ft}$ long by $25 \mathrm{ft}$ wide and $22 \mathrm{ft}$ high with nine operating stations. The air cell is used for handling, storage, and assembly/ disassembly of components. A pneumatic transfer system connects the air cell with the Materials and Fuels Complex Analytical Laboratory hot cells for rapid transfer of radioactive samples for analysis. The argon cell is a much larger "doughnut" shaped hot cell at $62 \mathrm{ft}$ in diameter by $22 \mathrm{ft}$ high with 15 operating stations. The argon atmosphere is maintained at less than 100 ppm oxygen. Shielding walls are 5-ft thick, high-density concrete. Personnel can work from the outside corridor around the hot cell and monitor in-cell activities from an inner shielded space in the center of the hot cell. All equipment in the cells can be repaired remotely using externally controlled manipulators and cranes. Currently, the facility is used to perform research and pilot-scale demonstration of the electrorefining process for irradiated, sodium-bonded, metal fuel.

In addition to the hot cells, the facility contains a mockup area where new equipment can be qualified and tested for remote operations and maintenance prior to installation in the FCF or the nearby Hot Fuel Examination Facility (HFEF) cells. Also, there is a spray chamber, special glove boxes, and a suited entry repair area (located in the basement) where contaminated equipment can be decontaminated and repaired. 


\section{Fast Flux Test Facility (FFTF)}

Location: DOE Hanford Site, Washington

\section{Currently Supporting: None}

Status: Shutdown, fuel and sodium coolant removed, listed for decommissioning and demolition

Remarks: Restart will require a major effort to repair and re-qualify systems and reload the core

\begin{tabular}{|l|l|l|l|}
\hline Potential Program R\&D Applications are Shaded \\
\hline Light Water Reactors & $\begin{array}{l}\text { Irradiated Fuel } \\
\text { Separations }\end{array}$ & $\begin{array}{l}\text { Advanced Fuel } \\
\text { Development }\end{array}$ & Space Power Systems \\
\hline $\begin{array}{l}\text { High-Temperature } \\
\text { Reactor }\end{array}$ & Fast Spectrum Reactor & $\begin{array}{l}\text { Safeguards and } \\
\text { Security }\end{array}$ & $\begin{array}{l}\text { Modeling and } \\
\text { Simulation }\end{array}$ \\
\hline
\end{tabular}

FFTF is a 400 megawatt thermal, loop-type, sodium-cooled, fast spectrum reactor located on the Hanford Site. Heat was removed from the reactor by circulating liquid sodium through three primary heat transport system loops, connected to secondary heat transport system loops. The secondary heat transport system loops transferred the heat to the atmosphere through air-cooled, dump heat exchangers.

The FFTF complex includes the reactor, heat removal equipment, and structures; containment; fuel handling; a hot cell within the containment for sodium removal and initial core component examination, instrumentation, and control; a separate fuel storage building; utilities; and other essential services.

Construction of the FFTF was completed in 1978, and the first reaction took place in 1980. From April 1982 to April 1992, the FFTF operated successfully as a national research facility to test advanced

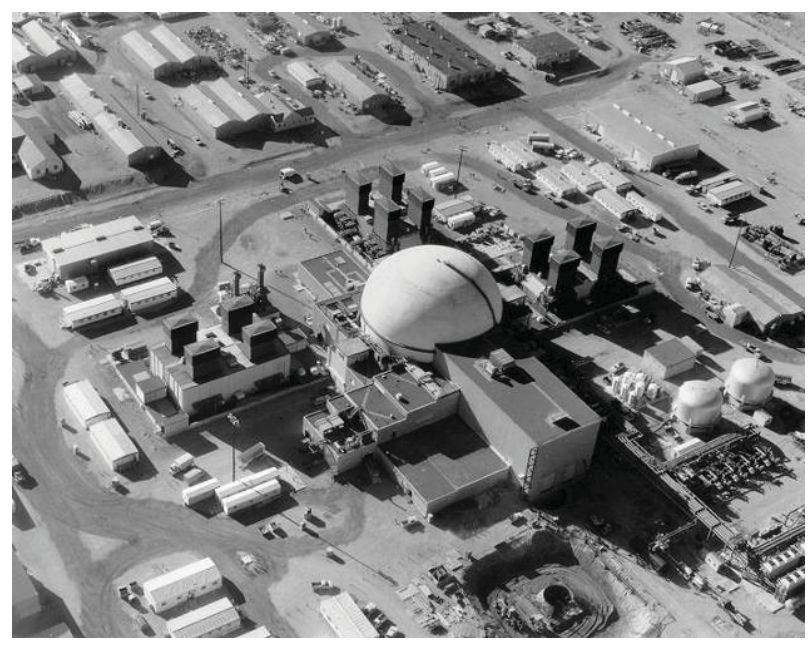

The Fast Flux Test Facility at the Department of Energy Hanford Site nuclear fuels, materials, components, nuclear power plant operations and maintenance protocols, and reactor safety designs. During this time, the FFTF also produced a wide variety of medical and industrial isotopes, made tritium for the U.S. fusion research program, and conducted cooperative international research work.

After 10 years of operation, FFTF was shut down in 1992. Although there have been several deactivation delays, potential mission studies, and legal actions, the fuel and coolant have been removed, the core support basket has been drilled to drain the remaining sodium coolant, and completion of the deactivation phase of the FFTF Closure Project continues. 


\section{Fuels and Materials Examination Facility}

Location: DOE Hanford Site

Currently Supporting: None

Status: Construction incomplete/never operated

Remarks: Programmatic use will require completion of construction with modification, as necessary, and provision of process and support equipment

Potential Program R\&D Applications are Shaded

\begin{tabular}{|l|l|l|l|}
\hline Light Water Reactors & $\begin{array}{l}\text { Irradiated Fuel } \\
\text { Separations }\end{array}$ & $\begin{array}{l}\text { Advanced Fuel } \\
\text { Development }\end{array}$ & Space Power Systems \\
\hline $\begin{array}{l}\text { High-Temperature } \\
\text { Reactor }\end{array}$ & Fast Spectrum Reactor & $\begin{array}{l}\text { Safeguards and } \\
\text { Security }\end{array}$ & $\begin{array}{l}\text { Modeling and } \\
\text { Simulation }\end{array}$ \\
\hline
\end{tabular}

The Fuels and Materials Examination Facility was designed to examine irradiated fuels and materials experiments from the FFTF and to manufacture mixed oxide fuels. Capability to include aqueous reprocessing equipment also was included. It was constructed during the early 1980s, but was never activated and operated.

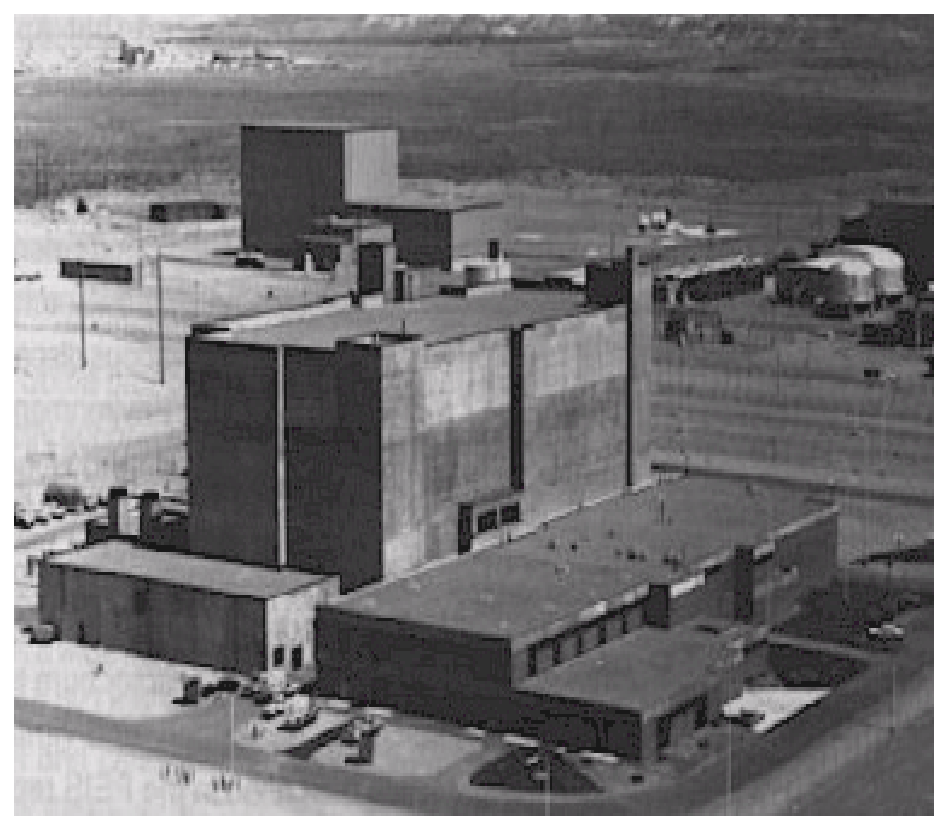

The Fuels and Materials Examination Facility at the Department of Energy Hanford Site 400 Area
The Fuels and Materials Examination Facility is a poured concrete building, $175 \mathrm{ft}$ by $270 \mathrm{ft}$ by $98 \mathrm{ft}$ high. The building also extends $35 \mathrm{ft}$ below grade and has a total of about $200,000 \mathrm{ft}^{2}$ of operations space. It is divided into six operating floors with an attached mechanical equipment wing and an entry wing. The facility was designed for both truck and rail access. A 75-ton lift crane system and a below grade tunnel for transfer of casks are present. A series of large work spaces set up with high-efficiency particulate air exhaust are built into the facility at various levels around the main cell blocks. These could be used for process support activities or analyses. Outside the work spaces are additional spaces that are well suited for personnel offices.

The largest cell is $50 \mathrm{ft}$ high with about $4,000 \mathrm{ft}^{2}$ of floor space and an adjacent maintenance cell; the upper process cell with adjacent transfer cell is $24 \mathrm{ft}$ high with about 2,300 $\mathrm{ft}^{2}$ of floor space; and the 14 process support cells are $14 \mathrm{ft}$ high with either $42 \mathrm{ft}^{2}$ or $78 \mathrm{ft}^{2}$ of floor space. All these cells are capable of being operated with an inert atmosphere. Also, there is a cell designed for a TRIGA reactor and a production scale automated MOX fuel fabrication line is installed in a glovebox on the upper level. The hot cells do not currently have windows or manipulators. A shielded and remotely operated pick and place spent nuclear material storage vault was built into the facility. 


\section{Fuel Manufacturing Facility}

Location: Idaho National Laboratory

Currently Supporting: Advanced Fuel Cycle Initiative

Status: Fully operational

Remarks: Safety analysis update is in progress

\begin{tabular}{|l|l|l|l|}
\hline Potential Program R\&D Applications are Shaded \\
\hline Light Water Reactors & $\begin{array}{l}\text { Irradiated Fuel } \\
\text { Separations }\end{array}$ & $\begin{array}{l}\text { Advanced Fuel } \\
\text { Development }\end{array}$ & Space Power Systems \\
\hline $\begin{array}{l}\text { High-Temperature } \\
\text { Reactor }\end{array}$ & Fast Spectrum Reactor & $\begin{array}{l}\text { Safeguards and } \\
\text { Security }\end{array}$ & $\begin{array}{l}\text { Modeling and } \\
\text { Simulation }\end{array}$ \\
\hline
\end{tabular}

The Fuel Manufacturing Facility began operations in 1986 to perform fuel development work and to manufacture fuel for the EBR-II reactor.

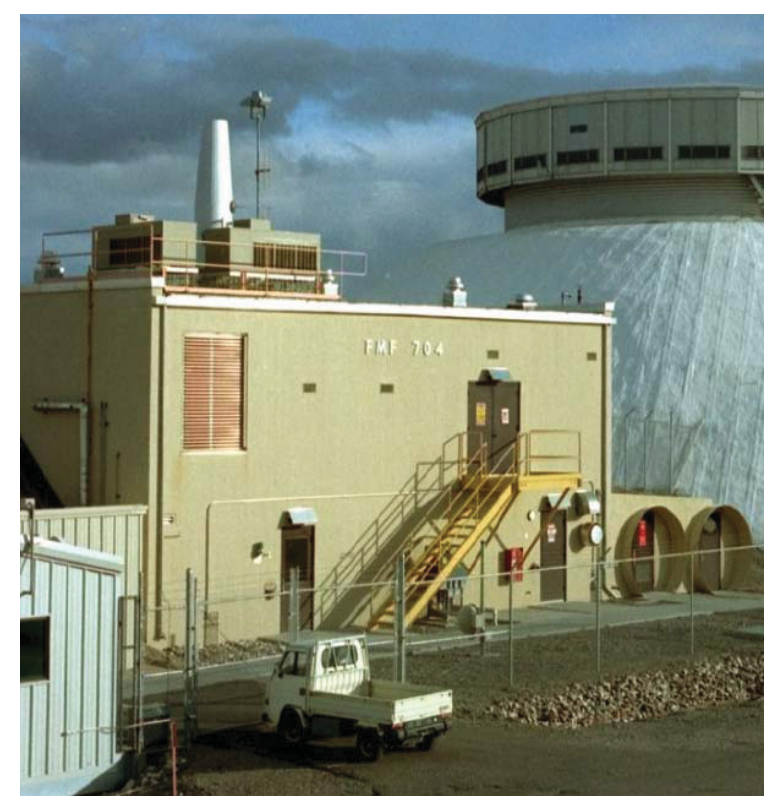

Fuel Manufacturing Facility at the Idaho National Laboratory
This seismically and security "hardened" facility is approximately $100 \mathrm{ft}$ by $50 \mathrm{ft}$ and located within the Materials and Fuels Complex. It is surrounded by a perimeter intrusion, detection, and assessment system.

Since the EBR-II reactor was shut down in 1994, the facility has continued to support new reactor fuel development work and general fissionable material handling, processing, and storage activities in the facility's numerous gloveboxes. Installation of a new inert atmosphere glovebox "train" specifically for Advanced Fuel Cycle Initiative project fuel fabrication was completed recently. 


\section{Fuel Processing Facility}

Location: Idaho National Laboratory

\section{Currently Supporting: Storage}

Status: Construction not completed; facility was never activated

Remarks: Programmatic use will require completion of construction with modification, as necessary, and provision of process and support equipment

Potential Program R\&D Applications are Shaded

\begin{tabular}{|l|l|l|l|}
\hline Light Water Reactors & $\begin{array}{l}\text { Irradiated Fuel } \\
\text { Separations }\end{array}$ & $\begin{array}{l}\text { Advanced Fuel } \\
\text { Development }\end{array}$ & Space Power Systems \\
\hline $\begin{array}{l}\text { High-Temperature } \\
\text { Reactor }\end{array}$ & Fast Spectrum Reactor & $\begin{array}{l}\text { Safeguards and } \\
\text { Security }\end{array}$ & $\begin{array}{l}\text { Modeling and } \\
\text { Simulation }\end{array}$ \\
\hline
\end{tabular}

The Fuel Processing Facility, also known as the Fuel Processing Restoration Project, was designed to process spent fuel in pulsed columns and recover enriched uranium as an oxide product. Although intended to recover high-enriched uranium, alpha control features were designed into the facility because of the potential for plutonium contamination.

The Fuel Processing Facility construction was halted in 1992 after enclosure of the structure was completed. Minimal heating and ventilation was installed for safety and investment protection. Most of the process equipment and balance of plant items (e.g., electrical, heating and ventilation, shielding windows, and manipulators control room and offices) were not purchased, installed, or completed. The facility is currently used as a storage warehouse.

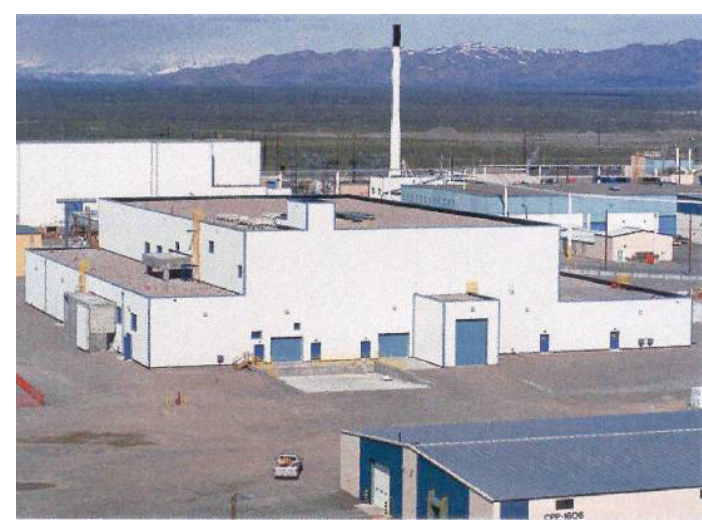

Fuel Processing Facility at the Idaho National Laboratory
The Fuel Processing Facility contains nine shielded, air atmosphere process cells, each approximately $20 \mathrm{ft}$ wide $\mathrm{x}$ $40 \mathrm{ft}$ long $x 40 \mathrm{ft}$ high. These cells are located below grade and aligned next to a $200 \mathrm{ft}$ long $\times 15 \mathrm{ft}$ wide $\times 45 \mathrm{ft}$ high "mini-canyon" pump and valve corridor.

Remotely controlled sampling cells are included to support all of the process functions. The pump and valve corridor is stainless steel lined and equipment within it can be remotely maintained. Three levels of operating corridors surround the process cells. 


\section{H-Canyon}

Location: Savannah River Site

Currently Supporting: High-enriched uranium blend-down

Status: Operational

Remarks: R\&D program use will require equipment removal and disposal, facility modifications as necessary, and provision of new process equipment

\begin{tabular}{|l|l|l|l|}
\hline Potential Program R\&D Applications are Shaded \\
\hline Light Water Reactors & $\begin{array}{l}\text { Irradiated Fuel } \\
\text { Separations }\end{array}$ & $\begin{array}{l}\text { Advanced Fuel } \\
\text { Development }\end{array}$ & Space Power Systems \\
\hline $\begin{array}{l}\text { High-Temperature } \\
\text { Reactor }\end{array}$ & Fast Spectrum Reactor & $\begin{array}{l}\text { Safeguards and } \\
\text { Security }\end{array}$ & $\begin{array}{l}\text { Modeling and } \\
\text { Simulation }\end{array}$ \\
\hline
\end{tabular}

The Savannah River $\mathrm{H}$-Canyon is a very large facility constructed over 50 years ago to recover plutonium from natural uranium targets by aqueous reprocessing methods. It was designed for process flexibility, and in the late 1950s, it was converted to recycle Savannah River Site highly enriched uranium reactor fuel. In the 1960s the process was modified to recover neptunium and a capability was added to recover and purify plutonium-238. It is now being used to blend-down highly enriched uranium into low-enriched uranium suitable for commercial reactor fuel. Most of the control systems are, however, the original 1950s technology.

The main process vessels are located in long, air atmosphere shielded canyons, about $15 \mathrm{ft}$ wide and over $550 \mathrm{ft}$ long. Cell exhaust utilizes sand filters. The facility is divided into "hot" and "warm" canyons. New hot side and warm side cranes were installed in 1989. The initial separation of uranium and neptunium from fission products was performed in the hot canyon; subsequent purification for each material was performed in the warm canyon. The primary separations equipment includes fuel and target dissolvers, centrifuges for solids removal, three cycles of solvent extraction equipment, process solution and waste evaporators, and various product and hold tanks.

Supporting Savannah River Site infrastructure includes the tank farms and Defense Waste Processing Facility, which may provide a means for the disposition of high-level waste generated from reprocessing.

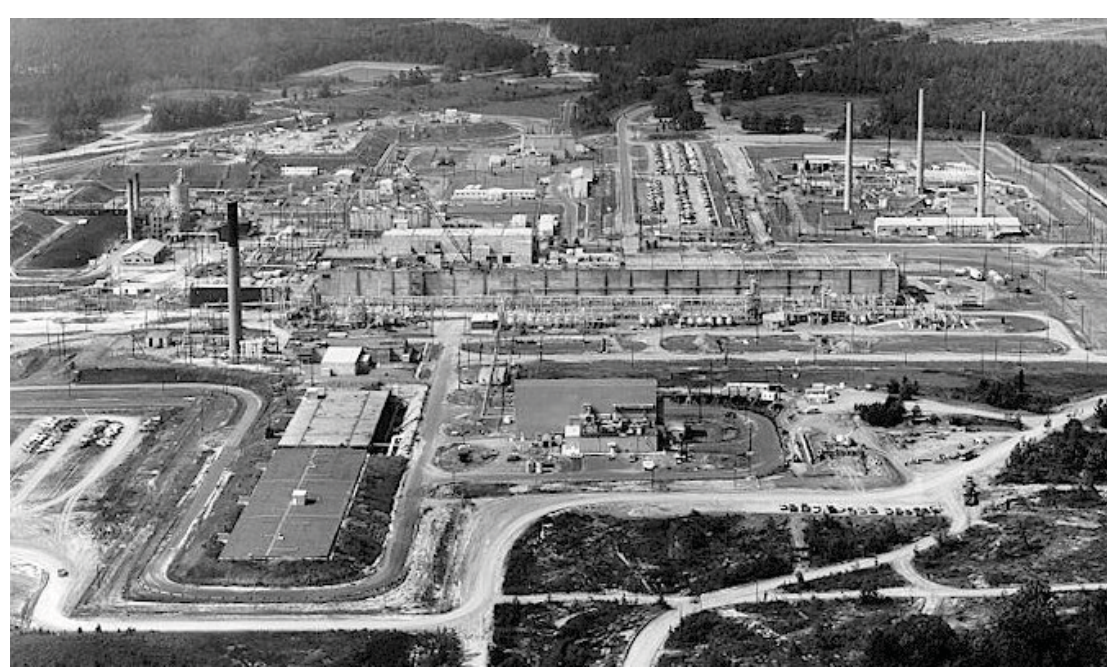

H-Canyon at the Savannah River Site However, while technically feasible, use of the Defense Waste Processing Facility may not be practical or allowed for non-defense R\&D waste. The $\mathrm{HB}$ line provides gloveboxes suitable for recovery of plutonium (including Pu-238) and conversion of the products into oxides for use in reactor fuels or thermoelectric devices. A chemistry laboratory is available to provide analytical support for technology research and demonstrations. Samples are transferred for analysis by truck. 


\section{Hot Fuel Examination Facility (HFEF)}

Location: Idaho National Laboratory

Currently Supporting: Multiple programs

Status: Fully operational

Essential Improvements: Complete safety analysis update

\begin{tabular}{|l|l|l|l|}
\hline Potential Program R\&D Applications are Shaded \\
\hline Light Water Reactors & $\begin{array}{l}\text { Irradiated Fuel } \\
\text { Separations }\end{array}$ & $\begin{array}{l}\text { Advanced Fuel } \\
\text { Development }\end{array}$ & Space Power Systems \\
\hline $\begin{array}{l}\text { High-Temperature } \\
\text { Reactor }\end{array}$ & Fast Spectrum Reactor & $\begin{array}{l}\text { Safeguards and } \\
\text { Security }\end{array}$ & $\begin{array}{l}\text { Modeling and } \\
\text { Simulation }\end{array}$ \\
\hline
\end{tabular}

HFEF is a large, heavily shielded hot cell facility designed to remotely characterize highly irradiated fuel and structural materials and have the flexibility to conduct numerous other research, development, and demonstration tasks as necessary. Currently, the facility is used for nondestructive examination, such as dimensional measurements, neutron radiography, and destructive examination, such as mechanical testing and metallographic/ceramographic characterization. Waste form development in support of advanced reprocessing research also is conducted in the cells. HFEF receives and disassembles full-size LWR assemblies and examines full-size LWR fuel elements. Thirty-foot long irradiated fuel sodium test

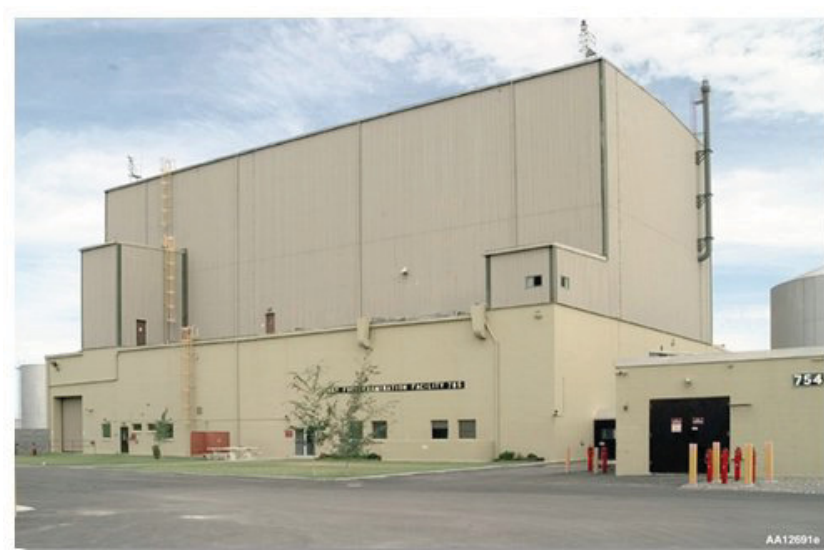

Hot Fuel Examination Facility at the Idaho National Laboratory loops have been disassembled and examined in HFEF. In addition, close to 1,000 drums of contact-handled transuranic waste have been characterized, core sampled, solidified, and repackaged in a waste characterization capability in the high-bay area of the facility.

HFEF consists primarily of two adjacent large, highly-shielded hot cells in a three-story building. The main cell (argon atmosphere) is $70 \mathrm{ft}$ long, $30 \mathrm{ft}$ wide, and $25 \mathrm{ft}$ high with 15 workstations, each with a viewing window and a pair of remote manipulators. A decontamination cell (air atmosphere) is $20 \mathrm{ft}$ long, $30 \mathrm{ft}$ wide, and $25 \mathrm{ft}$ high with six similarly equipped workstations. The cells are equipped with overhead cranes and overhead electromechanical manipulators. A Neutron

Radiography Reactor is located below the main cell for neutron radiography of irradiated fuels and materials test components. (See the Neutron Radiography Reactor Description Sheet.) There is a small shielded cell that houses an optical metallograph and a scanning electron microscope. HFEF is linked to the Materials and Fuels Complex analytical laboratories and the FCF by pneumatic sample transfer lines. 


\section{High Flux Isotope Reactor (HFIR)}

Location: Oak Ridge National Laboratory

Currently Supporting: Isotopes and neutron physics user experimentation

Status: Fully operational

\begin{tabular}{|l|l|l|l|}
\hline Potential Program R\&D Applications are Shaded \\
\hline Light Water Reactors & $\begin{array}{l}\text { Irradiated Fuel } \\
\text { Separations }\end{array}$ & $\begin{array}{l}\text { Advanced Fuel } \\
\text { Development }\end{array}$ & Space Power Systems \\
\hline $\begin{array}{l}\text { High-Temperature } \\
\text { Reactor }\end{array}$ & Fast Spectrum Reactor & $\begin{array}{l}\text { Safeguards and } \\
\text { Security }\end{array}$ & $\begin{array}{l}\text { Modeling and } \\
\text { Simulation }\end{array}$ \\
\hline
\end{tabular}

HFIR is a versatile, 85-MW, beryllium-reflected, light water-cooled and moderated, flux-trap type reactor that uses highly enriched uranium-235 as the fuel. The neutron scattering research facilities at HFIR contain a world-class collection of instruments used for fundamental and applied research on the structure and dynamics of matter. The reactor also is used for medical, industrial, and research isotope production; research on severe neutron damage to materials; and neutron activation to examine trace elements in the environment.

The original construction was finished in 1965; a project to enlarge in-reactor irradiation facilities to allow experiments to be instrumented was completed in 1986; and a cold neutron source was commissioned in 2007. HFIR is projected to continue operation through the year 2040 and beyond.

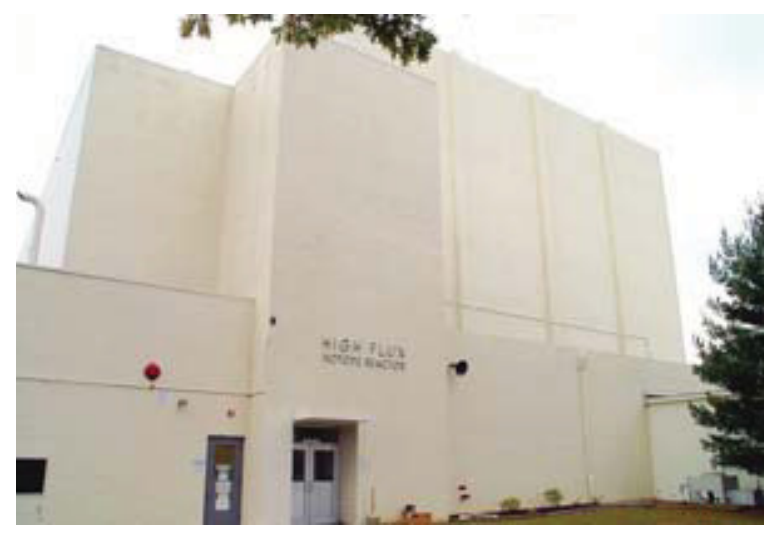

The High Flux Isotope Reactor at Oak Ridge National Laboratory
The reactor core consists of a series of concentric annular regions, each approximately $2 \mathrm{ft}(0.61 \mathrm{~m})$ high. A 5-in. (12.70-cm)-diameter hole, referred to as the "flux trap," forms the center of the core. The fuel region is surrounded by a concentric ring of beryllium reflector approximately $1 \mathrm{ft}(0.30 \mathrm{~m})$ thick. This, in turn, is subdivided into three regions: the removable reflector, the semi-permanent reflector, and the permanent reflector. The beryllium is surrounded by a water reflector of effectively infinite thickness. In the axial direction, the reactor is reflected by water.

In the flux trap in the center of the HFIR fuel element, a working thermal-neutron flux of 2E15 neutrons $/\left(\mathrm{cm}^{2} \cdot \mathrm{s}\right)$ is available for irradiation of experiments, including instrumented capsules and loops. The beryllium reflector also contains numerous experimental facilities with thermal-neutron fluxes up to $1 \mathrm{E} 15$ neutrons $/\left(\mathrm{cm}^{2} \cdot \mathrm{s}\right)$. These facilities can accommodate static experimental capsules, complex fuel-testing engineering loops, and special experimental isotope irradiations. Access to some experimental positions is via a pneumatic tube to allow rapid insertion and removal during reactor operation. 


\section{Irradiated Fuels Examination Laboratory}

Location: Oak Ridge National Laboratory

Currently Supporting: Multiple programs

Status: Operating

Remarks: Exhaust system and liquid waste improvements are needed

\begin{tabular}{|l|l|l|l|}
\hline Potential Program R\&D Applications are Shaded \\
\hline Light Water Reactors & $\begin{array}{l}\text { Irradiated Fuel } \\
\text { Separations }\end{array}$ & $\begin{array}{l}\text { Advanced Fuel } \\
\text { Development }\end{array}$ & Space Power Systems \\
\hline $\begin{array}{l}\text { High-Temperature } \\
\text { Reactor }\end{array}$ & Fast Spectrum Reactor & $\begin{array}{l}\text { Safeguards and } \\
\text { Security }\end{array}$ & $\begin{array}{l}\text { Modeling and } \\
\text { Simulation }\end{array}$ \\
\hline
\end{tabular}

The Irradiated Fuels examination Laboratory was constructed in 1963 and there have been no major changes; however, the equipment and building have been modernized through multiyear investments.

There are three main air atmosphere hot cells that are each $10 \mathrm{ft}$ deep and $14 \mathrm{ft}$ high. One is $24 \mathrm{ft}$ wide and the other two are $35.5 \mathrm{ft}$ wide. Each has $3 \mathrm{ft}$ of high-density concrete shielding and oil-filled lead glass

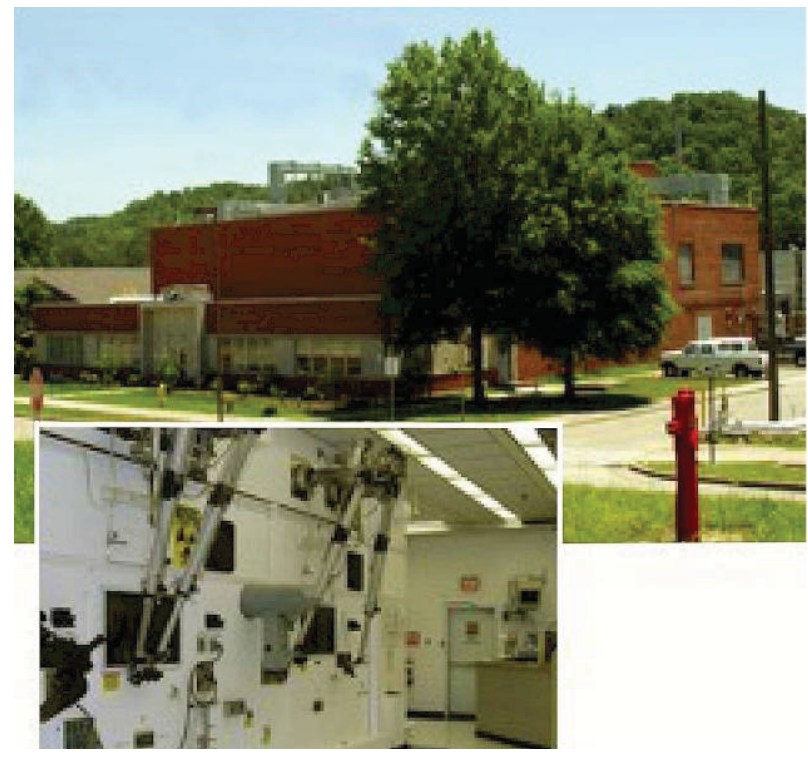

The Irradiated Fuels Examination Laboratory at Oak Ridge National Laboratory windows. The NAC and T-2 casks can be handled using air pallets to introduce irradiated items into the cells, including a single, full-length, commercial LWR fuel rod.

Also, there are three smaller stand-alone air atmosphere special purpose cells for irradiated microsphere gamma analysis; scanning electron microscopy; and core conduction cool-down testing. The irradiated microsphere gamma analysis and the scanning electron microscopy cells are 64 in. wide by 44 in. deep by 44 in. high with $8 \mathrm{in}$. of steel shielding and the core conduction cool-down testing cell is $4 \mathrm{ft}$ wide by $6 \mathrm{ft}$ deep by $8 \mathrm{ft}$ high with 4 in. of lead shielding. These cells have dry lead glass windows. 


\section{Irradiated Materials Examination and Testing Facility}

Location: Oak Ridge National Laboratory

Currently Supporting: Irradiated material property testing

Status: Operating

Remarks: Low-level liquid waste and hot cell exhaust system improvements are needed

\begin{tabular}{|l|l|l|l|}
\hline Potential Program R\&D Applications are Shaded \\
\hline Light Water Reactors & $\begin{array}{l}\text { Irradiated Fuel } \\
\text { Separations }\end{array}$ & $\begin{array}{l}\text { Advanced Fuel } \\
\text { Development }\end{array}$ & Space Power Systems \\
\hline $\begin{array}{l}\text { High-Temperature } \\
\text { Reactor }\end{array}$ & Fast Spectrum Reactor & $\begin{array}{l}\text { Safeguards and } \\
\text { Security }\end{array}$ & $\begin{array}{l}\text { Modeling and } \\
\text { Simulation }\end{array}$ \\
\hline
\end{tabular}

The Irradiated Materials Examination and Testing Facility is located at one end of Oak Ridge National Laboratory's Building 3025. It has six hot cells of various sizes from about $3 \mathrm{ft}$ wide by $4.5 \mathrm{ft}$ deep by $8 \mathrm{ft}$ high to $11 \mathrm{ft}$ wide by $7 \mathrm{ft}$ deep by $13 \mathrm{ft}$ high. Both concrete and steel are used for shielding. The floor and walls of one cell are covered with stainless steel. All cells have an air atmosphere and work is restricted to beta gamma radiation sources.

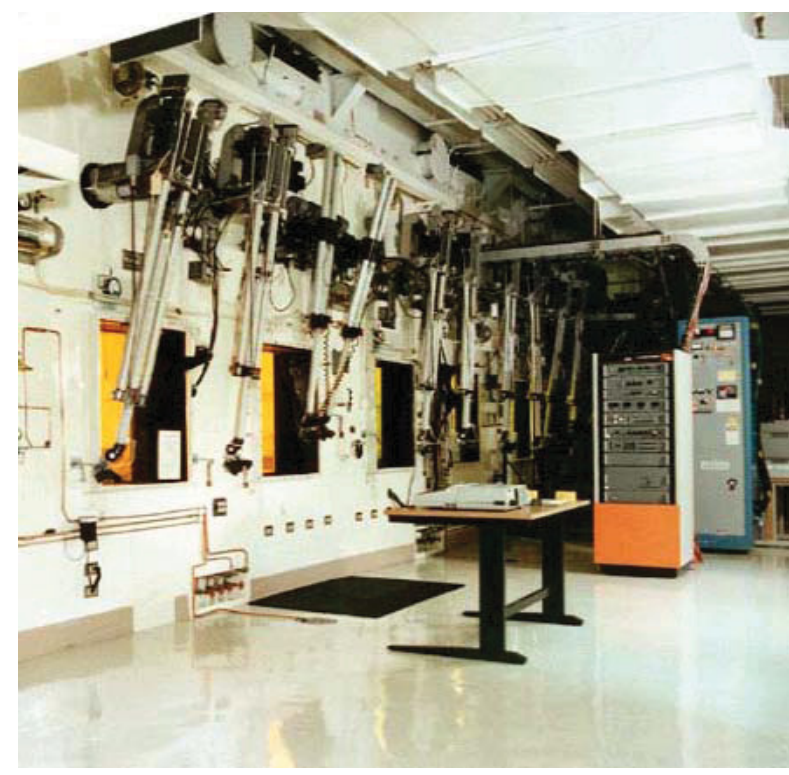

The Irradiated Materials Examination and Testing Facility at Oak Ridge National Laboratory
The current primary use of the Irradiated Materials Examination and Testing Facility is for conducting a variety of materials property tests (e.g., tensile, impact, hardness, compression, bending, fracture toughness, and crack growth) on irradiated samples under simulated temperature and pressure environments. Examination equipment includes profilometry and electron microscopy. However, the facility is flexible because the function of the cells is determined by the equipment installed.

Shielded storage is available in the floor of the cell access area. It consists of 15 stainless steel lined wells, which are each $10 \mathrm{ft}$ deep. They can be sealed from the general environment and are interconnected with a high-efficiency particulate air-filtered ventilation system. 


\section{ANL J, G, K, and M-Wing Facilities}

Location: Argonne National Laboratory

\section{Currently Supporting: Nuclear programs}

Status: J-wing: operating radiological facility; G-wing: ongoing downgrade to radiological facility; K-wing: operating, but currently closed to programmatic work; and M-wing: standby

Remarks: G-wing requires a reduction of radioisotope inventory to allow downgrading to radiological facility status

\section{Potential Program R\&D Applications are Shaded}

\begin{tabular}{|l|l|l|l|}
\hline Light Water Reactors & $\begin{array}{l}\text { Irradiated Fuel } \\
\text { Separations }\end{array}$ & $\begin{array}{l}\text { Advanced Fuel } \\
\text { Development }\end{array}$ & Space Power Systems \\
\hline $\begin{array}{l}\text { High-Temperature } \\
\text { Reactor }\end{array}$ & Fast Spectrum Reactor & $\begin{array}{l}\text { Safeguards and } \\
\text { Security }\end{array}$ & $\begin{array}{l}\text { Modeling and } \\
\text { Simulation }\end{array}$ \\
\hline
\end{tabular}

Argonne's J, G, K, and M-Wing Laboratories have the capability for handling and analyzing a wide range of radioactive materials in small quantities for laboratory-scale process development.

The laboratories in the $\mathrm{J}$ and $\mathrm{G}$-wings are used for process development in the areas of aqueous and electrochemical separations of nuclear materials. Several gloveboxes allow for safe handling of most radioactive materials. In addition, laboratories and instrumentation specifically for analysis of radiological materials are housed in the G-wing.

The radioactive materials inventory of G-wing is currently being reduced to allow its management as a radiological facility. This change in the status of the G-wing will not significantly change the capabilities of

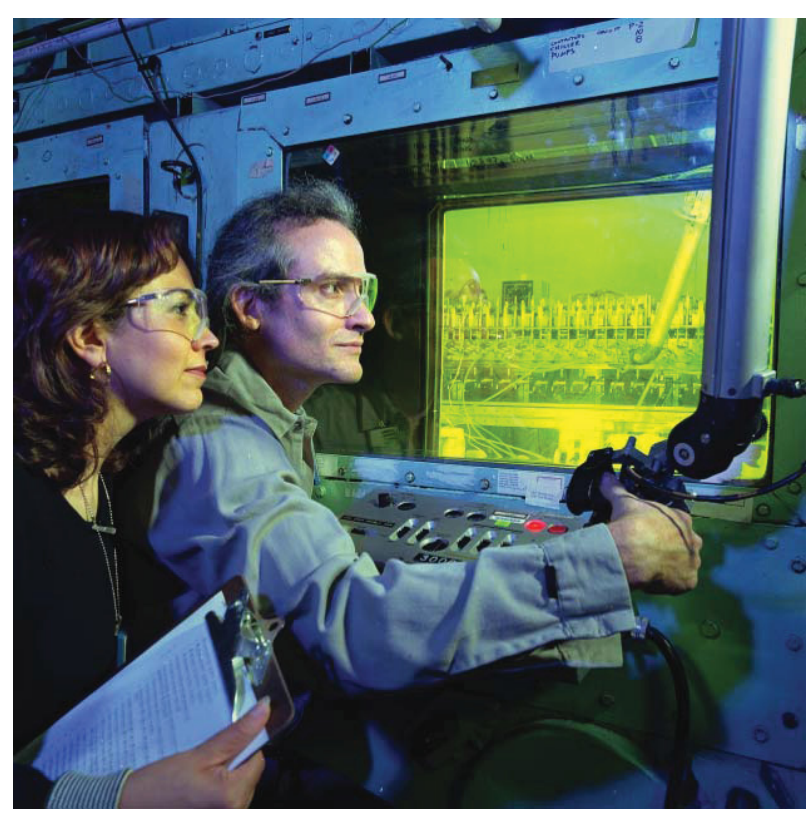

Safe material handling at Argonne National Laboratory the facility, but will limit the amount of radioactive materials present in the facility at any one time. Tracking and management of the inventory will allow a wide range of programmatic work to be accomplished.

The hot cells in the K-wing are currently operational, but closed to programmatic work. Several upgrades are being planned that would return the facility to full compliance and available for programmatic work. Two large cells with three telemanipulators are available; several different transfer mechanisms are also available.

The hot cells in the M-wing are currently in standby mode. A total of eight large cells (four on each of the two levels) are equipped with telemanipulators and adequate transfer equipment. Documentation and physical upgrades could bring this facility back into compliance. 


\section{Low-Activation Materials Development and Analysis Laboratory (LAMDA)}

Location: Oak Ridge National Laboratory

Currently Supporting: Multiple programs

Status: Operating

Remarks: Samples less than $60 \mathrm{mR} / \mathrm{hr}($ at $1 \mathrm{ft})$

\begin{tabular}{|l|l|l|l|}
\hline Potential Program R\&D Applications are Shaded \\
\hline Light Water Reactors & $\begin{array}{l}\text { Irradiated Fuel } \\
\text { Separations }\end{array}$ & $\begin{array}{l}\text { Advanced Fuel } \\
\text { Development }\end{array}$ & Space Power Systems \\
\hline $\begin{array}{l}\text { High-Temperature } \\
\text { Reactor }\end{array}$ & Fast Spectrum Reactor & $\begin{array}{l}\text { Safeguards and } \\
\text { Security }\end{array}$ & $\begin{array}{l}\text { Modeling and } \\
\text { Simulation }\end{array}$ \\
\hline
\end{tabular}

LAMDA is a multipurpose facility consisting of about $2,500 \mathrm{ft}^{2}$ in three radiological laboratories. LAMDA allows for examination of low-activation materials without the need for remote manipulation, which facilitates examination of more precise and delicate samples than possible in traditional hot cells.

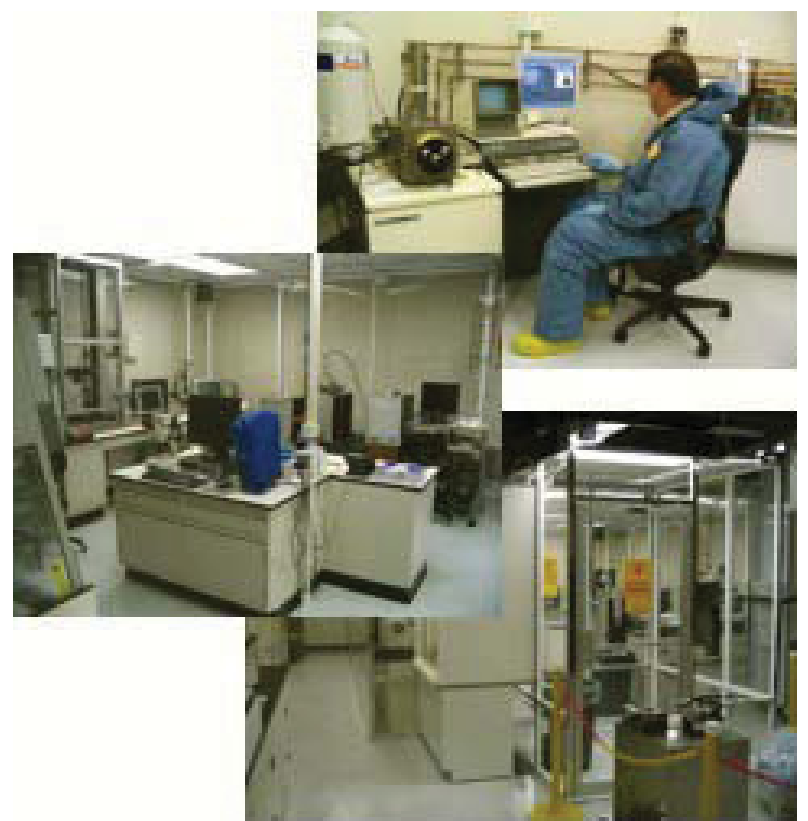

Low Activation Materials Development and Analysis Laboratory at the Oak Ridge National Laboratory
The three multipurpose radiological laboratories in LAMDA provide containment and shielding adequate for evaluation of materials with low radiological threat. As a guideline, the radiological hazards must be previously identified and the samples must have beta/gamma activity corresponding to a dose of less than $60 \mathrm{mR} / \mathrm{hr}$ (at $1 \mathrm{ft}$ ). Samples with low-level alpha contamination are acceptable, although alpha containing samples are considered non-routine and handled on a case-by-case basis.

Research encompasses basic science, applied engineering, and regulatory work, spanning materials for all past, present, and future reactor applications. The most commonly conducted work includes mechanical testing, optical and scanning electron microscopy, densitometry, metallography, and thermal and electrical conductivity. Low pressure (vacuum to 10-7 torr) and high temperature (to $1300^{\circ} \mathrm{C}$ ) testing is routine. Other activities not previously conducted are possible with the appropriate planning because equipment can be moved in and out of LAMDA on a campaign basis. 


\section{Materials and Fuels Complex Analytical Laboratory}

Location: Idaho National Laboratory

Currently Supporting: Multiple programs

Status: Fully operational

Remarks: Safety analysis update is in progress

\begin{tabular}{|l|l|l|l|}
\hline Potential Program R\&D Applications are Shaded \\
\hline Light Water Reactors & $\begin{array}{l}\text { Irradiated Fuel } \\
\text { Separations }\end{array}$ & $\begin{array}{l}\text { Advanced Fuel } \\
\text { Development }\end{array}$ & Space Power Systems \\
\hline $\begin{array}{l}\text { High-Temperature } \\
\text { Reactor }\end{array}$ & Fast Spectrum Reactor & $\begin{array}{l}\text { Safeguards and } \\
\text { Security }\end{array}$ & $\begin{array}{l}\text { Modeling and } \\
\text { Simulation }\end{array}$ \\
\hline
\end{tabular}

The Analytical Laboratory at the Idaho National Laboratory's Materials and Fuels Complex has been operating since 1960 with a major radiological filtration and exhaust system upgrade in 1994. It provides a wide range of analyses to support the missions of the Idaho National Laboratory's. The approximately $20,000 \mathrm{ft}^{2}$ of laboratory and office space houses six shielded hot cells; air and inert atmosphere gloveboxes; wet chemical laboratories; alpha, beta, and gamma counting facilities; a nuclear fuels casting laboratory; a nondestructive assay laboratory; and a wide variety of instrumentation. Direct connection of the Analytical Laboratory's hot cells to the FCF and the HFEF through a pneumatic transfer system allows for rapid, safe transfer of process or research and development samples to the laboratory for analysis.

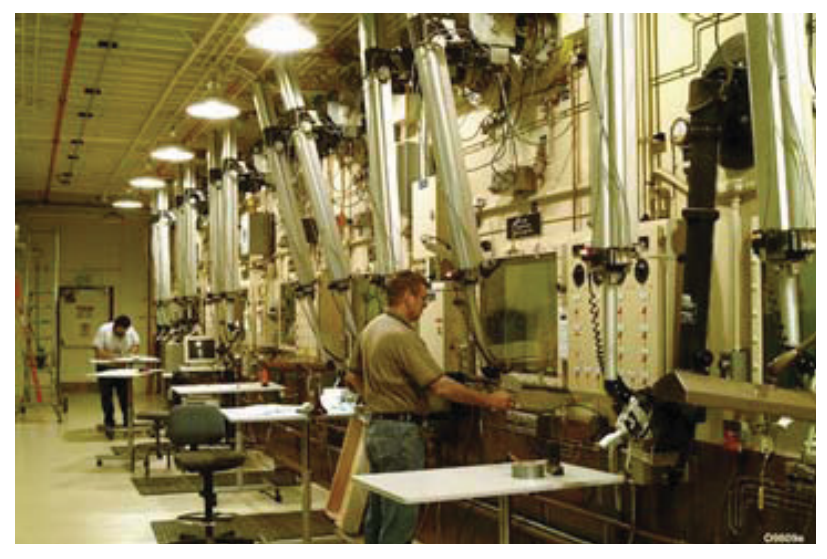

Materials and Fuels Complex Analytical Laboratory at the Idaho National Laboratory

and characterized for chemical, crystallographic, and thermal properties.
The shielded analytical hot cells are $5.5 \mathrm{ft}$ square and $12.5 \mathrm{ft}$ high for the receipt and analysis of highly radioactive samples. Samples can be directly transferred from the hot cells into a shielded glovebox equipped with an inductively coupled plasma atomic-emission spectrometer for analysis. Other types of samples can be transferred out of the hot cells into the B-wing for analysis by a variety of methods.

The laboratory excels at elemental and isotopic analysis of highly radioactive samples from major constituents to trace levels. The laboratories are equipped with a variety of sophisticated analytical instrumentation. In these cells and laboratories, materials, such as fuels and waste forms, are developed and thoroughly analyzed

Work has begun on a major addition to consolidate analytical capabilities from other areas of the Idaho National Laboratory and to house new state-of-the art equipment. This $8,200 \mathrm{ft}^{2}$ addition is expected to be operational in early 2009. 


\section{Material Sciences Laboratory}

Location: Pacific Northwest National Laboratory

Currently Supporting: Multiple programs

Status: Operating

Remarks: Capability will be relocated in 2011 as part of the Pacific Northwest National Laboratory Capability Replacement Project

Potential Program R\&D Applications are Shaded

\begin{tabular}{|l|l|l|l|}
\hline Light Water Reactors & $\begin{array}{l}\text { Irradiated Fuel } \\
\text { Separations }\end{array}$ & $\begin{array}{l}\text { Advanced Fuel } \\
\text { Development }\end{array}$ & Space Power Systems \\
\hline $\begin{array}{l}\text { High-Temperature } \\
\text { Reactor }\end{array}$ & Fast Spectrum Reactor & $\begin{array}{l}\text { Safeguards and } \\
\text { Security }\end{array}$ & $\begin{array}{l}\text { Modeling and } \\
\text { Simulation }\end{array}$ \\
\hline
\end{tabular}

The Material Sciences Laboratory is a recently renovated laboratory facility at the Hanford Site 300 Area north of Richland, Washington. It is operated for DOE by Pacific Northwest National Laboratory to measure the properties of radioactive and non-radioactive metals, ceramics, and liquids.

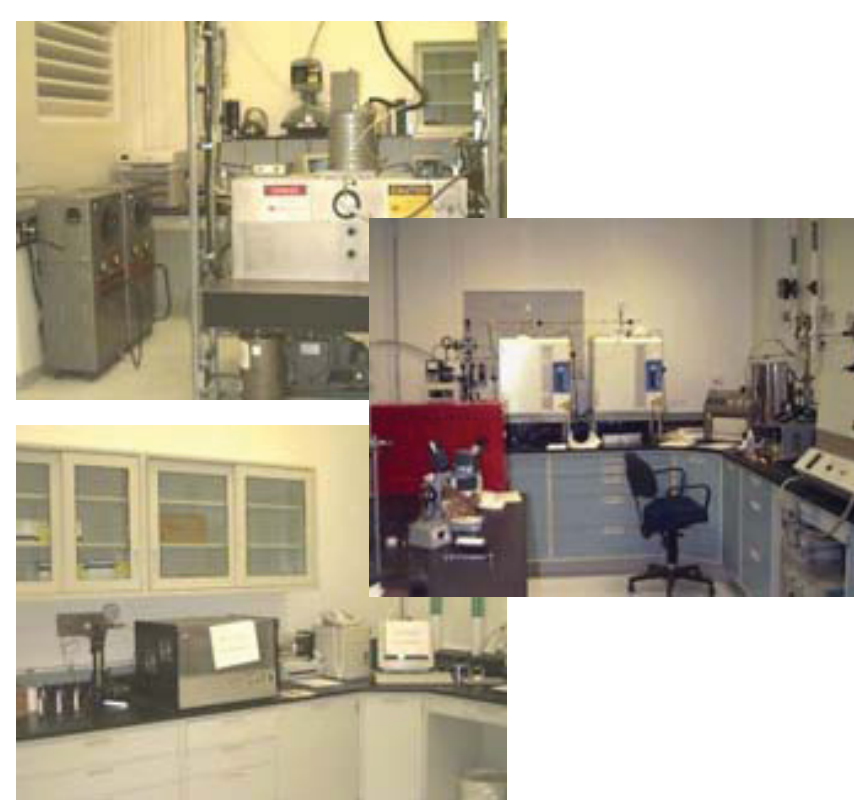

Examples of equipment in the Material Sciences Laboratory at Pacific Northwest National Laboratory
The Material Sciences Laboratory consists of four laboratories which include a wide array of gloveboxes, hoods, furnaces, and state-of-theart non-destructive measurement equipment (such as ultrasonic and eddy current devices). In these laboratories, researchers measure and create new ways to measure the properties of metals and liquids. Examples include measuring the density of radioactive waste slurry material; using ultrasonic techniques to evaluate grain structure, internal strain, and pressure effects of stress; and using advanced statistical methods to help analyze reactor pressure vessel fabrication processes to predict fabrication flaw rates.

In the Thermal Properties Laboratory, thermal diffusivity and thermal expansion test capabilities are available, in addition to a variety of metrology and sample preparation capabilities. The centerpiece of the Thermal Properties Laboratory is a laser flash thermal diffusivity measurement capability. Two diffusivity test units are available: (1) a

low-temperature apparatus for testing from room temperature to $400^{\circ} \mathrm{C}$ in air, and (2) a high-temperature unit (shown at above) for testing from 300 to $1500^{\circ} \mathrm{C}$ in vacuum or inert gas. Thermal diffusivity can be measured for engineering properties or radiation damage effects on either monolithic, isotropic composites, or layered materials. Samples can vary in size from 6 to $10 \mathrm{~mm}$ in diameter, and typical sample thicknesses range from 1 to $3 \mathrm{~mm}$. 


\section{Neutron Radiography Facility}

Location: Idaho National Laboratory

Currently Supporting: Multiple programs

Status: Fully operational

Remarks: Safety analysis update is in progress

\begin{tabular}{|l|l|l|l|}
\hline Potential Program R\&D Applications are Shaded \\
\hline Light Water Reactors & $\begin{array}{l}\text { Irradiated Fuel } \\
\text { Separations }\end{array}$ & $\begin{array}{l}\text { Advanced Fuel } \\
\text { Development }\end{array}$ & Space Power Systems \\
\hline $\begin{array}{l}\text { High-Temperature } \\
\text { Reactor }\end{array}$ & Fast Spectrum Reactor & $\begin{array}{l}\text { Safeguards and } \\
\text { Security }\end{array}$ & $\begin{array}{l}\text { Modeling and } \\
\text { Simulation }\end{array}$ \\
\hline
\end{tabular}

The Neutron Radiography Facility is a 250-kW TRIGA reactor located below the main cell of HFEF at the Idaho National Laboratory. The reactor, which began operation in 1977, is equipped with two beam tubes

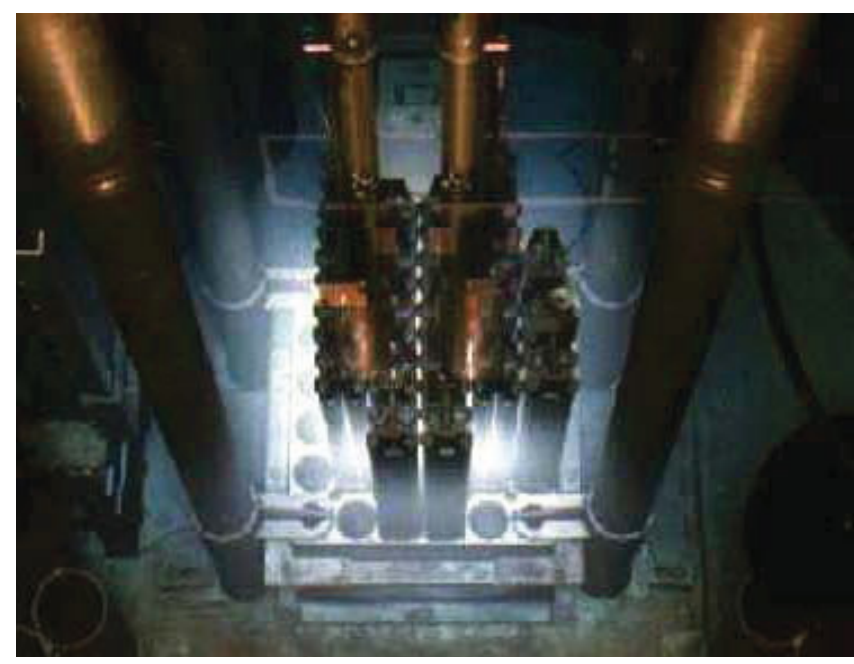

Core of the Neutron Radiography Facility at the Idaho National Laboratory and two separate radiography stations for radiography of irradiated or unirradiated components.

The primary radiography station is to perform neutron radiography of highly irradiated fuel elements, fuel subassemblies, and loop experiments without removing them from the HFEF hot-cell atmosphere. The secondary neutron radiography station is outside of the main cell and permits neutron radiography of either unirradiated or irradiated specimens without introducing them into the contaminated main cell.

In addition to its radiography capabilities, the Neutron Radiography Facility has in-core irradiation capabilities, including a water-filled port at the center of the core and a dry port at the edge of the core. A pneumatic transfer system will be installed soon for rapid transfer to and from the core. The Neutron Radiography Facility also operates a linear particle accelerator, which is currently used for nondestructive assay testing of nuclear waste and spent nuclear fuel. 


\section{New Waste Calcine Facility}

Location: Idaho National Laboratory

Currently Supporting: None

Status: Standby

Remarks: Restart with modifications and equipment for a new program will be needed

\begin{tabular}{|l|l|l|l|}
\hline Potential Program R\&D Applications are Shaded \\
\hline Light Water Reactors & $\begin{array}{l}\text { Irradiated Fuel } \\
\text { Separations }\end{array}$ & $\begin{array}{l}\text { Advanced Fuel } \\
\text { Development }\end{array}$ & Space Power Systems \\
\hline $\begin{array}{l}\text { High-Temperature } \\
\text { Reactor }\end{array}$ & Fast Spectrum Reactor & $\begin{array}{l}\text { Safeguards and } \\
\text { Security }\end{array}$ & $\begin{array}{l}\text { Modeling and } \\
\text { Simulation }\end{array}$ \\
\hline
\end{tabular}

The New Waste Calcine Facility was designed and constructed to solidify high-level liquid waste from processing spent nuclear fuels. Hot operations were conducted from 1982 until 1998 when the facility was placed in standby in accordance with a consent order between DOE and the State of Idaho.

The New Waste Calcine Facility is a five-level, $72,000 \mathrm{ft}^{2}$ facility with three main operating levels (one above grade and two below) for processing and decontamination functions. The decontamination area includes a shielded decontamination cell and the process area includes six shielded process cells for

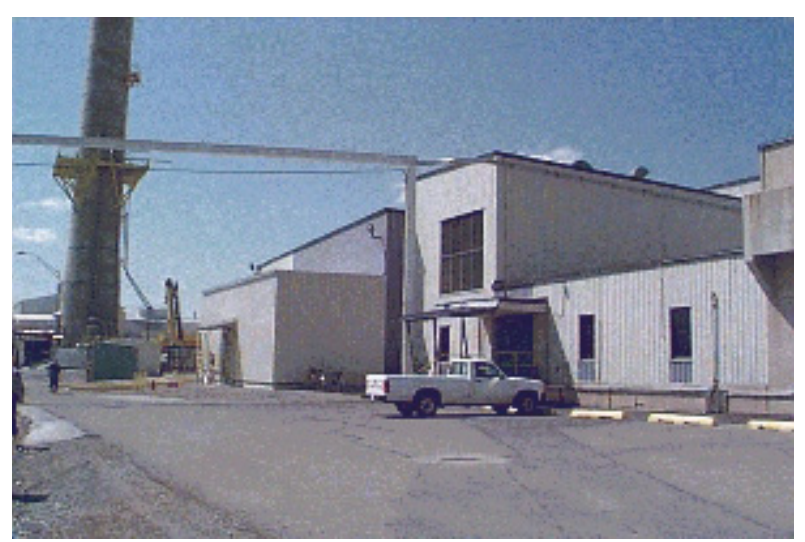

waste calcining. The facility is connected to the Remote Analytical Laboratory via a pneumatic transfer system. A dedicated emergency generator is housed in an adjacent hardened structure.

The calcining process was remotely controlled from a "hardened" control room in the above grade level and calcining took place in the two main below grade levels.

The decontamination cell interfaces with the remotely maintainable pump and valve corridor and the calciner cell for equipment maintenance.

New Waste Calcine Facility at the Idaho National Laboratory 


\section{Remote Analytical Laboratory (RAL)}

Location: Idaho National Laboratory

Currently Supporting: Idaho Completion Project, including waste certification for transuranic shipments to the Waste Isolation Pilot Plant

Status: In operation

Remarks: Safety analysis update is in progress

Potential Program R\&D Applications are Shaded

\begin{tabular}{|l|l|l|l|}
\hline Light Water Reactors & $\begin{array}{l}\text { Irradiated Fuel } \\
\text { Separations }\end{array}$ & $\begin{array}{l}\text { Advanced Fuel } \\
\text { Development }\end{array}$ & Space Power Systems \\
\hline $\begin{array}{l}\text { High-Temperature } \\
\text { Reactor }\end{array}$ & Fast Spectrum Reactor & $\begin{array}{l}\text { Safeguards and } \\
\text { Security }\end{array}$ & $\begin{array}{l}\text { Modeling and } \\
\text { Simulation }\end{array}$ \\
\hline
\end{tabular}

The Remote Analytical Laboratory is an analytical laboratory designed for a wide range of organic, inorganic, and radiochemical chemical analyses. This two-story, metal-clad, steel-framed, 13,000- $\mathrm{ft}^{2}$ facility contains a conventional chemical laboratory and an air atmosphere, analytical hot cell with a waste load-out cell.

The Analytical and Waste Handling Cells are constructed of reinforced concrete 36 in. thick and are on a separate foundation than the other facility structures. The analytical cell has six work stations with about $500 \mathrm{ft}^{2}$ of interior floor space for sample preparation and analysis. The waste load-out cell has two work stations with about $250 \mathrm{ft}^{2}$ of floor space. The analytical cell has pneumatic transfer lines to various nearby facilities, enabling radioactive samples to be pneumatically transferred and received. The Remote

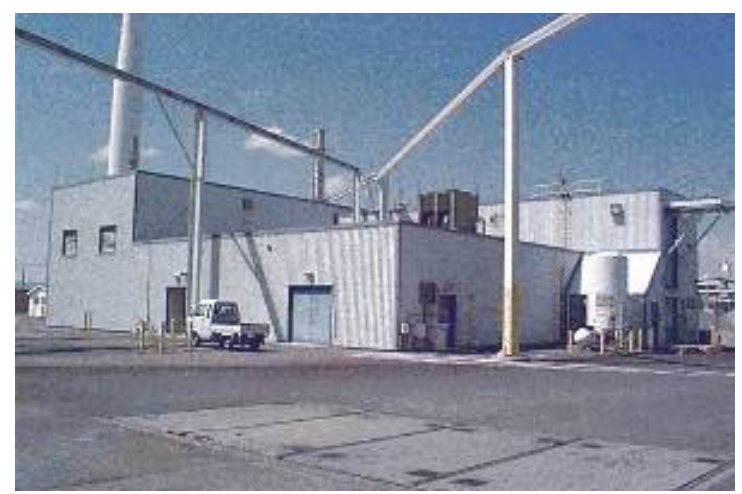

Remote Analytical Laboratory at the Idaho National Laboratory Site
Analytical Laboratory construction was completed in 1985 and upgraded in 1995.

The Remote Analytical Laboratory was designed to provide remote analytical support for spent nuclear fuel dissolution, reprocessing, and waste solidification. It currently provides analyses for radioactive and nonradioactive processes, waste characterization, and process development activities. The analytical cell provides the capability to perform chemical analysis on highly radioactive materials. There also are work areas for bench-scale development work.

Analytical processes for metals and organics have been qualified to provide certification of waste for Waste Isolation Pilot Plant disposal in support of the National Transuranic Program. 


\section{Receiving Basin for Offsite Fuel}

Location: Savannah River Site

Currently Supporting: None

Status: Shutdown/standby

Remarks: Refurbishment and restart needed

\begin{tabular}{|l|l|l|l|}
\hline Potential Program R\&D Applications are Shaded \\
\hline Light Water Reactors & $\begin{array}{l}\text { Irradiated Fuel } \\
\text { Separations }\end{array}$ & $\begin{array}{l}\text { Advanced Fuel } \\
\text { Development }\end{array}$ & Space Power Systems \\
\hline $\begin{array}{l}\text { High-Temperature } \\
\text { Reactor }\end{array}$ & Fast Spectrum Reactor & $\begin{array}{l}\text { Safeguards and } \\
\text { Security }\end{array}$ & $\begin{array}{l}\text { Modeling and } \\
\text { Simulation }\end{array}$ \\
\hline
\end{tabular}

The Receiving Basin for Offsite Fuel is a facility of about $15,000 \mathrm{ft}^{2}$ and includes an unloading basin, two storage basins, a repackaging basin, a disassembly basin, and an under-water fuel cutting and inspection basin. It was operated as a Category II facility and provided for the safe receipt and interim storage of irradiated spent nuclear fuel from Savannah River Site reactors and other domestic and foreign test and research reactors. It began operation in 1964. All utilities were disconnected in 2004 and the facility was shutdown with no routine personnel access.

The basins and their interconnecting transfer canals held about 500,000 gallons of water and have the capacity for storage of about 400 commercial fuel assemblies. Access for shipments is by either by rail or

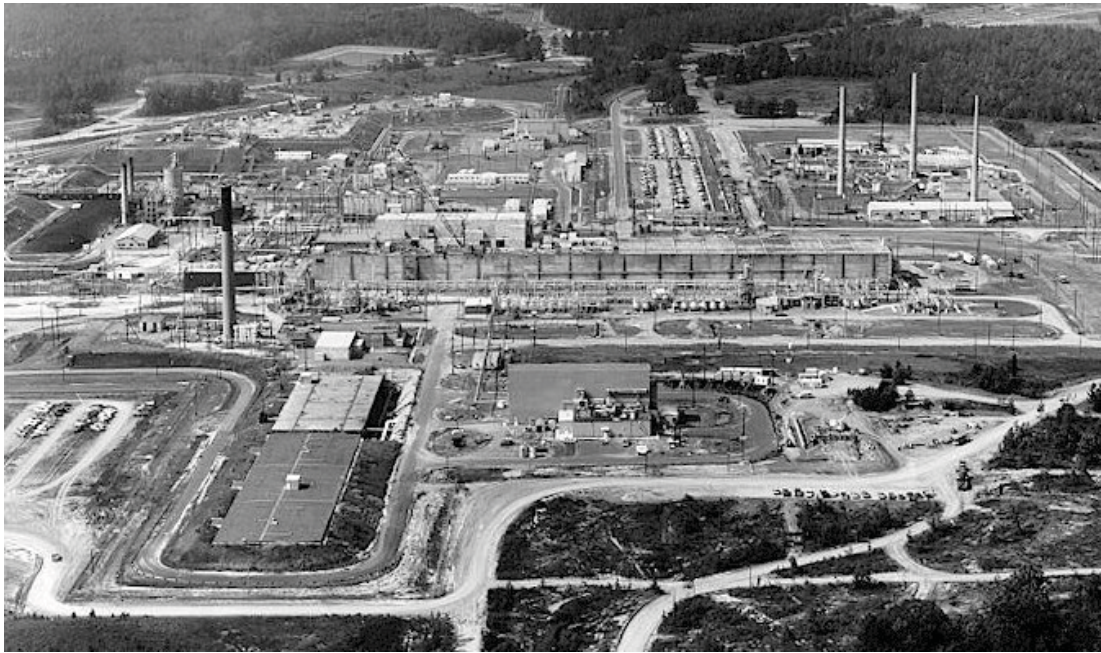

The Receiving Basin for Offsite Fuel is located in the 200-H Chemical Separation Area at the Savannah River Site truck. The facility has a 100-ton overhead trolley crane.

Basins are epoxy lined with stainless steel floors and up to $29 \mathrm{ft}$ deep with $45-\mathrm{ft}$ deep wells. Storage racks are slotted due to limited basin depth, and receipt of commercial fuel requires a "coffin-type" cask and a strong-back device for handling fuel bundles horizontally.

Refurbishment, modification, and restart for commercial fuel receipt and storage was recently estimated to require $\$ 25$ to $\$ 30$ million. 


\section{Radiochemical Engineering Development Center}

Location: Oak Ridge National Laboratory

Currently Supporting: High Flux Isotope Reactor target preparation and processing and aqueous process development

Status: Operating

Remarks: Volatile off-gas system replacement is needed

Potential Program R\&D Applications are Shaded

\begin{tabular}{|l|l|l|l|}
\hline Light Water Reactors & $\begin{array}{l}\text { Irradiated Fuel } \\
\text { Separations }\end{array}$ & $\begin{array}{l}\text { Advanced Fuel } \\
\text { Development }\end{array}$ & Space Power Systems \\
\hline $\begin{array}{l}\text { High-Temperature } \\
\text { Reactor }\end{array}$ & Fast Spectrum Reactor & $\begin{array}{l}\text { Safeguards and } \\
\text { Security }\end{array}$ & $\begin{array}{l}\text { Modeling and } \\
\text { Simulation }\end{array}$ \\
\hline
\end{tabular}

The Radiochemical Engineering Development Center, constructed in the mid 1960s, includes two Hazard Category II, Security Category IV hot-cell facilities (Buildings 7920 and 7930), plus support facilities.

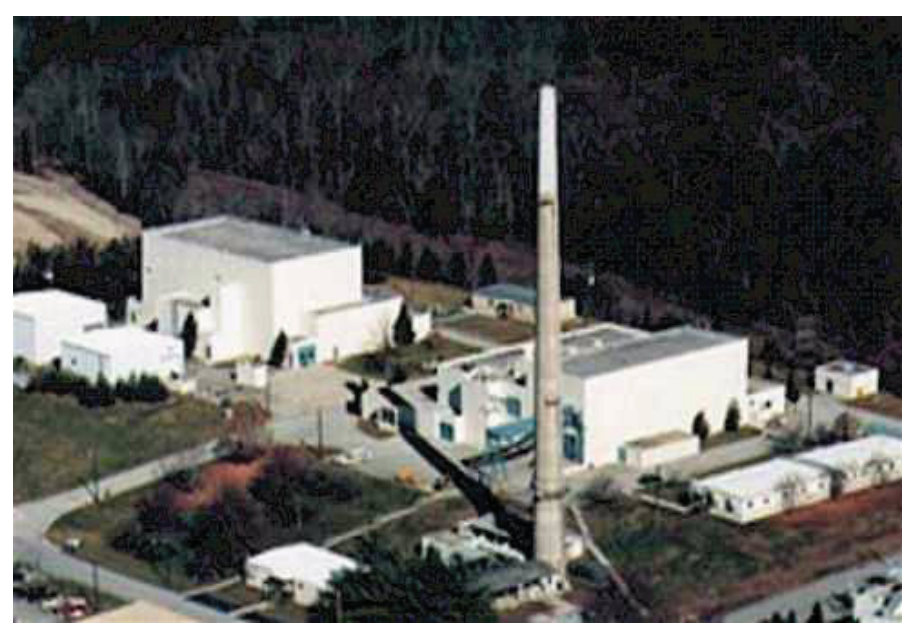

The Radiochemical Engineering Development Center at Oak Ridge National Laboratory
Building 7920 includes a series of nine 7 -ft by 7-ft by 8.5 -ft high air atmosphere hot cells with shield window and master/slave manipulators. Also, there are tank and waste pits of various sizes, a transfer cubicle, two caves 3.5-ft high, and a 4 -ft wide by 3 -ft deep by 4 -ft high target decontamination cell. In addition, the facility includes analytical chemistry laboratories.

The hot cells were originally built in the 1960s to fabricate and process High Flux Isotope Reactor plutonium, americium, and curium targets, and to separate californium and other rare heavy actinides. It is still used for this mission, but the throughput requires processing only on the order of once per year. One cell is currently being used to demonstrate the UREX aqueous separations

process using fuel that is chopped in the IFEL as part of a coupled end-to-end demonstration. Competing programs leave little space available in Building 7920.

Building 7930 includes seven relatively large, air atmosphere hot cells. Cell A is 8 -ft deep by 20 -ft long by 24- $\mathrm{ft} \mathrm{high} \mathrm{and} \mathrm{unshielded.} \mathrm{Cell} \mathrm{B} \mathrm{is} 16 \mathrm{ft}$ by $23 \mathrm{ft}$ by $22.5 \mathrm{ft}$ with concrete shielding and a stainless steel liner. Cell $C$ is $20 \mathrm{ft}$ by $33 \mathrm{ft}$ by $24 \mathrm{ft}$ with concrete shielding and a stainless steel liner. Cell $D$ is $20 \mathrm{ft}$ by $41 \mathrm{ft}$ by $13 \mathrm{ft}$ with concrete shielding and a stainless steel liner. Cell $E$ is $20 \mathrm{ft}$ by $17 \mathrm{ft}$ by $30 \mathrm{ft}$ with concrete shielding. A stainless steel liner is on the floor and $1 \mathrm{ft}$ up the walls. Cell $F$ is $15 \mathrm{ft}$ by $37 \mathrm{ft}$ by $13 \mathrm{ft}$ with concrete walls. It is operated as a spent nuclear material storage vault. Cell $\mathrm{G}$ is $20 \mathrm{ft}$ by $17 \mathrm{ft}$ by $30 \mathrm{ft}$ with concrete shielding and a stainless steel liner. Cells $D$ and $E$ have never been used and Cells $A$ and $B$ are operated as contamination free cells. Also, there is a small fuel storage pool that is $22 \mathrm{ft}$ deep. 


\section{Radiochemical Processing Laboratory}

Location: Pacific Northwest National Laboratory

Currently Supporting: Multiple programs

Status: Fully operational

Remarks: Life-extension upgrades are a part of the Pacific Northwest National Laboratory Capability Replacement Project

Potential Program R\&D Applications are Shaded

\begin{tabular}{|l|l|l|l|}
\hline Light Water Reactors & $\begin{array}{l}\text { Irradiated Fuel } \\
\text { Separations }\end{array}$ & $\begin{array}{l}\text { Advanced Fuel } \\
\text { Development }\end{array}$ & Space Power Systems \\
\hline $\begin{array}{l}\text { High-Temperature } \\
\text { Reactor }\end{array}$ & Fast Spectrum Reactor & $\begin{array}{l}\text { Safeguards and } \\
\text { Security }\end{array}$ & $\begin{array}{l}\text { Modeling and } \\
\text { Simulation }\end{array}$ \\
\hline
\end{tabular}

The Radiochemical Processing Laboratory at the Pacific Northwest National Laboratory is Hazard Category 2 non-reactor facility. The facility is approximately $145,000 \mathrm{ft}^{2}$ and contains 12 small hot cells (about $850 \mathrm{ft}^{2}$ of floor space total) in two hot-cell annexes plus three smaller modular hot cells, multiple gloveboxes, and general purpose radiochemical research laboratories. Two additional small hot cells, four additional modular hot cells, and four gloveboxes will be added to the facility as part of 20-year life-extension project with completion expected in the year 2011.

The High-Level Radiochemistry Facility hot cell annex includes three hot cells designed to shield $10^{6} \mathrm{R} / \mathrm{hr}$. The High-Level Radiochemistry Facility previously housed a spent fuel reprocessing line in support of the Nuclear Waste Vitrification Program. An adjacent truck lock and 30-ton capacity bridge crane with a 5-ton capacity auxiliary hook support fuel cask receipt in the High-Level Radiochemistry Facility. Full-length

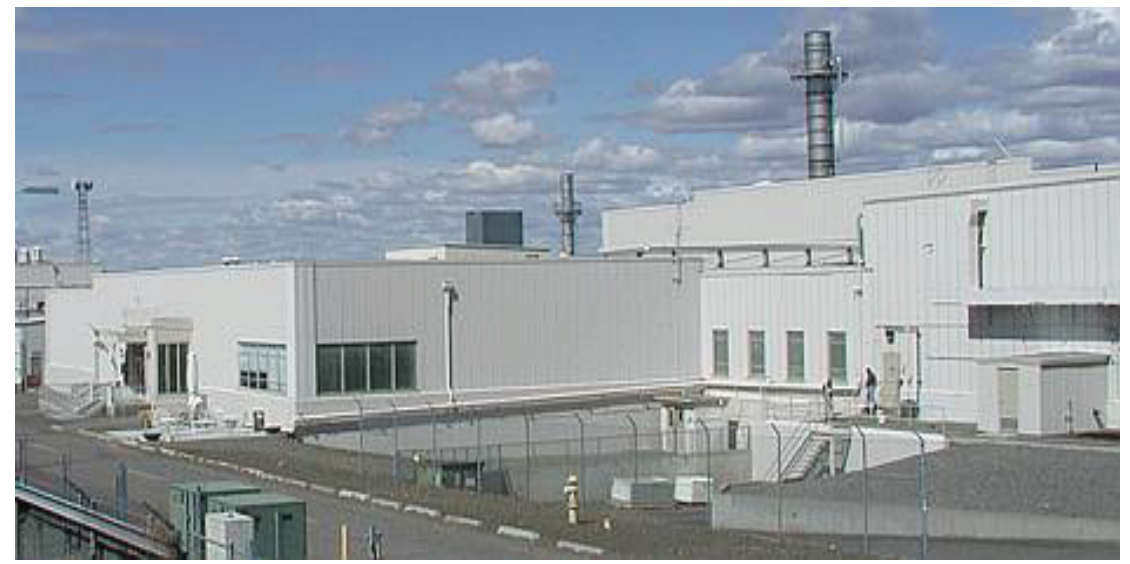

Radiochemical Processing Laboratory at the Pacific Northwest National Laboratory commercial reactor rods have been received and destructively examined in these cells. The Shielded Analytical Laboratory hot cell annex contains six interconnecting hot cells that handle radioactive samples up to $2000 \mathrm{R} / \mathrm{hr}$.

Wet laboratories in the center of the facility support bench-scale radiochemical processing and separations research. A shielded glovebox contains a bank of sixteen $2-\mathrm{cm}$ centrifugal contactors that enable solvent extraction research. A fuels development and evaluation laboratory contains an oxide fuel fabrication line to produce research-grade fuels. The Radiochemical Processing Laboratory also includes irradiated materials destructive examination capabilities; thermal analysis equipment to evaluate thermo-physical properties of advanced fuels and irradiated materials, and a state-of-the-art flow-through system to evaluate the stability and durability of waste forms. 


\section{Shielded Cells Facility-High-Level Cells}

Location: Savannah River National Laboratory

Currently Supporting: Site cleanup waste characterization and process development

Status: Operating

Remarks: Degraded windows were recently replaced.

\begin{tabular}{|l|l|l|l|}
\hline Potential Program R\&D Applications are Shaded \\
\hline Light Water Reactors & $\begin{array}{l}\text { Irradiated Fuel } \\
\text { Separations }\end{array}$ & $\begin{array}{l}\text { Advanced Fuel } \\
\text { Development }\end{array}$ & Space Power Systems \\
\hline $\begin{array}{l}\text { High-Temperature } \\
\text { Reactor }\end{array}$ & Fast Spectrum Reactor & $\begin{array}{l}\text { Safeguards and } \\
\text { Security }\end{array}$ & $\begin{array}{l}\text { Modeling and } \\
\text { Simulation }\end{array}$ \\
\hline
\end{tabular}

The Shielded Cells Facility high-level hot cells consist of two cell blocks containing a total of 16 small, stainless steel lined, air atmosphere hot cells with a two-operator position in each. Each cell module consists of an operating space approximately 6 -ft wide $\times 6$ - $\mathrm{ft}$ deep $\times 15$-ft high with shielding walls. The shielding is designed to protect operating personnel from $1 \mathrm{MeV}$ gamma radiation from a $10,000 \mathrm{Ci}$ source. Plutonium inventory is limited to $300-400 \mathrm{~g}$ per cell block. The operator views the hot cell operations through a leaded glass window to perform tasks with manipulators.

The cell modules within each cell block are interconnected. Some walls can be removed to combine up to three cells. Overhead, in-cell, 1-ton bridge mounted cranes are used to move materials between the interconnected cells. The top and rear walls of the shielded cells are in a high-bay loading area with a

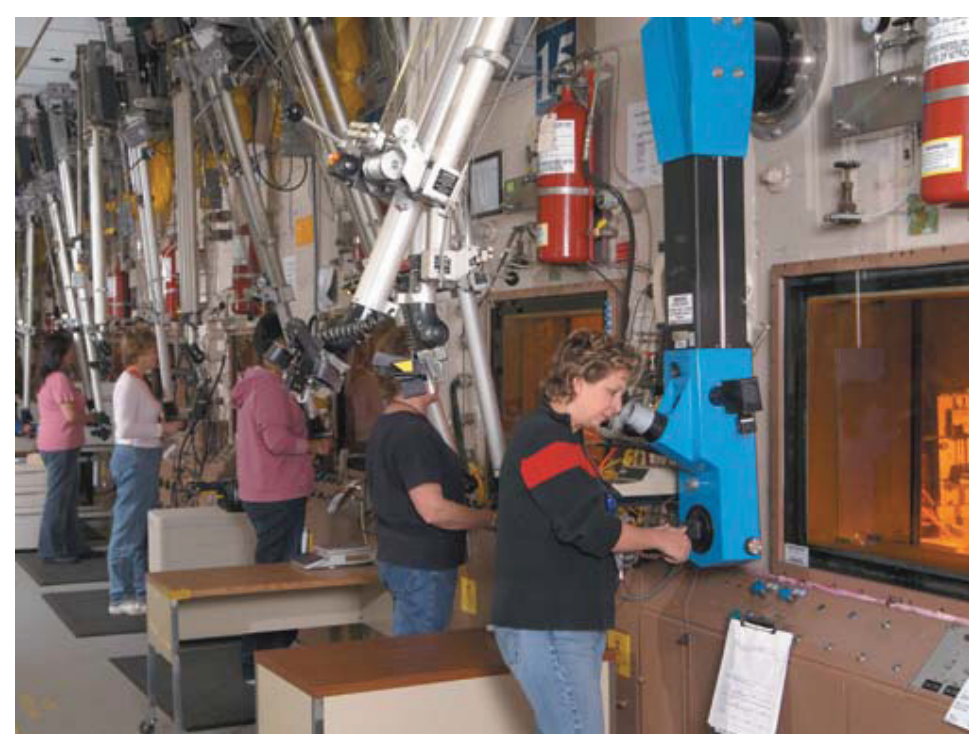

In addition to the manipulators, the shielded cells include equipment for a variety of analytical and research tasks 10-ton crane to facilitate the transfer of materials and equipment into and out of the cells. The cells are designed to allow easy modifications and the change out of processing equipment. Standard equipment and sample input to the cells fits within a 10-in. x 11-in. transfer port envelope. Larger items require removal of the cell roof hatch.

The Shielded Cells Facility includes support and auxiliary facilities that assist in cell operations. These facilities include a mockup shop used to functionally test equipment before it is placed into radioactive service and maintenance facilities for the decontamination and repair of manipulators. 


\section{Sol Gel Laboratory Facility}

Location: Building 4501, Oak Ridge National Laboratory

Currently Supporting: Fuel cycle and non-proliferation

Status: Fully operational

\begin{tabular}{|l|l|l|l|}
\hline Potential Program R\&D Applications are Shaded & Space Power Systems \\
\hline Light Water Reactors & $\begin{array}{l}\text { Irradiated Fuel } \\
\text { Separations }\end{array}$ & $\begin{array}{l}\text { Advanced Fuel } \\
\text { Development }\end{array}$ & $\begin{array}{l}\text { Modeling and } \\
\text { Simulation }\end{array}$ \\
\hline $\begin{array}{l}\text { High-Temperature } \\
\text { Reactor }\end{array}$ & Fast Spectrum Reactor & $\begin{array}{l}\text { Safeguards and } \\
\text { Security }\end{array}$ \\
\hline
\end{tabular}

The Oak Ridge National Laboratory's Sol Gel Laboratories provide R\&D scale facilities for handing and processing numerous radioactive and non-radioactive materials. These laboratories include facilities for preparation of precursor materials and feed stocks, formation and curing of microspheres and fuel kernels, sorting and classification of microspheres, microscopic inspection, physical property determination, waste consolidation, and reagent recycle. Numerous types of microspheres with precisely controlled properties can be produced via the sol gel process. Microspheres ranging in size from 40 to

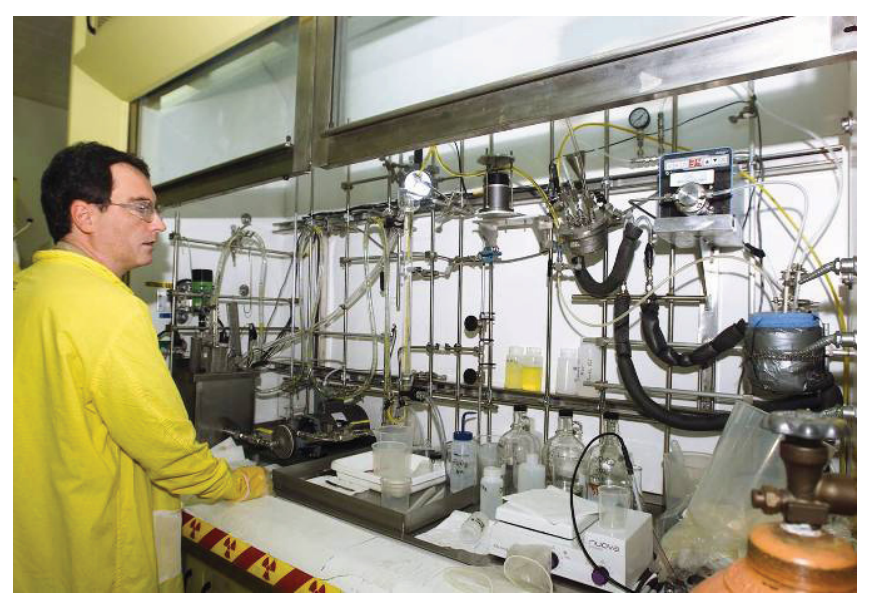

Sol Gel Laboratory at Oak Ridge National Laboratory $1200 \mu \mathrm{m}$ can be produced via traditional sol gel technology. The smaller diameter microspheres were produced using a piezoelectric feed system.

Since the late 1970s, Oak Ridge National Laboratory researchers have studied and fully developed the internal gelation process for making $\mathrm{UO}_{2},(\mathrm{U}, \mathrm{Pu}) \mathrm{O}_{2}$, and $\left(\mathrm{UO}_{2}+\mathrm{UC}_{2}\right)$ microspherical fuels. In the 1990 s, Oak Ridge National Laboratory extended the boundaries of the technology by developing the process to make hydrous metal oxide spheres of $\mathrm{Ti}, \mathrm{Zr}, \mathrm{Fe}$, and other cations for such uses as sorbents, waste forms, catalysts, getters, and dielectrics. More recently, $2 \mathrm{~kg}$ of $\mathrm{UO}_{2}$ kernels with diameters of $500 \pm 20 \mu \mathrm{m}$ and $3.5 \mathrm{~kg}$ of $\mathrm{UO}_{2}$ kernels with diameters of $350 \pm 10 \mu \mathrm{m}$ were

prepared for the Advanced Gas-Cooled Reactor Program. These microspheres were used in the triisotropic (TRISO) coating development. In addition, $\mathrm{UO}_{2}+\mathrm{UC}_{2}$ fuel kernels were produced and evaluated for the International Nuclear Energy Research Initiative.

The internal gelation process used is one of the sol-gel processes developed for preparation of microspheres of nuclear fuel in which chilled clear broth droplets containing acid-deficient uranyl nitrate, hexamethylenetetramine, and urea are heated, causing homogenous gelation and solidification of the droplets that, after washing treatments, can be dried calcined and sintered to ceramic kernels of the required density. 


\section{Space and Security Power Systems Facility}

Location: Idaho National Laboratory

Currently Supporting: Space and security missions

Status: Fully operational

Potential Program R\&D Applications are Shaded

\begin{tabular}{|l|l|l|l|}
\hline Light Water Reactors & $\begin{array}{l}\text { Irradiated Fuel } \\
\text { Separations }\end{array}$ & $\begin{array}{l}\text { Advanced Fuel } \\
\text { Development }\end{array}$ & Space Power Systems \\
\hline $\begin{array}{l}\text { High-Temperature } \\
\text { Reactor }\end{array}$ & Fast Spectrum Reactor & $\begin{array}{l}\text { Safeguards and } \\
\text { Security }\end{array}$ & $\begin{array}{l}\text { Modeling and } \\
\text { Simulation }\end{array}$ \\
\hline
\end{tabular}

The Space and Security Power Systems Facility consists of an administrative building and a nuclear process building. The facility is located at Materials and Fuels Complex at the Idaho National Laboratory. The nuclear process building is a two-story, reinforced concrete building of approximately 10,000 gross $\mathrm{ft}^{2}$. It is a security access-controlled facility surrounded by the Materials and Fuels Complex Perimeter Intrusion Detection and Assessment System. Modification of the administrative building and construction

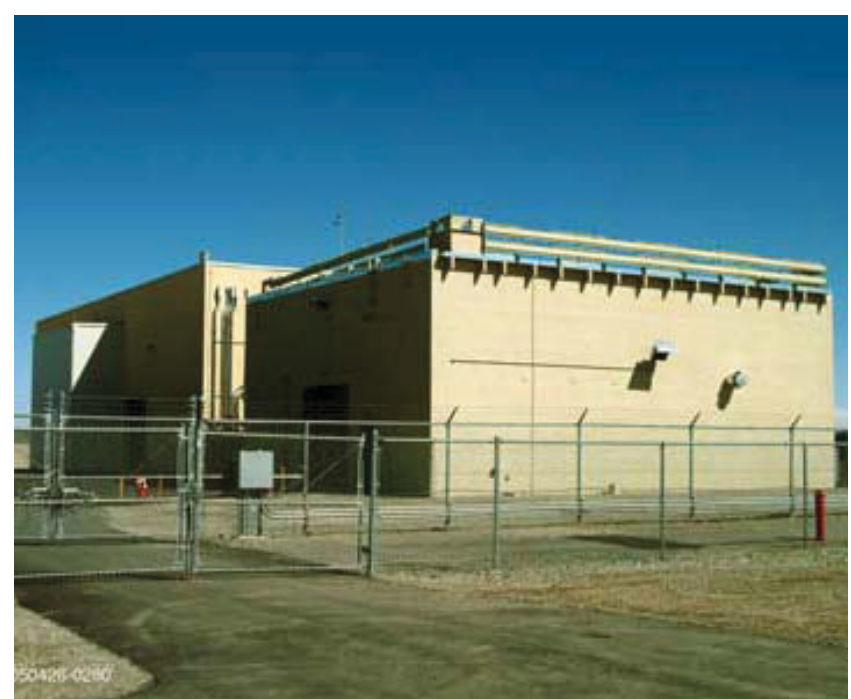

Space and Security Power Systems Facility at the Idaho National Laboratory of the nuclear process building was completed in 2004.

The Space and Security Power Systems Facility provides the capability for assembly and testing of radioisotope power sources. These power sources, or batteries, convert the heat from Pu-238 decay to electrical power for use in space applications or remote terrestrial locations. After a power source is assembled in one of the specialized gloveboxes in the facility, testing is performed to ensure the power source will work in the desired environment. Testing activities include the following: vibration testing to simulate lift-off conditions for space applications or vehicle transport conditions for terrestrial missions; mass properties determination for space and terrestrial applications; and thermal/vacuum testing that duplicates, as close as possible on earth, the conditions under which a space battery will be expected to operate. 


\section{Safety and Tritium Applied Research Facility}

Location: Idaho National Laboratory

Currently Supporting: Fusion reactor safety

Status: Operating

Remarks: Upgrade completed in 2002

\begin{tabular}{|l|l|l|l|}
\hline Potential Program R\&D Applications are Shaded \\
\hline Light Water Reactors & $\begin{array}{l}\text { Irradiated Fuel } \\
\text { Separations }\end{array}$ & $\begin{array}{l}\text { Advanced Fuel } \\
\text { Development }\end{array}$ & Space Power Systems \\
\hline $\begin{array}{l}\text { High-Temperature } \\
\text { Reactor }\end{array}$ & Fast Spectrum Reactor & $\begin{array}{l}\text { Safeguards and } \\
\text { Security }\end{array}$ & $\begin{array}{l}\text { Modeling and } \\
\text { Simulation }\end{array}$ \\
\hline
\end{tabular}

The Safety and Tritium Applied Research Facility is a low-hazard, radiological facility comprised of two interconnected buildings (TRA-666 and TRA-666A), with a total of about 4,400 $\mathrm{ft}^{2}$ distributed between four laboratory rooms, a high bay, and a small office room. It was constructed in 1963 with major modifications in 1986 and 2002. It is equipped with laboratory infrastructure and experimental systems to support tritium science and fusion technology research activities related to development of safe and environmentally friendly fusion energy. The tritium inventory in the Safety and Tritium Applied Research Facility is controlled to be less than $15,000 \mathrm{Ci}$. The Safety and Tritium Applied Research Facility is a designated a DOE National User Facility.

Experimental systems and tritium process equipment are located in the laboratories and a high-bay area. These systems support both tritium and non-tritium research (bench-scale experiments and engineering tests). Research capabilities include (1) hydrogen/tritium plasma-surface interaction with materials, (2) chemical reactivity and oxidation of plasma-facing materials, (3) mobilization of activation products

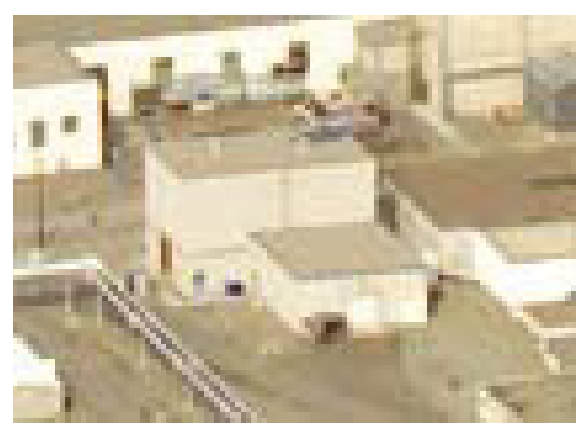

Safety and Tritium Applied Research Facility at the Idaho National Laboratory and tritium, (4) dust generation and characterization for fusion devices, and (5) molten salts for tritium breeder and coolant applications. Two experimental systems that support plasma-surface interaction research are the $\mathrm{D}$-ion implantation system, and the tritium plasma experiment. Research on Li-Be fluoride salts is conducted with inert gas, glovebox-based experimental systems.

TRA-666 and TRA-666A are equipped with separate heating, ventilation, and air conditioning systems that provide once-through air in each building with the air eventually exhausted to the site exhaust stack. This feature enables tritium operations to be localized primarily in TRA-666 and non-tritium operations in TRA-666A. To support tritium operations in TRA-666, the facility is equipped with gloveboxes and a tritium cleanup system to process gas streams from gloveboxes and experimental systems using tritium and tritium monitoring instrumentation (e.g., room air, glovebox atmosphere, and laboratory exhaust). To support research activities with hazardous and environmentally sensitive materials, TRA-666A is equipped with several gloveboxes filled with inert gas. For example, research with beryllium and materials containing beryllium has been ongoing for years in a safe and productive manner. 


\section{TA-55 Plutonium Facility}

Location: Los Alamos National Laboratory

Currently Supporting: Multiple programs

Status: Fully operational

Remarks: Infrastructure upgrades currently underway

Potential Program R\&D Applications are Shaded

\begin{tabular}{|l|l|l|l|}
\hline Light Water Reactors & $\begin{array}{l}\text { Irradiated Fuel } \\
\text { Separations }\end{array}$ & $\begin{array}{l}\text { Advanced Fuel } \\
\text { Development }\end{array}$ & Space Power Systems \\
\hline $\begin{array}{l}\text { High-Temperature } \\
\text { Reactor }\end{array}$ & Fast Spectrum Reactor & $\begin{array}{l}\text { Safeguards and } \\
\text { Security }\end{array}$ & $\begin{array}{l}\text { Modeling and } \\
\text { Simulation }\end{array}$ \\
\hline
\end{tabular}

The TA-55 Plutonium Facility is a large, 230,000- $\mathrm{ft}^{2}$ nuclear facility within the Los Alamos National Laboratory Technical Area -55. It was constructed in the mid-1970s and began operation in 1978. Significant infrastructure upgrades to the facility are currently in progress.

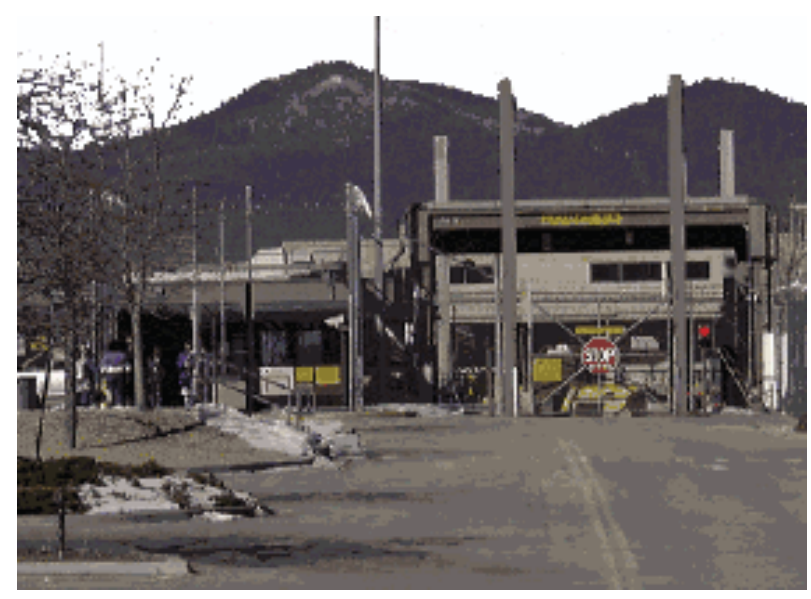

Entrance to the TA-55 Plutonium Facility at the Los Alamos National Laboratory

plutonium oxide) type fuel pellets. At research-scale quantities, this line is capable of working with fuels containing neptunium and small quantities of americium. The line also has been used to fabricate fuels for space reactors and MOX fuels in support of the surplus weapons plutonium disposition program.

The facility was designed and built to meet Security Category I and Hazard Category 2 requirements. It is primarily used for NNSA Defense Programs, but also hosts a broad portfolio of other programs. It has extensive, lightly shielded, inert glovebox capability and is designed with an integrated overhead trolley system for moving material between glovebox lines and to different parts of the building. These gloveboxes are capable of handling kilogram quantities of nuclear material as long as the associated radiation fields are relatively low.

Within the facility, there is a glovebox line (40 boxes) for fabrication of radioisotope heat sources and another series of connected glovebox functions as a development fuel line for ceramic fuels. This line is currently being used to fabricate research-scale quantities of MOX (mixed uranium 


\section{Transient Reactor Test (TREAT) Facility}

Location: Idaho National Laboratory

Currently Supporting: National and Homeland Security

Status: Standby

Remarks: Facility will need a comprehensive restart effort

\begin{tabular}{|l|l|l|l|}
\hline Potential Program R\&D Applications are Shaded \\
\hline Light Water Reactors & $\begin{array}{l}\text { Irradiated Fuel } \\
\text { Separations }\end{array}$ & $\begin{array}{l}\text { Advanced Fuel } \\
\text { Development }\end{array}$ & Space Power Systems \\
\hline $\begin{array}{l}\text { High-Temperature } \\
\text { Reactor }\end{array}$ & Fast Spectrum Reactor & $\begin{array}{l}\text { Safeguards and } \\
\text { Security }\end{array}$ & $\begin{array}{l}\text { Modeling and } \\
\text { Simulation }\end{array}$ \\
\hline
\end{tabular}

TREAT is an air-cooled, uranium-fueled, thermal-spectrum reactor constructed in 1958 and operated extensively in support of thermal and fast reactor development and safety testing from initial criticality in 1959 until placed in non-operational standby mode in 1994. It is located at the Materials and Fuels Complex of the Idaho National Laboratory.

The reactor and its control system were designed to expose reactor fuels (full-length elements and assemblies) and structural materials to conditions simulating various types of severe nuclear and thermal

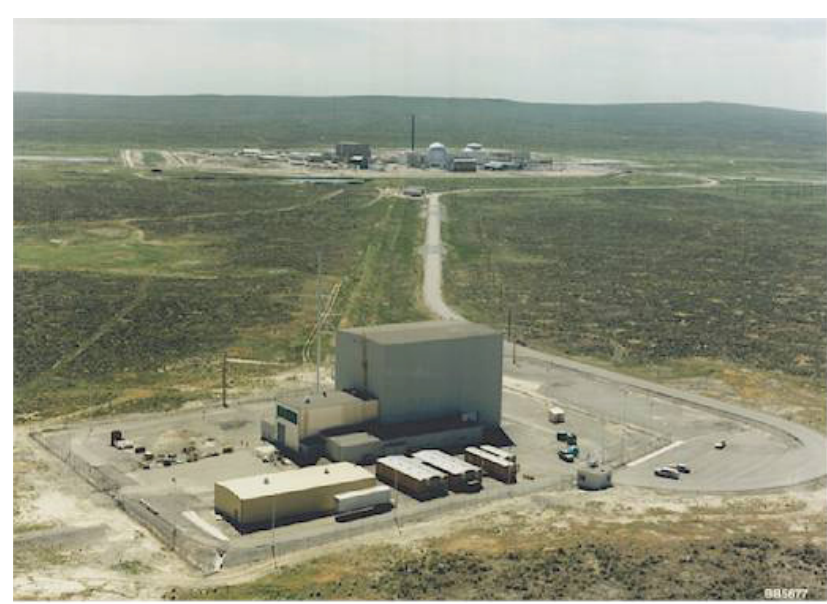

Transient Reactor Test Facility at the Idaho National Laboratory transient situations that could potentially occur in a nuclear reactor (e.g., TMI-II conditions). Fuel meltdowns, metal-water reactions, thermal interactions between overheated fuel and coolant, and the transient behavior of ceramic and metal fuel for high-temperature systems have been studied and could be studied again. Other tests have included the nuclear-driven laser experiment series and core-physics measurements. In its steady-state operation, TREAT can be used as a large neutron radiography facility.

Currently, the TREAT reactor is maintained in a safe shutdown configuration and provides support for out-of-core National and Homeland Security and other R\&D activities. 


\section{Unirradiated Fuel Storage Facility}

Location: Idaho National Laboratory

\section{Currently Supporting: Storage}

Status: Operational

Remarks: Ventilation system and security upgrades are required

\begin{tabular}{|l|l|l|l|}
\hline Potential Program R\&D Applications are Shaded \\
\hline Light Water Reactors & $\begin{array}{l}\text { Irradiated Fuel } \\
\text { Separations }\end{array}$ & $\begin{array}{l}\text { Advanced Fuel } \\
\text { Development }\end{array}$ & Space Power Systems \\
\hline $\begin{array}{l}\text { High-Temperature } \\
\text { Reactor }\end{array}$ & Fast Spectrum Reactor & $\begin{array}{l}\text { Safeguards and } \\
\text { Security }\end{array}$ & $\begin{array}{l}\text { Modeling and } \\
\text { Simulation }\end{array}$ \\
\hline
\end{tabular}

The Unirradiated Fuel Storage Facility is a fuel storage facility that provides secure storage of a variety of unirradiated fuel materials for subsequent processing or shipment to other facilities for use. Original construction was completed in 1975 and upgrades were completed in 1984 and 1994.

The facility consists of a south vault, north vault, receiving area, annulus on the north, east, and south sides of the vaults, and exterior concrete walls. On the north, east, and south sides of the building, there

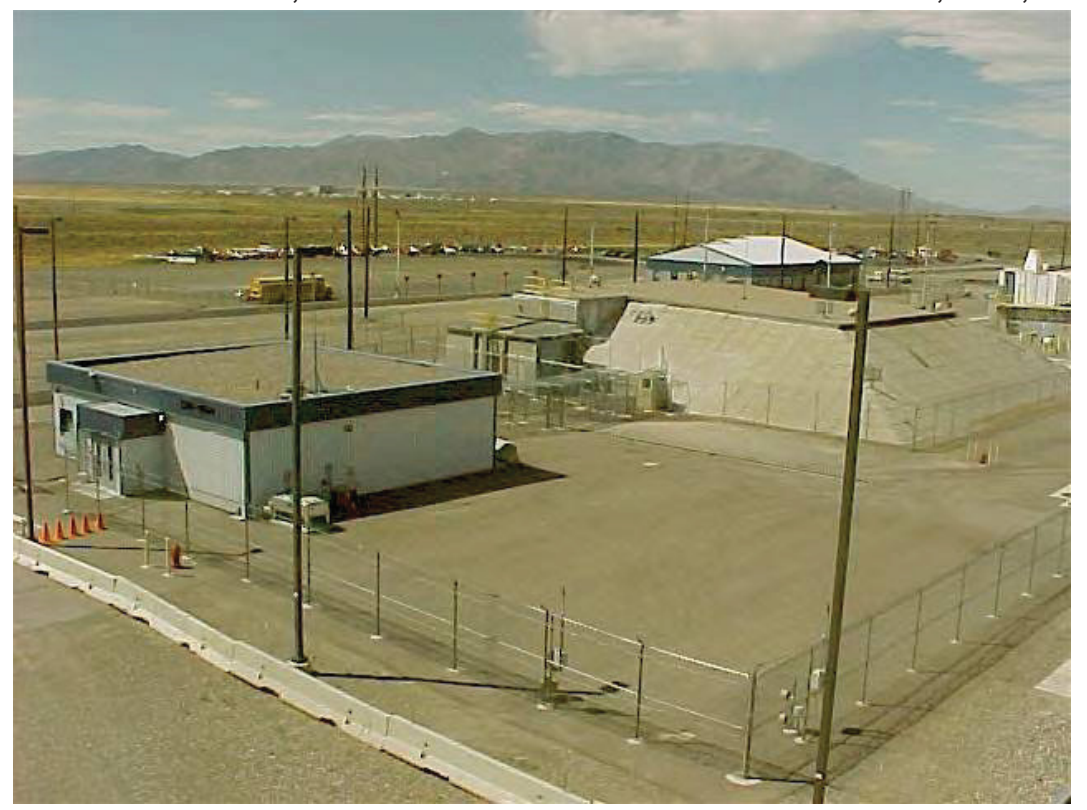

is a sloped berm from ground level to the roof. The inner core section of the berm consists of a rock fill material; compacted gravel fills the outer section and the berm is covered by non-structural concrete slabs.

The thick concrete walls and berm provide security and safeguards protection for stored fuel. Ventilation at the facility is poor and is only functional when personnel must enter it. Chain-link fences surround the facility and two sets of security doors (inner and outer doors) provide access.

The Unirradiated Fuel Storage Facility at the Idaho National Laboratory 


\section{Zero Power Physics Reactor}

Location: Idaho National Laboratory

Currently Supporting: Space and security missions and nuclear fuel handling and storage

Status: Reactor matrix scheduled for disposal; facility available for current and other missions

Remarks: Facility will need a comprehensive restart effort for reactor operation

\begin{tabular}{|l|l|l|l|}
\hline Potential Program R\&D Applications are Shaded \\
\hline Light Water Reactors & $\begin{array}{l}\text { Irradiated Fuel } \\
\text { Separations }\end{array}$ & $\begin{array}{l}\text { Advanced Fuel } \\
\text { Development }\end{array}$ & Space Power Systems \\
\hline $\begin{array}{l}\text { High-Temperature } \\
\text { Reactor }\end{array}$ & Fast Spectrum Reactor & $\begin{array}{l}\text { Safeguards and } \\
\text { Security }\end{array}$ & $\begin{array}{l}\text { Modeling and } \\
\text { Simulation }\end{array}$ \\
\hline
\end{tabular}

The Zero Power Physics Reactor, the nation's largest split-table critical facility, was designed and operated to model large reactor cores. The reactor is located within the Idaho National Laboratory's Materials and Fuels Complex property-protected area and is surrounded by a perimeter intrusion, detection, and assessment system.

It was constructed in the late 1960s and operated between 1969 and 1992. The design provided great flexibility in reactor core configuration and its operation provided a wealth of information needed for fast reactor design and operation. Core mockups included EBR-II, several large liquid metal fast breeder reactors, and space reactors. In 1992, all fissile material was removed from the core to the vault and the reactor was placed in non-operational standby.

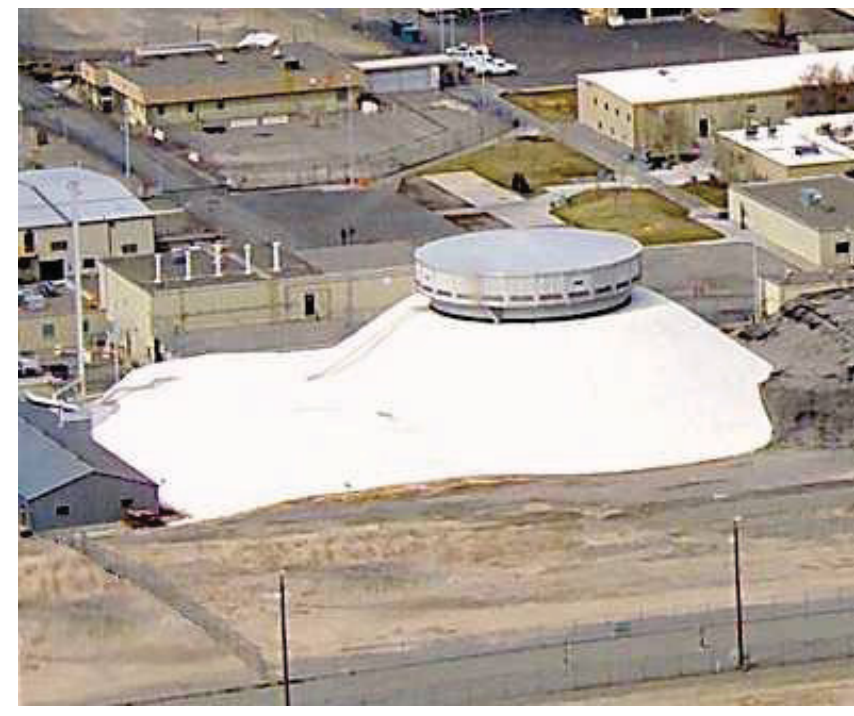

Zero Power Physics Reactor at the Idaho National Laboratory
Currently, unirradiated special nuclear materials are stored in the vault. The workroom is used to monitor and maintain the integrity of the stored materials and other areas are used to support various nuclear and non-nuclear programs.

Although disassembly and disposal of the reactor core matrix is planned, it is not funded and reactor operation could be restarted. If the core matrix is removed, it will free up significant space useful for a variety of nuclear programs, including those with high security requirements.

Restart of the Zero Power Physics Reactor as a critical facility has been estimated to cost $\$ 60 \mathrm{M}$. 


\section{Summary of Reviewer Comments}

1. There were numerous suggestions for modifications to descriptions of facilities included in this section. 


\subsubsection{U.S. Commercial Industry}

\section{B\&W Fuel Fabrication Facility}

Location: Babcock and Wilcox Company Nuclear Operations Group, Lynchburg, Virginia

Currently Supporting: Nuclear fuel and component supplier

Status: Operating

\begin{tabular}{|l|l|l|l|}
\hline Potential Program R\&D Applications are Shaded \\
\hline Light Water Reactors & $\begin{array}{l}\text { Irradiated Fuel } \\
\text { Separations }\end{array}$ & $\begin{array}{l}\text { Advanced Fuel } \\
\text { Development }\end{array}$ & Space Power Systems \\
\hline $\begin{array}{l}\text { High-Temperature } \\
\text { Reactor }\end{array}$ & Fast Spectrum Reactor & $\begin{array}{l}\text { Safeguards and } \\
\text { Security }\end{array}$ & $\begin{array}{l}\text { Modeling and } \\
\text { Simulation }\end{array}$ \\
\hline
\end{tabular}

The B\&W Nuclear Operations Group's (NOG) Lynchburg, Virginia facility is one of only two commercial plants licensed in the U.S. to possess, store, and characterize high-enriched uranium. This plant, regulated by the Nuclear Regulatory Commission and secured by a highly trained paramilitary force, accommodates the fabrication of nuclear fuel and precision components that range from a few grams to hundreds of tons. The site's in-house capabilities include advanced heat treatment to optimize components' material properties. The facility's

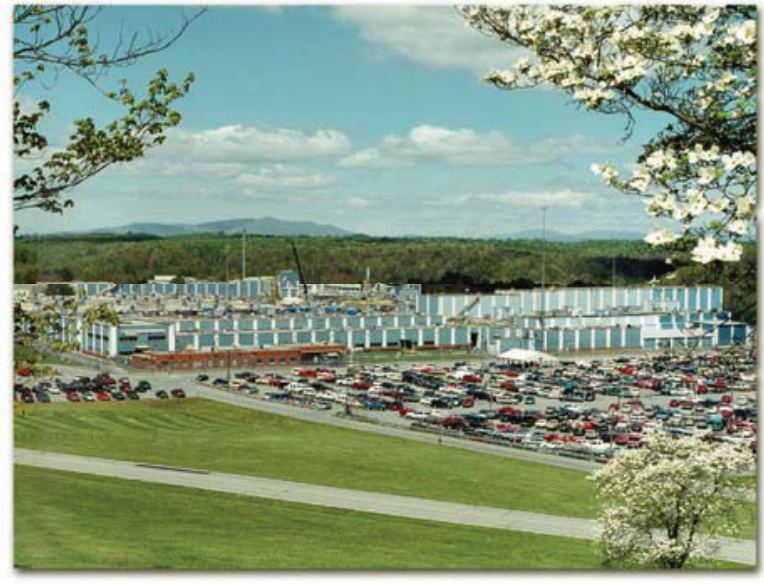

B\&W Lynchburg, Virginia facility controlled clean-room environment has the capacity to assemble railcar-size components.

In-depth quality control measures utilize leadingedge equipment and thorough testing, including destructive/nondestructive testing, computerized and real-time accept/reject dimensional inspection, custom inspection gauging and calibration, and ultrasonic, cryogenic, and dye penetrant inspections.

B\&W NOG's Lynchburg facility houses the group's Uranium Processing and Research Reactors group, which is the only North American supplier of research reactor fuel elements for colleges, universities, and national laboratories. The unit also produces and supplies uranium targets for medical isotopes, which are highly effective in detecting and treating specific types of cancer.

In addition, B\&W NOG's Mount Vernon and Barberton, Ohio locations are the only domestic suppliers of large, heavy-pressure vessels that have American Society of Mechanical Engineers N-Stamp accreditation. The Mount Vernon, Indiana facility is strategically placed on the Ohio River and provides total lift capability of 1,000 tons-the largest on the Ohio River.

The Euclid facility in Cleveland, Ohio is the most recent addition to the B\&W NOG family. B\&W NOG Euclid manufactures electromechanical equipment for U.S. government applications. 


\subsubsection{University}

\section{Advanced Materials Laboratory, Mechanical Testing Laboratory, Center for Materials Characterization and Spark-Plasma Sintering Unit}

Location: Boise State University, Boise, Idaho and Center for Advanced Energy Studies, Idaho Falls, Idaho

Currently Supporting: NERI, NGNP, Global Nuclear Energy Partnership, Nuclear Hydrogen Initiative

Status: Fully operational.

Remarks: Spark-plasma sintering unit will be moving from the Idaho Research Center to the Center for Advanced Energy Studies building in fall 2008.

\begin{tabular}{|l|l|l|l|}
\hline Potential Program R\&D Applications are Shaded \\
\hline Light Water Reactors & $\begin{array}{l}\text { Irradiated Fuel } \\
\text { Separations }\end{array}$ & $\begin{array}{l}\text { Advanced Fuel } \\
\text { Development }\end{array}$ & Space Power Systems \\
\hline $\begin{array}{l}\text { High-Temperature } \\
\text { Reactor }\end{array}$ & Fast Spectrum Reactor & $\begin{array}{l}\text { Safeguards and } \\
\text { Security }\end{array}$ & $\begin{array}{l}\text { Modeling and } \\
\text { Simulation }\end{array}$ \\
\hline
\end{tabular}

The Advanced Materials Laboratory is currently equipped with two inert atmosphere gloveboxes The Advanced Materials Laboratory also contains high-temperature box and tube furnaces including a hightemperature $\left(1700^{\circ} \mathrm{C}\right)$ controlled-atmosphere alumina tube furnace with a turbomolecular pump that allows for evacuation pressures as low as $10^{-6}$ Torr. Other Advanced Materials Laboratory equipment

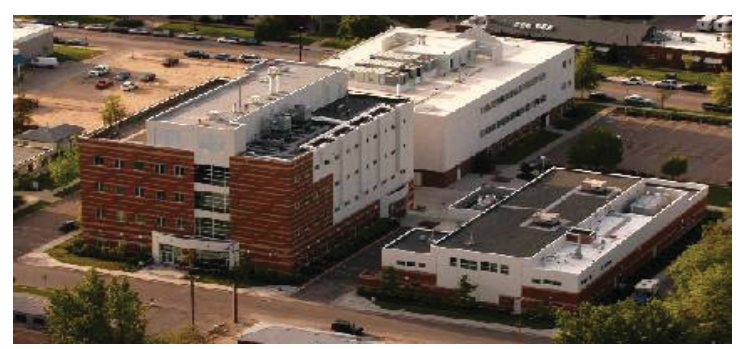

College of Engineering at Boise State University. includes a planetary ball mill (used with both radioactive and non-radioactive materials), an optical microscope, a laser particle size analyzer, a TGA/DSC system, and multiple potentiostats for electrochemical experiments. This laboratory maintains a Nuclear Regulatory Commission license.

A hydraulically-driven mechanical testing system is equipped for tension, compression, and 3 and 4-point bend testing at temperatures up to $1200^{\circ} \mathrm{C}$. The maximum load cell capacity is $100 \mathrm{kN}$ and is controlled with a FlexTest SE controller. A high-temperature extensometer allows for accurate measurement of displacement at temperatures up to $1200^{\circ} \mathrm{C}$.

The Boise State Center for Materials Characterization has a transmission electron microscope with a high-resolution pole piece. The transmission electron microscope has a point resolution of $0.23 \mathrm{~nm}$ and has energy dispersive $\mathrm{x}$-ray spectroscopy, tomography, and magnetic field neutralizing capabilities. The Boise State Center for Materials Characterization also has an X-ray diffractometer system for high-resolution determination of crystallographic structure, phase composition, and texture. This instrument includes the ability to run under non-ambient temperatures $\left(-180^{\circ} \mathrm{C}\right.$ to $\left.1600^{\circ} \mathrm{C}\right)$ and low-angle diffraction for thin film characterization.

A spark-plasma sintering system located at the Idaho Research Center (moving to Center for Advanced Energy Studies) is capable of sintering metals, ceramics, and advanced materials in very short times. The primary advantage of the spark-plasma sintering is to produce materials in short time (less than 1 hour) and produce materials that are difficult to fabricate by other means. The spark-plasma sintering unit is capable of applying forces between 5 and $50 \mathrm{kN}$, operating up to $2000^{\circ} \mathrm{C}$, and an output current of 1,500 A. Programmable temperature and pressure profiles are available. SEM (with EDS, EBSD, SE and BSE), AFM, and optical profilometry capabilities are also available at Boise State University. 


\section{Georgia Tech Nuclear and Radiological Engineering Facilities}

Location: Georgia Institute of Technology

Currently Supporting: Nuclear and Radiological Engineering and Medical Physics

Status: Fully Operational

Remarks: Facilities moving to a new location on campus soon. Additional capabilities will be added in the new location.

Potential Program R\&D Applications are Shaded

\begin{tabular}{|l|l|l|l|}
\hline Light Water Reactors & $\begin{array}{l}\text { Irradiated Fuel } \\
\text { Separations }\end{array}$ & $\begin{array}{l}\text { Advanced Fuel } \\
\text { Development }\end{array}$ & Space Power Systems \\
\hline $\begin{array}{l}\text { High-Temperature } \\
\text { Reactor }\end{array}$ & Fast Spectrum Reactor & $\begin{array}{l}\text { Safeguards and } \\
\text { Security }\end{array}$ & $\begin{array}{l}\text { Modeling and } \\
\text { Simulation }\end{array}$ \\
\hline
\end{tabular}

High-Performance Computing Clusters: The NRE Program currently has 13 computer clusters ranging from 8 to 168 and totaling over 1,000 processors. The clusters are used in modeling, simulation, and the development of radiation transport codes. Across the computer clusters many common NE computer codes used in radiation transport, criticality, and shielding and dosimetry are installed.

Neutron Reference Field Laboratory: This neutron laboratory is used to produce reference neutron fields for dosimetry studies and instrument development and calibrations. Reference fields available include; unmoderated Cf-252, heavy-water-moderated Cf-252, polyethylene-moderated Cf-252, and iron

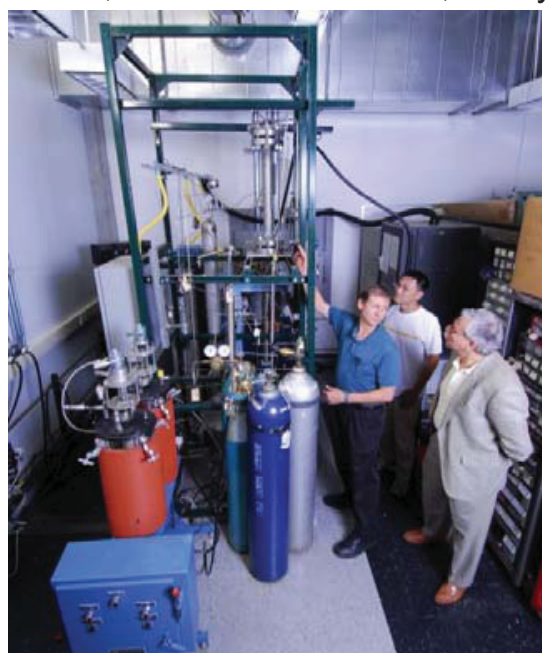

PWR Axial Offset Anomaly Test Facility and lead moderators for use with the Cf-252 and additional $\mathrm{PuBe}$ and $\mathrm{AmBe}$ sources. A small portable neutron generator capable of producing $108 \mathrm{n} / \mathrm{sec}$ will be added to the source capabilities in fall 2009. Also available are a NE 213 spectrometer, a Bonner sphere spectrometer system, and numerous activation foils for measuring the neutron spectra.

Microchannel Test Facility: Facility simulates single and two-phase phenomena in high heat flux systems such as accelerator targets, compact fission reactor cores and fusion first walls. Among the phenomena investigated are: two-phase flow instability, critical heat flux, single-phase forced convection, twophase pressure drop, and two-phase flow regimes.

PWR Axial Offset Anomaly Test Facility: Test loop designed to operate at prototypical PWR primary loop conditions. Facility simulates conditions corresponding to crud deposition and boron precipitation on fuel rods and examines various practical solutions aimed at preventing Axial Offset Anomaly.

Neutron Generator Facility: Layout and design of a neutron generator facility based off a D-D or D-T neutron generator capable of producing up to $1011 \mathrm{n} / \mathrm{sec}$ is currently being developed for installation in the new campus location of the NRE program. The neutron generator for the facility has both head and tail pulsing capabilities resulting in very low neutron production between pulses. 


\section{Idaho State University Accelerator Center and Nuclear Engineering Laboratory}

Location: Idaho State University, Pocatello, Idaho

Currently Supporting: Student research, undergraduate, and graduate laboratory courses and workshops in nuclear science experiments and operations

Status: Fully operational facilities in four major laboratories, including one operating reactor and 11 operating accelerators

\begin{tabular}{|l|l|l|l|}
\hline Potential Program R\&D Applications are Shaded \\
\hline Light Water Reactors & $\begin{array}{l}\text { Irradiated Fuel } \\
\text { Separations }\end{array}$ & $\begin{array}{l}\text { Advanced Fuel } \\
\text { Development }\end{array}$ & Space Power Systems \\
\hline $\begin{array}{l}\text { High-Temperature } \\
\text { Reactor }\end{array}$ & Fast Spectrum Reactor & $\begin{array}{l}\text { Safeguards and } \\
\text { Security }\end{array}$ & $\begin{array}{l}\text { Modeling and } \\
\text { Simulation }\end{array}$ \\
\hline
\end{tabular}
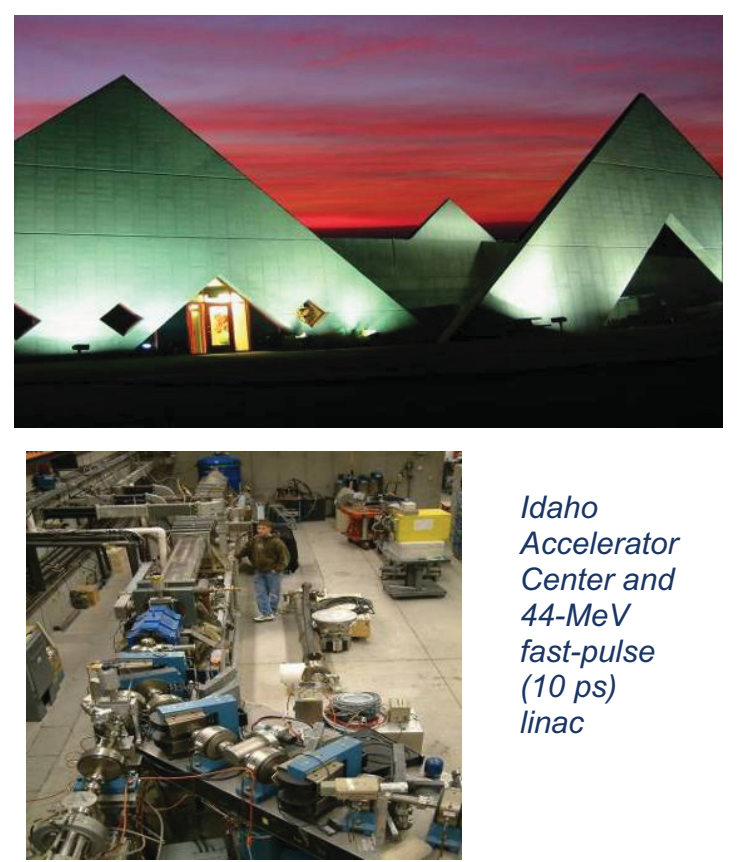

The Idaho Accelerator Center is a multiaccelerator applied physics laboratory dedicated to research and education in non-proliferation, instrumentation and detector development and testing, fuel-cycle materials, homeland security, nuclear physics, health physics, pulsed power, medical physics and related topics. The center operates electron linacs ranging from 2 to 44 $\mathrm{MeV}$, pulsed-power electron sources (up to $8 \mathrm{MeV}, 10$ $\mathrm{kA}$ ) and a positive ion Van de Graaff (2MV). The Idaho Accelerator Center supports over 60 students of nuclear science and engineering (B.S. through Ph.D.). The Idaho Accelerator Center also has major collaborations with seven national laboratories, including Idaho, Pacific Northwest, Los Alamos, Lawrence Livermore, Oak Ridge, Sandia, and Savannah River.

The Nuclear Engineering Laboratory is the principal laboratory used by Idaho State University Nuclear Engineering Program students to gain direct, hands-on operating experience working with nuclear materials, machines, and measuring apparatus. It is comprised of the Idaho State University AGN-201M Nuclear Reactor

Laboratory and the Subcritical Assembly Facility. The reactor, licensed to operate at $5 \mathrm{~W}$, has been in continuous operation at Idaho State University since 1967. The reactor is self-contained and has experimental access ports that allow small samples and neutron counters to be inserted into the center of the reactor core for activation and flux measurements. A 20-year operating license extension was approved by the Nuclear Regulatory Commission in 2006 and a control console replacement was completed in 2008. Application for 10-year extension of materials license for the Subcritical Assembly was submitted in 2008. These laboratories play an integral part in nuclear science and engineering education by providing direct experimental experiences that verify and demonstrate theoretical models. Experiments range from detector development to reactor operating characteristics and from nuclear physics to 'approaches to criticality'. In addition, these laboratories are supported by the Department of Engineering's Radiation Metrology Laboratory; by the Department of Physics' two radiation measurements laboratories and a detector development laboratory; and by the abundant radiation measurement instrumentation of the Idaho Accelerator Center. 


\title{
Kansas State University TRIGA Mark II Reactor and Semiconductor Materials and Radiation Technologies Laboratory
}

\author{
Location: Kansas State University
}

Currently Supporting: Multiple programs

Status: Operational

Potential Program R\&D Applications are Shaded

\begin{tabular}{|l|l|l|l|}
\hline Light Water Reactors & $\begin{array}{l}\text { Irradiated Fuel } \\
\text { Separations }\end{array}$ & $\begin{array}{l}\text { Advanced Fuel } \\
\text { Development }\end{array}$ & Space Power Systems \\
\hline $\begin{array}{l}\text { High-Temperature } \\
\text { Reactor }\end{array}$ & Fast Spectrum Reactor & $\begin{array}{l}\text { Safeguards and } \\
\text { Security }\end{array}$ & $\begin{array}{l}\text { Modeling and } \\
\text { Simulation }\end{array}$ \\
\hline
\end{tabular}

The Kansas State reactor has been in continuous operation since 1962, and is currently licensed to $1.25 \mathrm{MW}$ with pulsing to greater than 1,000 MW. The reactor was relicensed March 2008 for an additional 20 years of service. The Semiconductor Materials and Radiation Technologies Laboratory was established in 1997 and supports research and development of new and innovative radiation detector technologies.

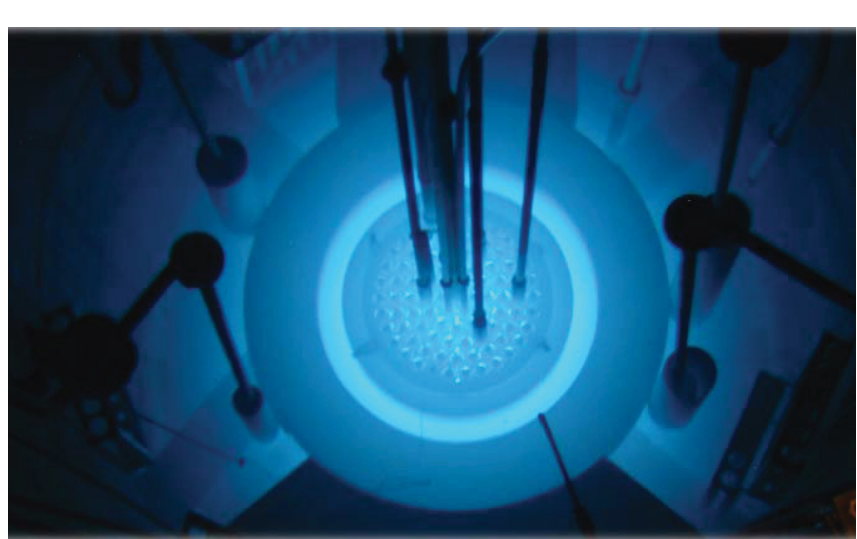

Kansas State University TRIGA Mark II Reactor Core
The Kansas State University TRIGA Mark II reactor supports training, research, and isotope production. The facility is integrated in Kansas State University nuclear education, and supports commercial reactor operator training. Research is supported through gamma irradiation, thermal and fast neutron irradiation, neutron activation analysis, and neutron beams (detector testing and neutron scattering, radiography, and transmission).

Irradiation sites include a central thimble, pneumatic tube, radial reflector space, and space outside the reflector. One beam port is tangent to and one pierces the reflector. Two ports are radial to the core. Two thermal

columns support large irradiations. Steady state reactor power is variable up to $1.25 \mathrm{MW}$, with pulsing capability. Peak neutron flux is 4E10 thermal and 4.8E10 fast $(>10 \mathrm{keV})$ neutrons per $\mathrm{cm}^{2}$ per $\mathrm{kW}$-second, with 100 rad per $\mathrm{kW}$-second gamma. The pneumatic tube provides $43 \%$ of peak thermal flux, $53 \%$ of peak fast flux. The reflector site provides $18 \%$ of peak thermal flux, $13 \%$ of peak fast flux in a larger space. The piercing beam port provides $20 \%$ of peak thermal flux and $17 \%$ of peak fast flux. Gamma irradiation with the reactor shutdown is conducted in the central thimble (1.33 in. diameter) or outside the reflector.

The Semiconductor Materials and Radiation Technologies Laboratory develops, investigates, and fabricates a variety of detectors, including compact low-power neutron detectors, high-resolution roomtemperature-operated semiconductor gamma ray spectrometers, pixelated devices (gamma ray or neutron imaging), miniaturized gas-filled detectors for general radiation and spectroscopic, homeland security, national laboratories, commercial nuclear reactor applications. 


\section{Massachusetts Institute of Technology Reactor (MITR)}

Location: Massachusetts Institute of Technology

Currently Supporting: Fuels and materials development, neutron science, isotope production (research quantities), nuclear education

Status: Operating

Remarks: Being relicensed for operation at $6 \mathrm{MW}$; design study ongoing for low-enriched uranium core conversion

Potential Program R\&D Applications are Shaded

\begin{tabular}{|l|l|l|l|}
\hline Light Water Reactors & $\begin{array}{l}\text { Irradiated Fuel } \\
\text { Separations }\end{array}$ & $\begin{array}{l}\text { Advanced Fuel } \\
\text { Development }\end{array}$ & Space Power Systems \\
\hline $\begin{array}{l}\text { High-Temperature } \\
\text { Reactor }\end{array}$ & Fast Spectrum Reactor & $\begin{array}{l}\text { Safeguards and } \\
\text { Security }\end{array}$ & $\begin{array}{l}\text { Modeling and } \\
\text { Simulation }\end{array}$ \\
\hline
\end{tabular}

The MITR is a tank-type water cooled research reactor. It is owned and operated by the Massachusetts Institute of Technology and is licensed by the Nuclear Regulatory Commission. The reactor has two tanks: an inner one for light water coolant/moderator and an outer one for heavy water as a reflector. A graphite reflector surrounds the heavy water tank. The light-water core, heavy-water reflector, and graphite region are all separately cooled.

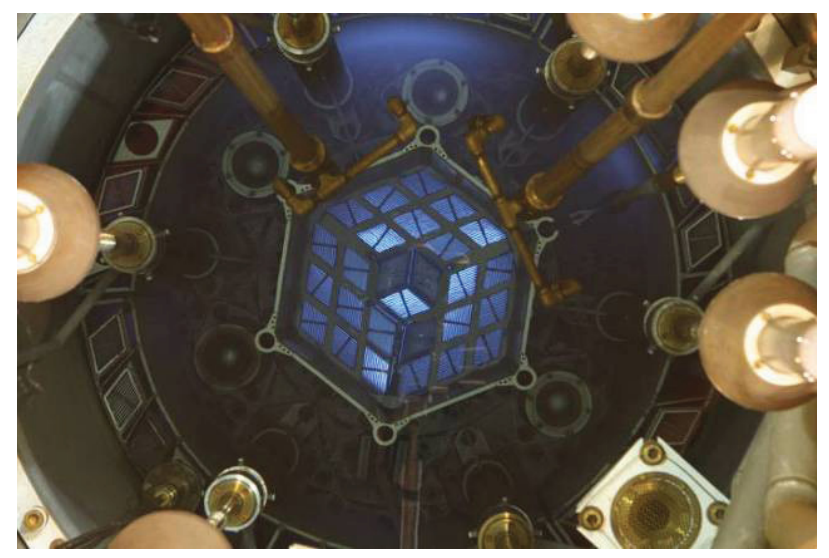

The compact MITR core has fast and thermal neutron fluxes similar to those of a commercial LWR
The reactor utilizes flat, plate-type fuel elements of $\mathrm{UAl}_{\mathrm{x}}$ cermet with 6061 aluminum alloy cladding. The core has 27 fuel element positions and is normally configured with 24 fuel elements; making 3 positions available for in-core experiments. The close-packed hexagonal core design maximizes the thermal neutron flux in the heavy water reflector region where the re-entrant thimbles of the beam ports are located. The MITR is equipped with a wide variety of sample irradiation facilities, beam ports and a pneumatic sample transfer system.

The MITR operates at atmospheric pressure. The primary coolant core inlet temperature is approximately $42^{\circ} \mathrm{C}$ and outlet temperature is about $50^{\circ} \mathrm{C}$. The hexagonal core structure is about $38 \mathrm{~cm}$ across with an active fuel length of about $56 \mathrm{~cm}$ with fast, thermal, and gamma fluxes similar to

those of a commercial LWR. The MITR operates 24 hours a day, 7 days per week and supports multiple research programs. A typical fuel cycle lasts about six weeks followed by a one week refueling and maintenance outage.

The MITR has an active in-core experiment program to support materials and fuel development for advanced reactors. Each in-core irradiation facility can generally be tailored to meet the requirements of a particular experiment or multiple experiments/specimens. Examples of previous experiments include pressurized loops for studies of BWR and PWR coolant chemistry; investigation of shadow corrosion and irradiation assisted stress corrosion cracking; advanced cladding development; a scoping study of advanced LWR fuel; and a high-temperature gas reactor materials irradiation study in inert gas up to $1600^{\circ} \mathrm{C}$. 


\section{PULSTAR Reactor}

Location: North Carolina State University, Raleigh, NC

Currently Supporting: Multiple programs

Status: Fully operational

Remarks: PULSTAR reactor is equipped with unique facilities for materials nondestructive examination

\begin{tabular}{|l|l|l|l|}
\hline Potential Program R\&D Applications are Shaded \\
\hline Light Water Reactors & $\begin{array}{l}\text { Irradiated Fuel } \\
\text { Separations }\end{array}$ & $\begin{array}{l}\text { Advanced Fuel } \\
\text { Development }\end{array}$ & Space Power Systems \\
\hline $\begin{array}{l}\text { High-Temperature } \\
\text { Reactor }\end{array}$ & Fast Spectrum Reactor & $\begin{array}{l}\text { Safeguards and } \\
\text { Security }\end{array}$ & $\begin{array}{l}\text { Modeling and } \\
\text { Simulation }\end{array}$ \\
\hline
\end{tabular}

The PULSTAR reactor has been operating since 1972. A major renovation and upgrade plan was initiated in 2002 and continues until the present date. This plan has resulted in the development of major experimental facilities for the support of fundamental and applied research. This includes the nation's only intense slow positron beam for materials defect analysis, a state-of-the-art neutron imaging facility, and a neutron powder diffraction system for materials structure analysis. These facilities are capable of performing nondestructive examination of materials at the microscopic and macroscopic scales. Currently, work is on going to develop an ultra-cold neutron source that is expected to match international systems in intensity and versatility.

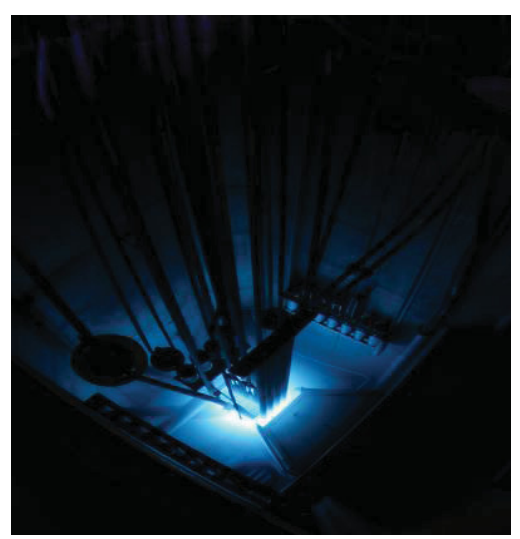

The PULSTAR Reactor at North Carolina State University
The PULSTAR reactor is a 1-MWth open pool type that is light water moderated and cooled. The core has dimensions of $15 \times 18 \times 24$ in. and is located in a pool that is 26 feet deep and is directly viewed by five beam tubes. Four of the beam tubes are circular and have diameters of 6 and 8 inches. One beam tube is square and has a cross section of $11.5 \times 11.5 \mathrm{in}$. A sixth beam tube represents a through tube that passes above the core from one side of the pool to the other.

The core of the PULSTAR reactor is fueled with uranium dioxide $\left(\mathrm{UO}_{2}\right)$ enriched to $4 \%$ in $\mathrm{U}-235$. The fuel is located in zircaloy fuel assemblies that are configured in a $5 \times 5$ grid. Each fuel assembly is composed of 25 fuel rods that are also configured in a $5 \times 5$ array. The fuel rods are composed of pellets that are stacked in tubes of zircaloy cladding. The core is reflected by a set of beryllium reflectors on one side and graphite reflectors on another side. Control of the core is achieved using four plate-type control rods that are made of an Ag-In-Cd alloy.

A significant operational advantage of the PULSTAR is that its thermal neutron flux peaks at the interface between the core and the reflectors. Therefore, the peak thermal flux will occur near the entrance of the beam tubes facing the core. The level of the flux at such locations has been measured and was found to be around $2 \times 10^{13} \mathrm{n} / \mathrm{cm}^{2} \cdot \mathrm{s}$. These levels of neutron flux indicate that the PULSTAR core design facilitates the leakage of core neutrons into the beam tubes and eventually to experimental stations. Consequently, the PULSTAR experimental facilities have benefited from enhanced neutron intensities that are typical of higher power reactors. 


\section{Ohio State University Nuclear Reactor Laboratory}

Location: The Ohio State University

Currently Supporting: DOE-SBIR projects with industrial partners

Status: In full operation

Remarks: Has supported a number of NERI development projects

\begin{tabular}{|l|l|l|l|}
\hline \multicolumn{2}{|l|}{ Potential Program R\&D Applications are Shaded } \\
\hline Light Water Reactors & $\begin{array}{l}\text { Irradiated Fuel } \\
\text { Separations }\end{array}$ & $\begin{array}{l}\text { Advanced Fuel } \\
\text { Development }\end{array}$ & Space Power Systems \\
\hline $\begin{array}{l}\text { High-Temperature } \\
\text { Reactor }\end{array}$ & Fast Spectrum Reactor & $\begin{array}{l}\text { Safeguards and } \\
\text { Security }\end{array}$ & $\begin{array}{l}\text { Modeling and } \\
\text { Simulation }\end{array}$ \\
\hline
\end{tabular}

The principal experimental facility at the Ohio State University Nuclear Reactor Laboratory is the Ohio State University Research Reactor. The Ohio State University Research Reactor is a pool-type reactor that is utilized for a variety of instructional, research, and service activities. It is licensed to operate at continuously variable thermal power up to a maximum of 500 kilowatts. At the maximum steady-state power, the average thermal neutron flux in the core is approximately $5 \times 10^{12} \mathrm{n} / \mathrm{cm}^{2} / \mathrm{s}$. The reactor is immersed in a pool of light water that provides moderation and cooling by natural convective flow. It was constructed in 1961 and subsequently modified to operate with low-enriched fuel.

A number of experimental facilities converge at the reactor core, which allows simultaneous performance of multiple experiments. These facilities include two beam ports, a pneumatic transfer facility, a graphite thermal column, a central irradiation facility that extends into a water-filled flux trap, an auxiliary irradiation facility, and multiple movable dry tubes. The central irradiation facility consists of a 1.3-inch inner-

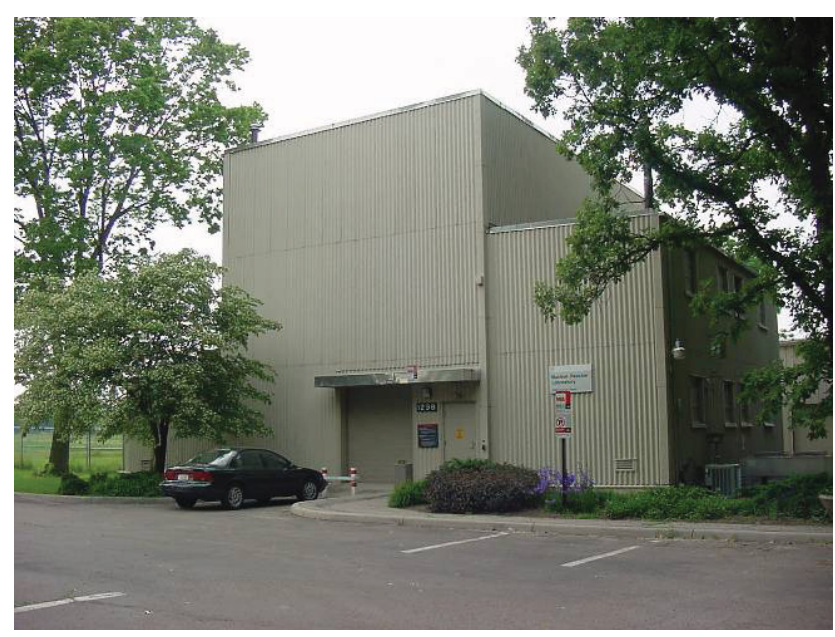

The Nuclear Reactor Laboratory at Ohio State University diameter tube that extends from the top of the reactor pool down into the central grid position of the core. This facility has the highest available flux at the Ohio State University Research Reactor, with a maximum total flux of $3 \times 10^{13} \mathrm{n} / \mathrm{cm}^{2} / \mathrm{s}$, and a maximum thermal flux of $1.5 \times 10^{13} \mathrm{n} / \mathrm{cm}^{2} / \mathrm{s}$.

The Ohio State University Research Reactor is used for a wide range of research endeavors, including neutron activation analysis, radiationdamage evaluation for materials and electronic components, development and testing of neutron and radiation sensitive detectors, isotope production, and biomedical experiments. Service irradiations are also provided to industrial clients for qualification testing of components. 


\section{Oregon State University}

Location: Radiation Center and the Department of Nuclear Engineering and Radiation Health Physics

Currently Supporting: Multiple programs including Advanced Fuel Cycle Initiative and Global Nuclear Energy Partnership

Status: Fully operational

Remarks: Low-enriched uranium fuel conversion of reactor to be completed in the fall of 2008

\begin{tabular}{|l|l|l|l|}
\hline Potential Program R\&D Applications are Shaded \\
\hline Light Water Reactors & $\begin{array}{l}\text { Irradiated Fuel } \\
\text { Separations }\end{array}$ & $\begin{array}{l}\text { Advanced Fuel } \\
\text { Development }\end{array}$ & Space Power Systems \\
\hline $\begin{array}{l}\text { High-Temperature } \\
\text { Reactor }\end{array}$ & Fast Spectrum Reactor & $\begin{array}{l}\text { Safeguards and } \\
\text { Security }\end{array}$ & $\begin{array}{l}\text { Modeling and } \\
\text { Simulation }\end{array}$ \\
\hline
\end{tabular}

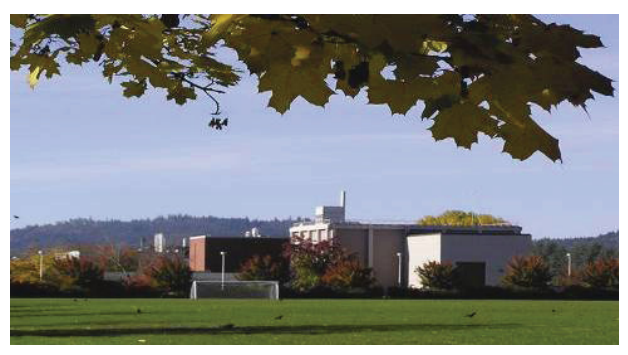

Oregon State University Radiation Center

The Oregon State University Radiation Center and the Department of Nuclear Engineering and Radiation Health Physics jointly offer unique research capabilities within a facility with specialized amenities for nuclear-related work. These include: a research reactor; a gamma irradiator; laboratories for radiochemical, radioecological, engineering, and computational research; and, faculty and staff with the capability to design, manufacture, model and test a variety of nuclear-related systems. The $1 \mathrm{MW}$ TRIGA Mark II reactor is licensed to operate continuously at up to $1.1 \mathrm{MW}$ and pulsed to $2500 \mathrm{MW}$. It has multiple irradiation facilities in- and out- of core for performing neutron activation and radioelement analyses; and includes beam ports and a neutron radiography facility capable of imaging large structures.

The one-quarter scale thermal hydraulic Advanced Plant Experimental test facility models all thermal hydraulic phenomena of the Westinghouse AP600 and AP1000 reactor designs and has been used in safety evaluation and licensing for the full scale plant. This reconfigurable facility is 10 CFR 50.59 compliant.

The Advanced Thermal Hydraulic Research Laboratory investigates fundamental processes in multiphase fluid flow and heat transfer. The Advanced Thermal Hydraulic Research Laboratory meets NQA-1 and 10 CFR 50 Appendix B quality assurance requirements for certification of instrument calibration. It has advanced multi-phase flow calculation capabilities and a professional design, construction and test operations team.

The Laboratory of Transuranic Elements is a state-of-the-art research laboratory focused on speciation chemistry of actinides and fission products in aqueous and organic matrices for application in advanced separation processes, development of new materials, and environmental monitoring. Highly specialized spectroscopic instrumentation is applied for quantification of actinides and fission products and thermodynamic and kinetic studies of separation processes.

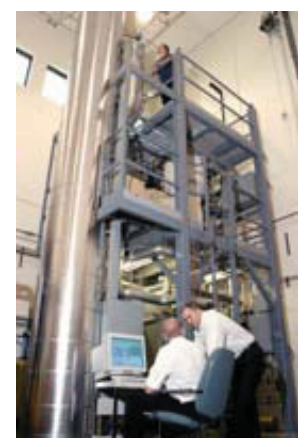

APEX test facility

The Advanced Nuclear Instrumentation Development Laboratory includes faculty and staff actively engaged in research and development activities in traditional detector design, hybrid design, light capture techniques, visible photon detection methods, neural network development, GUI development, and digital signal processing design. 


\section{Penn State Breazeale Reactor}

Location: Pennsylvania State University, Radiation Science and Engineering Center

Currently Supporting: Multiple programs, including nuclear engineering program research and courses

Status: Fully operational

Remarks: 1-MW TRIGA, Mark III Reactor (Penn State Breazeale Reactor). Third License renewal application was submitted in 2005. Various upgrades of experimental facilities including new radiochemistry program are in progress.

Potential Program R\&D Applications are Shaded

\begin{tabular}{|l|l|l|l|}
\hline Light Water Reactors & $\begin{array}{l}\text { Irradiated Fuel } \\
\text { Separations }\end{array}$ & $\begin{array}{l}\text { Advanced Fuel } \\
\text { Development }\end{array}$ & Space Power Systems \\
\hline $\begin{array}{l}\text { High-Temperature } \\
\text { Reactor }\end{array}$ & Fast Spectrum Reactor & $\begin{array}{l}\text { Safeguards and } \\
\text { Security }\end{array}$ & $\begin{array}{l}\text { Modeling and } \\
\text { Simulation }\end{array}$ \\
\hline
\end{tabular}

The Radiation Science and Engineering Center facilities include the Penn State Breazeale Reactor, gamma irradiation facilities (In-pool Irradiator, Dry Irradiator, and Hot Cells), and various radiation detection and measurement laboratories. The Penn State Breazeale Reactor, which first went critical in 1955 , is the nation's longest continuously operating university research reactor. The Penn State Breazeale Reactor is a $1 \mathrm{MW}$, TRIGA with pulsing capabilities. The core is moveable core within a $24 \mathrm{ft}$ deep pool with $\sim 71,000$ gallons of demineralized water.

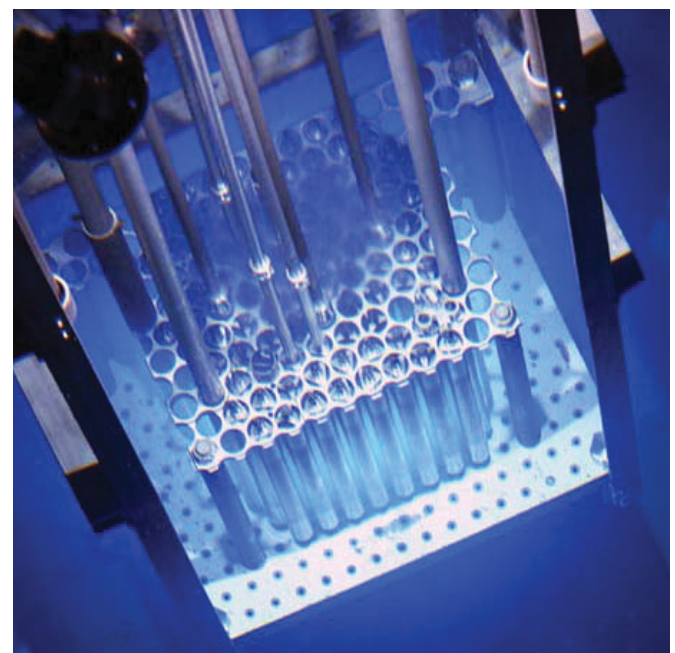

Core picture of Penn State Breazeale Reactor at Radiation Science and Engineering Center
A variety of dry tubes and fixtures are available in or near core irradiations. A pneumatic transfer system is also available for irradiation of samples. When the reactor core is placed next to a $\mathrm{D}_{2} \mathrm{O}$ tank and graphite reflector assembly near the beam port locations, thermal neutron beams become available for neutron transmission and neutron radiography measurement from two of the seven existing beam ports. In steady-state operation at $1 \mathrm{MW}$, the thermal neutron flux is $1 \times 10^{13} \mathrm{n} / \mathrm{cm}^{2} \mathrm{sec}$ at the edge of the core and $3 \times 10^{13} \mathrm{n} / \mathrm{cm}^{2} \mathrm{sec}$ at the central thimble. The Penn State Breazeale Reactor can also pulse with the peak flux for maximum pulse of about $6 \times 10^{16} \mathrm{n} / \mathrm{cm}^{2} \mathrm{sec}$ with pulse half width of about $10 \mathrm{msec}$.

The improvements for some existing Radiation Science and Engineering Center facilities are currently underway or just completed. New core-moderator and beam port arrangement are planned with an expansion of the existing beam laboratory. The primary research areas envisioned for Radiation Science and Engineering Center new beam port/beam hall design will be in cutting-edge nuclear science and materials science. Examples include the following: a Neutron Depth Profiling facility for depth vs. concentration measurements in materials; a Neutron Imaging facility that includes neutron computed tomography capabilities for imaging of fuel cells and other technologically important components; a Cold Neutron Source and Cold Neutron Prompt Gamma Activation Analysis for neutron focusing research and determination of impurities in historically or technologically important material; and a Neutron Powder Diffractometer for structural determination of materials. 


\section{PUMA and Reactor Safety Facility of the Thermal-hydraulics Reactor Safety Laboratory}

Location: Purdue University

Currently Supporting: Multiple programs

Status: Fully operational

Remarks: Project extension request of Purdue University Multi-dimensional Test Assembly to the Nuclear Regulatory Commission is in progress.

\begin{tabular}{|l|l|l|l|}
\hline Potential Program R\&D Applications are Shaded \\
\hline Light Water Reactors & $\begin{array}{l}\text { Irradiated Fuel } \\
\text { Separations }\end{array}$ & $\begin{array}{l}\text { Advanced Fuel } \\
\text { Development }\end{array}$ & Space Power Systems \\
\hline $\begin{array}{l}\text { High-Temperature } \\
\text { Reactor }\end{array}$ & Fast Spectrum Reactor & $\begin{array}{l}\text { Safeguards and } \\
\text { Security }\end{array}$ & $\begin{array}{l}\text { Modeling and } \\
\text { Simulation }\end{array}$ \\
\hline
\end{tabular}

Purdue University Multi-dimensional Test Assembly is a test facility designed to perform integral and separate effect tests, especially to assess the performance of natural circulation boiling water reactors with passive safety systems.

PUMA has been operating continuously since 1996, but because its internal components are periodically changed out, it remains a valuable research and test machine capable of several years more service. It was established as a SBWR Integral Test Facility by NRC in 1996.

The Reactor Safety Facility of Thermal-hydraulics Reactor Safety Laboratory at Purdue University has been supporting a wide range of fundamental reactor safety investigations. Some highlights of existing experimental capabilities are:

- 8 x 8 rod bundle loop (up to 10 bars pressure)

- Nuclear coupled two-phase flow instability loop (up to 10 bars)

- Suppression pool separate-effects loop

- Boiling loop simulating reactor core two-phase flow characteristics (up to 10 bars)

- Freon 113 two-phase flow loop simulating post dry out phenomena (up to critical point)

- Microgravity flow simulation loop

- Other two-phase flow testing facility (various channel geometry, size and flow orientation)

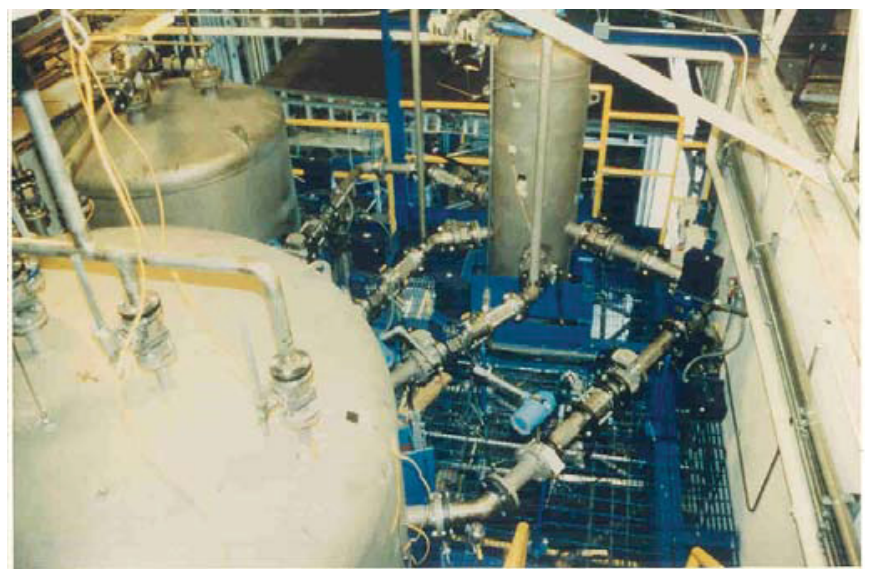

PUMA Facility at Purdue University
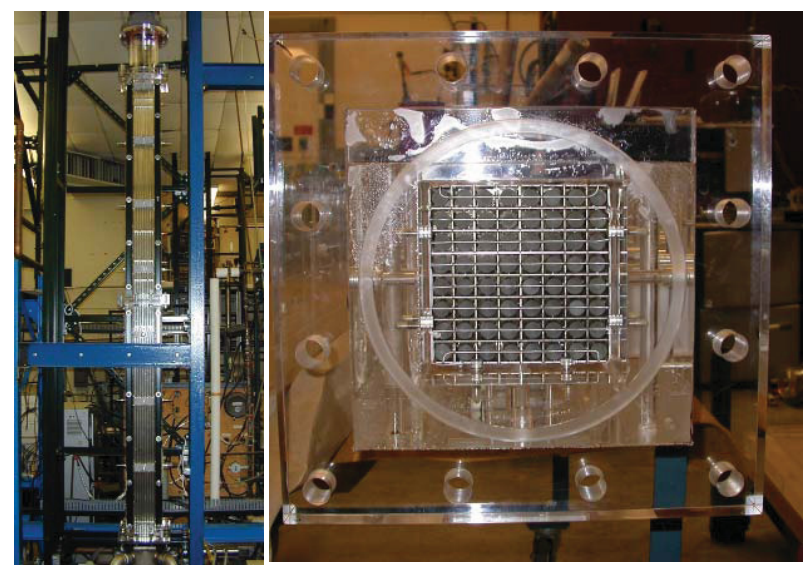

8 x 8 Rod Bundle Facility at Purdue University 


\section{Rensselaer Polytechnic Institute Gaerttner LINAC Laboratory and Critical Experiments Facility}

Location: Rensselaer Polytechnic Institute, Troy, New York

Currently Supporting: Multiple programs

Status: Both facilities are fully operational, with the Critical Experiments Facility operational to 15 watts

\begin{tabular}{|l|l|l|l|}
\hline Potential Program R\&D Applications are Shaded \\
\hline Light Water Reactors & $\begin{array}{l}\text { Irradiated Fuel } \\
\text { Separations }\end{array}$ & $\begin{array}{l}\text { Advanced Fuel } \\
\text { Development }\end{array}$ & Space Power Systems \\
\hline $\begin{array}{l}\text { High-Temperature } \\
\text { Reactor }\end{array}$ & Fast Spectrum Reactor & $\begin{array}{l}\text { Safeguards and } \\
\text { Security }\end{array}$ & $\begin{array}{l}\text { Modeling and } \\
\text { Simulation }\end{array}$ \\
\hline
\end{tabular}

The Gaerttner LINAC Laboratory located at Rensselaer Polytechninc Institute in Troy, NY has been engaged in active research continuously for over 45 years. Current areas of research at the LINAC include thermal reactor physics, photoneutron reactions, neutron cross sections, radiation effects in electronics, and production of medical isotopes. This laboratory has served government and industry in numerous applications where it has provided a unique and highly intense radiation environment. The Gaerttner LINAC Laboratory has been designated as a Nuclear Historic Landmark by the American Nuclear Society.

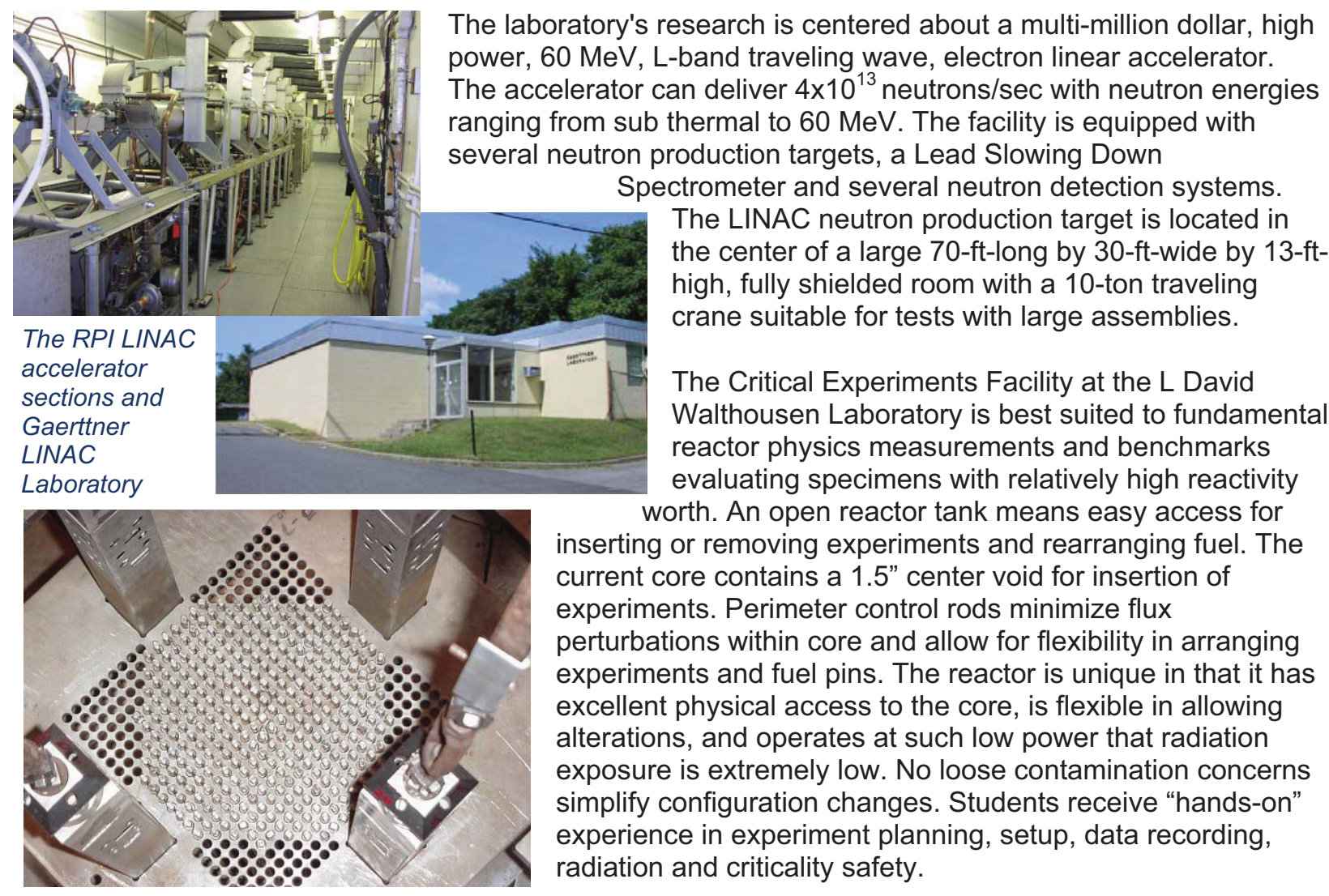

The Critical Experiments Facility reactor core (top view) 


\section{Texas A\&M University Fuel and Radiation Laboratories and Nuclear Science Center}

Location: Texas A\&M University

Currently Supporting: Multiple applied training and research programs for DOE, industry, and private foundations

Status: Fully operational

\begin{tabular}{|l|l|l|l|}
\hline Potential Program R\&D Aplications are Shaded \\
\hline Light Water Reactors & $\begin{array}{l}\text { Irradiated Fuel } \\
\text { Separations }\end{array}$ & $\begin{array}{l}\text { Advanced Fuel } \\
\text { Development }\end{array}$ & Space Power Systems \\
\hline $\begin{array}{l}\text { High-Temperature } \\
\text { Reactor }\end{array}$ & Fast Spectrum Reactor & $\begin{array}{l}\text { Safeguards and } \\
\text { Security }\end{array}$ & $\begin{array}{l}\text { Modeling and } \\
\text { Simulation }\end{array}$ \\
\hline
\end{tabular}

The Fuel and Radiation Laboratory at Texas A\&M University was established to study current issues in the nuclear fuel cycle and reactor component development, including materials and chemical processing, advanced fuels and materials, and waste immobilization. Equipment for fuel development includes high temperature furnaces, two inert atmosphere gloved boxes, and a 90-ton hydraulic press. These may be configured for casting, instrumented sintering, cold or hot pressing, and hot extrusion. The laboratory is equipped and approved for handling, testing, and characterization of radioactive materials.

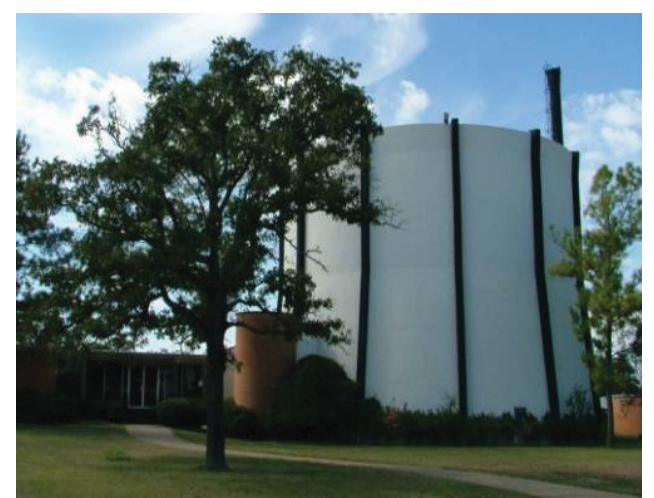

The Nuclear Science Center at Texas A\&M

Equipment for radiation studies includes five accelerators with terminal voltages from $10 \mathrm{keV}$ to $1.7 \mathrm{MeV}$ with energies from a few hundred $\mathrm{eV}$ to a few million $\mathrm{eV}$. The accelerator facilities can be used for radiation simulation of reactor components with high displacements per atom. Displacements per atom up to 100 can be achieved with the sample heated up to $1200 \mathrm{~K}$. Ion irradiation and in situ sample characterization including ion beam analysis, electrical conductivity and heat conductivity measurements can be performed. The high displacements per atom irradiation experiments can provide valuable information for comparison studies using the Advanced Test Reactor at Idaho National Laboratory to gain fundamental understanding of radiation induced materials degradation.

The Nuclear Science Center has served the university's students, university researchers, other academic and non-academic research, and commercial users for nearly 40 years. The Nuclear Science Center facilities include three counting laboratories with five available Germanium detectors, a three-station pneumatics sample transfer system, a delayed neutron counting laboratory, a real-time or film neutron radiography system, a large neutron/gamma irradiation cell, two neutron beam ports and a fast-neutron irradiator. We also perform manufacturing development and design for injectable brachy therapy, sources, and flash polymerization of silicone monomers for preservation of nautical artifacts.

The Nuclear Science Center Reactor achieved first criticality in 1961. The Nuclear Science Center Reactor is a $1.0 \mathrm{MW}$, pool-type TRIGA reactor with low-enriched uranium fuel and pulse capability. Currently, Nuclear Science Center Reactor produces radio isotopes for medical research and for tracer studies. Our tracer production, including Ar-41 and other tracer gases, supports areas as diverse as activation of piston rings for engine wear studies and down borehole tracers for oil exploration and production. The Nuclear Science Center provides Neutron Activation Analysis for a wide range of uses, including micronutrient uptake in cancer cells and forensic analysis. Osmotic films to be used as a filtration media are also produced. 


\section{University of California, Berkeley Nuclear Engineering Laboratories}

Location: University of California, Berkeley

Currently Supporting: Multiple programs

Status: Operational and adding more capability homeland security

Remarks: Unique materials characterization, thermal hydraulics and capabilities

\begin{tabular}{|l|l|l|l|}
\hline Potential Program R\&D Applications are Shaded \\
\hline Light Water Reactors & $\begin{array}{l}\text { Irradiated Fuel } \\
\text { Separations }\end{array}$ & $\begin{array}{l}\text { Advanced Fuel } \\
\text { Development }\end{array}$ & Space Power Systems \\
\hline $\begin{array}{l}\text { High-Temperature } \\
\text { Reactor }\end{array}$ & Fast Spectrum Reactor & $\begin{array}{l}\text { Safeguards and } \\
\text { Security }\end{array}$ & $\begin{array}{l}\text { Modeling and } \\
\text { Simulation }\end{array}$ \\
\hline
\end{tabular}

Advanced Materials Processing and Characterization Laboratory: Facilities to synthesize novel dispersion strengthened structural materials using a ZoZ Attritor mill, and to characterize materials using positron annihilation spectroscopy, atomic force microscopy and thermal desorption spectroscopy, in addition to more traditional mechanical testing and thermal processing.

Advanced Nuclear Engineering Computing Laboratory: A number of Linux-based, parallel computing clusters, including one 72-node and one 160-node cluster provide access to and experience with high performance computing in neutronics, thermal hydraulics, materials and waste management codes and analysis. Data storage and archiving disk arrays range from 2.5 to $30 \mathrm{~TB}$. The Linux clusters and data storage arrays are connected to visualization and compute workstations via a high-speed network.

Domestic Nuclear Threat Security Laboratory: The Department is transitioning from the Rotating Target Neutron Source to a new tandem accelerator, which will arrive in fall 2008 and will provide a versatile source of energetic particles ranging from 3.5 MeV electrons and positrons to $7 \mathrm{MeV}$ hadrons. The new Homeland Security Laboratory contains several other experimental areas, including a small D-T neutron generator with output to $10^{7} 14 \mathrm{MeV}$ neutrons per second, a Machine Vision Radiographic Imaging Laboratory with a movable full-sized cargo container and various detector arrays.

Gamma-ray Imaging Laboratory: A 900 square foot facility for the research and development of novel gamma-ray imaging technologies, with the primary objective of using electron tracks within high spatialresolution silicon radiation sensors to improve the directional sensing of incident gamma-rays.

Computational resources simulate electron track formation, drift, and charge spreading within silicon sensors. A new silicon electron track sensor is currently under development that will image and timeresolve individual electron tracks to within 10 micron and 1 micro-second, respectively. The Laboratory is currently developing a 96 sq. ft. class 10000 clean room with a class 100 clean bench and double-sided, cold probe station, with an HP4284 LCR for new detector diagnostics.

Thermal Hydraulics Laboratory: Three unique facilities provide experimental validation for models of the Pebble Bed Advanced High-Temperature Reactor design. The Pebble Recirculation Experiment uses polyethylene spheres and water with a density ratio matching that of pebbles to salt, as an Integral Effects Test to verify pebble injection, pebble bed movement, and pebble extraction in defueling. The Scaled High Temperature Heat Transfer experiment is used to measure mixed-convection heat transfer coefficients for vertical upward flow in a heated cylindrical channel. Scaled High Temperature Heat Transfer will also verify the steady state and transient heat transfer and flow resistance characteristics of heater modules for future pebble bed mixed convection heat transfer experiments. The Passive Rod Insertion Scaled Model experiment has demonstrated passive insertion of a shutdown rod by buoyancy forces under scaled advanced high-temperature reactor conditions for a loss of heat sink transient without scram. 


\section{University of Cincinnati Nuclear and Radiological Engineering}

Location: University of Cincinnati

Currently Supporting: Health physics; nuclear and radiological safety \& health engineering

Status: Operating

Potential Program R\&D Applications are Shaded

\begin{tabular}{|l|l|l|l|}
\hline Light Water Reactors & $\begin{array}{l}\text { Irradiated Fuel } \\
\text { Separations }\end{array}$ & $\begin{array}{l}\text { Advanced Fuel } \\
\text { Development }\end{array}$ & Space Power Systems \\
\hline $\begin{array}{l}\text { High-Temperature } \\
\text { Reactor }\end{array}$ & Fast Spectrum Reactor & $\begin{array}{l}\text { Safeguards and } \\
\text { Security }\end{array}$ & $\begin{array}{l}\text { Modeling and } \\
\text { Simulation }\end{array}$ \\
\hline
\end{tabular}

Nuclear Radiochemistry: The University of Cincinnati Nuclear Radiochemistry Laboratory and the Laboratories for Environmental Radiological Assessment and Measurement include resources to perform high sensitivity alpha and gamma spectrometry, beta proportional and liquid scintillation counting to analyze any type of radioactive material including uranium, thorium, plutonium, and other special nuclear materials. New radiochemical methods include use of neutron activation analysis to perform high sensitivity measurements of natural and technologically-enhanced radioactivity in a variety of biological and environmental samples. Indirect measurement of internally deposited radioactive material is accomplished by radiochemical analysis of samples collected from persons who may have been exposed to a radioactive substance. Samples typically consist of excreta (urine or feces), hair, tissue, or other biological tissue. Measurements of environmental contamination are also performed using samples of soil, vegetation, air, or air filters to determine the content of natural, industrial, or technologically enhanced radioactive material. The laboratory has eighty alpha spectrometers, seven gamma spectrometers, and ample facilities for radiochemical analysis and measurement of nearly any number and type of samples.

Radiation Detection: Design, fabrication and testing of novel radiation detectors for a wide range of applications including surveillance, personnel, occupational and environmental monitoring. Computer simulations using Monte Carlo analysis are conducted during the initial design phase of detector development. Benchmark measurements to confirm predictions from simulations are performed using a wide variety of hardware including analog and digital nuclear instrument modules, photomultiplier tubes and photodiodes, and a full suite of data acquisition software from National Instruments.

In Vivo Radiation Measurements: Direct, in vivo measurements are performed at the University of Cincinnati In Vivo Measurement Laboratory to evaluate whether a person may have inhaled or ingested radioactive materials arising from occupational or environmental exposure, accidents, or incidents of radiological terrorism. Measurement involves placing detectors on the person, usually over the lungs, head or knees, to detect very small quantities of radiation emitted from the organ or tissue suspected of containing the radioactive substance. The laboratory includes two large shielded rooms with thick steel walls to reduce interferences that arise from natural background radiation. Each room has at least one array of detectors with computerized digital signal processors for performing high sensitivity measurements of internally deposited radioactive materials.

Anthropometric Calibration Standards: New anthropometric calibration standards (phantoms) have been developed for calibrating direct in vivo measurements of uranium and transuranic radionuclides that may be deposited in the lungs, liver, and skeleton. Calibration phantoms have also been developed to measure radioactive materials in wounds. These phantoms are used world-wide at nearly all national and governmental laboratories 


\section{University of Florida Facilities}

Location: University of Florida, Gainesville, Florida

Currently Supporting: Multiple programs

Status: Fully operational

\begin{tabular}{|l|l|l|l|}
\hline Potential Program R\&D Applications are Shaded \\
\hline Light Water Reactors & $\begin{array}{l}\text { Irradiated Fuel } \\
\text { Separations }\end{array}$ & $\begin{array}{l}\text { Advanced Fuel } \\
\text { Development }\end{array}$ & Space Power Systems \\
\hline $\begin{array}{l}\text { High-Temperature } \\
\text { Reactor }\end{array}$ & Fast Spectrum Reactor & $\begin{array}{l}\text { Safeguards and } \\
\text { Security }\end{array}$ & $\begin{array}{l}\text { Modeling and } \\
\text { Simulation }\end{array}$ \\
\hline
\end{tabular}

The University of Florida Training Reactor (was started in 1959 as a $10 \mathrm{~kW}$ reactor and subsequently upgraded to $100 \mathrm{~kW}$ with total flux levels up to $4.5 \mathrm{E} 12 \mathrm{n} / \mathrm{cm}^{2} \mathrm{sec}$. The fuel was converted to low enriched uranium-silicide plate-type fuel in September 2006, and in 2007 a major initiative on design, licensing and construction of a fully digital reactor control system has begun. A major goal is to establish a center for I\&C benchmarking and testing. The University of Florida Training Reactor is used for education, training and research, neutron activation analysis, materials irradiation, neutron absorber evaluations, and radiolysis experimentation for space application. Eleven access ports are available, including six beam ports, three vertical ports accessing the higher flux levels between the fuel boxes, a pneumatic sample insertion/removal capability, and a thermal column. Currently, these access ports are being characterized to equip each port with a unique experimental capability. Work is underway to build a submerged fuel burnup reconstruction facility. The University of Florida Training Reactor is supported by a hot cell and a Neutron Activation Analysis lab for post-irradiation experimentation and processing. For further information, see http://www.nre.ufl.edu/facilities/uftr.php.

The Florida Institute of Nuclear Detection and Security was established in 2004 as a design-basis center for research, development, testing, and engineering projects that directly satisfy critical nuclear detection problems facing both the State of Florida and our nation. Florida Institute of Nuclear Detection and Security has been involved in the design and optimization of active interrogation systems, such as the Westinghouse Pulse Gamma Neutron Activation Analysis, x-ray backscattering by selective detection, and development of unfolding algorithms such as ASEDRA. For further information, see http://finds.nre.ufl.edu.

The University of Florida Training Reactor and the Florida Institute of Nuclear Detection and Security utilize the Progress Energy Florida Advanced Radiation Detection Laboratory, which was established in 2006 for development of advanced radiation detection and measurement technologies, and the Particle Transport \& Distributed Computing, which was established in 2001 to provide PC-clusters and advanced neutronics codes and tools for 3-D simulation of nuclear systems. For further information, see http://ufttg.nre.ufl.edu/hpc/ufttghpc.html

The Innovative Nuclear Space Power and Propulsion Institute at the University of Florida was founded in 1985 for research over a broad range of activities including the testing, model development and validation for High Temperature Gas Cooled Reactors and their associated Reactor Cavity Cooling Systems. This includes feasibility analysis for ultra-compact nuclear power reactor concepts and experimental and theoretical research to establish the fundamental properties of the high temperature materials and processes used in space power reactors. Processing techniques have been developed for producing high quality, single-phase solid-solution mixed carbides and carbonitrides of uranium and zirconium, niobium, and hafnium. For further information, see http://www.inspi.ufl.edu. 


\section{Nuclear Engineering Materials and Separations Laboratory}

Location: University of Idaho: Moscow and Idaho Falls, Idaho

Currently Supporting: "Acquisition of a Simultaneous Thermal Analyzer for Global Nuclear Energy Partnership Research and Training at University of Idaho," DOE, and other multiple Idaho National Laboratory/Center for Advanced Energy Studies programs

Status: Fully operational

\begin{tabular}{|l|l|l|l|}
\hline Potential Program R\&D Applications are Shaded \\
\hline Light Water Reactors & $\begin{array}{l}\text { Irradiated Fuel } \\
\text { Separations }\end{array}$ & $\begin{array}{l}\text { Advanced Fuel } \\
\text { Development }\end{array}$ & Space Power Systems \\
\hline $\begin{array}{l}\text { High-Temperature } \\
\text { Reactor }\end{array}$ & Fast Spectrum Reactor & $\begin{array}{l}\text { Safeguards and } \\
\text { Security }\end{array}$ & $\begin{array}{l}\text { Modeling and } \\
\text { Simulation }\end{array}$ \\
\hline
\end{tabular}

The laboratory specializes in electrochemical methods for separations. It is equipped with several computerized potentiostat/galvanostats, furnaces for work with molten salts, and glove boxes for work under controlled atmosphere. Analytical equipment capabilities include characterization by scanning electron microscopy (Tescan, Vega II) and energy dispersive spectroscopy (IXRF).

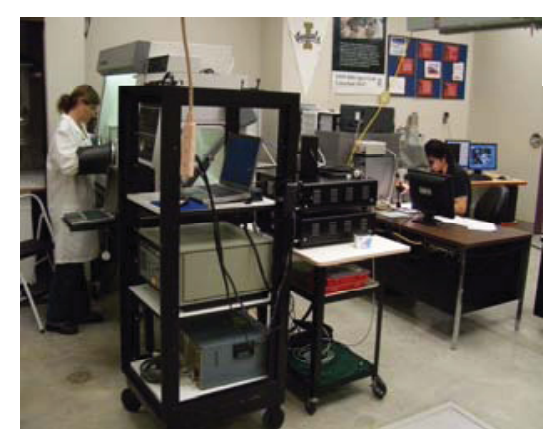

UI NE Laboratory, Moscow, ID

Other specialized items include STA equipment from NETZSCH which can be used to perform very high temperature (up to $1773 \mathrm{~K}$ ) differential scanning calorimetry, differential calorimetry and thermogravimetric analysis experiments on a variety of materials under controlled atmosphere. The heating and cooling rates are programmable and the data acquisition system is fully automated. This system is meant to be used for thermal analysis of advanced cladding and surrogate fuel materials. This facility cannot be used for radioactive materials.

In addition, analysis of gaseous phases is achieved by the fully dedicated gas chromatograph (Stanford Research Instruments), while the liquid phases are analyzed by atomic absorption spectrometry (Varian, Spectra 10/20). Numerous other

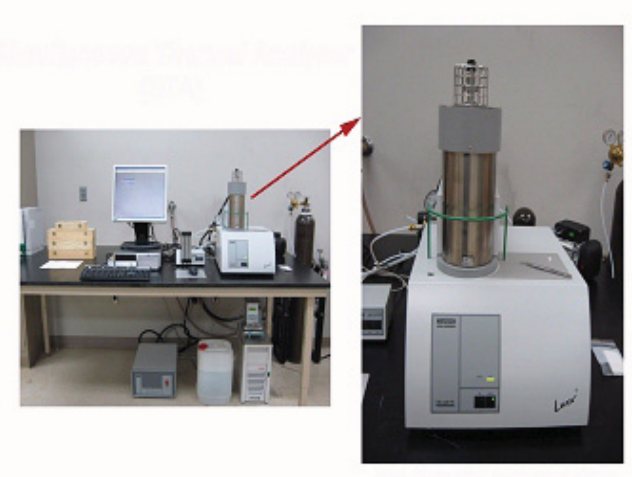
equipment, found in a typical analytical laboratory (scales, microscopes, etc.), is also available.

UI Simultaneous

Thermal Analyzer (left) and an enlarged view of the main unit (right) 


\section{University of Massachusetts Lowell Radiation Laboratory}

Location: University of Massachusetts Lowell

Currently Supporting: Various University of Massachusetts Lowell education and research programs, and industry users

Status: Operational

\begin{tabular}{|l|l|l|l|}
\hline Potential Program R\&D Applications are Shaded \\
Light Water Reactors & $\begin{array}{l}\text { Irradiated Fuel } \\
\text { Separations }\end{array}$ & $\begin{array}{l}\text { Advanced Fuel } \\
\text { Development }\end{array}$ & Space Power Systems \\
\hline $\begin{array}{l}\text { High-Temperature } \\
\text { Reactor }\end{array}$ & Fast Spectrum Reactor & $\begin{array}{l}\text { Safeguards and } \\
\text { Security }\end{array}$ & $\begin{array}{l}\text { Modeling and } \\
\text { Simulation }\end{array}$ \\
\hline
\end{tabular}

In addition to pure and applied nuclear physics research, University of Massachusetts Lowell Radiation Laboratory facilities have been used for: simulating space radiation environments; non-destructive testing and analysis; lifetime and design basis accident gamma dose qualification of nuclear power plant components and materials; computational modeling of nuclear reactor dynamics and radiation transport. Other uses include research and development of radiation resistant electronics and materials, and research and development of radiation induced modifications to materials.
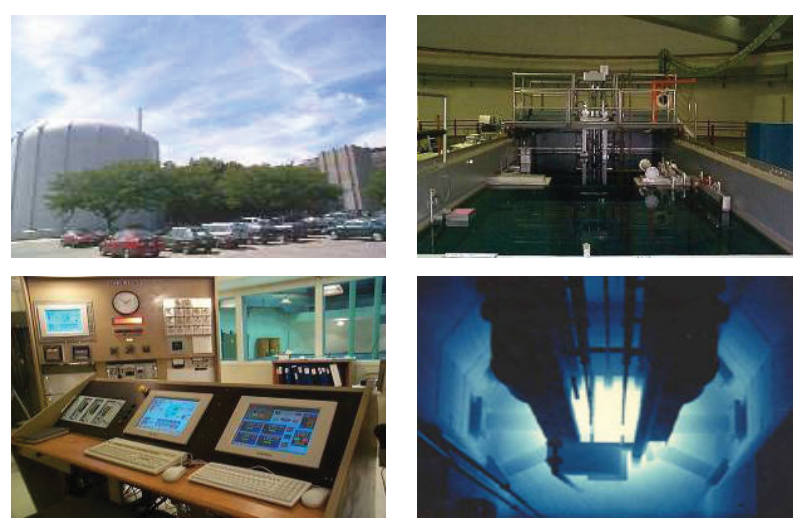

UMLRL facility photos - University of Massachusetts Lowell
The research reactor is a steady-state, swimming pool-type facility. It was originally designed for $5 \mathrm{MW}$ operation and is one of only 3 U.S. university research reactors equipped with a true containment building. It is currently licensed for $1 \mathrm{MW}$, and a license amendment for $2 \mathrm{MW}$ is pending. The University of Massachusetts Lowell Radiation Laboratory is primarily used for neutron activation analysis, digital neutron radiography, and fast neutron irradiation effects on electronics. Multiple in-core thimbles provide a thermal fluence to $2 \mathrm{E} 13 \mathrm{nv}$ and a fast fluence to $1.5 \mathrm{E} 13 \mathrm{nv}$. A large $\left(10,000 \mathrm{~cm}^{3}\right)$ ex-core fast neutron irradiator provides a uniform $1 \mathrm{MeV}$ equivalent fluence of $1 \mathrm{E} 11 \mathrm{nv}$. Three beam ports (6" to 8" diameter), a pneumatic sample transfer system, and thermal column are also available. The reactor control and safety system is digitally instrumented with over 50 web-accessible indicators (including core power level, primary and secondary flow rates, differential pressure measurements, various temperature values, device on/off status indicators, etc.) that can be used for web-based monitoring and limited web-based control.

Several Co-60 gamma irradiation facilities also are located within the reactor containment building. Gamma dose-rates ranging over several orders of magnitude are achievable for various applications. High dose rates, up to $2 \times 10^{4} \mathrm{~Gy} / \mathrm{hr}(2 \mathrm{Mrad} / \mathrm{hr}$ ), are available for materials damage studies (Total Dose Testing). Low dose-rates, down to $36 \mathrm{cGy} / \mathrm{hr}$ (36 rad/hr), are used for enhanced low-dose response sensitivity studies.

While primarily designed for proton production, the accelerator can be configured to produce deuteron, alpha particle or oxygen ion beams in either pulsed or dc mode. The accelerator laboratory includes a number analytical instruments and the capability for fast neutron production and has been used extensively for neutron elastic and inelastic cross section measurements. 


\section{University of Michigan Nuclear Engineering and Radiological Sciences Laboratories}

Location: University of Michigan

Currently Supporting: Multiple programs

Status: Operating

\begin{tabular}{|l|l|l|l|}
\hline Potential Program R\&D Applications are Shaded \\
\hline Light Water Reactors & $\begin{array}{l}\text { Irradiated Fuel } \\
\text { Separations }\end{array}$ & $\begin{array}{l}\text { Advanced Fuel } \\
\text { Development }\end{array}$ & Space Power Systems \\
\hline $\begin{array}{l}\text { High-Temperature } \\
\text { Reactor }\end{array}$ & Fast Spectrum Reactor & $\begin{array}{l}\text { Safeguards and } \\
\text { Security }\end{array}$ & $\begin{array}{l}\text { Modeling and } \\
\text { Simulation }\end{array}$ \\
\hline
\end{tabular}

Irradiated Materials Testing Complex - provides the capability to conduct high temperature corrosion and stress corrosion cracking of neutron irradiated materials and to characterize the fracture surfaces after failure. The laboratory consists of a high temperature autoclave, circulating water loop, load frame and servo motor for conducting constant extension rate tensile and crack growth rate tests in subcritical or supercritical water up to $600^{\circ} \mathrm{C}$.

Neutron Science Laboratory - provides a hands-on neutron measurement experience for students within NERS. The lab is equipped with D-D and D-T neutron generators with a capability of $1 \mathrm{E} 06$ and $\sim 1 \mathrm{E} 10$ neutrons/sec, respectively. The neutron generators are also available for research in NERS and elsewhere within the University who require a neutron radiation field for the conduct of their research. Michigan Ion Beam Laboratory - was established for the purpose of advancing our understanding of ion-solid interactions by providing up-to-date equipment with unique and extensive facilities to support research at the cutting edge of science.

Plasma Science and Technology Laboratory - the focus is on understanding and applying plasma science to real world problems. Plasma science is a highly interdisciplinary field whose primary subject matter is ionized gas. DC, Rf, and microwave plasmas are investigated over a wide pressure window. The lab has four major thrust areas: plasma space propulsion, plasma processing, environmental mitigation, and energy conversion.

Position-Sensing Radiation Detector Laboratory - is dedicated to the development of roomtemperature semiconductor radiation detectors. These instruments are being developed for applications in nuclear non-proliferation, homeland security, astrophysics, planetary sciences, medical imaging, highenergy physics experiments.

High Temperature Corrosion Laboratory - provides the capability to conduct corrosion, stress corrosion cracking, and hydrogen embrittlement tests in high temperature aqueous environments and, in particular, simulated light water reactor environments.

Detection for Nuclear Nonproliferation Laboratory - is used to explore novel techniques for radiation detection and characterization for nuclear nonproliferation and homeland security applications.

Radiation Imaging Laboratory - the goal is to develop high- energy gamma ray imaging systems for industrial, space, homeland security, and medical applications.

Radiation Effects and Nanomaterials Laboratory - is for the preparation and analysis of materials for the study of radiation effects and nanoscience/technology. The laboratory facilities include: a Regarku Miniflex x-ray diffractometer, a high temperature furnace, a Gatan precision ion polishing workstation, an ultramicrotomy workstation, a carbon coater, and other standard equipment for TEM sample preparation.

Radiological Health Engineering Laboratory - includes equipment and space for the development and testing of new instruments and systems for application to specific radiological health problems. Work is concentrated on practical systems and radiation measurements methods deployable within the immediate future. 


\section{University of Missouri Research Reactor}

Location: University of Missouri-Columbia

Currently Supporting: Multiple programs

Status: Fully operational

Remarks: Life-extension upgrades completed and re-license application submitted to the Nuclear Regulatory Commission

\begin{tabular}{|l|l|l|l|}
\hline Potential Program R\&D Applications are Shaded \\
\hline Light Water Reactors & $\begin{array}{l}\text { Irradiated Fuel } \\
\text { Separations }\end{array}$ & $\begin{array}{l}\text { Advanced Fuel } \\
\text { Development }\end{array}$ & Space Power Systems \\
\hline $\begin{array}{l}\text { High-Temperature } \\
\text { Reactor }\end{array}$ & Fast Spectrum Reactor & $\begin{array}{l}\text { Safeguards and } \\
\text { Security }\end{array}$ & $\begin{array}{l}\text { Modeling and } \\
\text { Simulation }\end{array}$ \\
\hline
\end{tabular}

At $10 \mathrm{MW}$, MURR is the highest powered university research reactor in the U.S. Since 1977, MURR has operated at a schedule of more than 150 hours/week, every week of the year. During this period, the reactor has been on line for approximately $90 \%$ of all available time. The pressurized reactor uses highly enriched uranium, is light-water moderated and cooled, and is beryllium reflected. Eight fuel elements in the fuel zone form the core. Each fuel element is assembled from 24 fuel plates; each plate is a sandwich of uranium aluminide fuel with aluminum cladding, held in place by side plates and end boxes. The active core is $30 \mathrm{~cm}$ in diameter and $61 \mathrm{~cm}$ tall, with an active core volume of 33 liters.

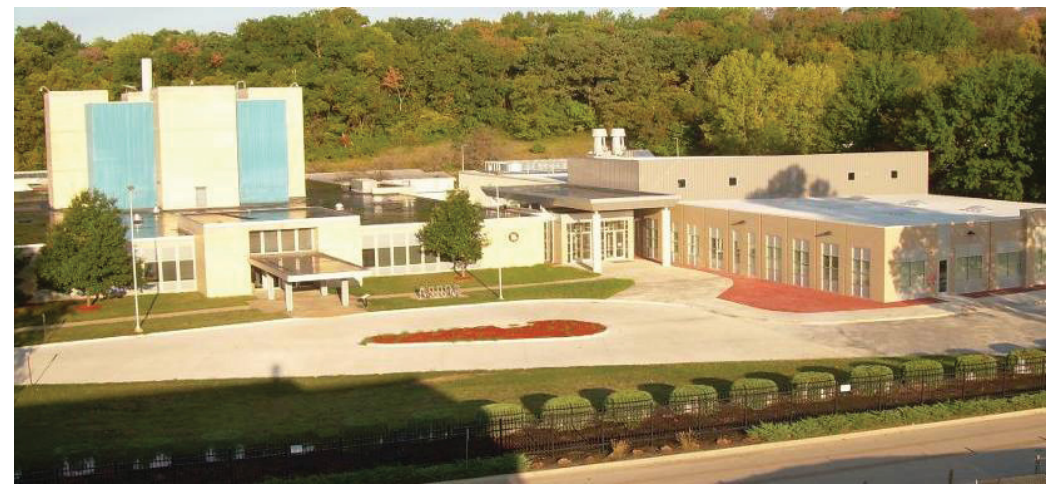

University of Missouri Research Reactor Center
Samples can be irradiated in the flux trap, the graphite reflector region, beam tubes, and in the bulk pool. The flux trap has a 4.5 " annulus and provides a peak flux of $6 \times 10^{14}$ $\mathrm{n} / \mathrm{cm}^{2} \mathrm{sec}$. The graphite reflector region provides a much larger volume for irradiations and has the added advantage that even large targets can be accessed when the reactor is at full power. The irradiation positions are approximately 30 " tall and have diameters ranging from 1 " to 6 " and

peak fluxes ranging from 8.0 to $1.0 \times 10^{13} \mathrm{n} / \mathrm{cm}^{2} \mathrm{sec}$. The facility also has 2 pneumatically controlled irradiation positions with peak fluxes of 8.0 and $6.0 \times 10^{13} \mathrm{n} / \mathrm{cm}^{2} \mathrm{sec}$. The bulk pool facilities allow for larger or specially shielded irradiation positions. Currently there are three 5" O.D. positions 30" in length. The peak flux for the bulk pool facility is approximately $6 \times 10^{12} \mathrm{n} / \mathrm{cm}^{2} \mathrm{sec}$. Six neutron beam tubes surround the Be reflector and have source fluxes of $1.2 \times 10^{14} \mathrm{n} / \mathrm{cm}^{2} \mathrm{sec}$. A state-of-the art triple-axis spectrometer occupies one beam tube and a high-resolution powder diffractometer with a resolution of $1.5 \times 10^{-3}(\Delta \mathrm{d} / \mathrm{d})$ is located on a second beam tube. A third beam tube houses a small-animal irradiation facility dedicated to boron neutron capture research.

MURR's operating schedule and our new external beam 16.7 MV cyclotron make the facility a unique national resource for human use diagnostic and therapeutic isotope production. In addition, the facility actively supports research programs in radiation effects on materials and neutron scattering studies of hard and soft matter. 


\section{University of Nevada, Las Vegas Radiochemistry Research Laboratories}

Location: University of Nevada, Las Vegas

Currently Supporting: Multiple programs

Status: The radiochemistry research laboratories are $100 \%$ operational.

Remarks: The radiochemistry program at University of Nevada, Las Vegas is housed primarily in the Harry Reid Center for Environmental Studies

\begin{tabular}{|l|l|l|l|}
\hline Potential Program R\&D Applications are Shaded \\
\hline Light Water Reactors & $\begin{array}{l}\text { Irradiated Fuel } \\
\text { Separations }\end{array}$ & $\begin{array}{l}\text { Advanced Fuel } \\
\text { Development }\end{array}$ & Space Power Systems \\
\hline $\begin{array}{l}\text { High-Temperature } \\
\text { Reactor }\end{array}$ & Fast Spectrum Reactor & $\begin{array}{l}\text { Safeguards and } \\
\text { Security }\end{array}$ & $\begin{array}{l}\text { Modeling and } \\
\text { Simulation }\end{array}$ \\
\hline
\end{tabular}

The Harry Reid Center for Environmental Studies provides facilities with multiple analysis capabilities in a friendly, secure environment working with others who have years of experience working with radioactive material. All open areas of the laboratories are maintained free of radioactive contamination and protective clothing requirements in the labs are kept to a minimum. The primary group focus is work with the actinide series but other radionuclides may be a part of the work as required.

The radiochemistry laboratories at University of Nevada, Las Vegas provide significant analysis capability in an atmosphere that provides for safety, security, and radiological controls. Some of the analysis capabilities are: inductively coupled plasma mass spectrometry (3), inductively coupled plasma atomic emission spectroscopy, gamma spectroscopy, liquid scintillation counting and spectroscopy, alpha spectroscopy, liquid chromatography, ion chromatography, UV-Visible-IR spectroscopy, FT-IR spectroscopy, transmission electron microscopy, surface area analysis, $x$-ray diffraction (single crystal and powder), neutron multiplicity detectors (3He tubes), and other counting systems for alpha, beta, and gamma emissions, and electron microscopy and imaging.

In order to support these analysis capabilities, the center provides for sample preparation capabilities as follows: freeze drying, high temperature tube furnaces, an arc furnace with remote electrodes, microtome, minitome, sample polishers, filtered ventilation hoods and unfiltered ventilation hoods, glove boxes, centrifuges, ball mills, balances, electroplaters, etc. The labs also provide for the safety of personnel with first aid kits, fire extinguishers, portable radiation detectors, dosimetry, emergency showers, emergency eye-wash stations, lab coats, etc.

Programs currently being supported include Advanced Fuel Cycle R\&D, Global Nuclear Energy Partnership Readiness, Deep Burn Reactor Program, Laser Inertialconfinement Fusion/fission Energy Project, Fundamental Surface Reactions Involved in the Sorption and Desorption of Radionuclides Project, Uranium

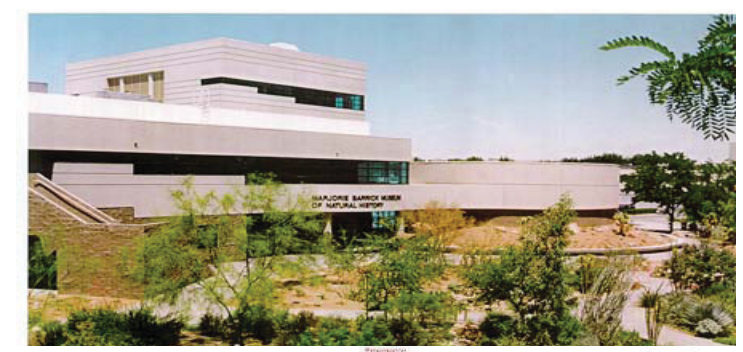
Visualization Chemistry Project, DARPA Radiation Decontamination Project, and the Nevada Risk Assessment/Management Program. 


\section{University of New Mexico Nuclear Facilities}

Location: University of New Mexico, Albuquerque, NM

Currently Supporting: Multiple Programs

Status: Fully Operational

Remarks: Reactor and most lab equipment for nonproliferation work currently available. Facility would be enhanced with a cluster computing system and additional multichannel detection equipment.

Potential Program R\&D Applications are Shaded

\begin{tabular}{|l|l|l|l|}
\hline Light Water Reactors & $\begin{array}{l}\text { Irradiated Fuel } \\
\text { Separations }\end{array}$ & $\begin{array}{l}\text { Advanced Fuel } \\
\text { Development }\end{array}$ & Space Power Systems \\
\hline $\begin{array}{l}\text { High-Temperature } \\
\text { Reactor }\end{array}$ & Fast Spectrum Reactor & $\begin{array}{l}\text { Safeguards and } \\
\text { Security }\end{array}$ & $\begin{array}{l}\text { Modeling and } \\
\text { Simulation }\end{array}$ \\
\hline
\end{tabular}

The University of New Mexico has a small operating nuclear reactor on campus capable of supporting research in cross sections and small sample reactivity measurements. Some work has been done recently developing experiments in support of actinide separations and nuclear criticality safety for irradiated fuel separations. The University of New Mexico Center of Excellence in nuclear nonproliferation science and technology supports the planning, coordination and conduct of fundamental research in nonproliferation science and technology relevant to advanced fuel cycles. Specific focus of current work

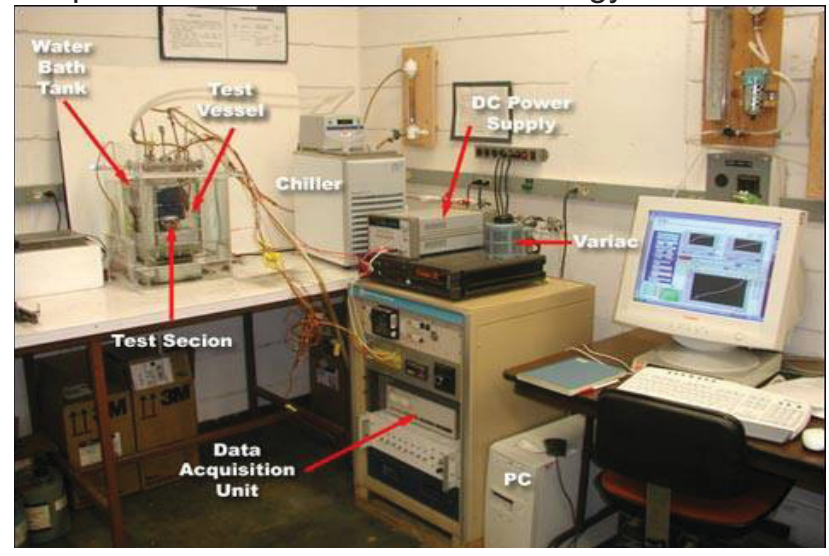

The Experimental Pool Boiling Facility at the Institute for Space and Nuclear Power Studies includes: Analyzing new and next generation radiation detectors and detection systems identifying opportunities to increase their effectiveness using transport computer codes, developing novel approaches to neutron and photon instrumentation for the purpose of detecting and measuring nuclear materials relevant to nonproliferation and nuclear security, and developing new, comprehensive deterministic and stochastic neutral/charged particle transport capability encompassing mathematical modeling and analysis, with specific application to measurement and detection of nuclear materials. University of New Mexico also has an international reputation in the area of training engineers, operators, and managers in nuclear criticality safety and in the use of major criticality codes

(TWODANT, MCNP, and KENO) and in validation approaches.

The Institute for Space and Nuclear Power Studies possesses a wide range of technical expertise, including: design, thermal-hydraulics and neutronics analysis of gas-cooled, liquid metal-cooled and heat pipe-cooled space nuclear reactors; design optimization and performance of heat pipe radiators; thermal management of Space Nuclear Reactor Power Systems; transient modeling of heat pipes, including the startup from a frozen state; transient operation, safety and autonomous control of fully-integrated Space Nuclear Reactor Power Systems; modeling, design optimization and vacuum testing of high-temperature energy conversion devices, such as thermionic diodes, segmented and non-segmented thermoelectric devices, and Alkali-Metal Thermal-to-Electric Converters; and design, optimization, and thermal and stress analyses of segmented and cascaded thermoelectric converters for Space Nuclear Reactor Power Systems and Advanced Radioisotope Power Systems. 


\section{University of South Carolina Research Center of Excellence in Nuclear Science and Engineering}

Location: University of South Carolina

Currently Supporting: Advanced Fuel Cycle Initiative, Generation IV, National Hydrogen Initiative

Status: Fully operational

Remarks: Lab and program development continuing

\begin{tabular}{|l|l|l|l|}
\hline Potential Program R\&D Applications are Shaded \\
\hline Light Water Reactors & $\begin{array}{l}\text { Irradiated Fuel } \\
\text { Separations }\end{array}$ & $\begin{array}{l}\text { Advanced Fuel } \\
\text { Development }\end{array}$ & Space Power Systems \\
\hline $\begin{array}{l}\text { High-Temperature } \\
\text { Reactor }\end{array}$ & Fast Spectrum Reactor & $\begin{array}{l}\text { Safeguards and } \\
\text { Security }\end{array}$ & $\begin{array}{l}\text { Modeling and } \\
\text { Simulation }\end{array}$ \\
\hline
\end{tabular}

The University of South Carolina has a number of laboratories performing research related to the shaded areas above and additional laboratories are planned as part of a Research Center of Excellence in Nuclear Science and Engineering recently awarded by the State of South Carolina. Chief among these facilities is the Nuclear Materials Laboratory.

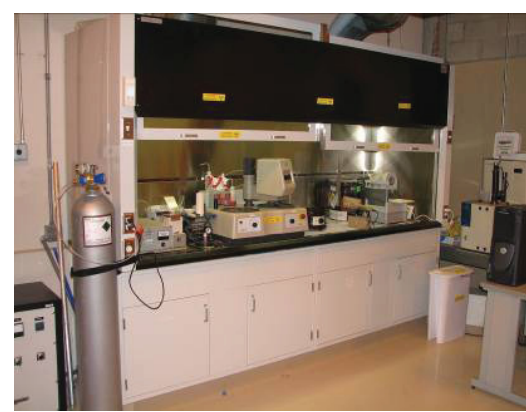

Sample preparation equipment for fuel microscopy and microanalysis

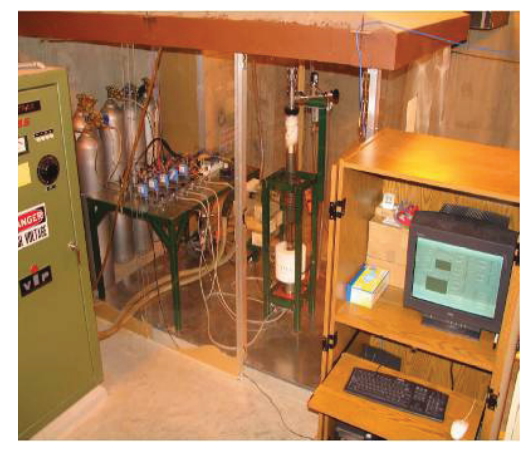

Fluidized bed, chemical vapor deposition system for advanced coated particle fuel development
The Nuclear Materials Laboratory is equipped and licensed for working with uranium and thorium based fuels within radiological hoods and inert atmosphere gloveboxes for pyrophoric materials. Metallographic and sample preparation tools are used for preparing materials for analysis in University of South Carolina microscopy and microanalysis instruments or for delivery to partner institutions. High temperature, controlled atmosphere furnaces are used for advanced fuel fabrication and testing. Induction heated furnaces are used to $3000 \mathrm{~K}$ and a longer duration tube furnace is used to temperatures up to $1900 \mathrm{~K}$. A fluidized-bed, chemical vapor deposition system is used for coating of fuel particles including advanced TRISO fuels. Other instruments used for nuclear fuels characterization include particle size, porosimetry, density, and surface area analysis.

Thermogravimetric and differential scanning calorimetry instruments are also employed in these studies at temperatures up to $2250 \mathrm{~K}$.

High performance computing facilities are used to analyze and model nuclear systems, components, and fuels. Modeling and simulation codes and tools are employed for neutronic, thermal hydraulic, computational fluid dynamics, thermochemical, safety and risk, shielding, and finite element analyses. Sample code packages include MCNP, SCALE, FACT, ABAQUS, Comsol Multiphysics, etc.

Thermal hydraulic test loops and laboratories are dedicated to studies of enhanced heat transfer, fluid flow, pressure drop and other phenomena associated with nuclear fuel rods and assemblies. Stereo vision (3D) micro Particle Image Velocimetry and micro-Planar Laser Induced Fluorescence techniques are used to examine the impact of nanofluids on the development and performance of thermal and hydrodynamic boundary layers and to allow for a visual investigation of the flow field and the impact of shear-thinning. 


\section{Nuclear Research Facilities at the University of Tennessee}

Location: Knoxville, TN

Currently Supporting: Multiple nuclear and radiological engineering programs

Status: Facilities are fully operational

\begin{tabular}{|l|l|l|l|}
\hline Potential Program R\&D Applications are Shaded \\
\hline Light Water Reactors & $\begin{array}{l}\text { Irradiated Fuel } \\
\text { Separations }\end{array}$ & $\begin{array}{l}\text { Advanced Fuel } \\
\text { Development }\end{array}$ & Space Power Systems \\
\hline $\begin{array}{l}\text { High-Temperature } \\
\text { Reactor }\end{array}$ & Fast Spectrum Reactor & $\begin{array}{l}\text { Safeguards and } \\
\text { Security }\end{array}$ & $\begin{array}{l}\text { Modeling and } \\
\text { Simulation }\end{array}$ \\
\hline
\end{tabular}

University of Tennessee Nuclear Engineering Department facilities available on campus include two radiation measurement laboratories, a radiochemical laboratory, two natural uranium subcritical facilities (one graphite moderated and one water moderated), a nuclear materials welding chamber, two accelerator target design test facilities, a flow induced vibration test facility for main steam lines, a 1/8 Scale Flow Test model of the AP-600, numerous reactor plant instrumentation and control testing equipment, the Maintenance and Reliability Center, the Scintillation Materials Research Center, and a low-level waste storage facility. Equally important, University of Tennessee Nuclear Engineering students and faculty utilize many of the one-of-a-kind facilities at the nearby (30 miles) Oak Ridge DOE complex for both teaching and research (e.g., the 85 MW High Flux Isotope Reactor). University of Tennessee Nuclear Engineering also has access to linear accelerators at the nearby (1 mile) Thompson Cancer Center and the medical imaging facilities at the University of Tennessee Memorial Research Hospital, which is located on campus.

Radiation measurement laboratories are equipped for characterization of photon and neutron sensitive detection equipment. The labs consist of six workstations each of which has its own PC and workspace.

Numerous Reactor Plant Instrumentation and Control Equipment, e.g., a two-tank water loop built to study control strategies using Proportional-Integral-Derivative Control and Model Predictive Control, and to study autonomous control.

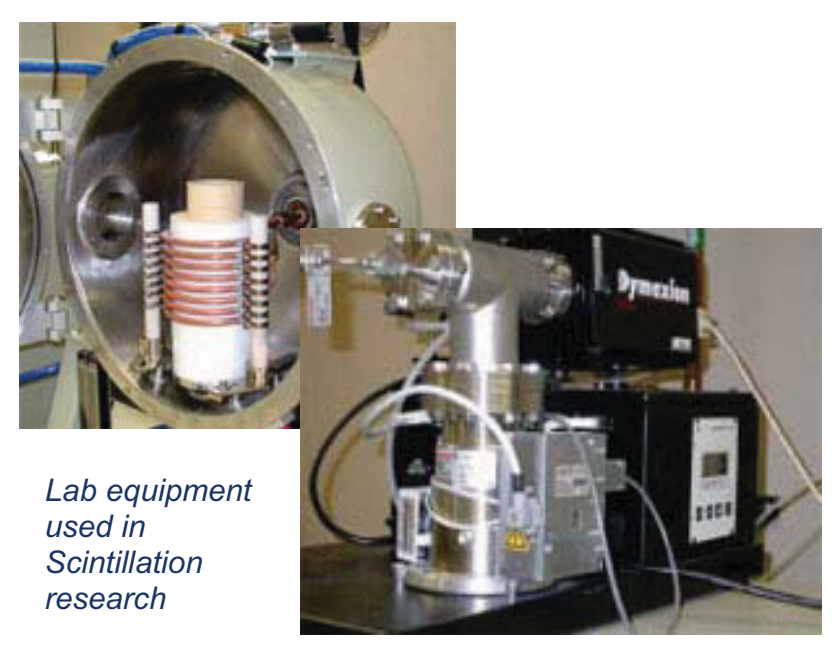

The Scintillation Materials Research Center has capabilities and expertise in scintillation materials synthesis and material characterization. The synthesis lab is equipped for single crystal growth under controlled conditions. The characterization lab is equipped to measure scintillation, optical, and physical properties of scintillators.

University Computing Facilities include a Unix system and a Linux cluster. University of Tennessee Nuclear Engineering has its own clusters for computing and has access to high performance computing facilities. In addition, University of Tennessee Nuclear Engineering has its own undergraduate computing laboratory and all researchers are provided with PCs, which number approximately 100 . 


\section{Nuclear Engineering Teaching Laboratory}

Location: The University of Texas at Austin (Austin, TX)

Currently Supporting: Citizens of Texas, the United States., and the international community; current programs with Department of Defense, DOE, Nuclear Regulatory Commission, and others

Status: Fully operational on a standard 40-hour per week basis

\begin{tabular}{|l|l|l|l|}
\hline Potential Program R\&D Applications are Shaded \\
\hline Light Water Reactors & $\begin{array}{l}\text { Irradiated Fuel } \\
\text { Separations }\end{array}$ & $\begin{array}{l}\text { Advanced Fuel } \\
\text { Development }\end{array}$ & Space Power Systems \\
\hline $\begin{array}{l}\text { High-Temperature } \\
\text { Reactor }\end{array}$ & Fast Spectrum Reactor & $\begin{array}{l}\text { Safeguards and } \\
\text { Security }\end{array}$ & $\begin{array}{l}\text { Modeling and } \\
\text { Simulation }\end{array}$ \\
\hline
\end{tabular}

The University of Texas at Austin began construction on the Nuclear Engineering Teaching Laboratory Mark II TRIGA reactor on January 10, 1986. The reactor went critical on March 12, 1992. The TRIGA reactor has a steady state operating power of $1.0 \mathrm{MW}$ and can pulse up to $1,600 \mathrm{MW}$.

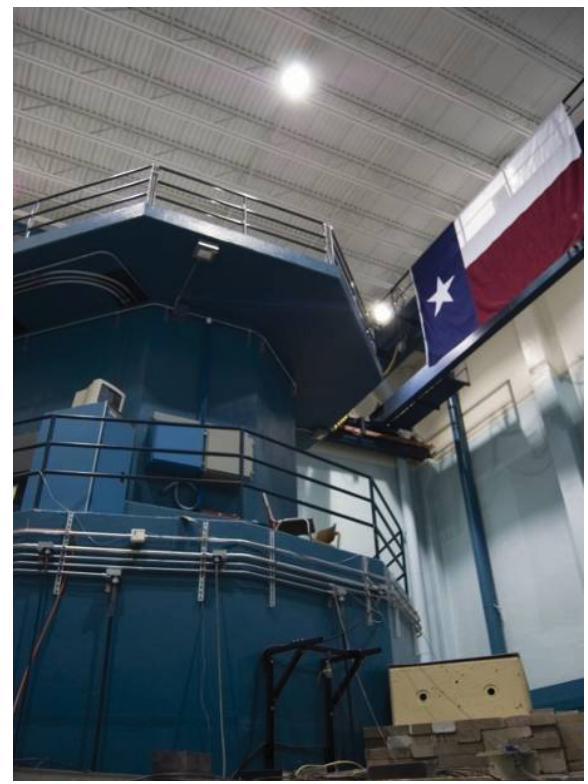

Nuclear Engineering Teaching

Laboratorv reactor bav area
The reactor has multiple in-core irradiation facilities including a $7 \mathrm{~L}(5.7 \mathrm{~cm}$ radius $x 38 \mathrm{~cm}$ height) irradiator, a $3 \mathrm{~L}(3.8 \mathrm{~cm}$ radius $\mathrm{x}$ $38 \mathrm{~cm}$ height) irradiator with optional cadmium lining, a central thimble irradiator, a rotary specimen rack, and a pneumatic injection rack irradiator. The maximum thermal neutron flux in the core is $1 \times 10^{13} \mathrm{n} \mathrm{cm}^{-2} \mathrm{~s}^{-1}$ and samples may be irradiated at this flux in the central thimble. The rotary specimen rack has a thermal neutron flux of $2 \times 10^{12} \mathrm{n} \mathrm{cm}^{-2} \mathrm{~s}^{-1}$. The epithermal neutron flux in the cadmium lined $3 \mathrm{~L}$ facility is $5 \times 10^{11} \mathrm{n} \mathrm{cm}^{-2} \mathrm{~s}^{-1}$.

The reactor has five beam port facilities. Beam port 1 is currently being developed for a positron source. Beam port 2 has a neutron depth profiling facility. Beam port 3 has a cold neutron source, neutron guides, and a prompt gamma activation analysis facility. Beam port 4 is a radial beam port that is not currently being utilized. Beam port 5 houses a neutron radiography/tomography facility.

The Nuclear Engineering Teaching Laboratory also has numerous alpha, beta, and gamma -ray spectroscopy systems. It has two Compton suppression systems to enhance detection limits for gamma ray spectroscopy and has two beta gamma coincidence systems. There are also alpha spectroscopy and liquid scintillation systems. Laboratory space includes a neutron generator room, a radiochemistry laboratory, counting room, and a sample preparation room

Nuclear Engineering Teaching Laboratory is equipped with a conference room with a Tandberg system for distance learning. There are also computational facilities with parallel computing capabilities. 


\section{Wisconsin Institute for Nuclear Systems}

Location: University of Wisconsin

Currently Supporting: Multiple programs

Status: Fully operational

\begin{tabular}{|l|l|l|l|}
\hline Potential Program R\&D Applications are Shaded \\
\hline Light Water Reactors & $\begin{array}{l}\text { Irradiated Fuel } \\
\text { Separations }\end{array}$ & $\begin{array}{l}\text { Advanced Fuel } \\
\text { Development }\end{array}$ & Space Power Systems \\
\hline $\begin{array}{l}\text { High-Temperature } \\
\text { Reactor }\end{array}$ & Fast Spectrum Reactor & $\begin{array}{l}\text { Safeguards and } \\
\text { Security }\end{array}$ & $\begin{array}{l}\text { Modeling and } \\
\text { Simulation }\end{array}$ \\
\hline
\end{tabular}

The thermal hydraulics group operates a number of flow loops with liquid metals (sodium), liquid salts, high temperature high-pressure water and supercritical carbon dioxide. Experiments are conducted on heat transfer, fluid flow and shock physics both single phase fluids and multiphase fluids. The facilities include: the Tantalus facility consisting of $8,000 \mathrm{ft}^{2}$ of underground laboratory space equipped with $1 \mathrm{MW}$ of electrical power its own 2,000 scfm ventilation system with remote monitoring capabilities and liquid metal fire protection systems; a 25 -ft tall $2,000 \mathrm{ft}^{2}$ high-bay area with a 5 -ton crane system; a 40 -ft tall high-bay area with 5-ton crane and a 30-ft shocktube, with a square internal cross section of $10 \times 10$ in.; a full fabrication shop and several auxiliary laboratories. The thermal hydraulics group also has significant diagnostic equipment.

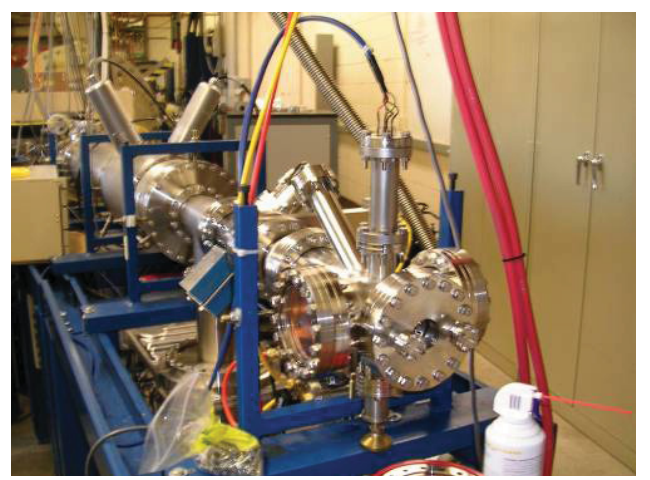

Pelletron Ion Accelerator

The 1.7 MV Tandem accelerator is located in the Engineering Research Building at the University of Wisconsin. A high temperature radiation stage that is specially designed and connected to the beam line of a 1.7 MV Tandem accelerator for irradiating samples up to temperatures of $850^{\circ} \mathrm{C}$ with high-energy protons or other heavy ions while monitoring and controlling the temperature of the samples. Surface analysis using Rutherford Backscattering, Forward Recoil Scattering and Nuclear Reaction Analysis is available.

The University of Wisconsin Extreme Environments Laboratory houses corrosion facilities for exposure to supercritical water, supercritical carbon dioxide, molten salts, and molten lead. An arc melter, rolling mill, and high-temperature furnaces are all available in the Extreme Environments Laboratory and

associated materials characterization facilities.

The University of Wisconsin Nuclear Reactor is a $1 \mathrm{MW}$ open pool reactor fueled with TRIGA fuel. The reactor has several experimental facilities including: One, 40-inch square graphite packed thermal column, four, 6-inch diameter beam ports and one pneumatic tube.

The Computational Materials Group at the University of Wisconsin uses atomic scale modeling to understand and design new materials. Highly accurate ab initio (first-principles) techniques are used to study electronic structure and energetics of smaller systems, and interatomic potential modeling on massively parallel computers to study up to hundreds of millions of atoms. These core approaches are combined with a wide-range of other atomistic methods, including Monte Carlo, coarse graining, data mining, thermodynamics, and statistical physics. 


\section{Summary of Reviewer Comments}

1. Facilities at Clemson, Santa Barbara, and Notre Dame should be included.

2. Application of the requirements of 10 CFR 50, Appendix B, will be necessary if university assets are to be used effectively by DOE in support of a nuclear program. 


\subsection{Major International Facility Descriptions}

\subsubsection{France}

\section{Atalante}

Location: Marcoule, France

Currently Supporting: Multiple Programs

Status: Fully operational

Potential Program R\&D Applications are Shaded

\begin{tabular}{|l|l|l|l|}
\hline Light Water Reactors & $\begin{array}{l}\text { Irradiated Fuel } \\
\text { Separations }\end{array}$ & $\begin{array}{l}\text { Advanced Fuel } \\
\text { Development }\end{array}$ & Space Power Systems \\
\hline $\begin{array}{l}\text { High-Temperature } \\
\text { Reactor }\end{array}$ & Fast Spectrum Reactor & $\begin{array}{l}\text { Safeguards and } \\
\text { Security }\end{array}$ & $\begin{array}{l}\text { Modeling and } \\
\text { Simulation }\end{array}$ \\
\hline
\end{tabular}

The Atalante (Alpha Workshop and Laboratories for the Analysis of Transactinides and Studies on Reprocessing) site is a set of large laboratories and hot cells. The Atalante Project started in the 1980s with one objective- to have, on the same site, all the necessary expertise for CEA to conduct radiochemistry research with highly radioactive materials. It was constructed in two stages; the first stage was completed in 1990 and the second in 1999.

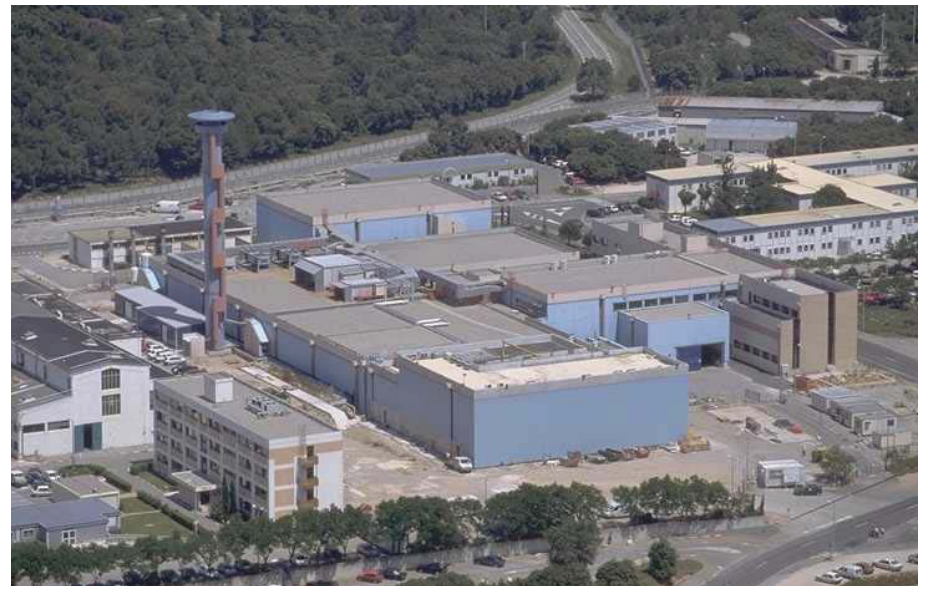

ATALANTE at Marcoule, France
At Atalante, all the reprocessing steps from dissolution of the spent fuel to vitrification can be implemented at pilot plant scale. Research is ongoing for 1 ) development of complementary or new concepts for reprocessing LWR fuels that reduce the cost and minimize the waste; and 2) improving management of high-level waste. This latter objective involves finding and establishing technical and economic feasibility of new methods for extracting long half-life elements (minor actinides and fission products); fabrication and reprocessing transmutation fuels; and improving the performance of waste products necessary for final disposition.

There are five buildings in the hot zone (active material), a general cold building (no nuclear material), and an administrative building. The hot buildings are linked together by a gallery and are built on three levels. There are seven halls containing hot cells with a total of 59 workstations. Seventeen laboratories are equipped with gloveboxes. 


\section{Phenix}

Location: Marcoule, CEA, France

Currently Supporting: Transmutation research

Status: Operating, shutdown scheduled for 2009

Remarks: Shutdown in 2009 is planned

\begin{tabular}{|l|l|l|l|}
\hline Potential Program R\&D Applications are Shaded \\
\hline Light Water Reactors & $\begin{array}{l}\text { Irradiated Fuel } \\
\text { Separations }\end{array}$ & $\begin{array}{l}\text { Advanced Fuel } \\
\text { Development }\end{array}$ & Space Power Systems \\
\hline $\begin{array}{l}\text { High-Temperature } \\
\text { Reactor }\end{array}$ & Fast Spectrum Reactor & $\begin{array}{l}\text { Safeguards and } \\
\text { Security }\end{array}$ & $\begin{array}{l}\text { Modeling and } \\
\text { Simulation }\end{array}$ \\
\hline
\end{tabular}

Phenix is a sodium-cooled fast spectrum test reactor used for testing fuels and materials in a prototypic fast spectrum. Construction began in 1968 and first operation was in 1973. Four upgrades were completed between 1994 and 2003. The core contains 100 subassemblies in a volume of about 9 cubic ft. Since 2003, the power rating has been $350 \mathrm{MWth}$ and $145 \mathrm{MWe}$ (about two-thirds of its initial power rating). The mixed oxide driver fuel provides fast flux of 4.4E15 neutrons per square centimeter per second.

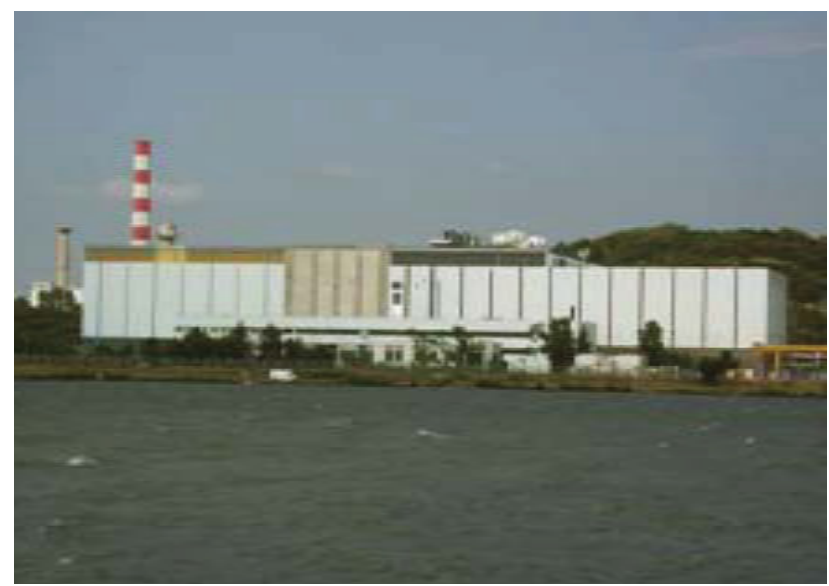

The Phenix fast reactor in Marcoule, France
Over 200 experiments have been conducted since it began operation, while producing well over 24 billion kW of electrical power. Many of the experiments have been for international "customers." The primary current mission is to provide a fast neutron flux for research on transmutation fuels. Shutdown is planned for 2009 on completion of the $56^{\text {th }}$ cycle and 127,000 hours of operation.

The facility includes a hot cell complex for experiment disassembly and non-destructive examination. The cells also have the capability to reconstitute the experiments for reinsertion into the core following examination. 


\subsubsection{Great Britain}

\section{British Technology Centre}

Location: National Nuclear Laboratory, Sellafield Site; Great Britain

Currently Supporting: Multiple Programs

Status: Operational

\begin{tabular}{|l|l|l|l|}
\hline Potential Program R\&D Applications are Shaded \\
\hline Light Water Reactors & $\begin{array}{l}\text { Irradiated Fuel } \\
\text { Separations }\end{array}$ & $\begin{array}{l}\text { Advanced Fuel } \\
\text { Development }\end{array}$ & Space Power Systems \\
\hline $\begin{array}{l}\text { High-Temperature } \\
\text { Reactor }\end{array}$ & Fast Spectrum Reactor & $\begin{array}{l}\text { Safeguards and } \\
\text { Security }\end{array}$ & $\begin{array}{l}\text { Modeling and } \\
\text { Simulation }\end{array}$ \\
\hline
\end{tabular}

The British Technology Centre, operated by NEXIA Solutions, supports primary research programs covering reactor operation and design, fuel processing, decommissioning, and clean-up. It is designed for a wide range of nuclear experimental work with radioactive and non-radioactive materials.

It consists of "cold" and "hot" laboratories for physics, chemistry, and materials science work. Two laboratories have been designated as medium Inventory for plutonium studies, sludge, and cementation trials. Two laboratories are used to develop analytical techniques. Three laboratories are designated as low inventory for waste treatment and characterization. An aerial effluent treatment laboratory supports experimentation to evaluate different systems for removing/ reducing, sampling, and monitoring emissions. An optics and magnetic laboratory is used to support development of process equipment or measurement systems based on lasers and magnets using low or medium levels of radioactive samples and sources.

The facility also provides a line of five remotely operated shielded alpha/beta/gamma/neutron cells. Cells one and two can be linked via an access port to enable larger experiments. Alpha radiation laboratories

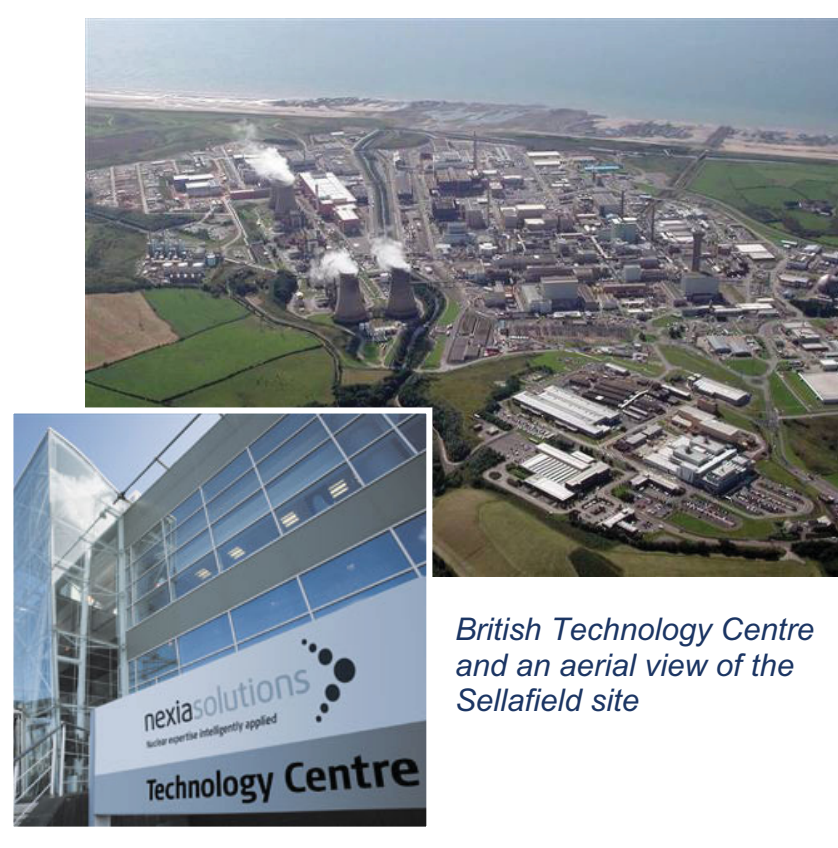
operate essentially as small-scale pilot plants enabling research and development to be carried out on mixed oxide fuels and mixed matrices fuel. They include a product development, rod production, and ceramographical laboratories.

In addition, the facility includes an area capable of accommodating large process and experimental test rigs for testing with low-level radioactive materials. The active rig hall includes a series of six test rig bays in the main area and two in the tower area. The layout of each bay is identical and will accommodate a test rig assembled in a framework 4-m long by 3-m wide and $2.5-\mathrm{m}$ high. If required, the six main bays can accommodate double height rigs. For larger rigs, more than one frame can be locked together. The tower area is $18-\mathrm{m}$ long by $9-\mathrm{m}$ wide. 


\subsubsection{Japan}

\section{Jōyō}

Location: Ōarai, Ibaraki, Japan

Currently Supporting: Fast reactor fuels and materials testing

Status: Operating

\begin{tabular}{|l|l|l|l|}
\hline Potential Program R\&D Applications are Shaded \\
\hline Light Water Reactors & $\begin{array}{l}\text { Irradiated Fuel } \\
\text { Separations }\end{array}$ & $\begin{array}{l}\text { Advanced Fuel } \\
\text { Development }\end{array}$ & Space Power Systems \\
\hline $\begin{array}{l}\text { High-Temperature } \\
\text { Reactor }\end{array}$ & Fast Spectrum Reactor & $\begin{array}{l}\text { Safeguards and } \\
\text { Security }\end{array}$ & $\begin{array}{l}\text { Modeling and } \\
\text { Simulation }\end{array}$ \\
\hline
\end{tabular}

Jōyō is a sodium-cooled fast spectrum test reactor used for testing fuels and materials in a prototypic fast spectrum. It began operation in 1977 in Ōarai, Ibaraki, Japan. It was the first fast test reactor in Japan and plays a key role in establishing the technological basis for fast reactors worldwide. It is operated by

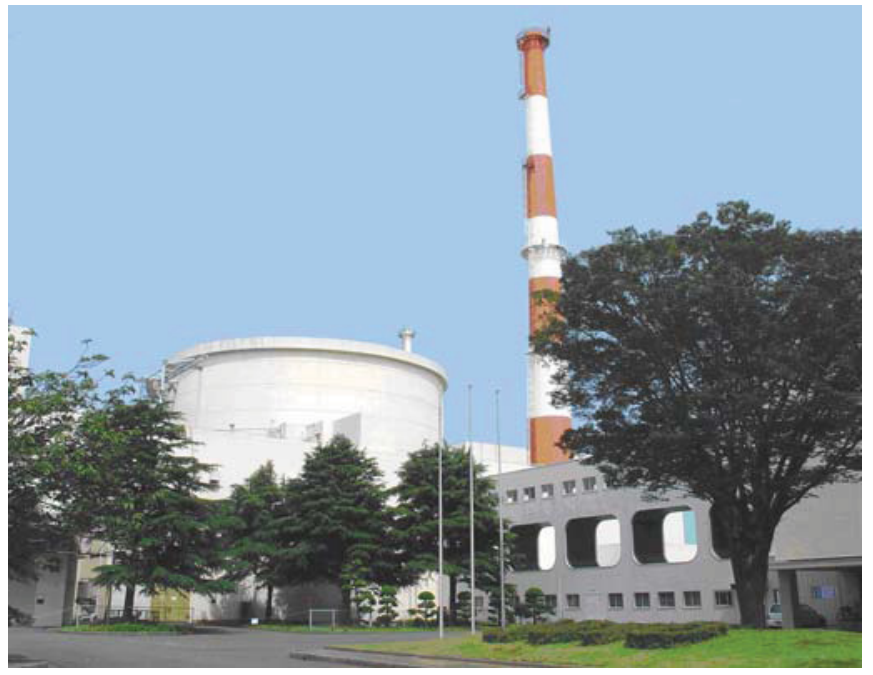

The Jōyō fast spectrum test reactor in Japan the JAEA. In 1984, the nuclear fuel cycle was successfully completed when plutonium extracted from reprocessing the spent fuel from Jōyō was reintroduced into the reactor. The major current role of Jōyō is to contribute to global fast reactor development through testing and technical exchanges with international researchers.

The reactor containment structure is approximately $175 \mathrm{ft}$ high and $90 \mathrm{ft}$ in diameter. It is made of carbon steel and contains the reactor vessel and the major components of the primary cooling system. The reactor vessel is about $12 \mathrm{ft}$ in diameter and $40 \mathrm{ft}$ high and made of stainless steel. The cover is a double rotating plug for refueling. Jōyō is not equipped with a generator for electricity production.

The core contains a maximum of 85 fuel assemblies, six control rods, and various irradiation test rigs. The fuel region is divided into two regions to flatten the neutron flux distribution. The fueled core is surrounded by reflectors and shielding subassemblies. Maximum reactor power is $140 \mathrm{MW}$ with fast neutron flux (greater than $0.1 \mathrm{MeV}$ ) of 4E15 neutrons per centimeter squared per second. 


\section{Monju}

Location: Tsurgura, Japan

Currently Supporting: Fast reactor demonstration

Status: Shutdown, repairs, and upgrades near completion

\begin{tabular}{|l|l|l|l|}
\hline Potential Program R\&D Applications are Shaded \\
\hline Light Water Reactors & $\begin{array}{l}\text { Irradiated Fuel } \\
\text { Separations }\end{array}$ & $\begin{array}{l}\text { Advanced Fuel } \\
\text { Development }\end{array}$ & Space Power Systems \\
\hline $\begin{array}{l}\text { High-Temperature } \\
\text { Reactor }\end{array}$ & Fast Spectrum Reactor & $\begin{array}{l}\text { Safeguards and } \\
\text { Security }\end{array}$ & $\begin{array}{l}\text { Modeling and } \\
\text { Simulation }\end{array}$ \\
\hline
\end{tabular}

Monju, a prototype fast breeder reactor in Fukui prefecture, near the town of Tsuruga, Japan, started up in 1994 to demonstrate fast reactor technology and operation. Thermal output is $714 \mathrm{MWt}$ and $280 \mathrm{MWe}$. There are 198 fuel assemblies, with each assembly containing 169 fuel pins. Inlet temperature is $397^{\circ} \mathrm{C}$ and outlet is $529^{\circ} \mathrm{C}$.

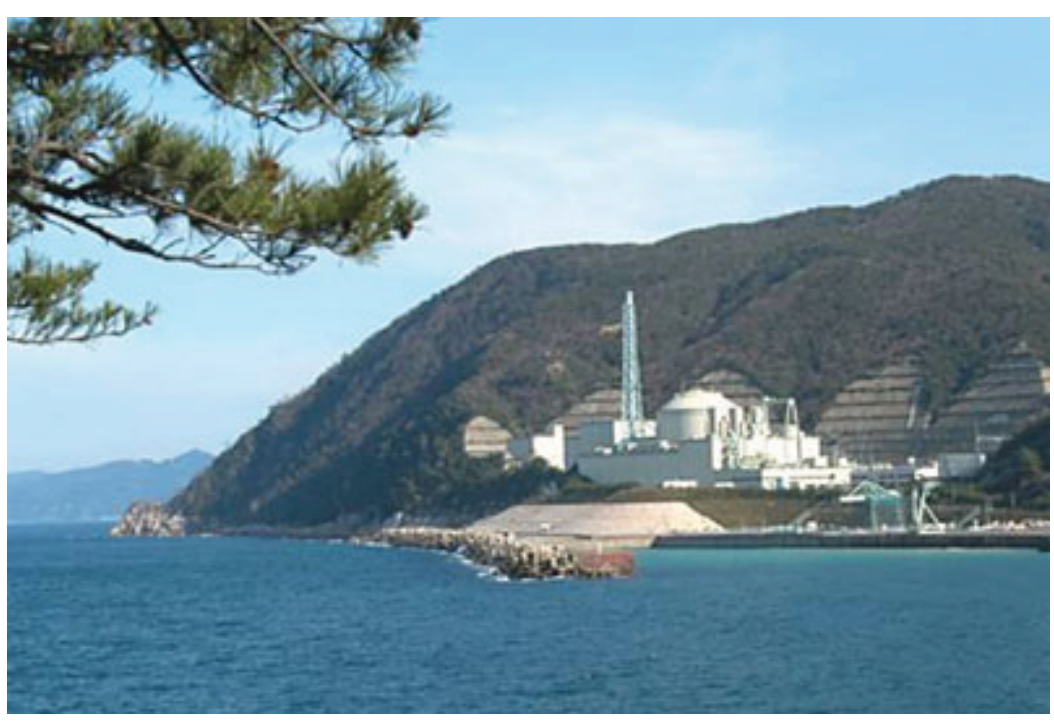

The reactor was shut down in 1995 after failure of a thermocouple well in the secondary circuit piping caused a small sodium leak and fire. The reactor has been idle for 11 years, but the Japanese Atomic Energy Association has maintained its viability. After the governor of Fukui formally approved preparations to restart the reactor 2 years ago, refurbishment has moved quickly and is near completion. The reactor is expected to restart in the fall of 2008 with upgraded leak-detection systems.

The Monju Reactor at Tsurgura, Japan 


\section{High-Temperature Engineering Test Reactor}

Location: Ōarai, Ibaraki, Japan

Currently Supporting: High-temperature gas reactor and process heat application testing

Status: Operating

\begin{tabular}{|l|l|l|l|}
\hline Potential Program R\&D Applications are Shaded & Space Power Systems \\
\hline Light Water Reactors & $\begin{array}{l}\text { Irradiated Fuel } \\
\text { Separations }\end{array}$ & $\begin{array}{l}\text { Advanced Fuel } \\
\text { Development }\end{array}$ & $\begin{array}{l}\text { Modeling and } \\
\text { Simulation }\end{array}$ \\
\hline $\begin{array}{l}\text { High-Temperature } \\
\text { Reactor }\end{array}$ & Fast Spectrum Reactor & $\begin{array}{l}\text { Safeguards and } \\
\text { Security }\end{array}$ \\
\hline
\end{tabular}

The High Temperature Engineering Test Reactor is a helium cooled graphite moderated test and demonstration reactor. It is used to demonstrate inherent safety features and develop high temperature gas reactor fuels, materials and technologies including nuclear heat applications such as hydrogen production. It began operation in 1998 and reached full power of $30 \mathrm{MW}$ with an outlet temperature of $850^{\circ} \mathrm{C}$ in December, 2001. The maximum reactor outlet coolant temperature of $950^{\circ} \mathrm{C}$ was achieved in April 2004. It is located at the Oarai Research and Development Center in Ōarai, Ibaraki, Japan.

The core is $2.9 \mathrm{~m}$ high and $2.3 \mathrm{~m}$ in diameter. TRISO coated fuel particles with $\mathrm{UO}_{2}$ kernel, about $6 \mathrm{wt} \%$

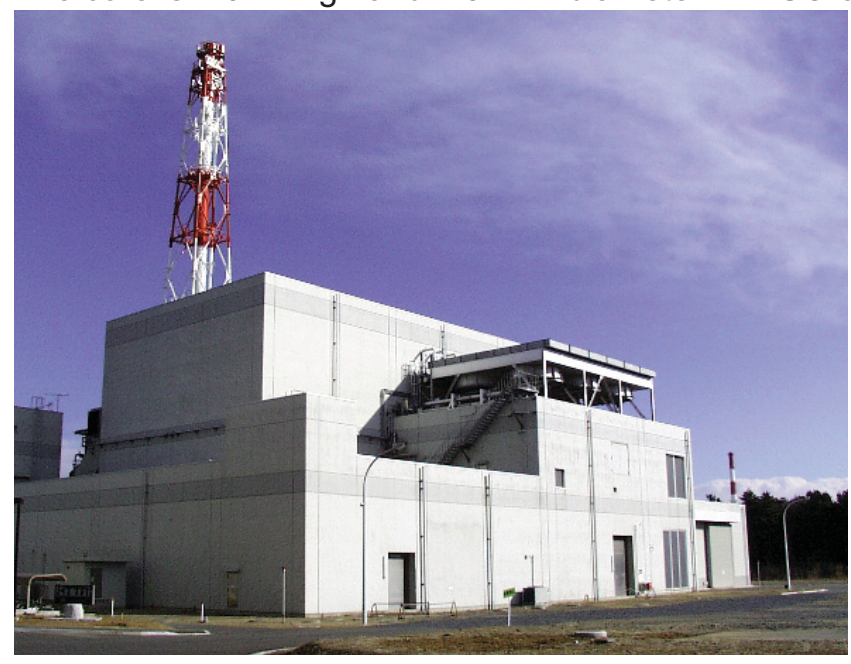

The High Temperature Engineering Test Reactor in Japan in average enrichment and $600 \mu \mathrm{m}$ in diameter, are dispersed in a graphite matrix and sintered to form a fuel compact. Fuel compacts are contained in a fuel rod, $34 \mathrm{~mm}$ in outer diameter and $577 \mathrm{~mm}$ in length. Fuel rods are inserted into vertical holes made in the graphite block and the graphite blocks are assembled into columns. Helium gas coolant flows through gaps between the holes and the rods. The active core consists of 30 fuel columns and seven control rod guide columns. These are surrounded by

12 replaceable reflector columns, nine reflector control rod guide columns and three irradiation test columns.

A primary core cooling system operates during normal operation and an auxiliary system is activated for residual heat removal following reactor scram. In case of accidents, however, no forced cooling is required because there is a vessel cooling system which has sufficient capacity to remove residual heat through natural convection and radiation.

Initial hydrogen production testing will utilize a steam reforming of methane process, but the final goal is to demonstrate hydrogen production from water without carbon dioxide emission. Hydrogen production tests are expected to begin in 2008. 


\subsubsection{Germany}

\section{Institute for Transuranium Elements}

Location: Karlsruhe, Germany

Currently Supporting: European Union—Joint Research Centre for Science and Technology

Status: Operational

\begin{tabular}{|l|l|l|l|}
\hline Potential Program R\&D Applications are Shaded \\
\hline Light Water Reactors & $\begin{array}{l}\text { Irradiated Fuel } \\
\text { Separations }\end{array}$ & $\begin{array}{l}\text { Advanced Fuel } \\
\text { Development }\end{array}$ & Space Power Systems \\
\hline $\begin{array}{l}\text { High-Temperature } \\
\text { Reactor }\end{array}$ & Fast Spectrum Reactor & $\begin{array}{l}\text { Safeguards and } \\
\text { Security }\end{array}$ & $\begin{array}{l}\text { Modeling and } \\
\text { Simulation }\end{array}$ \\
\hline
\end{tabular}

The Institute for Transuranium Elements serves international and commercial entities and the European Union as a reference center for basic actinide research. It supports nuclear fuel cycle safety, including waste management and safeguards systems and studies technological and medical applications of radionuclides/actinides. The Institute for Transuranium Elements focuses on methods for reducing the quantity and radiotoxicity of highly radioactive waste by separating out the long-lived nuclides and fabricating them into fuels and targets for transmutation. In addition, the Institute for Transuranium Elements is heavily involved in efforts to combat illicit trafficking of nuclear materials, and in developing and operating advanced detection tools to uncover clandestine nuclear activities. The Institute for Transuranium Elements also operates two onsite laboratories for the Directorate General Transport and Energy, Nuclear Inspection at the major European reprocessing plants: La Hague in France and Sellafield in England.
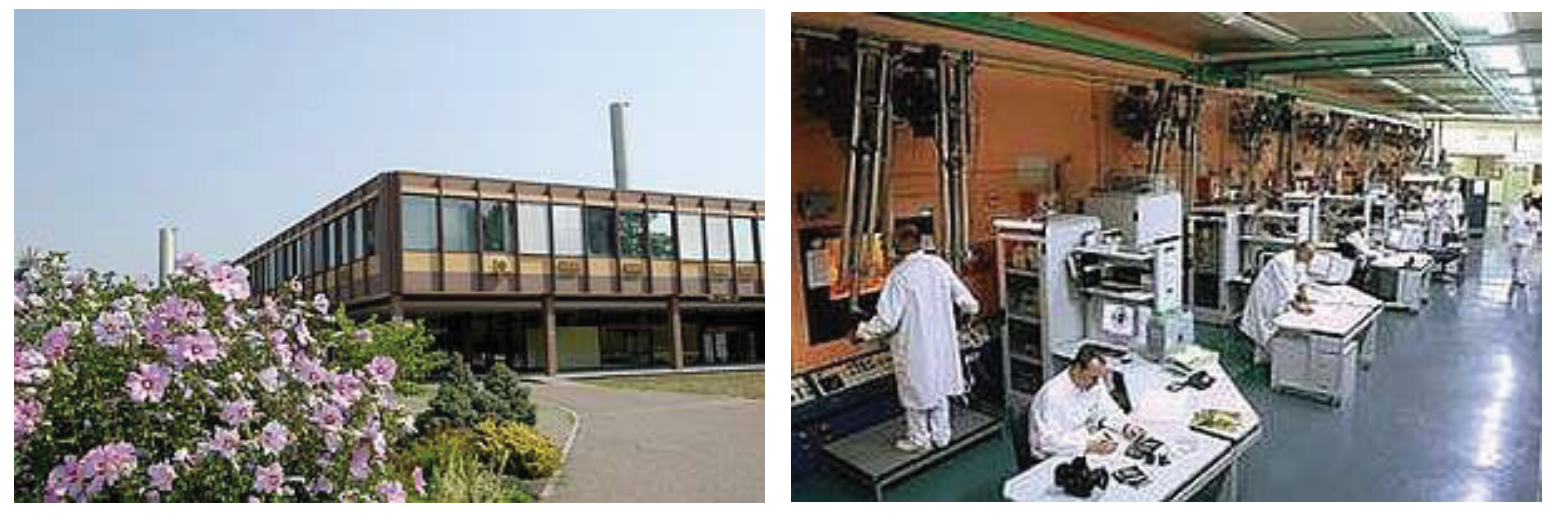

The Institute for Transuranium Elements at Karlsruhe, Germany 


\section{REFERENCES}

Report of the Infrastructure Task Force of the DOE Nuclear Energy Research Advisory Committee, 2003

Nuclear Facility Consolidation and Modernization Task Force Report - A Contractor Initiated Study, 2005

Hot Cells Strategy to Advance Nuclear Science and Engineering, 2006

DOE Complex-Wide Capability Report: (CWCR) Nuclear Energy Infrastructure, 2006

Inventory of Civilian Nuclear Facilities, Equipment, and other Assets, 2007

AFCF Existing Facilities Data Report (DRAFT), 2008

U.S. Facilities Needed to Support Global Nuclear Energy Partnership Research and Development (DRAFT), 2008

SFR-Related Facilities (United States, France, and Japan) DRAFT, 2008

Nuclear Energy for the Future: Required Research and Development Capabilities-An Industry Perspective, 2008

Executive Recommendations for Nuclear R\&D Capabilities, 2008

Note: Facility descriptions and program and infrastructure data also were gathered from applicable web sites. 


\section{APPENDICES}

5.1 Nuclear Energy for the Future: Required Research and Development Capabilities-An Industry Perspective

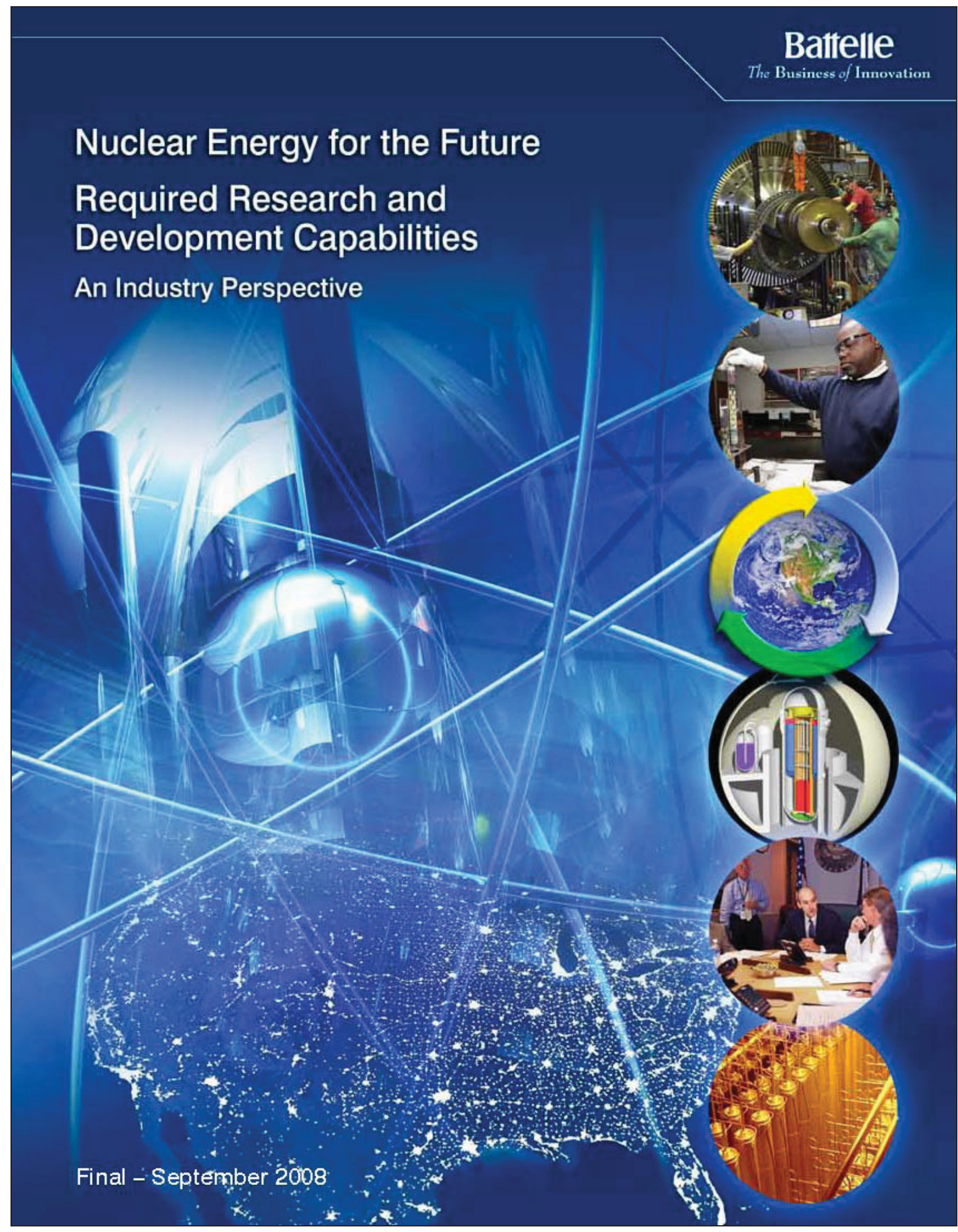




\title{
Participant Organizations
}

A total of 35 organizations (including industry, universities, regulators, national laboratories and others) participated in the development of this analysis by offering input, ideas, and/or draft reviews.

\author{
Industry \\ AREVA NP \\ Bechtel Corporation \\ Dominion \\ Electric Power Research Institute \\ Entergy \\ Exelon Corporation \\ FirstEnergy Nuclear Operating Company \\ General Electric Hitachi Nuclear Energy \\ Global Nuclear Fuel, LLC \\ Nuclear Energy Institute \\ Southern Nuclear Operating Company, Inc. \\ The Babcock \& Wilcox Company - Technical Services Group \\ UniStar Nuclear Energy \\ URS Corporation - Washington Division \\ Westinghouse Electric Company, LLC \\ Universities \\ Georgia Institute of Technology \\ Idaho State University \\ Massachusetts Institute of Technology \\ North Carolina State University \\ Oregon State University \\ The Ohio State University \\ University of California, Berkeley \\ University of Florida \\ University of Idaho \\ University of Michigan \\ University of New Mexico \\ National Laboratories \\ Argonne National Laboratory \\ Idaho National Laboratory \\ Los Alamos National Laboratory \\ Oak Ridge National Laboratory \\ Pacific Northwest National Laboratory \\ Savannah River National Laboratory \\ Others \\ Battelle \\ Marston Consulting
}

This analysis is intended to represent the synthesis of data provided and should not be viewed as representing any particular organization's interest nor does organizational participation indicate endorsement. 


\section{Table of Contents}

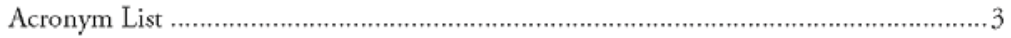

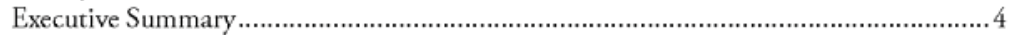

Section 1 - Perspective .........................................................................................................6

Section 2 - Nuclear Energy Industry Goals 2010 to 2050........................................9

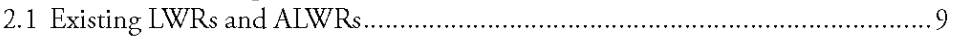

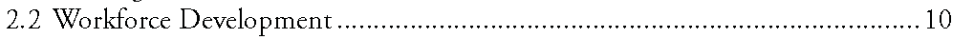

2.3 Sustainable Fuel Cycle …………................................................................ 11

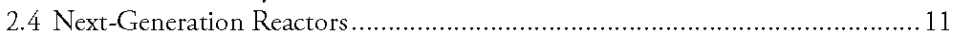

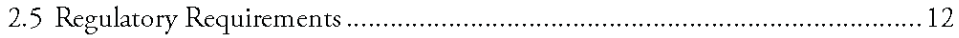

2.6 Safeguards and Security …....................................................................... 12

Section 3 - Identify and Close the Required Capability Gaps.................................13

3.1 Process to Identify and Close Capability Gaps.............................................. 13

3.2 Actions Needed to Close Required Capability Gaps ...................................... 16

Section 4 - Required Nuclear Energy R\&D Facilities ..................................................18

4.1 Identification of Facility Requirements......................................................... 18

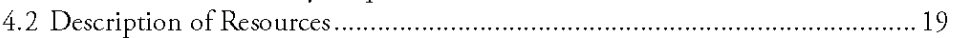

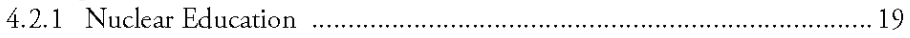

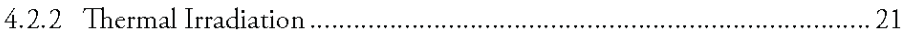

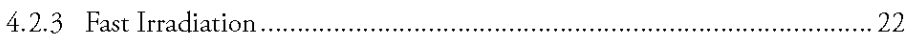

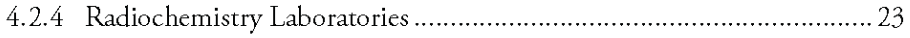

4.2.5 Hot Cells for Separations ................................................................ 24

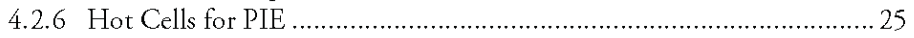

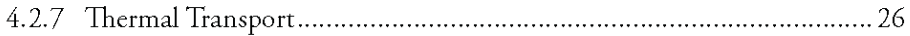

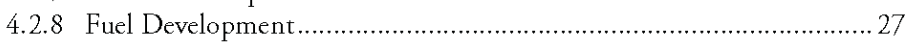

4.2.9 Licensing Demonstration for an HTR............................................... 28

4.2.10 Licensing Demonstration for a Fast Reactor ....................................... 29

4.2.11 Specialized Engineering Development Laboratories............................ 30

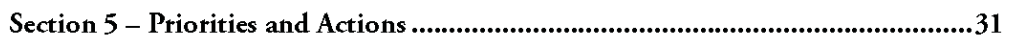

Section 6 - Conclusions and Benefits...................................................................33

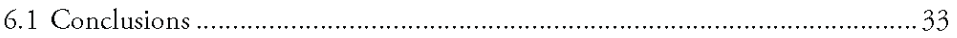

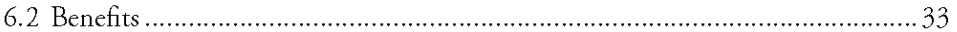

Appendix A - Process to Identify Capability Requirements.......................................34

A.1 Methods Used to Gather Input ................................................................. 34

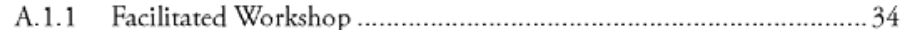

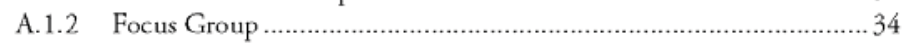

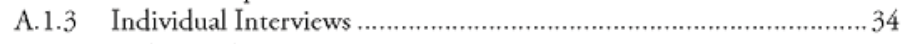

A.1.4 Web-Based Focus Group............................................................... 34

A.2 Framework Used to Gather Input …….............................................. 34

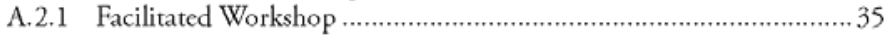

A.2.1.1 Current State/Future State/Gap Identification ........................35

A.2.1.2 Prioritization Process........................................................... 35

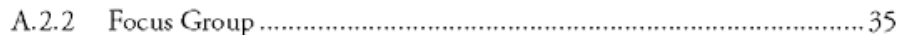


A.2.2.1 Current State/Future State/Gap Identification........................ 35

A.2.2.2 Prioritization Process ........................................................... 35

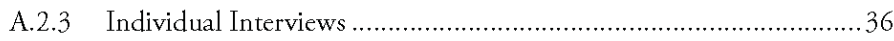

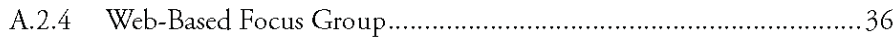

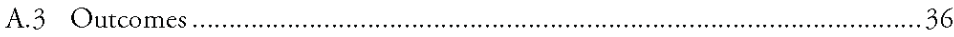

Attachment A-1 Nuclear Energy R\&D Cabilities Outlook

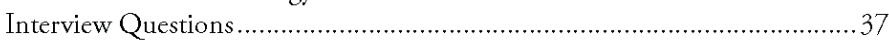

Appendix B - Workshop/Focus Group Summaries .............................................38

Appendix C - Required Capabilities Derived From Other Industries...................45

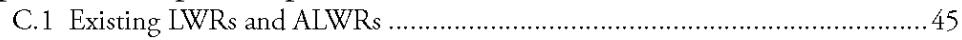

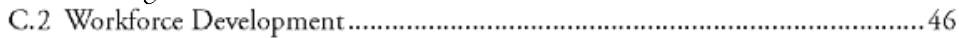

C.3 Sustainable Fuel Cycle............................................................................ 46

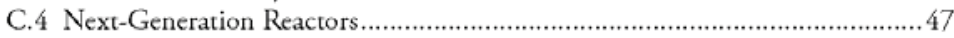

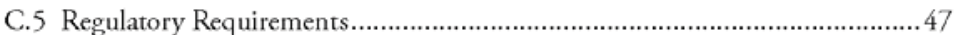

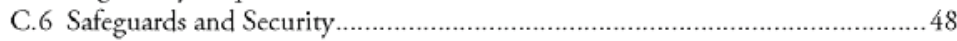

\section{List of Tables}

Table 3-1. Required Priority Capabilities for 2010 to 2050.

Table 3-2. Sources of Required Capabilities to Meet Nuclear Energy Industry

Goals in the 2010 to 2050 Time Frame.....

Table 4-1. Matrix of Necessary Nuclear-Unique Capabilities and

Required Resources.

Table 5-1. Summary of Nuclear R\&D Facility Priorities.

Table B-1. Light Water Reactors/Advanced Light Water Reactors Focus Areas — Capability Priorities ...............................................................39

Table B-2. Workforce Development Focus Area - Capability Priorities.......................40

Table B-3. Sustainable Fuel Cycle Focus Area - Capability Priorities .......................... 41

Table B-4. Next-Generation Reactors Focus Area-Capability Priorities.................... 42

Table B-5. Regulatory Requirements Focus Area — Capability Priorities......................43

Table B-6. Safeguards and Security Focus Area —Capability Priorities..........................44

\section{List of Figures}

Figure 1-1. Top-Down/Bottom-Up Processes for Developing a Rank-Ordered Nuclear Energy Facilities Program 8

Figure 3-1. Capability Gap-Closure Flow Chart ………............................................ 15 


\section{Acronym List}

\begin{tabular}{|c|c|c|c|}
\hline ALWR & Advanced Light Water & INL & Idaho National Laboratory \\
\hline \multirow{3}{*}{ ANL } & Reactor & INPO & Institute of Nuclear Power \\
\hline & Argonne National & & Operations \\
\hline & Laboratory & K-12 & Kindergarten Through 12 th Grade \\
\hline ATR & Advanced Test Reactor & kWh & Kilowatt Hour \\
\hline${ }^{\circ} \mathrm{C}$ & Degree Celsius & & \\
\hline \multirow[t]{2}{*}{ CFR } & Code of Federal & LMR & Liquid Metal Reactor \\
\hline & Regulations & LTA & Lead Test Assembly \\
\hline $\mathrm{CO}_{2}$ & Carbon Dioxide & LWR & Light Water Reactor \\
\hline DBT & Design Basis Threat & MC\&A & Material Control and \\
\hline DOE & Department of Energy & & Accountability \\
\hline \multirow[t]{2}{*}{ DOE NE } & DOE Office of Nuclear & MMBTU & Million British Thermal Units \\
\hline & Energy & MOX & Mixed Oxide \\
\hline EPA & Environmental Protection & MWth & Megawatt Thermal \\
\hline \multirow[t]{2}{*}{ EPRI } & $\begin{array}{l}\text { Agency } \\
\text { Electric Power Research }\end{array}$ & NANTeL & $\begin{array}{l}\text { National Academy for Nuclear } \\
\text { Training e-Learning }\end{array}$ \\
\hline & Institute & $\mathrm{NE}$ & Nuclear Energy \\
\hline \multirow{3}{*}{$\begin{array}{l}\text { FFTF } \\
\text { GNEP }\end{array}$} & Fast Flux Test Facility & NEAC & Nuclear Energy Advisory \\
\hline & Global Nuclear Energy & & Committee \\
\hline & Partnership & NEI & Nuclear Energy Institute \\
\hline \multirow[t]{2}{*}{ GWd/MTU } & Gigawatt Days per Metric & NGNP & Next-Generation Nuclear Plant \\
\hline & Ton of Uranium & NGR & Next-Generation Reactors \\
\hline HFEF & $\begin{array}{l}\text { Hot Fuel Examination } \\
\text { Facility }\end{array}$ & NRC & Nuclear Regulatury Commission \\
\hline \multirow{4}{*}{$\begin{array}{l}\text { HFIR } \\
\text { HLW } \\
\text { HTGR }\end{array}$} & High Flux Isotope Reactor & NSF & National Science Foundation \\
\hline & High-Level Waste & ORNL & Oak Ridge National Laboratory \\
\hline & High-Temperature Gas- & PBMR & Pebble Bed Modular Reactor \\
\hline & $\begin{array}{l}\text { cooled Reactor } \\
\text { colose }\end{array}$ & PIE & Post-Irradiation Examination \\
\hline \multirow{3}{*}{$\begin{array}{l}\text { HTR } \\
\text { HTTR }\end{array}$} & High-Temperature Reactor & ppm & Parts Per Million \\
\hline & High-Temperature Test & $\mathrm{R} \& \mathrm{D}$ & Research and Development \\
\hline & Reactor & REU & Research Experience for \\
\hline \multirow[t]{2}{*}{$\mathrm{I} \& \mathrm{C}$} & Instrumentation and & & Undergraduates Program \\
\hline & Control & $S \& S$ & Safeguards and Security \\
\hline \multirow[t]{2}{*}{ IAEA } & International Atomic & SFC & Sustainable Fuel Cycle \\
\hline & Energy Agency & SSC & Systems, Structures, and \\
\hline \multirow[t]{2}{*}{ IHX } & Intermediate Heat & & Components \\
\hline & Exchange(r) & $\mathrm{UCO}$ & Uranium Oxycarbide \\
\hline \multirow[t]{3}{*}{ IMET } & Irradiated Materials & U.S. & United States \\
\hline & Examination and Testing & USD & U.S. Dollars \\
\hline & Laboratory & $\mathrm{V} \& \mathrm{~V}$ & Validate and Verify \\
\hline
\end{tabular}




\section{Executive Summary}

Nuclear energy has been prowen safe, reliable, and affor dable. Nuclear energy is poised to play an ewer-increasing role in rreeting future energy de rrand and in managing carbon emissions. In response to this opportunity, the nuclear energy industry, together with the U.S. Departrrent of Energy (DOE), is extending the service life of the currently operating reactors, de ploying actuanced light water reactors (AL MPs), exploring applications beyondelectricity production, de veloping nextgeneration reactors, and taking steps to close the nuclear fuel cycle. Essential research and dewelopment (R\&D) capabilities and facilities are required to achieve these goals. Only a portion of the require d capabilities and facilities are currently available.

Pecognizing these needs, the DOE Office of Nuclear Energy (DOE NE) requested Battelle to coordinate with the domestic nuclear energy industry and the acaderric community to identify the capabilities and facilities require d to support the achieverrent of the nuclear energy industry's goals. Battelle designed and led a fourstep process to identify the require dcapabilities and facilities. The first step obtained extensive input from the industry and acade rric communities to define goals for the 2010 to 2060 tirre frame across six key focus areas - the existing light water reactors (LMPS) and ALWRs, workforce dewelopment, the establishment of a sustainable fuel cycle, development of next-generation reactors, regulatory requirements, and safeguards and security. The second step identified and prioritized nee ched capabilities. The third step identified the gaps between available capabilities and the requirements to fill those gaps. The fouth step identified the types of facilities and resources nee ded to provide the necessary R\&D capabilities.

This multistep process ickntifie d rany capabilities that will be key to achieving nuclear energy goals, including ensuring the reliability of plant systerns, structure, and component materials through the plant's extende d lifetime; optimizing training through greater use of technical training centers, new methods, and improved skill and aptitude assessment tools; developing recycling technologies that are economically competitive, are more resistant to proliferation, and minimize waste disposal impact; and enhancing cybersecurity capabilities to ensure the safety and security of plant systerrs. Although many of the required capabilities exist or are uncker dewelopment in other industries, several capabilities are unique to the nuclear energy industry and require specialized facilities. Such facilities include radiochemistry laboratories, hot cells for post-irradiation examination and radionuclide separations, fuel developrrent laboratories, specialized engineering de weloprrent laboratories, and prototype reactors for licensing demonstrations.
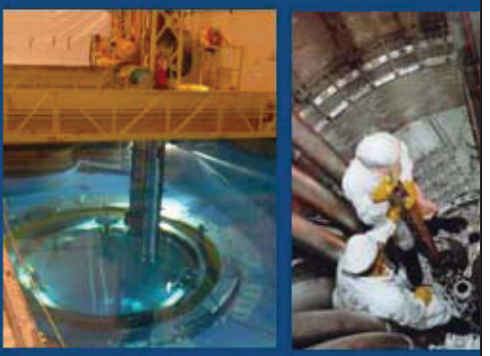

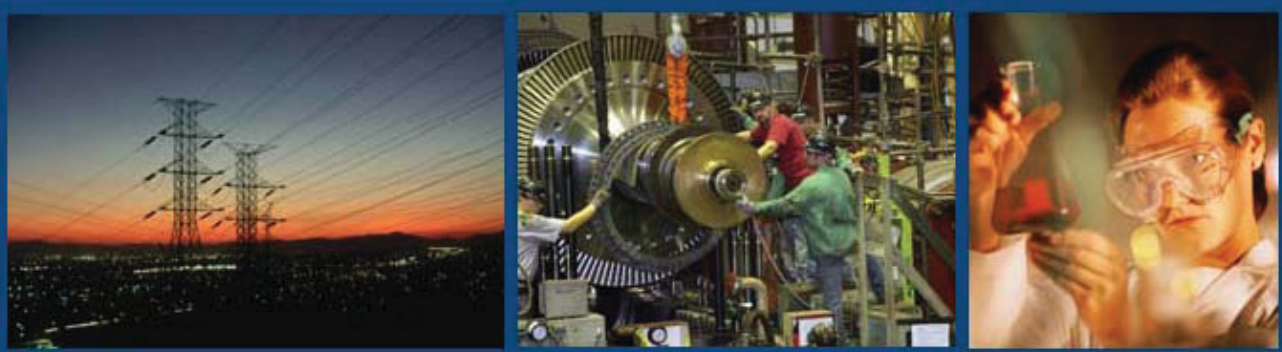

Action is required to provide the $R \& D$ capabilities and facilities unique to nuclear energy in order to ac hieve the domestic industry's goals identified for the 2010 to 2050 tirne frarre. This analysis identifies five priority R\&D facilities including three new facilities. The new facilities are as follows: (1) the High-Temperature Reactor Licensing Dernonstration, (2) the Fuel Oycle R\&D facilities, and (3) the Fast Reactor Licensing Dernonstration. In addition, a further evaluation of neece dinvestrrent in Nuclear Education Facilities is required. Nuclear facilities require significant capital investrnent and substantial annual resources to operate and maintain. Facilities currently available in the U.S. DOE complex could satisty many of the requirements, providing a bridge to the new facilities nee cled. Howewer, the available facilities fall short of providing much of the critical capabilities required to achieve the nuclear energy industry's long-range goals. In addition, given the age andoriginal purpose of the existing facilities, many of the U.S. DOE facilities require additional inwestment for improwernents and nee ded modifications. To address these needs, it is recorrmended that the DOE NE establish the Strategic Nu clear Energy Capability Initiative, a multiyear, user-drinen initiative to provide the required R\&D capabilities and facilities. It is recognized that the cost of providing the required capabilities and facilities is significant. However, the benefits realized in terms of energy security and managing carbon emissions are enormous.

This analysis generate $d$ the following conclusions:

1. A robust workforce must be available for the domestic nuclear energy industry to continue with its proven record of de livering baseload electric ity in a safe, reliable, and cost-effective manner.

2. The nuclear energy industry has established rreaningful goals for the future These goals include extending the service life of the currently operating reactors, de ploying ALMRs, closing the fuel cycle, and developing next-generation reactors leading to new applications for nuclear energy.

3. Essential R\&D capabilities and facilities are required to erable the industry to achieve these goals. Through the establishment of a multiyear, user-driven Strat egic Nuclear Energy Capability Initiative, the DOE NE can provide the nee cled capabilities and facilities.

4. Successful establishment of the Initiatiue will also provide the DOE NE with the necessary foundation to build public-private partnerships and international collaboration to facilitate provision of the needed capabilities and facilities. 


\section{Section 1 - Perspective}

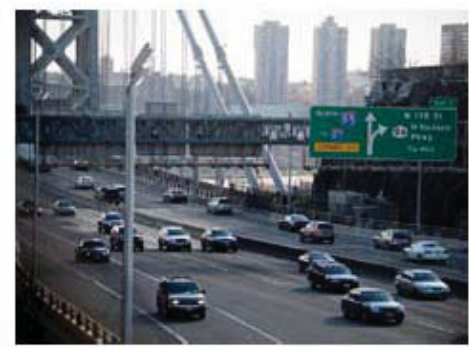

We cannot rely on "silver bullets" to address either the national (or global) energy supply/demand situation or to manage global climate change in the next 50 years. The only pragmatic solution to either of these critical issues is a robust portfolio approach with a balance of energy sources. In all reasonable forecasts, nuclear energy must play an ever-increasing role in the generation of electricity, which accounts for roughly one-third of the global, man-made carbon dioxide $\left(\mathrm{CO}_{2}\right)$ emissions. However, to deal effectively with the combined threat of climate change and energy security, the role of nuclear energy must be expanded beyond generating electricity, to providing $\mathrm{CO}_{2}$ emissionfree process heat for chemical plants and refineries, and developing nonconventional, indigenous hydrocarbon sources. Eventually, nuclear energy will produce hydrogen from water to provide transportation fuels and enable the production of gases and liquids from our most abundant nonconventional hydrocarbon-coal-without the emission of large quantities of $\mathrm{CO}_{2}$. A sidebar discussion on the following page provides an energy and environmental perspective for this report.

The U.S. Department of Energy (DOE), in particular, the Office of Nuclear Energy (DOE NE), must be a key driver in the continuing development of energy from fission. The nuclear energy industry's prioritized areas of focus, as articulated by the Nuclear Energy Institute (NEI), include the following:

- Safe and economic operation of the current fleet of 104 nuclear plants for as long as possible - to 80 years and beyond

- Introduction of new advanced light water reactors ( $A L W R$ S) through a manageable licensing process with reasonable financial risks

- Development and demonstration of a new generation of nuclear energy sources that will provide a useful range of emission-free process heat (250 degrees Celsius $\left[{ }^{\circ} \mathrm{C}\right]$ to $750^{\circ} \mathrm{C}$ ) and higher temperatures $\left(950^{\circ} \mathrm{C}\right)$ for efficient production of hydrogen and oxygen from water

- Closure of the nuclear fuel cycle to assure a reliable source of future nuclear fuel that has no undue proliferation risk and that minimizes the burden on deep geological disposal.

These focus areas require access to existing capabilities and development of new capabilities. Some existing capabilities that are common in other industries include automation, advanced instrumentation and control, knowledge management, highcapacity computing, and nano-materials. Existing capabilities that are needed by only a limited subset of other industries include automated welding and inspection techniques, training and simulation of operation, and heavy component construction. However, 


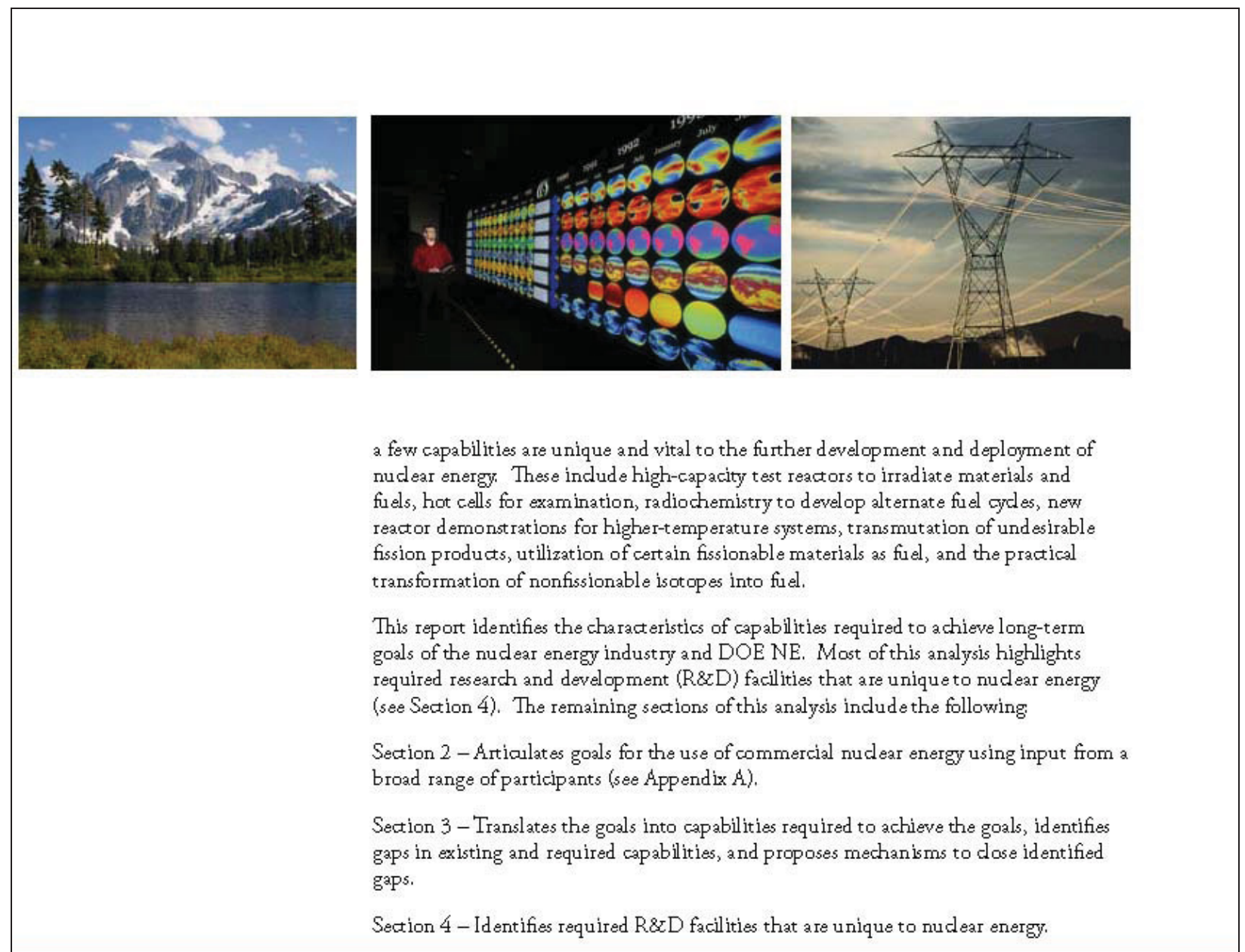

Energy and Environment - A Future View

Approaching the middle of the 21 st century, the price (and availability) of natural gas has reached a level of $\$ 12$ U.S. dollars (USD) (2008) per million British Thermal Units (MMBTU), making it too expensive to burn as an industrial fuel and limiting its uses to chemical fee dstock and household heating. The price of carbon has reached a level of over $\$ 40$ USD (2008) per metric ton. Eken with strong meas ures to increase the efficient use of electricity. the demand in 2050 is expected to be double that in 2010 . Carbon capture and sequestration is a viable, but expensive, approach to reducing $\mathrm{CO}_{2}$ emissions from fossil-fired power plants. Electricity from "renewable" generation has reached a record 25 percent in 2045 with the advent of large wind machines and affor dable solar panels using thir-film technologies. Geothermal dewelopments are increasing each year. Technology makes it possible to extract almost 40 percent of the potential energy from each pound of uranium, and the volume of vitrified waste being placed into deep geological disposal is a fraction of what was thought possible in 2010 . With the advent of new extremely low emission generation technologies, an atmospheric $\mathrm{CO}_{2}$ concentration of 450 parts per million ( $(\mathrm{ppm})$ is deemed to be practical within 30 years.

Oil has stayed at an inflation-corrected $\$ 130$ USD per barrel for some time. Oil imports are down to a record low of 10 percent. The use of nuclear energy to prowide reliable process heat and hydrogen is growing steadily. The earliest nuclear power plants are finally being decommissioned after 80 years of safe operation. Electricity and hydrogen now account for roughly one-half of automotive fuel. Bio-diesel and nonconventional hydrocarbonderiwed diesel fuel all truck and rail trans port. 

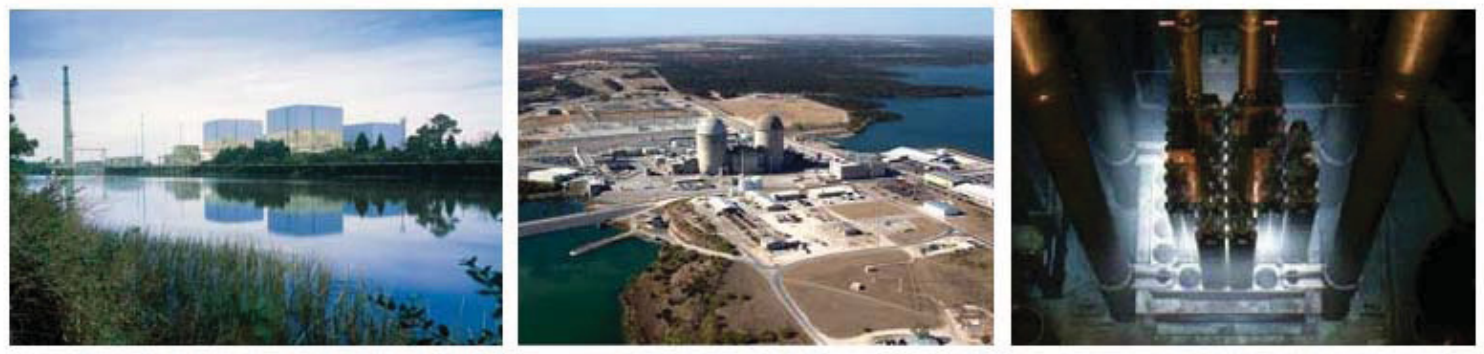

Section 5-Identifies recommended priorities and actions.

Section 6 - Provides a nalysis conclusions and a statement of expected benefits.

Appendices A, B, and C-Outline the process used for the 2010 to 2050 nuclear energy industry goal development, identification of required capabilities, and required capabilities transferred from or devel oped with other industries.

This analysis represents one phase of a three-phase process designed to develop a DOE Nuclear Energy Plan to ensure the highest priority facilities and staff are available through 2050 to meet the key objectives of the nuclear energy industry, as shown in Figure 1-1. Another phase is a parallel effort led by Idaho National Laboratory (INL) to assess the condition and readiness of aurrent domestic and international facilities that could be used to provide the needed capabilities id entified in Section 3 of this report. The final phase is to integrate the outputs of this "top-down" study and the "bottomup" INL effort to develop a prioritized list of capabilities required to meet the long-term goals of the nuclear energy industry.

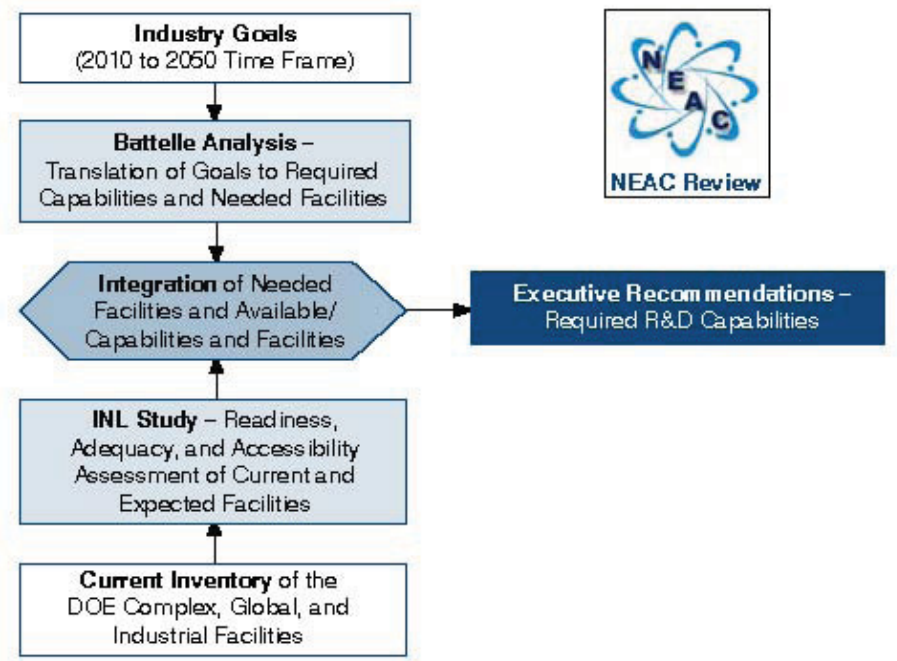

Figure f-f. Top-Down/Bottom-Up Processes for Developing a Rank-Ordered Nuclear Energy Facilities Program (Nuclear Energy Advisory Committee [NEAC] review provided in the blice-colored stepsi) 

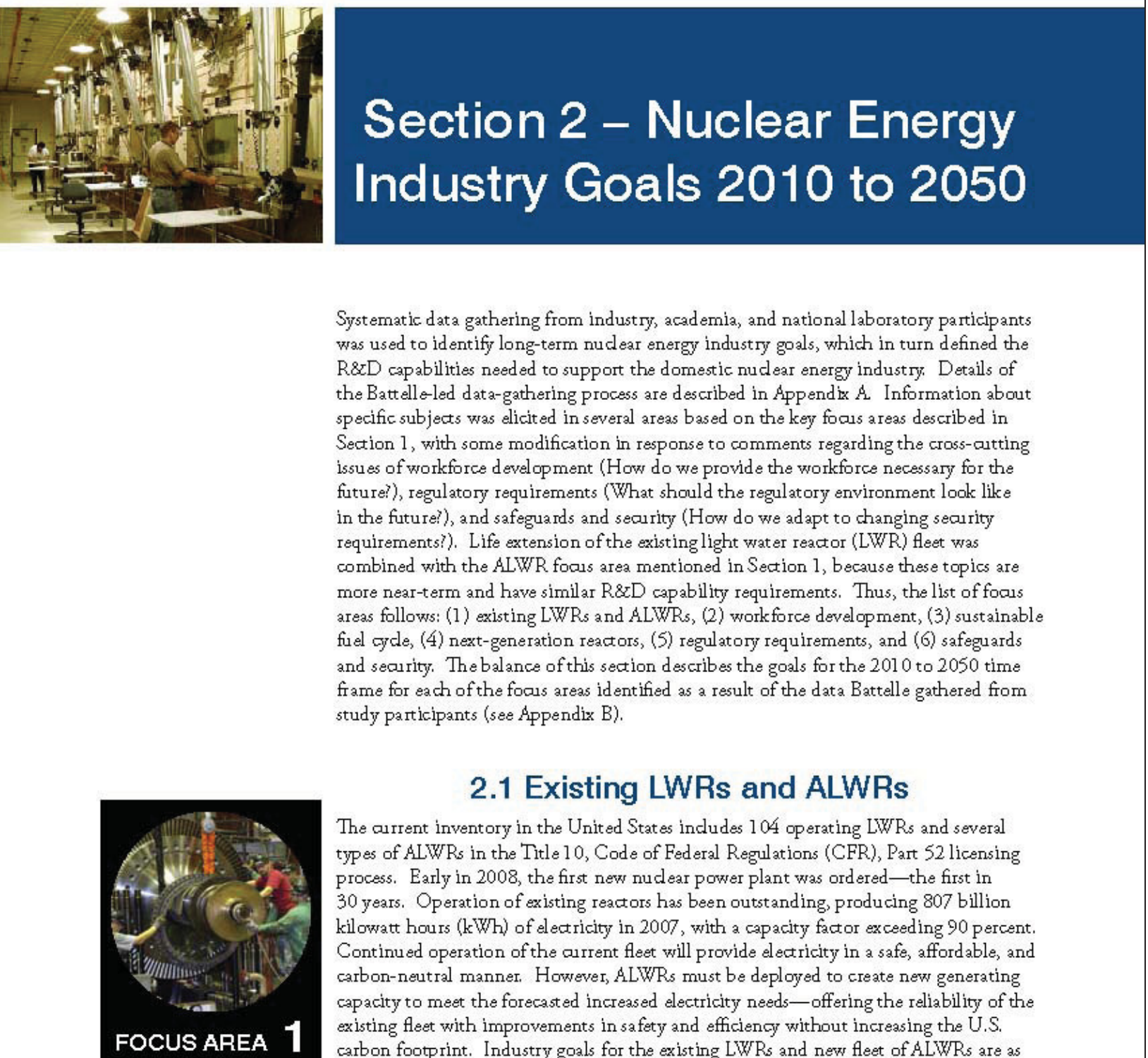

Systematic data gathering from industry, academia, and national laboratory participants was used to identify long-term nuclear energy industry goals, which in turn defined the $\mathrm{R} \& \mathrm{D}$ capabilities needed to support the domestic nuclear energy industry. Details of the Battelle-led data-gathering process are described in $A_{\text {ppendix }} A$ Information about specific subjects was elicited in several areas based on the key focus areas described in Section 1, with some modification in response to comments regarding the cross-autting issues of workforce devel opment (How do we provide the workforce necessary for the future?), regulatory requirements (What should the regulatory environment look like in the future?), and safeguards and security (How do we ad apt to changing security requirements?). Life extension of the existing light water reactor (LWR) Heet was combined with the ALWR foas area mentioned in Section 1, because these topics are more near-term and have similar R\&D capability requirements. Thus, the list of focus areas follows: (1) existing LWRs and ALWRs, (2) work force development, (3) sustainable fuel cycle, (4) next-generation reactors, (5) regulatory requirements, and (6) safeguards and seaurity. The balance of this section describes the goals for the 2010 to 2050 time frame for each of the focus areas identified as a result of the data Battelle gathered from study participants (see Appendix B).

\subsection{Existing LWRs and ALWRs}

The aurrent inventory in the United States includes 104 operating LWRs and several types of ALWRs in the Title 10, Code of Federal Regulations (CFR), Part 52 licensing process. Early in 2008, the first new nuclear power plant was ordered-the first in 30 years. Operation of existing reactors has been outstanding, producing 807 billion kilowatt hours (kWh) of electricity in 2007 , with a capacity factor exceeding 90 percent. Continued operation of the current fleet will provide electricity in a safe, affordable, and carbon-neutral manner. However, ALWRs must be deployed to create new generating capacity to meet the forecasted increased electricity needs - offering the reliability of the existing fleet with improvements in safety and efficiency without increasing the U.S. carbon footprint. Industry goals for the existing LWRs and new fleet of ALWRs are as follows:

\section{Existing LWRs and ALWRs}

- Extend the lifetime of the aurrent fleet to "life beyond 60 " years while maintaining the aurrent excellent safety record and high reliability (greater than 90 percent average fleet capacity factor).

- Build and deploy an additional 100 to 250 ALWRs. Using lessons learned from plant construction, evolve design and construction techniques to reduce schedules and costs. Design plants for reduced water consumption. 
- Address material aging in existing reactors by implementing optimal aging management programs for:

- Major pressure-retaining components and core internal structures

- Key support systems, structures, and components (SSC), including cabling and concrete structures.

- Improve current fuel types to el iminate leaking fuel pins. Develop new fuel/clad combinations that increase burnup to 100 gigawatt days per metric ton of uranium (GWd/MTU).

- Replace existing analog instrumentation and control (I\&C) systems with digital systems that can be upgraded as technology advances-with full regulatory acceptance where appropriate.

- Develop prognostic equipment that optimizes maintenance programs and also provides condition status reports to plant operators.

\subsection{Workforce Development}

In the 2010 to 2050 time frame, the nuclear energy industry will require a new work force to operate both the existing fleet of reactors as well as new advanced reactors. Industry organizations (e.g., NEI, Electric Power Research Institute [EPRI]) aurrently are prediating significant shortfalls in trained operations staff, design engineers, construction workers, technicians, radiation protection personnel, and related technical staff. Aggressive near-term steps must be taken to ensure availability of a fully trained workforce. Goals identified by the nuclear energy industry are as follows:

- Establish a robust pipeline of new staff at all levels for all skill sets.

- Actively develop the next generation of engineers by promoting DOE, EPRI, and Nuclear Regulatory Commission (NRC) scholarship/fellowship programs that encourage students to enter nuclear energy fields at both the undergraduate and graduate levels to supply industry and government needs.

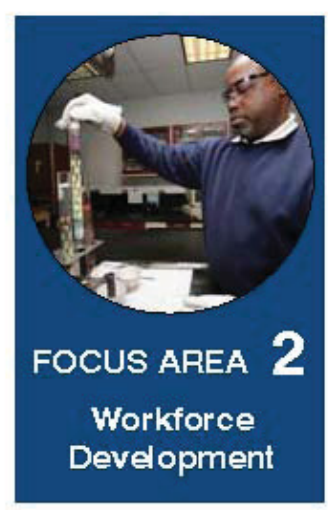

- Encourage a fresh, relevant advertising campaign, capitalizing on the current high level of awareness and interest in energy. Highlight the strategic importance of the nuclear energy industry and the career opportunities available to meet some of the nation's biggest challenges.

- Encourage collaboration among universities, national laboratories, and industry to leverage infrastructure and enhance the skills of the entering workforce-including implementation of impactul rotational assignments to provide more effective education and training opportunities.

- Facilitate students entering technical fields of study through effective K-12 programs in collaboration with other industry and government initiatives (e.g., science, technology, engineering, and math education).

- Develop new technologies to capture exiting workforce knowledge and integrate this information into training programs, reference databases, and knowledge transfer tools. Retrain staff for new jobs where appropriate (including displaced staff from other industries) —improving staff retention rates.

- Deploy centralized, standardized, and cost-effective regional training centers for staff. Provide remote training tools for qualification, such as the Institute of Nuclear Power Operations' (INPO's) National Academy for Nuclear Training e-Learning (NANTeL) information system. 


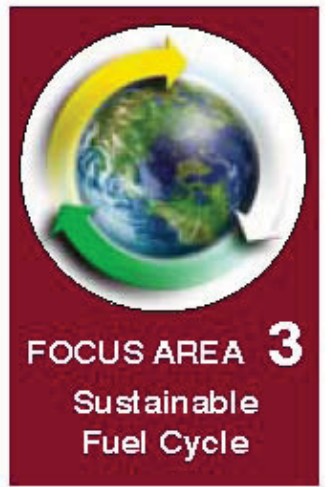

\subsection{Sustainable Fuel Cycle}

The current fuel cycle is open and uses only a few percent of the potential energy in uranium. Used nuclear fuel is discharged and cooled; eventually, it will be deposited into a deep geologic repository. The proposed repository at Yucca Mountain will reach its planned regulatory limit within three years, based on the aurrent inventory and generation rate of used fuel. The fuel can be safely stored outside the repository for at least 100 years. Under the Nudear Waste Policy Act, the DOE is obligated to take title of the used fuel and provide for its disposition. The nuclear energy industry is anxious for $D O E$ to fulfll its responsibility.

Most of the "waste" in used fuel consists of dadding hardware, and uranimm. The troublesome elements - those that have long half-lives and must be managed for long times in the repository-contribute only about 3 to 4 percent of the total mass. By separating the various components of used nuclear fuel, it would be possible to concentrate the "difficult-to-deal-with" materials into much smaller volumes, effectively lengthening the operating life of the repository. A sustainable fuel cycle would use a significantly greater portion of the available energy. Ad equate natural uranium resources are projected to be available to support the anticlpated growth of nuclear energy in the United States through mid-century. The price of uranium will likely increase as the supply of easily recoverable uranium is reduced.

Industry desires the establishment of a sustainable fuel cycle, including the timely removal of used fuel from the reactor. The fuel can be recycled to ensure an affordable supply of uranium, and more compact waste for ms can be developed. Industry needs a sustainable fuel cycle one that provides reliable fuel at a competitive price. The goals identified for such a sustainable fuel cycle are as follows:

- Ensure that a seaure, cost-effective, sustainable fuel supply exists.

- Clase the fuel cycle through the recycling of used fuel in a cost-effective manner.

- Ensure that adequate deep geological disposal for high-level waste (HLW) and used fuel exists, with retrievability maintained for 100 years.

- Provide advanced safeguards to ensure control of the materials recovered from fuel recycling.

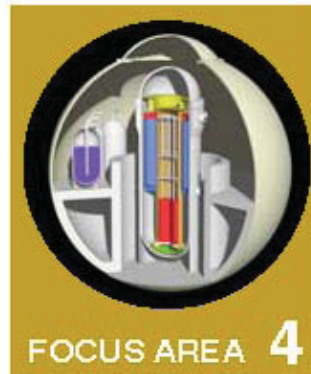

\subsection{Next-Generation Reactors}

The nuclear energy industry (including electric utilities and oil, gas, and chemical companies) has identified key roles in nontraditional areas for advanced reactor systems to significantly contribute to the nation's energy needs with increased security of supply. These areas include high-temperature reactors (HTRs) to supply process heat and hydrogen to oil refineries and chemical plants for the development of nonconventional hydrocarbon resources (i.e, oil sands and oil shale deposits). Additionally, HTRs, coupled with coal-to-liquids and coal-to-gas plants will allow the utilization of coal, our largest domestic hydrocarbon resource, to produce feedstock for refineries and chemical plants with essentially no $\mathrm{CO}_{2}$ emissions. Smaller, grid-appropriate reactors could be deployed to areas of the country and the world that cannot support large ALWRs. Finally, fast reactors could be devel oped to transmute materials to reduce waste toxicity and enhance the use of uranium. The specific goals identified by industry are as follows:

- Use advanced reactors for process heat generation and/or hydrogen production.

- Develop and deploy grid-appropriate reactors.

- Develop and implement fast reactors to support fuel cycle sustainability. 
- Ensure that new materials are commercially available for the advanced reactor fleet.

- Deploy advanced reactors rapidly into the marketplace.

\subsection{Regulatory Requirements}

Regulation of existing nuclear power plants as well as advanced nuclear reactors and fuel facilities must meet high expectations for public safety. The high level of confidence the public aurrently has in the ability of the NRC to protect general health and safety must be maintained. Regulatory agencies must begin addressing the challenges involved in developing a regulatory framework for new reactors and fuel recylling technologies. The study participants identified the following goals:

- Establish a significantly more efficient, consistent regulatory process.

- Establish a collaborative industry/ regulatory agency rel ationship to address non-LWR technologies (i.e., advanced reactors and fuel facilities). Such a relationship will optimize the licensing and design function, while maintaining regulatory objectivity and independence.

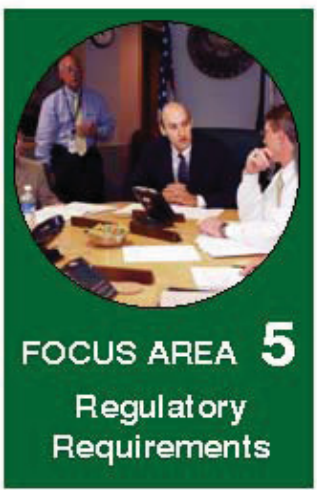

- Develop a standardized, automated application process for all technologies.

- Ensure harmonization of safety standards to facilitate safe global deployment of new technologies.

\subsection{Safeguards and Security}

The need to maintain aurrent high levels of safeguards and security (S\&S) as well as develop new technologies for safeguards is a pervasive, core requirement for the nuclear energy industry. Currently, maintaining S\&S involves labor-intensive and expensive approaches that rely on guns, guards, and gates. Implementation of S\&S must ensure future cost competitiveness. The analysis participants identified several goals:

- Optimally use technology to ensure materials accountability and effectively assess and manage physical and cyber threats.

- Implement "safeguards-by-design" philosophies in the design of reactors and the fuel cycle.

- Develop and implement international S\&S agreements to minimize proliferation.

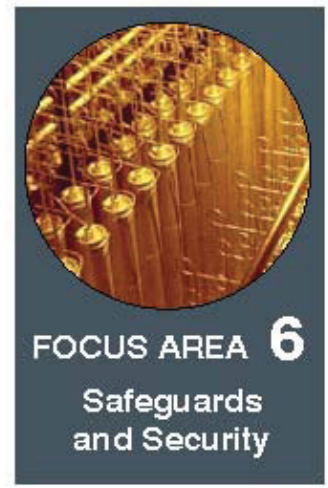

These three general areas go hand-in-hand in forming an integrated and effective system that is optimized for both S\&S effectiveness as well as incremental cost efficiency. As such, the following technologies will be required for materials accountability and physical and cyber threats:

- Online and at-line advanced detection instrumentation (radiation and non-radiation based),

- Advanced data integration and control systems, and

- Containment and surveillance, tags and seals, and intrusion detection and neutralization.

Formalized implementation of "safeguards-by-design" philosophies must take into consideration both domestic licensing requirements as well as international S\&S agreements. In other words, the "safeguards-by-design" concept must go beyond an individual facility design and be applied in aggregate for the nuclear fuel cycle. 


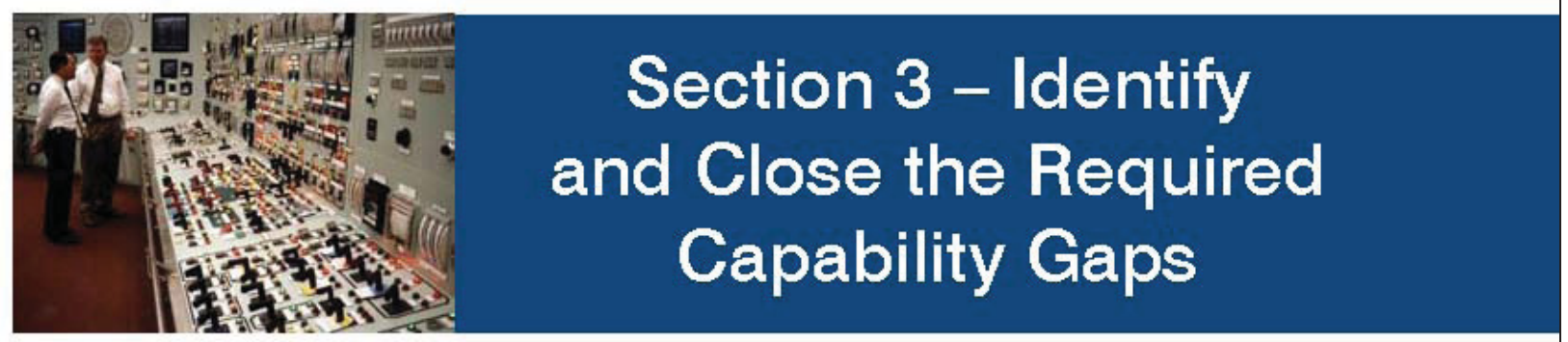

To achieve the nuclear energy industry goals artioulated in Section 2, certain capabilities must be available in the 2010 to 2050 time frame. This section identifies the required capabilities and defines any gaps that exist between currently available capabilities and those required in the future. Further, a mechanism for closing each identified gap is proposed.

As disaussed in Section 2, diverse nuclear energy industry groups provided input into defining the goals for the 2010 to 2050 time frame in the form of workshops, focus group meetings, and interviews. Appendix $A$ describes the process used to identify the necessary capabilities to achieve industry goals. In Appendix B, the identified capabilities are mapped to priority capabilities identified in workshops held in Columbus, Ohio, and Washington, D.C. Table 3-1 presents the required priority capabilities for each of the six focus areas. These capability requirements formed the basis for a follow-on gap analysis conducted by workshop participants to define necessary actions to meet nudear energy industry goals for the 2010 to 2050 time frame.

\subsection{Process to Identify and Close Capability Gaps}

Figure 3-1 illustrates the multistep process to identify the capability gaps and proposes mechanisms to close the gaps. Each required capability was evaluated to determine the following key characteristics or properties:

1. Whether the capability is unavailable today and is required by a number of other industries. If so, the closure strategy is to transfer and adopt the technology to the needs of the nuclear energy industry. Examples include advanced instrumentation and control, high-performance computing, nano-materials, general-use digital sensors, and knowled ge capture techniques.

2. Whether the capability is unavailable today and is of interest to other industries as well. If so, the dosure strategy is to co-develop the capability with the other industries. Once developed, the capability must be validated and verified (V\&V) for use in nuclear applications and deemed to be acceptable by the NRC, if it is safetyrisk significant. Examples indude heavy section welding, specialized sensors, nondestructive evaluation techniques, operational training, and advanced maintenance technologies for heavy process equipment.

3. Whether the required capability is unique to the nuclear industry and exists today only in the United States. If so, the closure strategy is to ensure that the capability 
Table 3-1. Required Priority Capabilities for 2010 to 2050

\begin{tabular}{|c|c|}
\hline Focus Area & Required Priority Capabilities \\
\hline $\begin{array}{c}\text { Existing LWRs } \\
\text { and ALWRs }\end{array}$ & $\begin{array}{l}\text { - SSC Reliability. Manage the reliability of the plant SSC materials through the plant's } \\
\text { extended lifetime } \\
\text { - Fuel Performance. Improve fuel performance } \\
\text { - Technology Innovations. Adopt technology innovations to enhance plant performance } \\
\text { and workforce productivity } \\
\text { - Manufacturing and Construction. Enhance manufacturing and construction methods } \\
\text { for plant life extension upgrades and construction of new plants }\end{array}$ \\
\hline $\begin{array}{c}\text { Workforce } \\
\text { Development }\end{array}$ & $\begin{array}{l}\text { - Optimize Training. Optimize training through adoption of proven approaches from } \\
\text { other industries, greater use of technical training centers, new methods, and improved } \\
\text { skill and aptitude assessment tools } \\
\text { - Knowledge Management. Adopt knowledge management methods and techniques to } \\
\text { enhance cross-generational knowledge retention, workforce development, and effective } \\
\text { use of lessons learned } \\
\text { - Sustainable R\&D. Enhance nuclear education/training and research infrastructure to } \\
\text { deliver a more effective multiyear, sustainable science and engineering R\&D program to } \\
\text { train the next generation of scientists and engineers } \\
\text { - Innovative Energy Educator. Enhance innovative energy educator programs to } \\
\text { effectively reach K-12 students - working with industry to build the pipeline }\end{array}$ \\
\hline $\begin{array}{l}\text { Sustainable } \\
\text { Fuel Cycle }\end{array}$ & $\begin{array}{l}\text { - Geologic Repository. Develop a geologic repository for the disposal of used nuclear } \\
\text { fuel and HLW } \\
\text { - Interim Storage. Develop an interim storage facility for receipt of used nuclear fuel } \\
\text { - Recycling Technologies. Develop recycling technologies that are economically } \\
\text { competitive, increasingly proliferation resistant, and minimize impact on waste disposal }\end{array}$ \\
\hline $\begin{array}{c}\text { Next-Generation } \\
\text { Reactors* }\end{array}$ & $\begin{array}{l}\text { - Fuels Development. Develop new fuels } \\
\text { - Heat Transport. Understand heat transport for new applications } \\
\text { - Modeling and Simulation. Enhance modeling and simulation capabilities } \\
\text { - Materials Development. Develop improved materials }\end{array}$ \\
\hline $\begin{array}{c}\text { Regulatory } \\
\text { Requirements }\end{array}$ & $\begin{array}{l}\text { - Licensing Efficiency. Improve the NRC license application and review process } \\
\text { - Basis for NGR and SFC. Establish risk-informed regulatory basis for next-generation } \\
\text { reactors (NGRs) and sustainable fuel cycle (SFC) activities } \\
\text { - Staffing. Ensure appropriate regulator staffing and effective staff training to meet job } \\
\text { requirements including NGRs and SFC activities }\end{array}$ \\
\hline $\begin{array}{l}\text { Safeguards and } \\
\text { Security }\end{array}$ & $\begin{array}{l}\text { - Optimized Technology. Use technology to optimize the use of guns/guards/gates } \\
\text { - Cybersecurity. Enhance cybersecurity capabilities to ensure systems security and } \\
\text { plant safety } \\
\text { - "Safeguards-by-Design." Design advanced safeguards approaches and technology } \\
\text { into ALWRs, NGRs, and fuel facilities }\end{array}$ \\
\hline
\end{tabular}

* Includes grid-appropriate reactors, reactors for the production of process heat, and reactors required for closure of the fuel cycle. 
is maintained in an acceptable state of readiness and is accessible when needed. An example is the Advanced Test Reactor (ATR) at INL.

4. Whether the required capability is unique to the nuclear industry and is available globally. If so, the closure strategy is to ensure access and sustainability of the capability (through financial support, if necessary) and confirm the acceptability to the U.S. market (e.g., the NRC, owner operators, and the public). An example is the International Materials Aging Institute organized by Électricité de France.

5. Whether the nuclear-unique capability is unavailable globally. If so, then the U.S. nuclear energy industry, including the DOE, owner operators, designers, construction contractors, and relatcd organizations (such as EPR and INPO) must devclop and maintain the capability as long as it is required to meet the goals of the industry. Examples include advanced radiochemistry laboratories, process demonstration facilities, and fast test reactors.

The nuclear-unique, gap-closure mechanisms are highlighted in dark blue in Figure 3-1.

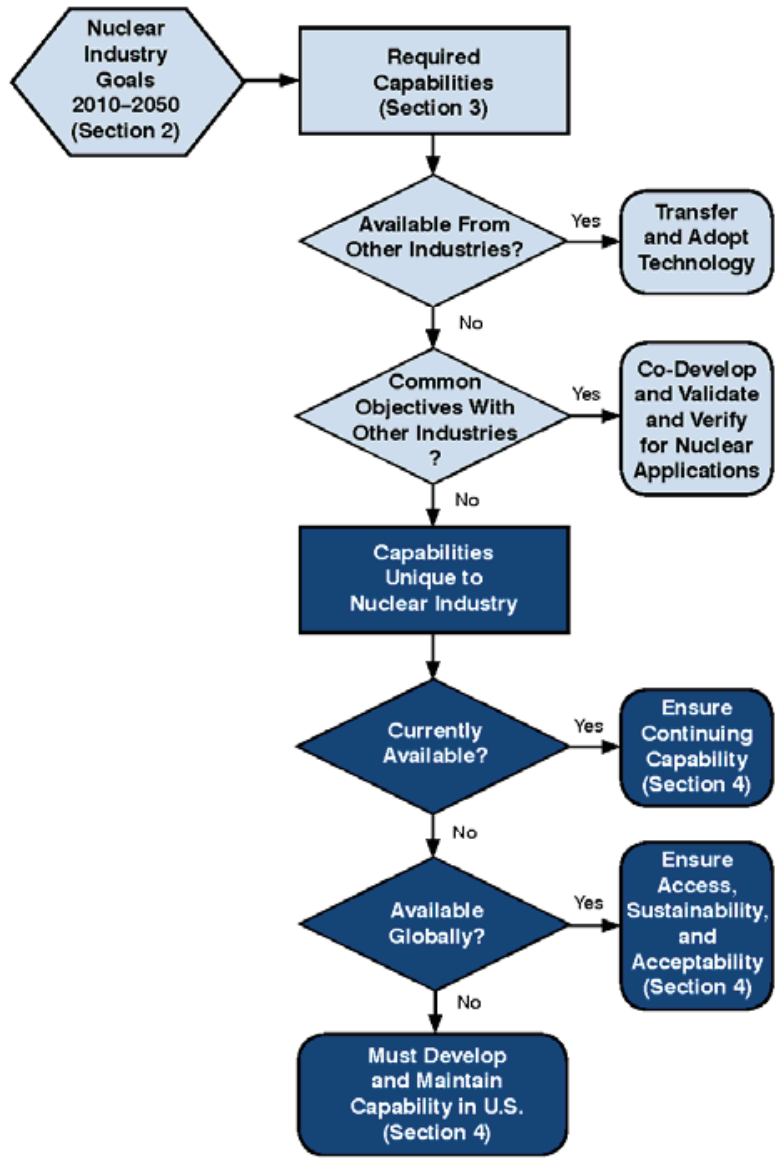

Figure 3-1. Capability Gap-Closure Flow Chart 


\subsection{Actions Needed to Close Required Capability Gaps}

The difference between the capabilities available today and those required in the future represents gaps that need to be filled. The process described in this section and Figure 3-1 provides guidance on effectively and efficiently closing these gaps.

An expert panel reviewed each required capability and determined which portion of the capability could be transferred from other industries and which portion could be effectively co-developed with other industries. The residual gaps in the required capabilities must be closed within the nuclear energy sector. Each residual gap was further apportioned (through a subjective process) to the following three capability categories: domestically available, globally available, and needs to be developed.

The process and results were reviewed by an independent set of outside experts from industry, academia, and the national laboratories, and were deemed to be appropriate. Each required capability and its gap closure strategy is summarized in Table 3-2. The expert panel determined that to receive a check mark on Table 3-2, the identified source needed to provide at least 25 percent of the needed capability.

For example, consider the actions required to close the capability gaps for NextGeneration Reactors - Materials Development from Table 3-2. Some of the materials technology can be jointly developed with the fossil power industry, particularly for the ultra super critical power plants that experience temperatures in excess of $700^{\circ} \mathrm{C}$. Portions of the materials development required for HTRs can be manufactured in existing U.S. facilities, such as thermal test reactors and hot cells. Some fast reactor materials development can be affected in global, nuclear-unique facilities, such as fast test reactors. Finally, some fast reactor materials demonstration can be conducted only in new facilities, such as a fast reactor demonstration project. 
Table 3-2. Sources of Required Capabilities to Meet Nuclear Energy Industry Goals in the 2010 to 2050 Time Frame

\begin{tabular}{|c|c|c|c|c|c|}
\hline Required Capability & $\begin{array}{l}\text { Transfer } \\
\text { From Other } \\
\text { Industries }\end{array}$ & $\begin{array}{l}\text { Co-Develop } \\
\text { With Other } \\
\text { Industries }\end{array}$ & $\begin{array}{c}\text { Nuclear } \\
\text { Unique - } \\
\text { U.S. Available }\end{array}$ & $\begin{array}{l}\text { Nuclear Unique - } \\
\text { Globally Available }\end{array}$ & $\begin{array}{c}\text { Nuclear Unique } \\
\text { - Develop } \\
\text { in U.S. }\end{array}$ \\
\hline & & & \multicolumn{3}{|c|}{ Nuclear Unique (see Section 4) } \\
\hline \multicolumn{6}{|l|}{ Existing LWRs and ALWRs } \\
\hline SSC Reliability & $\sqrt{ }$ & & $\sqrt{ }$ & $\sqrt{ }$ & \\
\hline Fuel Performance & & & $\sqrt{ }$ & $\sqrt{ }$ & \\
\hline Technology Innovations & $\sqrt{ }$ & $\sqrt{ }$ & $\sqrt{ }$ & & \\
\hline Manufacturing and Construction & $\sqrt{ }$ & $\sqrt{ }$ & & $\sqrt{ }$ & \\
\hline \multicolumn{6}{|l|}{ Workforce Development } \\
\hline Optimize Training & $\sqrt{ }$ & $\sqrt{ }$ & $\sqrt{ }$ & & \\
\hline Knowledge Management & $\sqrt{ }$ & & $\sqrt{ }$ & & \\
\hline Sustainable R\&D & $\sqrt{ }$ & & $\sqrt{ }$ & & $\sqrt{ }$ \\
\hline Innovative Energy Educator & $\sqrt{ }$ & $\sqrt{ }$ & & & \\
\hline \multicolumn{6}{|l|}{ Sustainable Fuel Cycle } \\
\hline Geologic Repository* & & & & & $\sqrt{ }$ \\
\hline Interim Storage $e^{\star \star}$ & & & & & $\sqrt{ }$ \\
\hline Recycling Technologies & & & $\sqrt{ }$ & $\sqrt{ }$ & $\sqrt{ }$ \\
\hline \multicolumn{6}{|l|}{ Next-Generation Reactors } \\
\hline Fuels Development & & & $\sqrt{ }$ & $\sqrt{ }$ & $\sqrt{ }$ \\
\hline Heat Transport & $\sqrt{ }$ & $\sqrt{ }$ & & & $\sqrt{ }$ \\
\hline Modeling and Simulation & $\sqrt{ }$ & & $\sqrt{ }$ & $\sqrt{ }$ & $\sqrt{ }$ \\
\hline Materials Development & & $\sqrt{ }$ & $\sqrt{ }$ & $\sqrt{ }$ & $\sqrt{ }$ \\
\hline \multicolumn{6}{|l|}{ Regulatory Requirements } \\
\hline Licensing Efficiency & $\sqrt{ }$ & & $\sqrt{ }$ & $\sqrt{ }$ & \\
\hline Basis for NGR and SFC & & & $\sqrt{ }$ & $\sqrt{ }$ & $\sqrt{ }$ \\
\hline Staffing & $\sqrt{ }$ & & $\sqrt{ }$ & & \\
\hline \multicolumn{6}{|l|}{ Safeguards and Security } \\
\hline Optimized Technology & $\sqrt{ }$ & $\sqrt{ }$ & & & \\
\hline Cybersecurity & $\sqrt{ }$ & $\sqrt{ }$ & & & \\
\hline "Safeguards-by-Design" & & & $\sqrt{ }$ & $\sqrt{ }$ & $\sqrt{ }$ \\
\hline
\end{tabular}

* Under the Nuclear Waste Policy Act of 1982, DOE's Office of Civilian Radioactive Waste Management is responsible for the development of the geologic repository. This capability is outside the scope of DOE NE and is not addressed in this report.

**Interim storage of used fuel prior to transport to the repository is the responsibility of the nuclear energy industry. This capability is outside the scope of DOE NE and is not addressed in this report. 


\section{Section 4 - Required Nuclear Energy R\&D Facilities}

The analytis provided in this section is focused on identifying the general types of nuclear facilities required to realize the needed unique nuclear capabilities previously identified in Section 3. In turn, these capabilities will enable the nuclear energy industry to achieve the 2010 to $2050 \mathrm{goals}$ described in Section 2. Facilities in the context of this section can span the range of plant simulators that provide dassroom training for power plant operators, to heavily shielded, hot cell facilities for examination and testing of irradiated materials. Additional examples of the nuclear-unique capabilities requiring facilities indude the ability to irradiate and test materials to evaluate the reliability of plant component materials, and to support development of recyling technologies that are economically viable, more resistant to proliferation, and improve waste disposal.

A fully coordinated complementary assessment entitled "Required Assets for a Nuclear Energy Applied Research and Development (R\&D) Program" is being led by INL. This assessment will identify various facilities now available throughout the $\mathrm{DOE}$ complex, in the domestic energy or related industries, or internationally that could be called upon to implement the R\&D capabilities described in this analysis. This analysis will provide input to that assessment. Outcomes and recommendations from that assessment are expected by the end of September 2008.

Finally, the facilities addressing these capabilities require significant capital investment and substantial annual resources to operate and maintain. Facilities aurrently available within the DOE complex, industry, and academic institutions will satisfy only a fraction of the requirements. In some cases, international collaboration could provide access to a portion of the needed facilities. However, aurrently available facilities fall short of providing several of the critical capabilities required to achieve the nuclear energy industry's long-range goals. In addition, given the age and original purpose of existing facilities, many DOE facilities require additional investment for improvements and needed modifications.

\subsection{Identification of Facility Requirements}

As mentioned previously, the nuclear-unique capabilities identified in Table $3-2$ require facilities in order to conduct necessary research. The general facility types identified in this analysis include nuclear education facilities, thermal irradiation facilities, fast irradiation facilities, radiochemistry laboratories, hot cells for separations, hot cells for post-irradiation examination (PIE), thermal transport, fuel development laboratories, licensing demonstration for HTRs, licensing demonstration for fast reactors, and special ized engineering devel opment labor atories (cold labor atories). Subject matter experts from academia, industry, and national laboratories identified these facility types 
during a round table discassion. Table 41 provides a summary mapping of capabilities into facilities. Specifically, the unique nudear capabilities listed in Table 3-2 are mapped with the type of nuclear facility (or facilities) required. In the context of Table 4l, facilities refer to all types of facilities-whether owned by industry, government, or available for use as a result of international collaborations.

As a specific example, the first entry in Table 3-2 is "SSC Reliability," which will require irradiation of materials followed by PIE of these test specimens to determine the effects of irradiation on the remaining useful life of the material. This capability maps into two types of facilities-thermal irradiation and hot cells for PIE and materials testing. The same logic was used to map the balance of the capabilities into the facilities as shown in Table 41 .

Brief descriptions of the required nuclear facilities follow in Section 4.2.

\subsection{Description of Resources}

The resources listed in Table 4-1 are briefly described below. INL staff are conducting a companion study to review existing domestic and international facilities for material condition, capability, readiness, and availability.

\subsubsection{Nuclear Education}

Nuclear education facilities provide hands-on education and training for the next generation of scientists and engineers. These facilities indude teaching laboratories, research laboratories, training centers, research and training reactors, reactor and fuel recycle training simulators, hot cells for research activities, and computation centers.

Enhancement of nudear education and training infrastructure enables creation of more effective multiyear $R \& D$ programs to train next-generation scientists and engineers. It also creates opportunities to leverage infrastructure investments through development of collaborative user facilities and research programs and helps provide additional internship and cooperative education opportunities for students as well as rotational assignments for nuclear energy industry workers. Increases in both undergraduate and graduate populations require commensurate growth in nuclear education facilities to ensure students are educated on modern and relevant techniques and instrumentation. Investment in nuclear education and training facilities encourages optimization of education and training programs through introduction of new methods, tools, and greater use of technical training centers. In addition, it provides an opportunity to more fully integrate knowled ge management methods and techniques into education and training to enhance cross-generational knowled ge retention, work force development, and effective use of lessons learned.

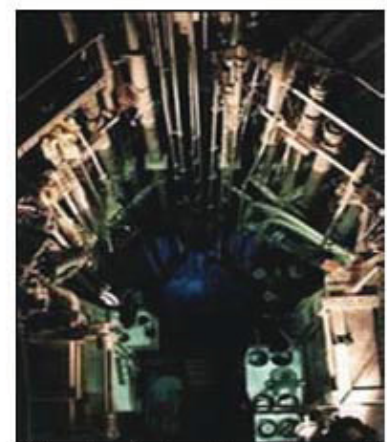

Using the University of Missouri Research Reactor Center's resources, students participate in the Nafonal Science Foundation's (NS's) Research Experience for Undergrad coes Program (REU). The in-depth hands-on research opportunities offered through REU whet the appeftes of college undergradiote students to pursue advanced degrees in nuclear science and engineering. 
Table 4-1. Matrix of Necessary Nuclear-Unique Capabilities and Required Resources

\begin{tabular}{|c|c|c|c|c|c|c|}
\hline 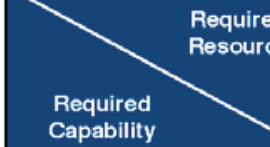 & $\begin{array}{l}\text { Nuclear } \\
\text { Education } \\
\text { Facilities }\end{array}$ & $\begin{array}{l}\text { Thermal } \\
\text { Irradiation } \\
\text { Facilities }\end{array}$ & $\begin{array}{c}\text { Fast } \\
\text { Irradiation } \\
\text { Facilities }\end{array}$ & $\begin{array}{c}\text { Radio- } \\
\text { Chemistry } \\
\text { Laboratories }\end{array}$ & $\begin{array}{l}\text { Hot Cells for } \\
\text { Separations }\end{array}$ & $\begin{array}{l}\text { Hot Cells for } \\
\text { PIE }\end{array}$ \\
\hline \multicolumn{7}{|l|}{ Existing LWRs and ALWRs } \\
\hline SSC Reliability & & $\mathbf{x}$ & & & & $\mathbf{x}$ \\
\hline Fuel Performance & & $\mathrm{x}$ & & $\mathbf{x}$ & & $\mathbf{x}$ \\
\hline Technology Innovations & $\mathbf{x}$ & & & & & \\
\hline \multicolumn{7}{|c|}{ Manufacturing and Construction } \\
\hline \multicolumn{7}{|c|}{ Workforce Development } \\
\hline Optimize Training & $x$ & & & & & \\
\hline Knowledge Management & $x$ & & & & & \\
\hline Sustainable R\&D & $x$ & & & & & \\
\hline \multicolumn{7}{|l|}{ Sustainable Fuel Cycle } \\
\hline Recycling Technologies & $\mathbf{x}$ & $\mathbf{x}$ & $\mathbf{x}$ & $\mathbf{x}$ & $\mathbf{x}$ & $\mathbf{x}$ \\
\hline \multicolumn{7}{|l|}{ Next-Generation Reactors } \\
\hline Fuels Development & $\mathbf{x}$ & $\mathrm{x}$ & $\mathrm{x}$ & $\mathbf{x}$ & & $\mathrm{x}$ \\
\hline Heat Transport & $x$ & & & & & \\
\hline Modeling and Simulation & $\mathbf{x}$ & $\mathbf{x}$ & $\mathbf{x}$ & & & \\
\hline Materials Development & $x$ & $\mathrm{x}$ & $x$ & $x$ & & $x$ \\
\hline \multicolumn{7}{|l|}{ Regulatory Requirements } \\
\hline Licensing Efficiency & $\mathbf{x}$ & & & & & \\
\hline Basis for NGR and SFC & $x$ & & & & & \\
\hline Staffing & $x$ & & & & & \\
\hline \multicolumn{7}{|l|}{ Safeguards and Security } \\
\hline "Safeguards-by-Design" & $\mathbf{x}$ & & & $\mathbf{x}$ & $\mathbf{x}$ & \\
\hline
\end{tabular}

Increasing research opportunities will increase enrollment in graduate degree programs in universities across the United States. NEI and several operating nuclear utilities and nuclear vendors have initiated educational outreach activities to encourage interest in science and technology careers. The nuclear energy industry and government also have been proactive in undertaking several initiatives (such as EPRI's in-service inspector training and qualification program) to improve training of workers and increase knowledge retention as the current workforce ages and retires.

Although several leading universities across the United States have nuclear education facilities, growth has not kept pace with the recent increase in demand or the number of students pursuing degrees in nuclear science and engineering. Further evaluation to define the specific nuclear education facilities is required. To augment available Federal funding sources (DOE, NRC, and NSF), the use of public-private partnerships could be investigated to provide funding for upgrading current facilities and constructing new facilities. Training of nuclear workers is generally tailored to individual plants and 


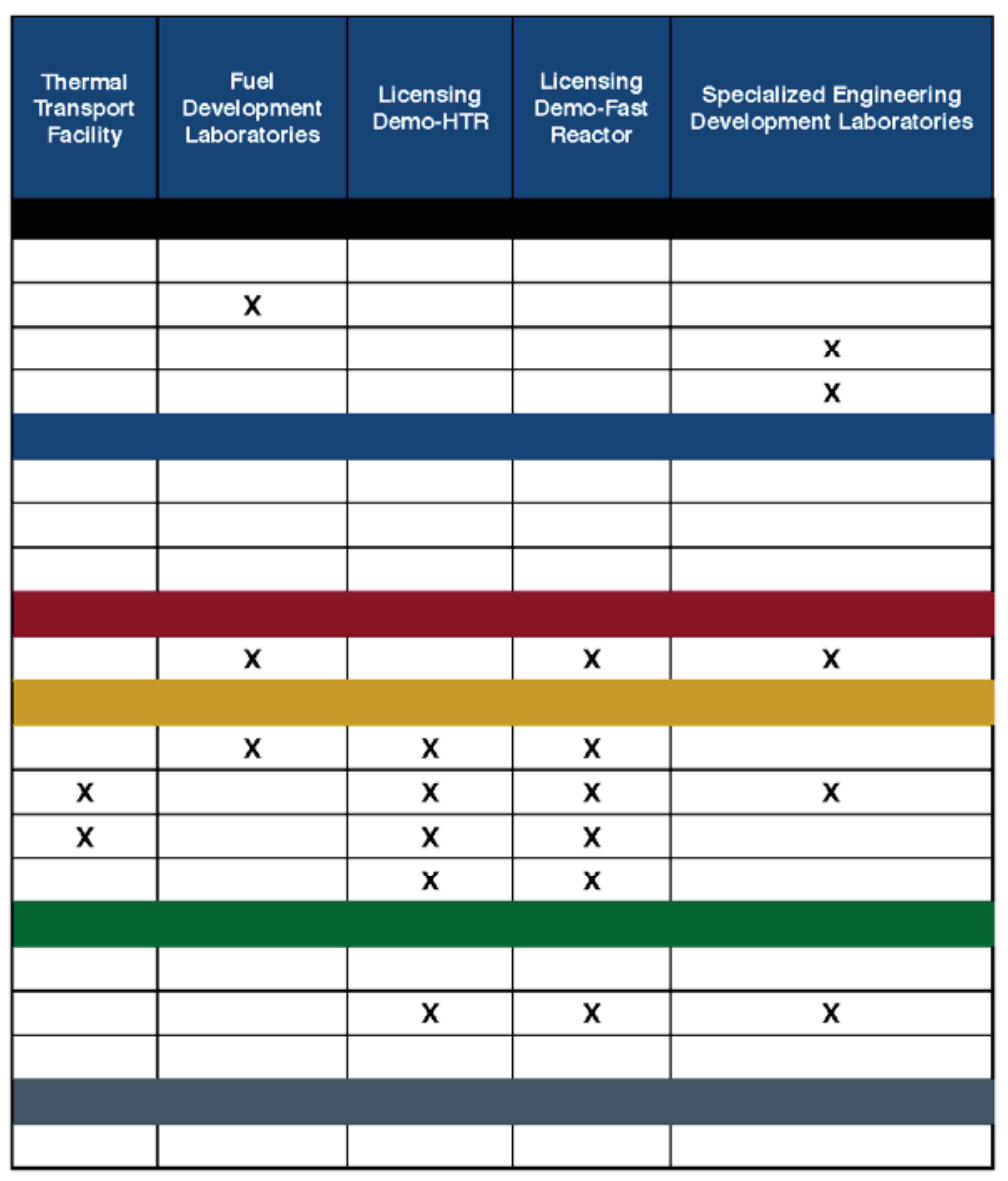

conducted at the plant site. More recently, INPO has undertaken an initiative to provide standardized and centralized training. The feasibility of regional training centers and additional standardized training programs should also be evaluated.

\subsubsection{Thermal Irradiation}

To manage the aging of reactor vessel material and core internals and develop higher burnup LWR fuels, the nuclear energy industry must have access to test reactors that can irradiate materials and fuels in neutron spectra that match those of the operating LWRs and the soon-to-be-built ALWRs. Only with neutron flux levels that exceed those in service can realistic accelerated aging results be achieved. In addition to the irradiation capability, the PIE and testing of materials and fuels require hot cells of sufficient size and capability to provide meaningful knowledge of the aging mechanisms (see Section 4.2.6). 
These capabilities are necessary to support the extended life (greater than 60 years) operation of the existing 104 U.S. reactors and to support the reliable and safe operation of the new fleet of ALWRs to be constructed in the next decade or so. The first commercial power orders in 30 years were placed in early 2008 by the Southern Company, NRG Energy, Inc, and South Carolina Electric and Gas Company. The results of the systematic irradiation and testing of new fuel designs will help reduce the time to develop reliable fuel designs that provide extended burnup of fuel (up to 100 GWd/MTU), resulting in improved economics and reduced

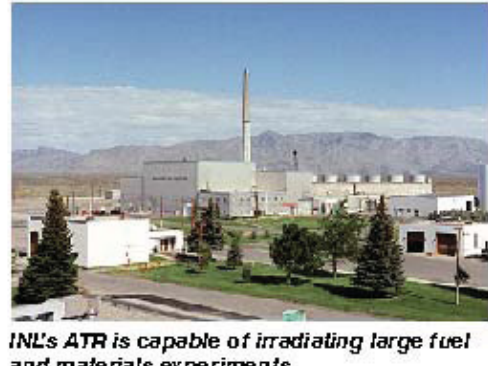
waste volume and shipments. Similarly, the ability to irradiate and test core materials and reactor vessel materials of sufficient size to reflect actual component behavior facilitates an optimal aging management strategy. Such a strategy includes the appropriate level of in-service inspection and informed run/repair/replace decisions, while maintaining the requisite levels of safety for these key components.

The nuclear energy industry requires thermal test reactor(s) of sufficient size and availability to irradiate new fuel design pins and material test specimens to provide prototypical results. The hot cells should be closely associated with the test reactors to minimize the radioactive materials transport issues and expenses (see Section 4.2.6).

Several such facilities are operating in the DOE complex. These include the ATR at INL, shown here, and the High Flux Isotope Reactor (HFIR) at Oak Ridge National Laboratory (ORNL). The ATR has recently been designated by DOE NE as a National Scientific User Facility for use by industry and academia, as well as by the traditional Naval Reactors and DOE users.

\subsubsection{Fast Irradiation}

To develop new fast reactors, significantly more test irradiation capabilities need to be provided for the research community. These capabilities are needed in the near-, mid-, and long-term. Currently, the United States uses irradiation capabilities in foreign countries to conduct limited tests on materials and fuel. Additional fast irradiation facilities are needed in the near-, mid-, and long-term to provide a source of fast neutrons to study materials aging issues and develop models for aging phenomena in fast reactors. For fast irradiation, these facilities should provide high fast flux levels to accel erate aging studies. Fast irradiation facilities are also needed to test fuels appropriate for use in a fast reactor. In addition to the irradiation capability, the PIE of fuels and testing of materials require hot cells of sufficient size and capability to provide meaningful knowledge of the damage mechanisms and fuel performance.

Fast reactors operate in harder neutron spectra than thermal reactors. The power density of fast reactors is generally higher than thermal reactors. Fast reactors generally use a heat transfer fluid other than water (e.g., liquid sodium), which must be compatible with reactor materials. Identification and assessment of stress, corrosion, and aging issues in this fast reactor environment are necessary for development of future fast reactors. Access to fast irradiation capabilities is needed to develop guidelines for construction materials for

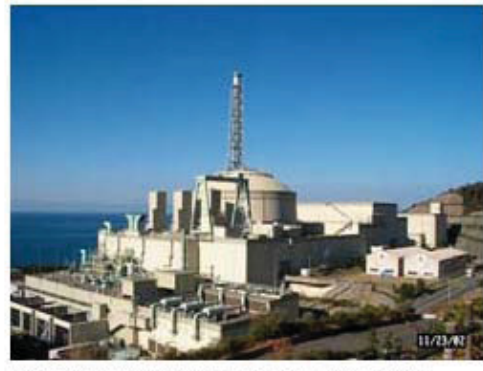

The Monju reactor in Japan represents one of severa' intemational fast irradiation facilities. 
fast reactors, develop appropriate fuel types for fast reactors, and eventually provide data to support licensing and construction.

Initially, small samples will be used to model the impact of irradiation damage. As the R\&D program progresses, the fuel testing will need to progress to larger and larger sample sizes, eventually reaching the lead test assembly (LTA) size to provide proof-ofprinciple testing. In the near-, mid-, and long-term, both materials irradiation and test fuel irradiation can take place in a research reactor environment where the sample sizes can be small, but the neutron fluences must be high. As fuel development activities get closer to commercial applications, larger facilities will be required to provide irradiation of full-length fuel pins as well as LTAs at prototypical conditions.

\subsubsection{Radiochemistry Laboratories}

Radiochemistry laboratories are needed throughout the near-, mid-, and long-term to support radiochemical, elemental, and isotopic analysis of nuclear fuels (un-irradiated and irradiated) and the resultant fission products, as well as materials and waste products encountered in nuclear fuel processing and fuel development. Detailed baseline analyses of feed materials and resultant products are critical to characterize how well separations processes perform during fuel recycle R\&D efforts. In addition to the separations needs, the new fuel for irradiation tests must be analyzed to ensure it meets requirements for irradiation and also analyzed post-irradiation to characterize the used fuel. Also, waste products will need to be well characterized to develop material balances and aging data needed for licensing.

Ideally, these radiochemistry laboratories would be located close to the hot cell or glove box facility performing the work. Close proximity to the R\&D facility will minimize radioactive/hazardous material transportation requirements and the time delays associated with those activities. Radiochemistry laboratories require licensing for an appropriate hazard categorization to adequately encompass the high levels of radioisotopes that will be present in the materials to be analyzed.

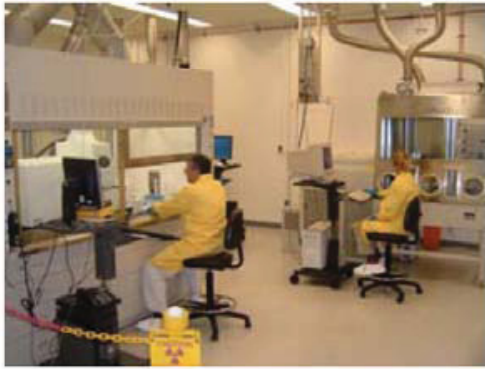

Radiochemistry faboratories provide chemical fume hoods and glove boxes for specialized analyses.

Radioactive specimens for analyses are generated prior to, during, and after R\&D tasks, requiring a wide variety of analytical measurements. A majority of the analytical techniques requires dissolution of the specimens into an aqueous matrix prior to analysis. These dissolutions are performed on representative sample portions in a chemical fume hood, glove box, or hot cell depending on the specific radioisotopes present and their activity levels. Specimens of irradiated fuels are likely to require hot cell containment. Dilutions of the digested homogenized solutions are performed, which allow for handling of the analytical aliquots in chemical fume hoods in which most of the instrument or measurement techniques are conducted. Some techniques are performed on small solid aliquots of the samples and require more rigorous instrument containment such as a glove box or hot cell. Potential sample measurement techniques include (1) alpha/beta/gamma spectroscopy, (2) plasma and thermal ionization mass spectroscopy, (3) gas chromatography, (4) ion chromatography, and (5) various wet chemical techniques such as titrations and spectrophotometric measurements. In some cases, such as fuels development, the radiochemistry lab must 
also be capable of measuring particle morphology, pellet density, and oxygen to metal ratios with very high accuracy, employing high-end microscopy, $\mathrm{X}$-ray diffraction, laser techniques, and high-temperature melts on the raw samples.

These labs will require the standard basic chemistry laboratory support systems such as bench tops, chemical fume hoods, deionized water soures, conditioned electrical power, and appropriate ventilation systems. Additional nuclear infrastructure requirements include radiological containment systems and protection support, liquid and solid radiological waste disposal systems, and staff trained to the rigorous stand ards needed to operate in a nuclear facility.

\subsubsection{Hot Cells for Separations}

About 96 percent of used nuclear fuel pellets are made up of uranium. About 3 percent of the fuel is highly radioactive fission products along with small amounts (less than 1 percent) of plutonim. Chemical separations are needed for the recovery of the unused uranium and the plutonium along with isolation of the fission products into an appropriate waste form. In addition, the nuclear energy industry needs data to support licensing efforts for new fuel recycling facilities capable of processing thousands of kilograms per year. Development of these new separations processes requires the use of real used fuel, which requires

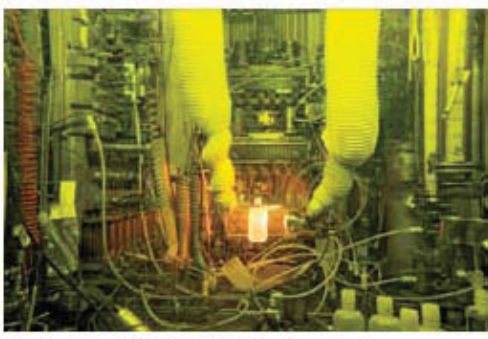

Ho t cells at ORNL's Radiochemical Development Center are among the DOE hot cell facilitifes that may be used to test fuel recycle separations. the availability of heavily shielded hot cell facilities.

Implementation of these new processes on a commercial scale will require testing first at a small scale (tens of kilograms) followed by scale-up. Small hot cells are needed in the near-, mid-, and long-term for small-scale separations R\&D programs. Larger hot cell facilities are need ed in the mid- to long-ter $m$ to provide engineering-scale demonstration of the most promising used fuel recycle technologies so those technologies can be deployed in a full-scale plant.

Industry and government agencies need to work collaboratively to improve the cost effectiveness of separations technologies that meet waste management and nonproliferation goals. Hot cell facilities are needed to conduct the research necessary to develop new separations processes to close the fuel cycle and provide data enabling scale-up of those processes. These facilities also generate important and unique experimental materials (e.g., recycle fuel and waste products) from used fuel, which are needed to ensure that composition, physical properties, and stability are well understood prior to committing resources for licensing and construction.

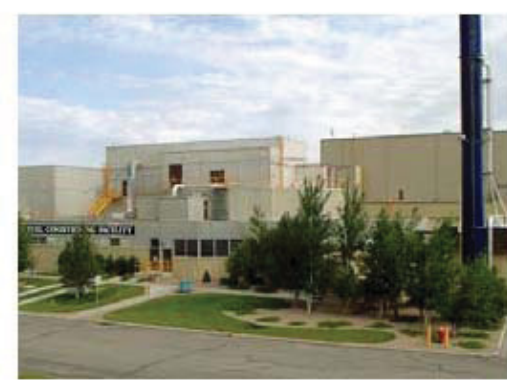

The Fuel Conditioning Facility at INL is used for testing separations technology

Hot cell facilities will provide a shielded environment for conducting experimental tasks in support of dosing the fuel cycle. The facilities will need ventilation systems to ensure containment of the radioactivity and support systems to move heavy shielded casks containing spent fuel into the hot cells. Operations inside the hot cells will be conducted remotely using robotic technologies. An infrastructure of waste management services, trained staff, appropriate $S \& S$ controls, as well as aurrent safety basis authorization will be necessary to support these hot cell facilities. 
As mentioned in the introduction to Section 4, many DOE sites have hot cell facilities that could be used for separations research work. These facilities span a wide range of size and activity type to support fuel recycle. Additionally, international facilities could provide limited support. As the efforts to close the fuel cycle expand, new hot cell facilities will be needed in the mid- to long-term to ensure that $\mathrm{R} \& \mathrm{D}$ facilities can provide design data and ongoing support for the new fuel recycle

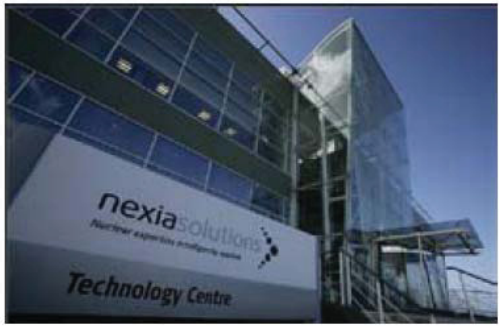

The British Technology Centre may be available for intermational collaborations. plant.

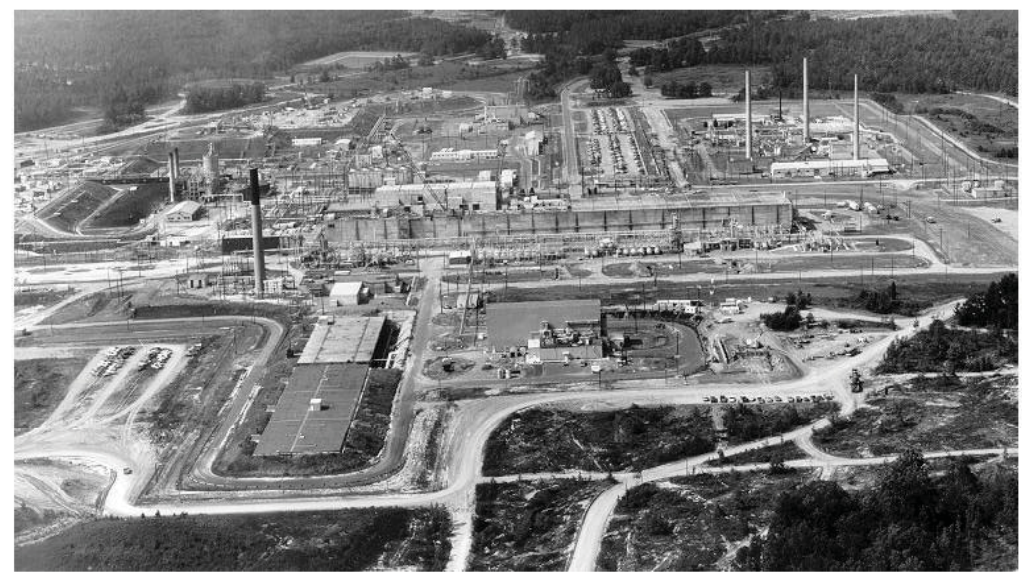

H Canyon at Savannah River Site is an example of a large-scale chemical separations facility.

\subsubsection{Hot Cells for PIE}

Hot cells for PIE and testing of fuels and irradiated materials are key facilities for understanding phenomena that limit fuel performance or useful life of reactor components. The cells must be of sufficient size to accommodate full-size fuel assemblies and have sufficient capability to provide meaningful examination and testing results. Ideally, they are situated close to the test reactor where the fuel/materials were irradiated or close to major transportation arteries to facilitate the shipment and handling of fuel/ materials casks from operating reactors.

The hot cells for PIE will be required for extended life operation of the current fleet of LWRs and soon-to-be-built ALWRs for qualification of fuel for the next generation of reactors, new materials development for higher-temperature reactor components, qualification of fuels developed in the sustainable fuel cycle program, etc.

The hot cells should have the capability to fully examine and analyze test specimens on macroscopic and microscopic scales. Required irradiated material testing capabilities for a wide range of temperatures and environments include strength, toughness, hardness, stress corrosion crack growth rates, environment-assisted fatigue crack growth rates, and related parameters. For fuel examination, additional tests are required, including fission 
gas analysis, fuel pellet strain and cracking, cladding strain, corrosion product analysis, neutron radiography, and related parameters. In addition, the hot cells should be able to test and verify nondestructive examination technologies for future use in the field for fuel and material examinations.

Several large hot cell facilities in the DOE complex can be used to examine and test irradiated fuels and material. They include the Hot Fuel Examination Facility (HFEF) at INL and the Irradiated Fuels Examination Laboratory and Irradiated Materials Examination and Testing Laboratory (IMET) at ORNL. The HFEF has recently been designated by DOE NE as part of the ATR National Scientific User Facility for use by industry and academia, as well as by the traditional Naval Reactors and DOE users.

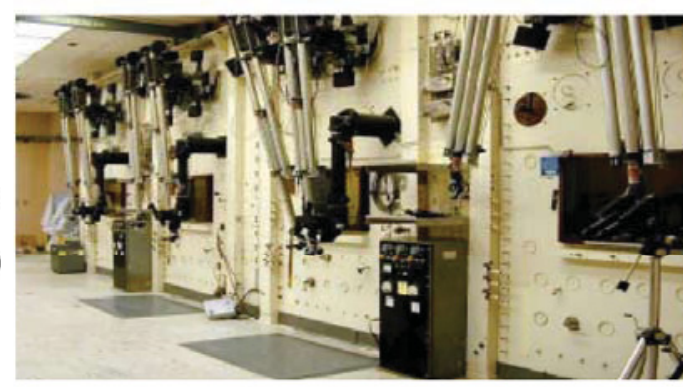

The Los Alamos Chemistry and Metallurgical Research Facility Wing 9 hot cells are used for irradiated fuel and materials examination, metallurgical examination, and neutron radiography.

\subsubsection{Thermal Transport}

Process industries such as oil refining and chemical manufacturing may be able to transfer many capabilities required for effective and efficient heat transfer and transport for nuclear energy heat sources. However, there are thermal transport considerations that are unique to the use of nuclear energy as a heat source. Most of these considerations relate to the reliability of the intermediate heat exchange (IHX) system that separates the nuclear system from the end user's systems. The effects of end-user facility (process heat users) upsets and transients on the safety and reliability of the high-temperature process heat reactor are also important.

Essentially, all applications of commercial nuclear energy have focused on the generation of electricity using the Rankin steam cycle. South Africa is developing a pebble bed HTR to produce electricity using the Brayton cycle with a gas turbine. Electrical grid disturbances and failures have been integrated in the design of existing LWRs. However, upsets and transients in refineries and chemical plants need to be considered in the design and operating procedures for process heat reactors. Large-scale loop tests will help the licensing of process heat reactors by providing credible benchmarks for system interactions analyses.

A thermal transport loop of sufficient size (about 60 megawatts thermal [MWth]) is required to demonstrate the reliability of the IHX design and materials for temperatures up to $950^{\circ} \mathrm{C}$. The results of the tests will be used to benchmark the thermal-hydraulics codes used in the design of process heat reactors for safety and reliability assessments. The same thermal loop may be used to evaluate the efficacy of large-scale water-spliting processes to make hydrogen and oxygen. Some bench-scale processes use either chemical assistance, high-temperature electrolysis, or a combination of the two to produce hydrogen from water. A fossil-fueled thermal loop would act as the heat source for the prototype testing. Eventually, the selected process would be evaluated with an HTR, either in the demonstration plant, Next-Generation Nuclear Plant (NGNP), or a followon facility. Such a facility may be used to test small high-temperature components for ultra super critical boilers in advanced, high-efficiency coal plant programs.

Currently, no such thermal loops operate in the DOE complex; industrial facilities of sufficient size are designed for lower temperatures. The thermal test facility would need to be a new facility designed and built for this purpose and operational by 2012 . 


\subsubsection{Fuel Development}

Research and testing programs are needed to increase the current LWR fuel burnup to the $100 \mathrm{GWd} / \mathrm{MTU}$ limit as envisioned by industry, requiring an increase in the current enrichment licensing limit. In addition, significant research and testing are needed to develop new fuel types for NGRs. Generally, these new reactors push the aurrent envelope of acceptable burnup and irradiation damage to the extent that one- of-akind experiments with several different combinations of fuel, cladding and irradiation conditions are needed to begin optimizing the fuel design. These new fuel types require extensive pre and post-irradiation examination.

Fuel development facilities are needed over the near-, mid-, and long-term for developing new fuel/clad types that will achieve high burnups, converting products from the used fuel separations processes into appropriate fuel forms-either cxide, carbide, or metal - and developing new fuels for NGRs. For TRISO fuels, specialized coaters are required to apply the pyrolytic carbon and silicon carbide coatings. These facilities contain glove boxes with appropriate ventilation to contain radioactive materials as well as capability to provide inert at mosphere, if needed. In some cases, these facilities might require significant security resources depending on the amount and type of material being processed.

Fuel development facilities also require analytical equipment in proximity to characterize the feed materials prior to fabrication as well as characterization of the final fuel form. Analyses can include dimensional verifications, chemical and isotopic analysis, measurement of the cxide to metal ratios, characterization of the feed particle morphology and other parameters.

Fuel development will proceed through a number of phases prior to devel opment of full-scale assemblies, induding fabrication of pellets or particles; assembly of pellets into rodlets, or particles into pebbles or compacts; irradiation of rodlets, pebbles, or compacts; scaleup to full pins; irradiation of pins; scaleup to full assemblies; and irradiation of LTAs. The phases of fuel devel opment will require larger and larger facilities to handle the increase in mass and attendant increase in security requirements. Some of the required capabilities for the next-generation reactor fuels are available in strategic global facilities.

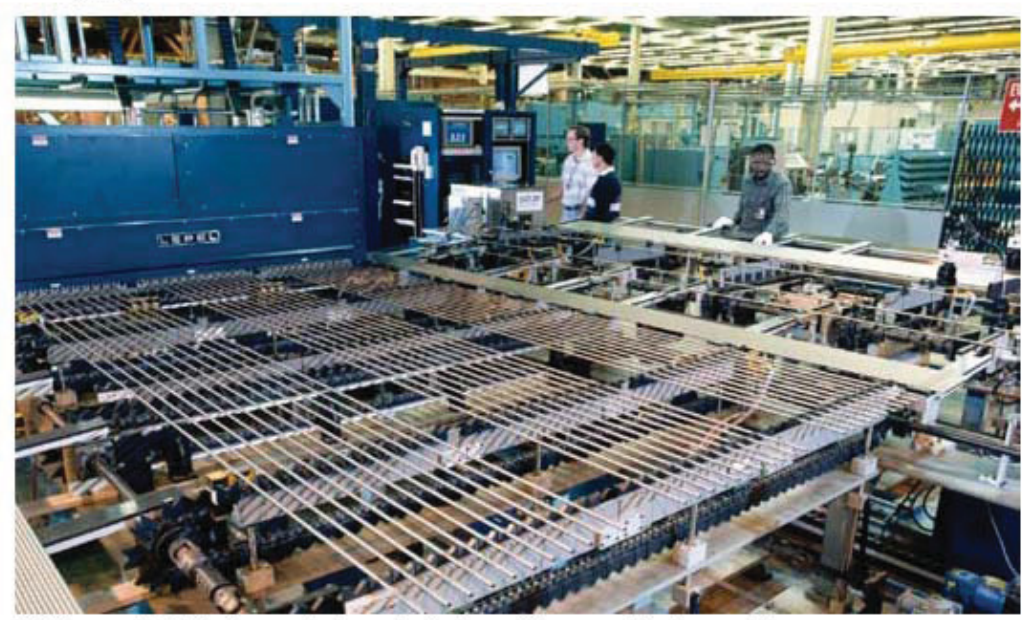

Unlike new LWR fuel, recycled reac tor fuel must be assembled remotely 


\subsubsection{Licensing Demonstration for an HTR}

ALWRs are excellent for generating electricity. However, if nuclear energy is to be used to provide other forms of energy, it will be necessary to develop and deploy highertemperature reactor systems. The use of water as a coolant restricts ALWRs to about $300^{\circ} \mathrm{C}$. Process heat applications for refiners and chemical plants require $250^{\circ} \mathrm{C}$ to $550^{\circ} \mathrm{C}$; oil shale and tar sands processing requires $300^{\circ} \mathrm{C}$ to $600^{\circ} \mathrm{C}$; and electricity and steam cogeneration requires $350^{\circ} \mathrm{C}$ to $800^{\circ} \mathrm{C}$. Reforming natural gas into hydrogen requires $500^{\circ} \mathrm{C}$ to $900^{\circ} \mathrm{C}$, and thermo-chemical and high-temperature electrolysis of water into hydrogen and oxygen requires $800^{\circ} \mathrm{C}$ to $1000^{\circ} \mathrm{C}$. To further reduce the U.S. industrial carbon footprint and our reliance on imported, conventional hydrocarbons, we must develop gas-cooled reactor systems.

High-temperature gas (hel ium) cooled reactors have been under devel opment for more than 40 years, and a prototype system using pebble bed fuel technology will be started up in South Africa in 2015. However, to be deployable in the United States, the technology must be demonstrated in a plant licensed by the NRC of sufficient size to convince end users of its viability and potential competitiveness with other forms of energy-particularly natural gas.

NGNP is a publidy/privately funded partnership to design, license, build, and operate a high-temperature nuclear demonstration plant designed to generate electricity and hydrogen using process heat to drive a thermo-chemical or high-temperature electrolysis water-splitting process. The plant will use fuel particles coated with layers of pyrolytic graphite and silicon-carbide to provide fuel-level containment of radioactive materials, helium as a coolant, and graphite as the moderator.

Across the globe, there are only a few small gas-cooled test reactors, including the HighTemperature Test Reactor (HTTR) in Japan and the 100-MWth reactor in China. The only large-scale HTR prototype is the Pebble Bed Modular Reactor (PBMR) in South Africa, which begins construction in 2010, designed to use the Brayton cycle to generate electricity. The NGNP would be the only large ( 300 to $400 \mathrm{MW} / \mathrm{th}$ ) demonstration of a combined electricity/process heat plant. An artist's concept of the NGNP is shown below.

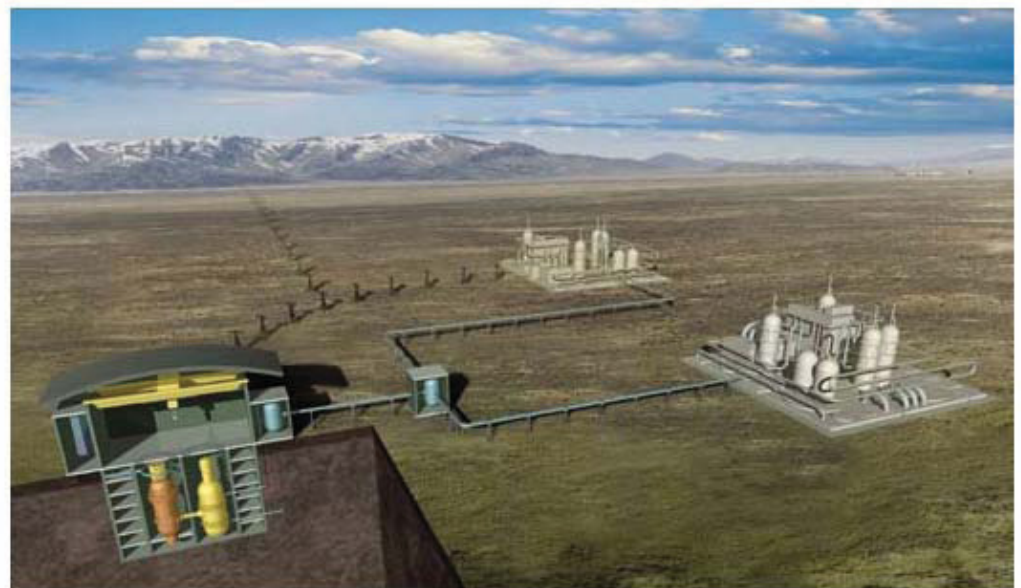

NGNP is a public-private parbership project to design, license, build, and operate an $H$ TR for the production of electricity and process heat.

Nuclear Energy for the Future

28 Required Rese arch and Developmert Capabilites - An holustry Perspective 


\subsubsection{Licensing Demonstration for a Fast Reactor}

Fast reactors are expected to play a central role in sustaining the nuclear fuel cycle in the 2030 to 2050 time frame. They will permit the transmutation of undesirable fission products into more "repository friendly" waste products with reduced toxicity. Fast reactors can also be designed to utilize certain fissionable materials as fuel, reducing highlevel waste volumes and extending repository life. Finally, fast reactors can be designed to transform non-fissile isotopes of uranium into useful nuclear fuel, greatly increasing the energy derived from uranium ore.

Fast reactors have been under development globally since the early 1950s. Most of the fast reactors have been sodium cooled. The extreme incompatibility of sodium and water has proven to be problematic for most of the demonstration, prototype, and test reactors. The deployment of fast reactors to support a sustainable fuel cycle requires the licensing of a demonstration fast reactor by the NRC.

The funding of a fast reactor demonstration plant is the responsibility of the Federal government. The private sector may own and operate future fast reactors when the fuel costs become competitive with an open fuel cycle approach. The demonstration fast reactor will also be a research facility to test new fuel designs on a large scale, as well as new heat extraction, instrumentation, and control and coolant circulation technologies.

Most of the large-scale (greater the $200 \mathrm{MW}$ th) fast reactors built globally have been shut down for a variety of reasons. The last large fast reactor shut down in the United States was the Fast Flux Test Facility (FFTF), which was built to test fast reactor fuel designs and primary circuit components.

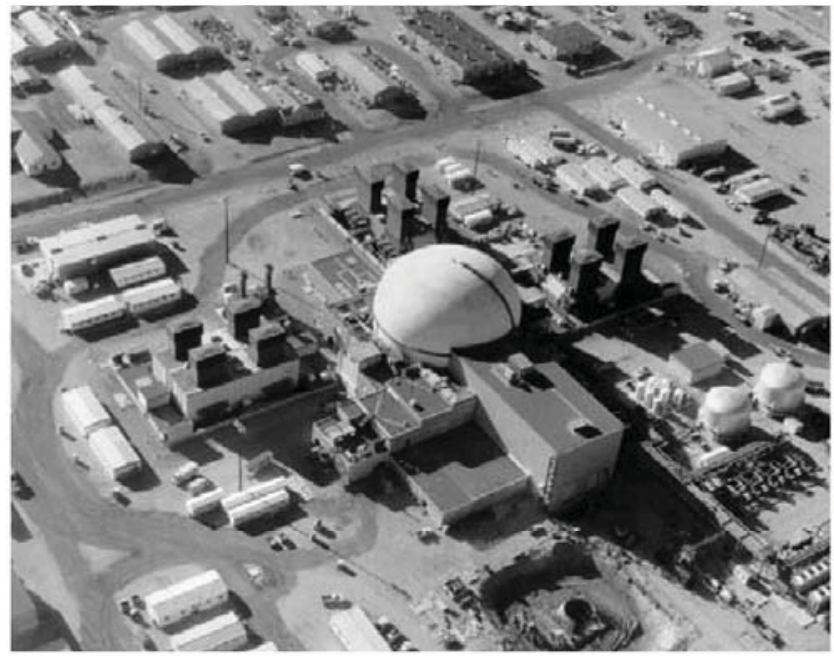

FFTF was the last large operating U.S. fast reactor. 


\subsubsection{Specialized Engineering Development Laboratories}

As the nuclear energy industry moves beyond the light water technology $\left(300^{\circ} \mathrm{C}\right)$, maximum hot leg temperatures, and an open fuel cycle, there will be a need for specialized engineering development facilities to provide engineering capabilities to support the goals of the industry in the 2010 to 2050 time frame. These facilities will deal with the nonradioactive testing and evaluation needs to ensure that the deployed power and process heat plants and fuel cycle facilities are safe and economically competitive.

These facilities must provide the special "unique to nuclear" capabilities that are not directly tied to fuel development and testing, fuel recycle chemistry, irradiated materials testing, and related areas. Historically, such capabilities have included seismic testing of major components, environmental qualification of equipment, flow loop testing, specialized sensor development, reactor simulator development, specialized welding process development and qualification, and specialized inspection and maintenance equipment development and qualification. Many facilities that currently provide these specialized capabilities are industrial, not DOE owned.

Many of the specialized engineering development facilities required for LWRs and ALWRs will still be required in the 2010 to 2050 time frame. Next-generation reactors and fuel recycling facilities will require additional capabilities, such as sodium flow loops for fast reactors, highly corrosive environment testing flow loops, instrumentation and equipment development qualification for sodium and high-temperature environments, in service inspection in sodium and high-temperature environments, and similar areas of interest. For some next-generation reactors, additional discussion with industry is warranted to define large component test requirements. 


\section{Section 5 - Priorities and Actions}

Action is required to provide the $\mathrm{R} \& \mathrm{D}$ capabilities to achieve the goals identified by the domestic nuclear energy industry for the 2010 to 2050 time frame. Although many of the required capabilities exist or are under development in other industries, several are unique to the nuclear energy industry and require specialized facility resources. These facility resources, identified in Section 4, require significant capital investment and substantial annual resources to operate and maintain. Facilities currently available in the DOE complex and internationally could satisfy many of the requirements. However, the available facilities fall short of providing many of the critical capabilities required to achieve the nuclear energy industry's long-range goals. In addition, given the age and original purpose of the existing facilities, many of the DOE facilities require additional investment to fund improvements and needed modifications.

Table 5-1 summarizes "unique-to-nuclear" R $\& \mathrm{D}$ facility priorities resulting from the nuclear energy industry goals for the 2010 to 2050 time frame and the required capabilities identified by participants in the Columbus, Ohio, and Washington, D.C., workshop and focus group.

Table 5-1. Summary of Nuclear R\&D Facility Priorities

\begin{tabular}{|c|l|l|l|l|}
\hline Prio rity & \multicolumn{1}{|c|}{ Focus Area } & \multicolumn{1}{c|}{ Facility } & \multicolumn{1}{c|}{ Purpose } & \multicolumn{1}{c|}{ Notes } \\
\hline \#1 (tie) & $\begin{array}{l}\text { Existing LWRs } \\
\text { and ALWRs }\end{array}$ & $\begin{array}{l}\text { Thermal irradiation } \\
\text { and PIE facilities }\end{array}$ & $\begin{array}{l}\text { Maximize benefit from } \\
\text { current reactor fleet }\end{array}$ & $\begin{array}{l}\text { Existing facilities provide } \\
\text { needed capabilities for } \\
\text { materials aging and fuels } \\
\text { improvement }\end{array}$ \\
\hline \#1 (tie) & $\begin{array}{l}\text { Workforce } \\
\text { Development }\end{array}$ & $\begin{array}{l}\text { Nuclear Education } \\
\text { facilities }\end{array}$ & Educate and train & $\begin{array}{l}\text { Further evaluation of } \\
\text { needs required }\end{array}$ \\
\hline \#2 (tie) & $\begin{array}{l}\text { Next-Generation } \\
\text { Reactors }\end{array}$ & $\begin{array}{l}\text { HTR Licensing } \\
\text { Demonstration }\end{array}$ & $\begin{array}{l}\text { Develop and demonstrate } \\
\text { new applications for } \\
\text { nuclear energy }\end{array}$ & $\begin{array}{l}\text { Engineering } \\
\text { development and } \\
\text { component test facility } \\
\text { required }\end{array}$ \\
\hline Fuel Cycle & $\begin{array}{l}\text { Fuel Cycle R\&D } \\
\text { facilities }\end{array}$ & $\begin{array}{l}\text { Develop new, licensable } \\
\text { fuel fabrication and } \\
\text { separations technologies } \\
\text { to improve fuel } \\
\text { performance, enhance } \\
\text { resource recovery, reduce } \\
\text { proliferation risk, minimize } \\
\text { waste, and improve } \\
\text { economics }\end{array}$ & $\begin{array}{l}\text { Available hot cell } \\
\text { facilities with continued } \\
\text { maintenance and } \\
\text { upgrades should provide } \\
\text { needed capabilities } \\
\text { through laboratory-scale } \\
\text { research }\end{array}$ \\
\hline \#3 & $\begin{array}{l}\text { Next-Generation } \\
\text { Reactors }\end{array}$ & $\begin{array}{l}\text { Develop and demonstrate } \\
\text { fast reactor technology to } \\
\text { improve safety and help } \\
\text { ensure sustainable fuel } \\
\text { supply }\end{array}$ & $\begin{array}{l}\text { Fast Reactor } \\
\text { Licensing } \\
\text { Demonstration } \\
\text { development and } \\
\text { component test facility } \\
\text { required }\end{array}$ \\
\hline
\end{tabular}


Three of the facilities would be new-the HTR Licensing Demonstration, the Fuel Cycle R\&D facilities, and the Fast Reactor Licensing Demonstration. Each requires support facilities to fulfill its mission. The mechanisms available to provide the needed facility resources are (1) public investment, (2) public-private partnerships with federal and state government and nuclear energy industry investment, and (3) U.S. government investment leveraged through international collaboration and investment. Recent initiatives underway in DOE NE to work more closely with industry and to expand international collaboration will provide vehicles for the development of and access to needed facility resources. Given the nature and purpose of the required facility resources, each mechanism requires government investment. Of the identified facility priorities, plans are already underway to establish a public-private partnership to provide the necessary funding for the NGNP - a licensing demonstration of an HTR.

The responsibility for the funding required for the facility priorities resides primarily with DOE NE. To achieve the required investment for the new facilities and the maintenance and enhancement of current facilities, it is recommended that the DOE NE establish the Strategic Nuclear Energy Capability Initiative to provide a significant increase in multiyear investments in capabilities that enable the domestic nuclear energy industry to achieve its 2010 to 2050 goals. Funds provided through the Initiative would be used to maintain and modify currently available facilities and provide new R\&D facilities and associated equipment. This Initiative would also provide the financial foundation for DOE NE participation in public-private partnerships and international collaborations to leverage U.S. taxpayer investment in required capabilities and facilities.

Recommendations for the success of the Strategic Nuclear Energy Capability Initiative include the following:

- Use an integrated, time-phased, and user-driven approach. The initial focus would be on workforce development, procurement of needed research equipment, improvement in current $\mathrm{R} \& \mathrm{D}$ facilities, and development of user-driven concepts for new R\&D facilities. Developing user-driven concepts provides the rationale for building the investment over time to enable construction of new user-driven $R \& D$ priority facilities.

- Provide multiyear investments in a manner that enables efficient development and commissioning of the needed facilities.

- Engage the nuclear energy industry, universities, and the national laboratories in the development and evaluation of user-driven concepts. Conduct integrated periodic reviews of the Initiative.

Input from industry, university, and national laboratory participants in this analysis provided the following additional recommendations for the DOE NE that are key to effective delivery of needed capabilities to the domestic nuclear energy industry:

- Increase the use of enterprise models that allow proper trade-off studies, integrating the economics of various future scenarios for development and deployment of different reactor types and fuel recycling technologies.

- Expand international collaborations to provide required capabilities and ready access to research facilities.

- Define a living process for facility consolidation, retirement of old facilities, and possible replacement with new, improved facilities when appropriate.

- Define a process to ensure maintenance of the "balance of plant"-roads, support buildings, etc., for critical facilities to ensure sustainability of capabilities. 


\section{Section 6 - Conclusions and Benefits}

\subsection{Conclusions}

Four key conclusions can be derived from this analysis:

- A robust, fully trained workforce must be available for the domestic nuclear energy industry to continue with its proven record of delivering baseload electricity in a safe, reliable, and cost-effective manner.

- The industry has established meaningful goals that enhance the nation's energy security. These goals include extending the service life of the currently operating reactors, deploying ALWRs, developing a sustainable fuel cycle, and developing nextgeneration reactors leading to new applications for nuclear energy.

- Essential R\&D capabilities and facilities are required to enable the industry to achieve these goals. Through the establishment of a multiyear, user-driven Strategic Nuclear Energy Capability Initiative, the DOE NE can provide the needed capabilities and facilities.

- Successful establishment of the Initiative can also provide the DOE NE with the necessary foundation to build public-private partnerships and international collaborations to help provide the needed capabilities and facilities.

\subsection{Benefits}

Provision of the required capabilities and facilities will enable the nuclear energy industry to deliver the following benefits:

- Meet the ever-increasing demand for energy.

- Reduce carbon intensity of U.S. electricity production.

- Reduce reliance on imported hydrocarbons.

- Create a sustainable nuclear fuel cycle and reduce risk of proliferation.

- Leverage DOE investment to maximize return to the U.S. taxpayer.

We cannot rely on "silver bullets" to address either the national (or global) energy supplyf demand situation or to manage global climate change in the next 50 years. The only pragmatic solution to either of these critical issues is a robust portfolio approach with a balance of energy sources, including nuclear energy. This requires a long-term and prudent use of scarce resources. 


\section{Appendix A - Process to Identify Capability Requirements}

\section{A.1 Methods Used to Gather Input}

To meet the request of DOE NE's Assistant Secretary to “.... seek... the insight of universities, customers, suppliers, and competitors... through workshops, interviews, and other appropriate means...," Battelle employed a disciplined process to gather input through a variety of methodologies. These methodologies, listed in the following subsections, involved a cross-section of 40 experienced nuclear energy professionals within industry, academia, national laboratory, and regulatory sectors over a two-month time frame. The following sections provide specifics of each forum and participant engagement.

\section{A.1.1 Facilitated Workshop}

Battelle hosted a two-day facilitated workshop in Columbus, Ohio, on April 30 and May 1, 2008, with participation by 13 representatives from industry, academia, regulatory organizations, and national laboratories.

\section{A.1.2 Focus Group}

A one-day focus group was held in Washington, D.C., on May 6, 2008. Nuclear energy industry participants included 13 utility executives, technology vendors, suppliers, architect engineers, and construction contractors. Two national laboratory representatives from Argonne and Pacific Northwest also participated in the focus group.

\section{A.1.3 Individual Interviews}

A total of five 1-hour interviews (with six participants) were conducted, including noted leaders from both industry and academia.

\section{A.1.4 Web-Based Focus Group}

Inputs from eight nuclear science universities from across the nation were provided via a web-based focus group that was introduced at a National University Consortium/Idaho University Consortium Symposium held in Idaho Falls, Idaho, on May 21, 2008. Given project background information and a demonstration of the website tool, participants were asked to provide their input online within one week.

\section{A.2 Framework Used to Gather Input}

Battelle's approach was to provide a structure that elicited broad input initially, then continually synthesized the data stream. This was accomplished by using data gathered early in the process as a baseline for subsequent data gathering, while simultaneously managing for bias. 


\section{A.2.1 Facilitated Workshop}

Workshop participants identified the following six focus areas they considered to be critical to the future success of the nuclear energy industry: existing LWRs and ALWRs, workforce development, sustainable fuel cycle, next-generation reactors, regulatory requirements, and safeguards and security.

\section{A.2.1.1 Current State/Future State/Gap Identification}

For each of the six focus areas, participants outlined the current state and desired future state. Next, for each focus area, an R\&D capability "gap analysis" was performed.

Participants then identified the critical R\&D gaps that hinder/prevent realization of the desired future state.

\section{A.2.1.2 Prioritization Process}

Participants then sorted the identified R\&D capability gaps into near-term (2008-2015), mid-term (2016-2020), and long-term (2021-2030) time frame categories, based on the determined lead-time. They selected the top three R\&D capability priorities for each time frame (near-, mid-, and long-term).

\section{A.2.2 Focus Group}

Participants used the same basic framework that was developed for the facilitated workshop (as discussed in Section A.2.1). However, in addition to employing a facilitator, Battelle used computer-assisted facilitation with GroupSystems Meeting Room software. Each participant had access to a computer, linked with other computers in the room. Information entered by the recorder or other participants appeared simultaneously on all participants' computers. Each of the six previously identified focus areas (identified by the Ohio workshop participants) was validated by the focus group participants.

\section{A.2.2.1 Current State/Future State/Gap Identification}

For each of the six focus areas, participants reviewed the current state descriptions identified in the facilitated workshop held in Ohio. The facilitator led participants through a verbal process of adding, merging, or removing current state descriptions. The participants followed this same process to refine the future state descriptions.

To refine the "gap analysis," conducted in the Ohio workshop while managing for bias, the $R \& D$ capability gaps were presented as an entire list (without being organized by time frame). Using the computers and a "round robin" process, participants added items to the capabilities list. Participants entered the most important idea they considered relevant to the subject. After all participants entered one idea, the facilitator reviewed the new list, clarified the entries, and merged similar ideas. The participants then entered another idea, not already on the list, and the facilitator repeated the process of clarifying and merging the newly entered ideas.

\section{A.2.2.2 Prioritization Process}

The participants sorted the list of capabilities into near-term (2008-2015), mid-term (2016-2020), and long-term (2021-2030) time frame categories. Once this was accomplished (via computer), the participants prioritized the capabilities in each time frame by placing them (electronically) in priority order. To effectively manage for bias, the prioritization ranking of capabilities resulting from the earlier Ohio workshop was not provided to the focus group participants. The focus group results of the electronic ranking process were provided to participants in "real-time" via the GroupSystems software. 


\section{A.2.3 Individual Interviews}

Interviewees were asked a total of nine questions regarding current/future state of the nuclear industry, R\&D capability gaps, and capability prioritization. As such, responses to the questions integrated seamlessly with the workshop and focus group input. The interview questions are shown in Attachment A-1.

\section{A.2.4 Web-Based Focus Group}

To provide the broadest opportunity for participation, Battelle organized and conducted a web-based focus group. It elicited more than 30 comments and recommendations from university participants across the nation. Each participant was provided with a link and password to the site. Participants were able to see others' comments and respond to them as well as add new input. The website included the six focus areas and the topranked R\&D capability gap priorities for each focus area. Participants responded to each focus area $\mathrm{R} \& \mathrm{D}$ capability gap priority by answering the following question: What role should universities play in the closure of the gap and what benefits will be realized by university participation?

\section{A.3 Outcomes}

By using a sequential data refinement process involving a broad cross-section of industry, regulatory, and academic participants, recurring themes were clearly identified and three key outcomes achieved:

- Validation of the six focus areas originally identified by the Ohio workshop participants

- Substantiation and enhancement of identified R\&D capability gaps

- Independent verification of capability prioritization.

As such, confidence in the results is high, providing DOE NE with a meaningful benchmark of capabilities required to support the domestic nuclear energy industry that has been defined with substantial industry and academic participation. 


\section{Appendix A - Attachment A-1 NUCLEAR ENERGY R\&D CAPABILITIES OUTLOOK INTERVIEW QUESTIONS}

1) What terms/phrases come to mind as you consider the current state of the nuclear energy industry? What challenges/opportunities/threats exist (including "non-nuclear" barriers to adding nuclear capacity - for example, transmission capability)?

2) How do you envision the nuclear energy industry in the 2030-2050 time frame (future state)?

a) What capabilities are in the early, mid, and mature stage?

b) What key technology accomplishments have been achieved?

c) How do you see market demand growing (including traditionally nonnuclear areas)?

d) What other factors have key impact on shaping the industry in the future?

At a minimum, please consider the following in your response:

- Operating life extensions

- Construction of new operating plants

- Use of nuclear reactors for the production of process heat and hydrogen

- Development of advanced reactors, and

- Closing the fuel cycle

3) What technical challenges does the industry currently face in achieving the desired future state (2030-2050)? (Gap Analysis)

4) What regulatory challenges does the industry currently face in achieving the desired future state? (Gap Analysis)

5) What R\&D capabilities and supporting disciplines/processes are needed to enable industry to address these technical and regulatory challenges and achieve the desired future state? (Gap Analysis)
a) Near-Term (2008-2014)
b) Mid-Term (2015-2021)
c) Long-Term (2022-2028)

Prioritize the top two capabilities for each phase (near-, mid-, and long-term).

6) What $R \& D$ facilities are needed to enable industry to address these challenges and achieve the desired future state? (Gap Analysis)

7) Are there "lessons learned" from other industries that we could take advantage of in the nuclear industry? If so, what?

8) Please make any other comments or suggestions you feel would assist in identifying the R\&D capabilities needed by the U.S. nuclear energy industry over the next $20+$ years.

9) Would you recommend that we speak with any other colleagues to gather perspectives for this project? 


\section{Appendix B - Workshop/Focus Group Summaries}

\section{Workshop Summary/Focus Group Prioritization}

Tables B-1 through B-6 indicate the needed R\&D capability priorities identified in each focus area, broken down by time frame (near-, mid-, and long-term) as determined by the Battelle-hosted Ohio workshop participants (April 30 and May 1, 2008) and the

Washington, D.C., focus group participants (May 6, 2008).

From each focus area capability list, approximately three or four high-level, thematic priorities emerged (as shown at the top of each table). Each individual R\&D capability has been "mapped" to the high-level capability priorities, as shown in the "Priority Mapping" column of each table.

Input gathered via individual interviews and the web-based focus group was consistent with the priority themes identified in Tables B-1 through B-6. 
Appendix Table B-1. Light Water Reactors/Advanced Light Water Reactors Focus Area-Capability Priorities

1. Understand the reliability of the plant's systems, structures, and component materials through the plant's extended lifetime

2. Improve fuel performance

3. Adopt technology innovations to enhance plant performance and the productivity of the workforce

4. Enhance manufacturing and construction methods for plant life extension upgrades and construction of new plants

\begin{tabular}{|c|c|c|c|c|}
\hline \multirow[t]{2}{*}{ Capability Need } & \multicolumn{4}{|c|}{$\begin{array}{l}\text { Priority } \\
\text { Mapping }\end{array}$} \\
\hline & 1 & 2 & 3 & 4 \\
\hline \multicolumn{5}{|l|}{ Near-Term (2008-2015) } \\
\hline Extend life of irradiated material (or determine better ways to perform accelerated testing) & $\mathrm{x}$ & & & \\
\hline Create supply chain: Real-time logistics model & & & & $\mathrm{X}$ \\
\hline $\begin{array}{l}\text { Construction: Need modular build, shop build, zero re-work, innovative ways of demonstrating compliance, } \\
\text { standardized (form-fit-function to use multiple suppliers), certified plant and component designs }\end{array}$ & & & & $\mathrm{X}$ \\
\hline Replace obsolete instrumentation and control (I\&C) parts & & & $\mathrm{X}$ & \\
\hline Conduct research on fuel failures & & $\mathrm{X}$ & & \\
\hline Improve design, manufacturing, and construction methods & & & & $\mathrm{x}$ \\
\hline $\begin{array}{l}\text { Enhance fuel fabrication capabilities (higher density fuels and fuel burnup, and reduce the number of leakers- } \\
\text { "zero by } 10^{\circ} \text { ) }\end{array}$ & & $\mathrm{X}$ & & \\
\hline Perform modeling with experimental verification for specific systems & $x$ & & $x$ & \\
\hline Enhance modeling/monitoring to understand risk & $\mathrm{X}$ & & $\mathrm{x}$ & \\
\hline Improve pool-side examination of fuel assemblies & & $\mathrm{x}$ & & \\
\hline License and implement ALWRs & & $\mathrm{x}$ & $\mathrm{x}$ & $\mathrm{x}$ \\
\hline Develop advanced fuel forms with high Uranium-235 density & & $\mathrm{X}$ & & \\
\hline Develop failure-free fuel for operating reactors & & $\mathrm{X}$ & & \\
\hline \multicolumn{5}{|l|}{ Mid-Term (2016-2020) } \\
\hline Conduct fuel testing in reactors and licensing & & $\mathrm{X}$ & & \\
\hline Develop digital I\&C design concepts so that systems can change as technology advances & & & $\mathrm{x}$ & \\
\hline Develop a shared facility for development, deployment, and training & & & $\mathrm{x}$ & \\
\hline Complete replacement of I\&C during a single (economical) planning window & & & $\mathrm{X}$ & \\
\hline Update safety evaluation criteria to account for digital I\&C systems & & & $\mathrm{X}$ & \\
\hline Develop advanced full core simulation models to improve fuel performance predictability & & $\mathrm{x}$ & & \\
\hline $\begin{array}{l}\text { Develop additional improved inspection technologies which include in-situ monitoring, in-situ microchemistry, } \\
\text { stress, electrochemical noise }\end{array}$ & & & $\mathrm{X}$ & \\
\hline Create accurate predictive modeling of materials behavior & $\mathrm{X}$ & & & \\
\hline Develop the ability to inspect, repair, and replace at power & & & $\mathrm{X}$ & \\
\hline Conduct process simplification and increase automation & & & $\mathrm{x}$ & \\
\hline Employ remote NAC monitoring & & & $\mathrm{x}$ & \\
\hline Develop high heat flux test facilities & $\mathrm{X}$ & & & \\
\hline Implement modeling simulation/digital control systems which communicate with models & & & $\mathrm{X}$ & \\
\hline Establish the regulatory basis for extending burnup limits & & $\mathrm{x}$ & & \\
\hline Develop new disposition options for low and intermediate level waste & & & $\mathrm{X}$ & \\
\hline \multicolumn{5}{|l|}{ Long-Term (2021-2030) } \\
\hline Benchmark testing to validate models & $\mathrm{X}$ & & & \\
\hline Design materials for end-use & $\mathrm{X}$ & & & \\
\hline Develop transient test facility & & $\mathrm{X}$ & & \\
\hline Create new methodologies to accelerate testing of materials and plant components & $\mathrm{X}$ & & & \\
\hline Provide input into prognostic models & & & $\mathrm{x}$ & \\
\hline Employ corrosion-free materials & $\mathrm{X}$ & & & \\
\hline Improve hybrid, dry cooling sources, reduce manufacturing costs & & & & $\mathrm{X}$ \\
\hline Develop technologies to make use of rejected heat & & & & $\mathrm{x}$ \\
\hline Improve and/or implement new materials & $\mathrm{X}$ & & & \\
\hline $\begin{array}{l}\text { Utilize/adapt repair/refurbish technologies used by other industries such as military, aerospace, oil and gas, } \\
\text { shipbuilding industries, etc. }\end{array}$ & & & & $\mathrm{x}$ \\
\hline Realize $100 \%$ reliable, real-time inspection & & & $\mathrm{x}$ & \\
\hline Execute a digital control system which communicates with models & & & $\mathrm{X}$ & \\
\hline Improve water chemistry (i.e., nano-fluids) & $\mathrm{X}$ & & & \\
\hline $\begin{array}{l}\text { Develop/commercialize technologies for ALWR fuels and cladding materials (e.g., silicon carbide cladding, fertile- } \\
\text { free cores) }\end{array}$ & & $\mathrm{X}$ & & \\
\hline
\end{tabular}




\section{Appendix Table B-2. Workforce Development Focus Area-Capability Priorities}

1. Optimize training through adoption of proven approaches from other industries and greater use of technical training centers, new methods, and improved skill and aptitude assessment tools

2. Adopt knowledge management methods and techniques to enhance cross-generational knowledge retention, workforce development, and effective use of lessons learned

3. Enhance nuclear education/training and research infrastructure to deliver a more effective multiyear, sustainable science and engineering R\&D program to train the next generation of scientists and engineers

4. Enhance innovative energy educator programs to reach $\mathrm{K}-12$ students - working with industry to build the pipeline

\begin{tabular}{|c|c|c|c|c|}
\hline \multirow[t]{2}{*}{ Capability Need } & \multicolumn{4}{|c|}{$\begin{array}{l}\text { Priority } \\
\text { Mapping }\end{array}$} \\
\hline & 1 & 2 & 3 & 4 \\
\hline \multicolumn{5}{|l|}{ Near-Term (2008-2015) } \\
\hline $\begin{array}{l}\text { Fund a multiyear, sustainable nuclear science and engineering R\&D program to train the next } \\
\text { generation of scientists and engineers (DOE) }\end{array}$ & & & $\mathrm{x}$ & \\
\hline Develop a national certification program for skilled nuclear craft with attractive pay structure & $\mathrm{x}$ & & & \\
\hline $\begin{array}{l}\text { Implement a DOE/industry sponsored training program focused on personnel with high-school } \\
\text { education (education of people displaced from offshoring) }\end{array}$ & $\mathrm{x}$ & & & \\
\hline $\begin{array}{l}\text { Establish/continue innovative energy educator programs to reach students early }(K-12) \text { - working } \\
\text { with industry, etc., through a new Educator Forum to build the pipeline (DOE) }\end{array}$ & & & & $\mathrm{x}$ \\
\hline $\begin{array}{l}\text { Enhance DOE/industry-sponsored research funding for universities to support advanced degree } \\
\text { programs (researchers needed for national laboratories and Ph.D.'s for new NE faculty) }\end{array}$ & & & $\mathrm{x}$ & \\
\hline $\begin{array}{l}\text { Promote the message (to universities) that reactor plant design is multidiscipline-we need more } \\
\text { than just nuclear engineers (Industry) }\end{array}$ & & & $x$ & \\
\hline Implement effective mentorship programs & & $x$ & & \\
\hline Set up programs/practices/technologies to retain knowledge & & $x$ & & \\
\hline $\begin{array}{l}\text { Institute joint NRC/INPO/industry effort to optimize training toward competencies (performance- } \\
\text { based) }\end{array}$ & $x$ & & & \\
\hline Accelerate industry/NRC acceptance of virtual simulator technology (control room or laptop) & $x$ & & & \\
\hline $\begin{array}{l}\text { Benchmark with military, airlines, process industries (i.e., simulator training, distance education } \\
\text { training) }\end{array}$ & $x$ & & & \\
\hline $\begin{array}{l}\text { Consider skilled crafts in plant design-making their job easier and ultimately decreasing the } \\
\text { number of staff required }\end{array}$ & $x$ & & & \\
\hline \multicolumn{5}{|l|}{ Mid-Term (2016-2020) } \\
\hline $\begin{array}{l}\text { Support development of a regional training reactor concept-operated jointly by a regional university } \\
\text { consortium, national laboratories, and industry (DOE) }\end{array}$ & $\mathrm{x}$ & & $\mathrm{x}$ & \\
\hline $\begin{array}{l}\text { Provide universities with needed major equipment to facilitate availability of radiation sources, such } \\
\text { as access to accelerators or enhancement of research reactors }\end{array}$ & & & $x$ & \\
\hline Automate equipment (e.g., automated start-ups and shutdowns) & & & $x$ & \\
\hline Use virtual technology (e.g., avatars) to share knowledge & $x$ & $x$ & & \\
\hline Develop automated processes to optimize the human element & & & $x$ & \\
\hline
\end{tabular}


Appendix Table B-3. Sustainable Fuel Cycle Focus Area-Capability Priorities

1. Develop a geologic repository for the disposal of used nuclear fuel and HLW

2. Develop an interim storage facility for receipt of used nuclear fuel

3. Develop recycling technologies that are economically competitive and more proliferation resistant and minimize impact to waste disposal

\begin{tabular}{|c|c|c|c|}
\hline \multirow[t]{2}{*}{ Capability Need } & \multicolumn{3}{|c|}{$\begin{array}{l}\text { Priority } \\
\text { Mapping }\end{array}$} \\
\hline & 1 & 2 & 3 \\
\hline \multicolumn{4}{|l|}{ Near-Term (2008-2015) } \\
\hline $\begin{array}{l}\text { Develop fuel cycle closure research, development, and demonstration facility for advanced fuel cycle } \\
\text { technologies and fuel qualification }\end{array}$ & & & $\mathrm{x}$ \\
\hline $\begin{array}{l}\text { Develop recycling technologies that are economically competitive and proliferation resistant and } \\
\text { minimize impact to waste disposal }\end{array}$ & & & $\mathrm{x}$ \\
\hline Develop and operate interim storage facility & & $\mathrm{X}$ & \\
\hline Begin building a sodium reactor & & & $\mathrm{x}$ \\
\hline Develop cogent NE facility/program deployment strategy & $\mathrm{X}$ & & \\
\hline Create improved uranium resource assessment and mining capabilities & & & $\mathrm{X}$ \\
\hline Build a pyro used nuclear fuel separations unit & & & $\mathrm{x}$ \\
\hline Learn from INL/ANL processing of experimental Breeder Reactor-II fuel for direct application to LWR fuel & & & $\mathrm{x}$ \\
\hline Identify alternate system approaches for the fuel cycle & & & $\mathrm{x}$ \\
\hline Develop/maintain fast reactor expertise (vendors, DOE, NRC) & $\mathrm{X}$ & & \\
\hline Identify appropriate waste forms & & & $\mathrm{x}$ \\
\hline Upgrade computer codes for modeling advanced design fuel behavior and benchmark to test results & & & $\mathrm{x}$ \\
\hline $\begin{array}{l}\text { Identify best facility for manufacturing and testing of the advanced recycling reactor lead test } \\
\text { assemblies }\end{array}$ & & & $\mathrm{X}$ \\
\hline Build a transient LWR test reactor to extend fuel burnup & & & $\mathrm{x}$ \\
\hline $\begin{array}{l}\text { Learn from others using MOX fuel (recycling staff and plant operators) from France, Japan, United } \\
\text { Kingdom, and Russia) }\end{array}$ & & & $\mathrm{X}$ \\
\hline Expand knowledge/expertise on the production and use of MOX & & & $\mathrm{X}$ \\
\hline $\begin{array}{l}\text { Study MOX Lead Fuel Assemblies from used nuclear fuel separated in another country in a few U.S. } \\
\text { reactors }\end{array}$ & & & $\mathrm{x}$ \\
\hline $\begin{array}{l}\text { Reexamine the costs and benefits of implementing a process that separates pure plutonium. Are there } \\
\text { any real reasons to believe we cannot safeguard it? }\end{array}$ & & & $\mathrm{x}$ \\
\hline \multicolumn{4}{|l|}{ Mid-Term (2016-2020) } \\
\hline Develop HLW repositories & $\mathrm{X}$ & & \\
\hline Develop appropriate HLW form (post-recycling) for long-term geologic disposition & & & $\mathrm{x}$ \\
\hline $\begin{array}{l}\text { Identify alternate recycling routes with appropriate safeguards, low waste generation, and fewer } \\
\text { process steps }\end{array}$ & & & $\mathrm{x}$ \\
\hline Develop recycling technologies for metal fuel use in a sodium fast reactor & & & $\mathrm{x}$ \\
\hline Initiate process development work on high potential processes & & & $\mathrm{x}$ \\
\hline \multicolumn{4}{|l|}{ Long-Term (2021-2030) } \\
\hline Expand/continue MOX program and Global Nuclear Energy Partnership (GNEP) program & & & $\mathrm{x}$ \\
\hline Develop extraction/recovery processes for all natural resources from recycling operations & & & $\mathrm{x}$ \\
\hline Develop HLW retrieval capability to begin closing fuel cycle & $\mathrm{X}$ & & \\
\hline Mine GNEP transmutation fuels outcomes for transferable knowledge & & & $\mathrm{x}$ \\
\hline Pilot and demonstrate work on high-potential processes & & & $\mathrm{x}$ \\
\hline Implement thorium fuel development program & & & $\mathrm{X}$ \\
\hline
\end{tabular}




\section{Appendix Table B-4. Next-Generation Reactors Focus Area-Capability Priorities}

Develop next-generation reactors including grid-appropriate reactors, reactors for the production of process heat, and reactors required for closure of the fuel cycle

1. Develop new fuels

2. Understand heat transport for new applications

3. Enhance modeling and simulation capabilities

4. Develop improved materials

\begin{tabular}{|c|c|c|c|c|}
\hline \multirow{2}{*}{ Capability Need } & \multicolumn{4}{|c|}{ Priority Mapping } \\
\hline & 1 & 2 & 3 & 4 \\
\hline \multicolumn{5}{|l|}{ Near-Term (2008-2015) } \\
\hline Initiate development and "proof of concept" for fast reactors & $\mathrm{x}$ & & $\mathrm{x}$ & $\mathrm{x}$ \\
\hline Identify possibilities for international collaboration using new or existing test facilities & $\mathrm{x}$ & $\mathrm{x}$ & $\mathrm{x}$ & $\mathrm{x}$ \\
\hline \multicolumn{5}{|l|}{ Develop decision model to determine right reactor mix } \\
\hline $\begin{array}{l}\text { Continue progress to simplify design, operation, and maintenance of future reactors to ensure safe } \\
\text { and efficient operation }\end{array}$ & $\mathrm{x}$ & $\mathrm{x}$ & $\mathrm{x}$ & $x$ \\
\hline Create component research and test facilities at universities and national laboratories & $\mathrm{x}$ & $\mathrm{x}$ & $\mathrm{x}$ & $\mathrm{X}$ \\
\hline Build a PRISM prototype for fuel testing & $\mathrm{x}$ & & & \\
\hline Develop concept of in-situ breeding/burning for high burnup fuel & $\mathrm{x}$ & & & \\
\hline \multicolumn{5}{|l|}{ Define roadmaps to achieve commercial applications } \\
\hline Continue NGNP program on current timeline & $\mathrm{x}$ & $\mathrm{x}$ & $\mathrm{x}$ & $\mathrm{x}$ \\
\hline $\begin{array}{l}\text { Develop fast reactor test capability (impacting factors: economics, sustainability/closing the fuel } \\
\text { cycle) }\end{array}$ & $x$ & & & \\
\hline Develop an alternative secondary coolant for LMR & & $\mathrm{x}$ & & \\
\hline Define materials characteristics & & & & $\mathrm{X}$ \\
\hline Develop an enterprise model to evaluate reactor use/grid systems/economics & & & $\mathrm{x}$ & \\
\hline $\begin{array}{l}\text { Co-develop modeling/simulation methods with regulators in the areas of neutronics, thermal- } \\
\text { hydraulic, and thermal-mechanical materials }\end{array}$ & & & $\mathrm{x}$ & \\
\hline Develop hydrogen production materials & & $\mathrm{x}$ & & $\mathrm{x}$ \\
\hline Demonstrate pilot-scale hydrogen-production process & & $\mathrm{x}$ & & \\
\hline $\begin{array}{l}\text { Evaluate near-term applications for hydrogen generated from chemically assisted water splitting in } \\
\text { support of current and future hydrogen economy }\end{array}$ & & $\mathrm{x}$ & & \\
\hline Increase testing/verification of hydrogen-production processes & & $\mathrm{x}$ & & \\
\hline \multicolumn{5}{|l|}{ Mid-Term (2016-2020) } \\
\hline Develop HTGR fuel manufacturing processes/capability & $\mathrm{x}$ & & & \\
\hline Develop high-temperature materials (code-qualified) for heat transfer systems & & & & $\mathrm{x}$ \\
\hline Develop instrumentation for a sodium-cooled fast reactor using actinide fuel & $\mathrm{x}$ & & $\mathrm{x}$ & $\mathrm{X}$ \\
\hline $\begin{array}{l}\text { Develop applications at intermediate temperature (i.e., } \sim 750^{\circ} \mathrm{F} \text { ) that displace premium fuels } \\
\text { (i.e., natural gas, other fossil-based fuels) for industrial applications such as chemical plants and } \\
\text { refineries) }\end{array}$ & & $\mathrm{x}$ & & \\
\hline Scale up for commercial production of hydrogen & & $\mathrm{x}$ & & \\
\hline Apply high-performance computing to development of new materials/fuel types & & & & $\mathrm{X}$ \\
\hline \multicolumn{5}{|l|}{ Long-Term (2021-2030) } \\
\hline Implement "materials-by-design" for advanced reactor concepts & & & & $\mathrm{X}$ \\
\hline Use process heat for oil shale development/exploitation & & $\mathrm{X}$ & & \\
\hline Use hydrogen and oxygen from nuclear heat to perform coal-to-liquids/coal-to-gas conversion & & $\mathrm{X}$ & & \\
\hline Successfully start up reactor selected by the NGNP program & $\mathrm{x}$ & $\mathrm{x}$ & & \\
\hline Develop domestic graphite manufacturing capability & & & & $\mathrm{x}$ \\
\hline Develop supercritical $\mathrm{CO}_{2}$ power cycle for LMR (and/or other high-temperature reactor) deployment & & & & $\mathrm{X}$ \\
\hline \multicolumn{5}{|l|}{ Develop Advanced Gen IV test reactor } \\
\hline $\begin{array}{l}\text { Enhance sensor development for use in new environments (high-fluence and high-temperature } \\
\text { conditions) }\end{array}$ & & & & $\mathrm{x}$ \\
\hline Conduct uranium oxycarbide (UCO) fuel proof of viability & $\mathrm{x}$ & & & \\
\hline Prepare to use commercially produced UCO and thorium-based fuels in high-temperature reactors & $\mathrm{x}$ & & & \\
\hline
\end{tabular}




\section{Appendix Table B-5. Regulatory Requirements Focus Area-Capability Priorities}

1. Improve the NRC license application and review process

2. Establish risk-informed regulatory basis for next-generation reactors and sustainable fuel cycle activities

3. Ensure appropriate regulator staffing and effective training of staff for job requirements, including nextgeneration reactors and sustainable fuel cycle activities

\begin{tabular}{|c|c|c|c|}
\hline \multirow[t]{2}{*}{ Capability Need } & \multicolumn{3}{|c|}{$\begin{array}{l}\text { Priority } \\
\text { Mapping }\end{array}$} \\
\hline & 1 & 2 & 3 \\
\hline \multicolumn{4}{|l|}{ Near-Torm (2008-2015) } \\
\hline Continue DOE support for new generation and completion of the Part 52 licensing process & & $\mathrm{x}$ & \\
\hline $\begin{array}{l}\text { Improve mechanism by which NRC manages organizational conflict of interest-making constrained } \\
\text { resources available to NRC and industry }\end{array}$ & & & $\mathrm{x}$ \\
\hline $\begin{array}{l}\text { Implement training programs for new NRC employees and provide adequate management oversight to } \\
\text { minimize variations in approach to regulatory requirements interpretation }\end{array}$ & $\mathrm{x}$ & & $\mathrm{x}$ \\
\hline $\begin{array}{l}\text { Develop collaborative design/regulatory relationship (NRC, International Atomic Energy Agency [IAEA], } \\
\text { Environmental Protection Agency [EPA], DOE, Army Corps of Engineers) }\end{array}$ & $\mathrm{X}$ & & $\mathrm{X}$ \\
\hline Develop framework for risk-based waste classification system & & $\mathrm{X}$ & \\
\hline Streamline licensing and accelerate lessons learned from international licensing experience & $\mathrm{X}$ & & \\
\hline Define regulatory requirements for licensing an integrated recycling reactor site & & $\mathrm{X}$ & \\
\hline $\begin{array}{l}\text { Develop and deploy regulatory courses regarding general design criteria (with DOE funding) through } \\
\text { collaboration between universities, industry, and NRC }\end{array}$ & & & $\mathrm{X}$ \\
\hline Enhance NRC's capability to evaluate a sodium fast reactor license submittal & & & $\mathrm{X}$ \\
\hline Process the Yucca Mountain license submittal in less than two years & $\mathrm{X}$ & & \\
\hline $\begin{array}{l}\text { Increase R\&D of analytical codes (advanced reactors), including thermal fluids, fuels, fission product } \\
\text { formation and transport, high-temperature materials, graphite }\end{array}$ & $\mathrm{x}$ & $\mathrm{x}$ & \\
\hline Employ a standardized, automated application process for ALWRs & $\mathrm{x}$ & & \\
\hline $\begin{array}{l}\text { Develop new, innovative ways for NRC to manage conflict of interest (independent review of capability) } \\
\text { to mirror industry-licensing capabilities in order to effectively validate }\end{array}$ & & & $\mathrm{x}$ \\
\hline \multicolumn{4}{|l|}{ Mid-Term (2016-2020) } \\
\hline Apply technology-neutral, risk-informed regulation & $\mathrm{x}$ & & \\
\hline $\begin{array}{l}\text { Enhance and expand NRC capabilities to handle next-generation recycling facilities (i.e., HTGR and } \\
\text { Liquid Metal) }\end{array}$ & & & $\mathrm{x}$ \\
\hline $\begin{array}{l}\text { Develop knowledge-based regulatory oversight system to assist in uniform application of regulations, } \\
\text { inspections, and audits }\end{array}$ & $\mathrm{X}$ & & \\
\hline Enhance and expand NRC capabilities (HTGR and LMRs in process currently) & & & $\mathrm{x}$ \\
\hline $\begin{array}{l}\text { Develop regulatory baseline for co-location of power reactor and process heat unit through DOE/national } \\
\text { laboratory }\end{array}$ & & $\mathrm{x}$ & \\
\hline Initiate an automated licensing process & $\mathrm{X}$ & & \\
\hline
\end{tabular}




\section{Appendix Table B-6. Safeguards and Security Focus Area-Capability Priorities}

1. Optimize the use of technology to minimize guns/guards/gates

2. Enhance cybersecurity capabilities to ensure security of plant systems and safety

3. Design advanced safeguards approaches and technology into ALWRs, next-generation reactors, and fuel facilities

\begin{tabular}{|c|c|c|c|}
\hline \multirow[t]{2}{*}{ Capability Need } & \multicolumn{3}{|c|}{$\begin{array}{l}\text { Priority } \\
\text { Mapping }\end{array}$} \\
\hline & 1 & 2 & 3 \\
\hline \multicolumn{4}{|l|}{ Near-Term (2008-2015) } \\
\hline $\begin{array}{l}\text { Implement risk-based, "safeguards-by-design" approach to fuel cycle facilities regulatory framework and } \\
\text { design }\end{array}$ & & & $\mathrm{X}$ \\
\hline $\begin{array}{l}\text { Develop the capability to examine trade-offs between security based on the use of intrusion prevention/ } \\
\text { protection and the use of more fault tolerant systems }\end{array}$ & $\mathrm{x}$ & & $\mathrm{x}$ \\
\hline $\begin{array}{l}\text { Ensure that Design Basis Threat (DBT) is firmly established and that nuclear facilities have deployed } \\
\text { appropriate measures }\end{array}$ & & & $\mathrm{X}$ \\
\hline Develop new S\&S technologies to meet new DBT and IAEA inspection requirements & $\mathrm{X}$ & & \\
\hline Develop a more frequent and effective personnel screening process & $\mathrm{X}$ & & \\
\hline Create S\&S framework for an interim storage facility & & & $\mathrm{X}$ \\
\hline Enhance cybersecurity development & & $\mathrm{X}$ & \\
\hline Strengthen international standards & & & $\mathrm{X}$ \\
\hline Develop S\&S framework for MOX transport and use & & & $\mathrm{X}$ \\
\hline Install state-of-the-art video camera monitoring and surveillance & $\mathrm{X}$ & & \\
\hline $\begin{array}{l}\text { Utilize detector development disciplines. Provide advanced security and material control and } \\
\text { accountability (MC\&A) detection systems }\end{array}$ & $\mathrm{x}$ & & \\
\hline R\&D to support long-term core & $\mathrm{x}$ & & \\
\hline \multicolumn{4}{|l|}{ Mid-Term (2016-2020) } \\
\hline Ensure fuel supply & & & $\mathrm{x}$ \\
\hline Apply "safeguards-by-design" technologies in new construction & & & $\mathrm{x}$ \\
\hline $\begin{array}{l}\text { Implement advanced, real-time S\&S data acquisition and advanced instrumentation technologies } \\
\text { (MC\&A) across the United States and worldwide }\end{array}$ & $\mathrm{x}$ & & \\
\hline Integrate S\&S concerns into fuel design & & & $\mathrm{x}$ \\
\hline Develop alternate aircraft defense technologies (other than mega-containment structures) & & & $\mathrm{X}$ \\
\hline Optimize real-time life cycle tracking of special nuclear material fuel (i.e., embedded tracking chips) & $\mathrm{X}$ & & \\
\hline Deploy national laboratory-developed "state-of-the-art" S\&S technologies to industry & $\mathrm{x}$ & & \\
\hline Encourage the use of weapons material (plutonium and uranium) for fast reactor start-up cores & & & $\mathrm{x}$ \\
\hline Realize long-life core & & & $x$ \\
\hline Expand use of automation/robotics & $\mathrm{x}$ & & \\
\hline
\end{tabular}




\section{Appendix C - Required Capabilities Derived From Other Industries}

Section 3 described a process for closing capability gaps to meet the goals of the nuclear energy industry in the 2010 to 2050 time frame. The gaps in capabilities that are unique to the nuclear energy industry are discussed in Section 4. The technologies transferred from or developed with other industries are discussed in this appendix. The discussions are organized in the five focus areas that rely on other industries (existing LWRs and ALWRs, workforce development, next-generation reactors, regulatory requirements, and safeguards and security). The capability description includes the applicability, the approximate percentage (to the nearest 25 percent) transferred or co-developed from other industries, and a brief description of the sources and capabilities. The sustainable fuel cycle focus area, which is unique to the nuclear energy industry, also is covered in Section 4 .

\section{C.1 Existing LWRs and ALWRs}

To keep the existing 104 LWRs and the fleet of the new ALWRs running safely and reliably requires additional capabilities. Following is a summary of the capabilities that may be derived from other industries. The nuclear energy industry has the lead responsibility to transfer or co-develop these capabilities with limited DOE involvement.

- Systems, Structures, and Components (SSC) Reliability (LWRs and ALWRs, about 25 percent derived) —SSCs that are common to fossil power plants and other process industries include refineries and chemical plants. These capabilities include in-service inspection, aging management strategies (deterministic and probabilistic), weld-repair techniques, sealing and gaskets, bolting and fasteners, operation and maintenance techniques, and related items. Thermal aging management strategies of nonmetallic components, such as high-voltage and control cables, and concrete will be developed in concert with other process industries and the suppliers.

- Technology Innovations (LWRs and ALWRs, about 75 percent derived)—A large share of the capabilities required to improve nuclear energy technologies will be transferred from other industries. These include fully automated control systems, advanced sensors, robotics, prognostic maintenance technologies, and related items. Additional capabilities will be co-developed with other high-pressure, process industries, such as for chemical and petrochemical plants. Similarly, advanced crew training technologies can be co-developed with other process industries as well as the airline industry.

- Manufacturing and Construction (ALWRs and major upgrades of LWRs, about 75 percent derived) - Advanced manufacturing and construction techniques exist in most capital-intensive industries. With some modifications, these capabilities can be transferred to the nuclear energy industry. Some examples of these capabilities include construction logistics and management; modular construction techniques; on-site manufacturing of heavy components, including local stress relieving of 
welds; heavy structure transport and lift; advanced concrete pours for reinforced, pre- and post-tensioned structures; automated welding (and inspection) of piping and structures; and related areas. Advanced heavy section welding and inspection techniques can be co-developed with other power and process industries.

\section{C.2 Workforce Development}

In maintaining a robust workforce for the 2010 to 2050 time frame, capabilities developed in or co-developed with other industries will prove invaluable to the nuclear energy industry. The issues faced in the nuclear energy industry are virtually identical to those of many other manufacturing and processing industries.

- Optimize Training (All areas, 75 percent derived) —Much of the work under way to optimize training in other industries (particularly the power, process, defense, and airline industries) will benefit the nuclear energy industry. Major efforts are under way to improve the effectiveness and efficiency of training for operators and maintenance personnel, designers and engineers, and welders and construction workers. These options include computer-based training, just-in-time and as-needed training, virtual reality-based training, and smart system-based training. In addition, major efforts exist to automate systems to eliminate the need for human supervision and control. Likewise, workplace assistive device developments will permit the more effective use of physically challenged individuals in work environments where obstructions previously existed —enlarging the workforce pipeline.

- Knowledge Management (All areas, 75 percent derived) — Of all knowledge, 85 percent is tacit, not formally documented in procedures and instructions. Most of the real insights into designing, manufacturing, operating and maintaining equipment and systems are locked in the memories of the experienced, but aging, workforce. All industries are faced with the "gray" (experienced) to "green" (new recruit) transition. Methods ranging from more traditional mentoring programs to sophisticated knowledge extraction techniques are under development to extract tacit knowledge, document it, and integrate it into training programs. The nuclear energy industry must transfer the more successful methods in the near-term to take advantage of the limited time remaining before the experienced workforce retires.

- Sustainable R\&D (All areas, 25 percent derived)—Some of the R\&D capability required in the nuclear energy industry is common to several other industries, drawing heavily from science and engineering disciplines (mechanical, electrical, control, chemical, industrial, engineering mechanics, computer science, and related areas). Therefore, the nuclear energy industry should take advantage of the efforts in DOE Office of Science, NSF, and other educational programs to provide the longterm R\&D necessary to fulfill those technical capabilities that not unique to the nuclear industry.

- Innovative Energy Educator (All areas, 100 percent derived) - The nuclear energy industry should support and rely on the national endeavors to attract students (K-12) into math, science, and technology studies. While the efforts are not well coordinated, they are all a piece of addressing the workforce challenge. Numerous strong student outreach programs exist through technical societies, industrial organizations, federal and state governments, colleges and universities. Focused efforts are needed to attract women into science and technology.

\section{C.3 Sustainable Fuel Cycle}

The sustainable fuel cycle focus area is unique to the nuclear energy industry and is covered in Section 4. 


\section{C.4 Next-Generation Reactors}

Heat Transport (Process heat reactors, 75 percent derived) — One of the key capabilities of the process heat reactors developed in the future is to transport heat efficiently and effectively with minimal concern for corrosion resulting from leakage, spillage, or intrusion into end-users' systems of the heat transfer medium. Most capabilities required for the nuclear energy industry in this vein derive from the process industries themselves. Heat is a required, but expensive, commodity in process industries and their commercial success is dependent upon effective transfer, transport, and use. Dry cooling condenser technologies developed in other industries should be adapted to the next-generation reactors to minimize water requirements. This will permit the incorporation of the expertise and experience gained in the process and power industries.

Modeling and Simulation (All areas, 25 percent derived) - With exception of capabilities that involve nuclear processes, modeling and simulation capabilities can be adapted from other areas of science and technology. High-performance computer and atomistic models are being developed by DOE Office of Science and others to promote basic understanding of physics, chemistry, and materials science. Complex system simulations are required to optimize design of process facilities such as refineries and chemical plants. The nuclear energy industry will take full advantage of the modeling and simulation capabilities for those processes and systems that are not unique to nuclear energy.

Materials Development (All areas, 25 percent derived) - The nuclear energy industry has many unique material aging issues, such as neutron irradiationinduced embrittlement, radiolysis of coolants in the core, and complex and corrosive environments in fuel recycling and separation systems. However, materials developed in other industries have equally challenging thermal and chemical conditions that can be adapted to nuclear applications after the proper V\&V of the material's "fitness for duty." The chemical and advanced fossil power industries have appropriate capabilities that could be applied in nuclear environments. The new ultra super critical coal plants have a target main steam temperature of $700^{\circ} \mathrm{C}$. Real opportunities exist to co-develop materials and manufacturing processes between the high-temperature reactor and ultra super critical industries.

\section{C.5 Regulatory Requirements}

Licensing Efficiency (All areas, 25 percent derived) - While most of the NRC licensing activities are unique to the nuclear energy industry, there are overlaps with other U.S. regulatory agencies, such as the EPA, Department of Transportation Pipeline Safety, and Food and Drug Administration. Additionally, every country in the world with a commercial nuclear program has a nuclear regulatory body. Examples of efficient and effective regulatory practices should be considered for NRC adoption, as long as it does not comprise NRC's principal mission to protect the health and safety of the public. Particular emphasis should be focused to reduce regulatory review cycle time and decision making and streamline the review process by eliminating low-value added steps.

Staffing (All areas, 25 percent derived) - The NRC and the nuclear energy industry are both faced with addressing similar aging workforce challenges. Of the skills required by NRC staff, many - but not all - are unique to the nuclear energy discipline. Capabilities gained in the industrial sector through optimized training, knowledge management, sustained R\&D, and an increase in the number of students interested in science and technology will augment the NRC potential staff pipeline. In addition, the increased licensing efficiency discussed above will reduce the staffing requirements on a pernuclear-facility basis. 


\section{C.6 Safeguards and Security}

Optimized Technology (All areas, 70 percent derived) —Essentially all of the optimized security technology capabilities required for the nuclear energy industry in the 2010 to 2050 time frame will be developed for other industries. The military and national defense industries have large-scale programs to develop technologies to secure sensitive facilities and protect regions from physical intrusions and attacks. Replacement of the traditional "guns and guards" approach to securing nuclear energy industry facilities (particularly the current 104 commercial power plants) will transition to a technologybased approach requiring fewer humans while providing increased security. Such technologies include high-efficiency electronic surveillance with zero false calls, intruder detection, weapons and explosive detection, and aircraft and watercraft neutralization. The nuclear energy industry should select and deploy security technologies developed for other industries that optimize the securing of all facilities, including reactors and fuel and separation and radioactive material storage facilities.

A unique need of the nuclear energy industry (both commercial power and national defense) is the need to implement nuclear materials accounting systems. A key component of material accountability is advanced sensors, which will be highly specialized and integrated into the basic facility design (as part of "safeguards-bydesign"). This instrumentation will use both radiation-based and non-radiation-based detection, will be online and at-line, and will be based on both passive and active modes of operation. Some fundamental technology development will occur in the nuclear physics $\mathrm{R} \& \mathrm{D}$ arena as well as the homeland security radiation detection arena. However, application in materials accountability requires the highest possible accuracy and reliability, necessitating additional development for use in the nuclear energy industry. Nuclear facilities built and operated within the DOE will create a synergistic eflect that will benefit the needs of the nuclear energy industry in this area.

Data Integration and Control (All areas, 90 percent derived) —Advanced data integration and control systems needed as a critical component of the "safeguardsby-design" implementation will be primarily developed for other industries such as modern manufacturing and large relational database management organizations. The nuclear energy industry will also require near-real-time analysis and decision-making mechanisms, requiring specialization of these general approaches. For example, integration of disparate data will be developed as part of mining internet information, but the quantification of relations within such data with a high degree of accuracy and reliability is an aspect required by the nuclear energy industry to ensure a robust $S \& S$ system.

Cybersecurity (All areas, 100 percent derived) - Similar to optimized security technology, cybersecurity is the focus of major efforts in many industries, including the military. The nuclear energy industry should identify, adapt, and deploy the best cybersecurity approaches that meet NRC, owner/operator, and Department of Homeland Security requirements. Systems requiring protection from cyber threats are not unique to the nuclear energy industry, whether they are wired, optical, or wireless systems. In addition, all efforts should be made in the design process to ensure physical separation of the nuclear facility control systems from other systems that can be breached from the outside, such as the internet and other communication methods. 
Images used courtesy of Battelle, FirstEnergy Nuclear Operating Company, iStockphoto.com, Los Alamos National Laboratory, National Aeronautics and Space Administration (NASA), U.S. Nuclear Regulatory Commission (NRC), and Westinghouse Electric Company, LLC 


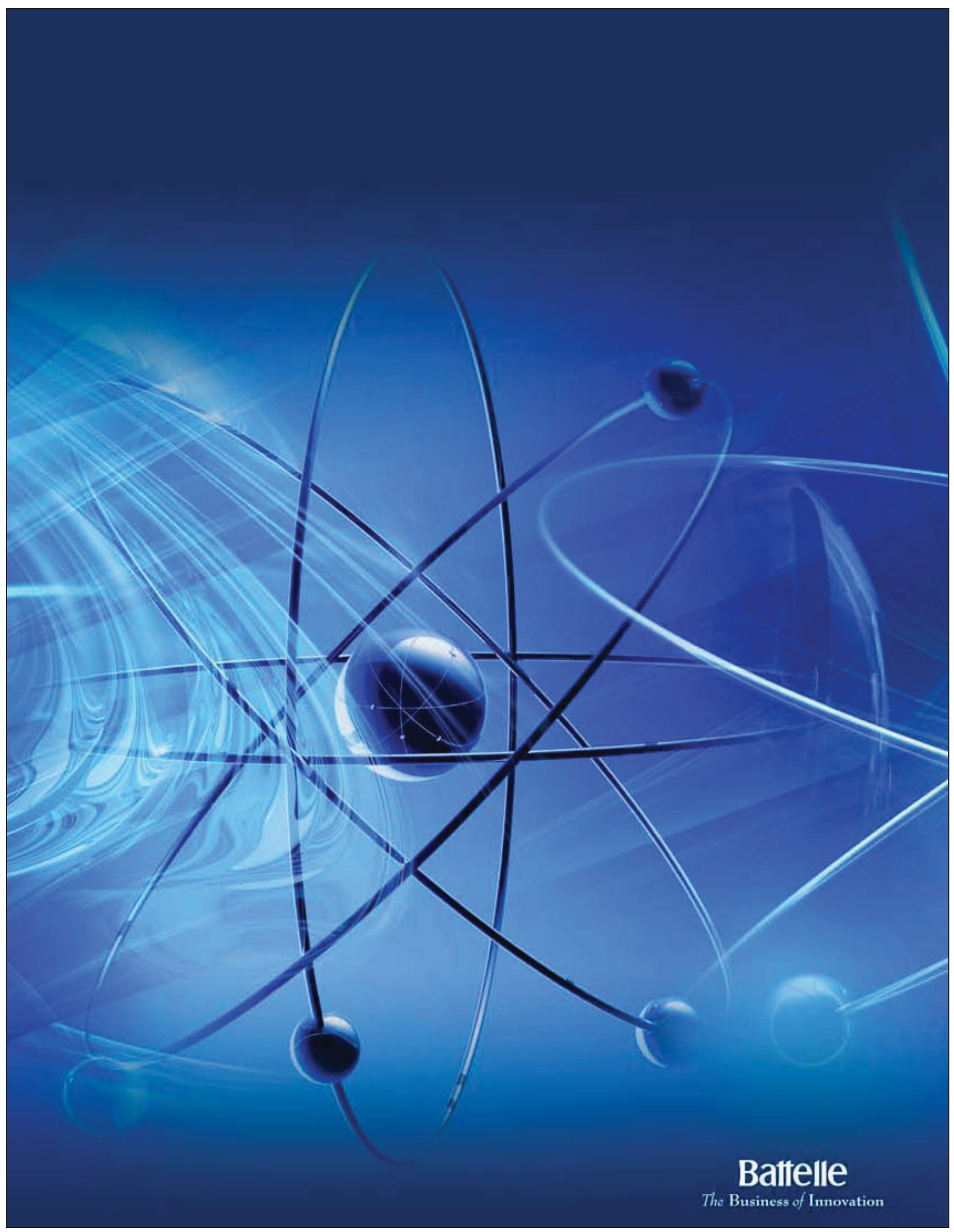




\title{
5.2 Executive Recommendations for Nuclear Research and Development Capabilities
}

\section{Executive Recommendations for Nuclear R\&D Capabilities}

\begin{abstract}
Summary
The energy crisis looming before the United States can only be resolved by an approach that integrates a portfolio of options. Nuclear energy, already an important element in the portfolio, should play an even more significant role in the future as the U.S. strives to attain energy security and reduce carbon emissions. This report identifies the current and future nuclear research and development capabilities required to make this happen. The capabilities support: (1) continued, safe operation of the current fleet of nuclear plants; (2) the availability of a well qualified and trained workforce; (3) demonstration of the next generation nuclear plants; (4) development of a sustainable fuel cycle; (5) advanced technologies for maximizing resource utilization and minimization of waste and (6) advanced modeling and simulation for rapid and reliable development and deployment of new nuclear technologies. In order to assure these capabilities are made available, a Strategic Nuclear Energy Capability Initiative is proposed to provide the required resources during this critical period of time.
\end{abstract}

\section{Scope}

The scope of the recommendations encompasses the commercial use of nuclear energy and what the federal government should do to support its expanded use to increase energy security and reduce carbon emissions. Consequently, the report does not address the production of industrial and medical isotopes, or the use of nuclear energy for space exploration. Nor does the report address the capabilities provided by the commercial nuclear industry, such as low-level waste management. Within its scope, the report does not recommend a specific set of facilities, because this is the responsibility of the DOE through its National Environmental Policy Act and Project Management Order processes. In the context of this report, capabilities mean innovative, wellstructured research and development programs, a viable work force, and well-equipped specialized facilities. These capabilities are targeted investments that that because of their complexity or cost can only be made efficiently and timely by the government and are critically important to our nation's energy policy. Understanding and agreeing upon these investment targets is the essential first step in developing a long-term investment strategy.

\section{Background of Report}

Energy security, energy prices, and the environmental impact of energy production have risen to the top of the national agenda. The United States must increase production capacity, reduce dependence on imported oil, and reduce $\mathrm{CO}_{2}$ emissions. Recent studies project that U.S. energy demand will increase by at least $30 \%$ by the year 2030 . Electricity production, which accounts for one-third of the current man-made $\mathrm{CO}_{2}$ emissions globally, must grow even more rapidly to keep pace with demand even with substantial efficiency improvements.

Safe, reliable, affordable nuclear energy will play an ever increasing role in the generation of base load electricity. Nuclear power generates no $\mathrm{CO}_{2}$ emissions; its life-cycle carbon footprint is negligible. The nuclear industry is working with the U.S. DOE to extend the service life of the 
currently operating reactors, deploy advanced light water reactors as quickly as possible, explore nuclear applications beyond electricity production, develop next generation reactors, and prepare for closing the nuclear fuel cycle.

Achieving these goals will require substantial capabilities that include innovative, wellstructured and adequately funded research and development programs, a viable work force, and well-equipped specialized facilities. Currently only a portion of the required capabilities exists. Developed by a team of senior executives from the nuclear energy industry, academia, and the national laboratories (identified in Appendix A), this Letter Report provides a set of recommendations to address gaps in required capabilities.

\section{The Process}

In early 2008, the U.S. DOE Office of Nuclear Energy requested Battelle work with the domestic nuclear energy industry, the academic community, and the national laboratories to conduct an independent analysis of the capabilities required to support the achievement of the industry's prioritized goals. A three-phase process identified the highest priority capabilities.

In the initial two phases, key studies were conducted to provide the basis for the development of recommendations. The first study, Nuclear Energy for the Future Required RdD

Capabilities -An Industry Perspective was prepared with the participation of 34 organizations representing the nuclear energy industry, academia, and the national laboratories. It articulates the industry's goals for the 2010 to 2050 timeframe and identifies and prioritizes the required capabilities to accomplish these goals. The second report, Assets Required for a Nuclear Energy Applied KdD Program, assesses the availability and state of existing facilities to provide the required capabilities identified in the first study. Some additional DOE NE R\&D program capability requirements beyond those identified in the first study are also addressed in the second study.

In the third phase of the process, the senior executive team reviewed the results of both studies; discussed industry goals, current DOE program activities, and capability gaps; and identified the priorities and recommendations that follow:

- Maximize the continued safe operation of the existing nuclear plants

- Support enhanced nuclear education and training capabilities that complement industry efforts to ensure the availability of a well-qualified and well-trained workforce

- Develop and demonstrate next generation reactor technologies that expand the applications of nuclear energy

- Develop affordable, proliferation-resistant recycle technologies needed to create a sustainable nuclear fuel cycle

- Improve the utilization of uranium resources and reduce waste disposal requirement through the continued development of fast reactor technology

- Utilize advanced modeling and simulation to accelerate the development and deployment of nuclear systems

- Create the Strategic Nuclear Energy Capability Initiative to provide the significantly increased investment in nuclear science and technology capabilities. 
Excellence in science and engineering is the essential foundation for the continuing success of nuclear energy. Recent U.S. investments in nuclear science and engineering education reveal inconsistent implementation of policy and Congressional intent. As its R\&D budget diminished to near zero in the late 1980's, the Office of Nuclear Energy reduced its university support. A new program that showed promising growth was inexplicably abandoned in 2006; since then DOE NE has repeatedly tried to restructure its university program. These attempts have led to confusion and uncertainty throughout the government, industry and academia, resulting in a lack of priority for developing required core competencies. The overall result has been a steady reduction of nuclear education laboratories including on-campus research reactors, and lagging development of teaching and research faculty. Concurrently, the growing demand for graduates in nuclear science and engineering and supporting engineering disciplines has been well documented by such industry and professional organizations as the American Nuclear Society, the American Physical Society, the Health Physics Society, the Electric Power Research Institute and the Nuclear Energy Institute. Consequently, stronger and continuing investments in core competencies are required in the U.S. to realize the promise of nuclear energy, to increase our energy security and reduce our carbon emissions.

Several other federal agencies, including Department of Homeland Security, National Nuclear Security Administration, the U.S. Nuclear Regulatory Commission, and U.S. DOE's Office of Civilian Radioactive Waste Management and Office of Science, support nuclear research and development programs. Opportunities for synergy and cost effective collaboration to provide the needed capabilities for accomplishment of these recommendations are required.

\section{The Recommended Actions}

Maximize the continued safe operation of existing nuclear plants - We recommend DOE NE, in partnership with the nuclear energy industry, undertake a carefully defined and focused R\&D program to help maintain the extended safe operation of the commercial light water reactor fleet while optimizing the power production from each plant for as long as practical.

In 2007, the 104 operating nuclear power plants in the United States produced 20 percent of the country's electricity at the lowest cost, other than hydroelectric. Plant safety, reliability, and economics have improved substantially over the past 29 years, since the Three Mile Island 2 accident. The existing reactor fleet is recognized as one of the pillars of the U.S. electrical energy production infrastructure. The age of the operating plants ranges from 13 to 39 years. Almost $90 \%$ of the operating reactors have secured, or plan to secure, license renewals by the U.S. Nuclear Regulatory Commission (NRC) for an additional 20 years of operation, representing a benefit of hundreds of $\mathrm{SB}$ to the U.S. economy. In addition, extension of life beyond 60 years is under evaluation by the nuclear energy industry and the NRC.

The extended operation of the existing reactors mandates the maintenance of the industry's excellent safety record and high $(>90 \%)$ capacity factors. To achieve these goals continued improvements in plant operations, training, equipment and maintenance are required. Technologies and R\&D investments necessary to extend reactor operation beyond 60 years include improved fuel performance, plant equipment with increased reliability, enhanced materials aging management programs, prognostic systems to optimize component life, and more efficient inspection and repair techniques. Additionally, enhanced systems analysis and integration capabilities are needed to support plant up-rates and optimization of plant systems. A 
successful outcome of these efforts would enable continued safe and cost efficient operations for many years to come. Advanced modeling and simulation of nuclear plant systems will become increasingly important in assessing materials aging, performance optimization, maintaining safety margins, and accelerating regulatory reviews.

Understanding and managing material aging and improving fuel performance are essential when considering extending reactor service life beyond 60 years and to maximize performance, while maintaining the requisite safety performance. Nuclear R\&D facilities including fuel development laboratories, thermal irradiation capabilities, and hot cells for post irradiation examination are required to provide the needed understanding and improvements.

Conclusion - Invest in research and development to further improve and enhance the performance of the existing nuclear plants. Currently available nuclear facilities, with appropriate maintenance and improvements, provide the required facility resources in the near term.

Enhance nuclear education and training facilities to assure the availability of a well qualified and trained workforce - We recommend DOE reassert its legislated role as the steward of nuclear science and engineering education. In this role DOE should ensure that colleges and universities have adequate funding support for nuclear research and specialized nuclear education and R\&D facilities including university research and training reactors. In addition, DOE should coordinate with other federal agencies to ensure the domestic needs for nuclear scientists and engineers are met in an integrated and cost effective manner. Strong academic programs are critical to achieving the excellence in science and engineering which is the essential foundation for the continuing success of nuclear energy in the U.S. Universities with successful programs can play key roles in preparing the next generation of nuclear scientists and engineers to meet the national energy needs.

Education is the foundation for building the core competencies that the U.S. needs if the energy security and environmental goals are to be achieved. The development of a robust nuclear energy workforce is upmost on industry's agenda, and is critically important for our national research infrastructure. Workforce development has to be a shared investment between industry and DOE, with each having responsibility for key areas. Industry has urgent concerns with the building, startup and operation of advanced light water reactors, as well as the replacement of an aging workforce for the existing nuclear plants and programs. This requires skilled craftsmen, technicians and degreed engineers and improved training and development methods. DOE's focus is primarily on developing degreed personnel to ensure the development of needed core competencies in science and technology, which would enhance U.S. competiveness, and sustain a robust research capability.

In prior decades, nuclear engineering enrollments diminished and investment in nuclear education facilities commensurately waned. The latter was highlighted by closure of more than half of the university research reactors. In spite of this erosion of capability, nuclear engineering education is still an area of U.S. leadership, an asset that must be preserved, particularly now that undergraduate enrollments have increased to record levels.

An effective university nuclear science and education program would consist of the following elements: 
1. Basic infrastructure and core competency development - to provide for the development and maintenance of the national educational infrastructure and core competencies through scholarships, fellowships and young faculty and researcher grants.

2. Long-term competitive peer-reviewed university $R \& D$ programs involving research projects that are mission and idea driven - to enable the development of basic technology needed for the next 20 years and beyond.

3. Laboratory and industry driven R\&D programs - to provide significant opportunity for national laboratories, universities, and industry to work together on DOE NE program mission driven R\&D.

Further, DOE is encouraged to join industry, professional organizations, state and local communities in supporting K-12 and trade school education with carefully selected, highly leveraged projects. Great effectiveness can be achieved by linking and leveraging these activities with university nuclear science and engineering programs.

Conclusion - New investment in education and training capabilities is required - further evaluation of specific facility requirements is necessary.

Develop and demonstrate next generation reactor technologies that expand the applications of nuclear energy - We recommend DOE continue developing productive partnerships with industry in order to expand the nuclear market into applications beyond generating electricity. In particular, the immediate opportunity is in supplying process heat for industrial applications. There is also a developing market for regional small to intermediate size reactors. Thanks to technology readiness resulting from previous investments, the U.S. can exercise international leadership in both of these areas if DOE makes them priority goals.

Advanced Light Water Reactors (ALWRs) are excellent for generating electricity. However, if we are to use nuclear energy to help provide other forms of energy, we have to develop and deploy higher temperature reactor systems. The use of water as a coolant restricts ALWRs to about $300 \mathrm{C}$. Process heat applications for refiners and chemical plants require 250 to $550 \mathrm{C}$, oil shale and tar sands processing requires 300 to $600 \mathrm{C}$, electricity and steam cogeneration requires 350 to $800 \mathrm{C}$, reforming of natural gas into hydrogen requires 500 to $900 \mathrm{C}$ while thermochemical processes or high temperature electrolysis of water into hydrogen and oxygen requires 550 to $1000 \mathrm{C}$.

Demonstrating the use of nuclear energy for the production of process heat is an excellent opportunity for U.S. leadership in a developing market that could rival the electricity sector. High-temperature gas (helium) cooled reactors (HTR) have been under development for more than 40 years. Japan, France, China and India all have HTR development programs. The only large scale prototype currently under development is the Pebble Bed Modular Reactor (PBMR) in South Africa, which is designed to use the Brayton cycle to generate electricity. However, to be deployable in the U.S., the HTR technology must be demonstrated in a NRC licensed plant of sufficient size to convince the end users of its viability and potential competitiveness with other forms of energy, particularly natural gas.

The Next Generation Nuclear Plant (NGNP) is a publicly/privately funded partnership to design, license, build and operate a high temperature nuclear demonstration plant that will generate both electricity and hydrogen using process heat to drive a thermo-chemical or high-temperature 
electrolysis water splitting process. The NGNP would be the only large (300 to $400 \mathrm{MWth}$ ) demonstration of a combined electricity/process heat plant. The current DOE program addresses major technical issues such as fuel reliability and structural graphite and regulatory issues such as plant licensing. Engineering development and component test facilities are also needed to support demonstration of the NGNP.

In the past two years, the potential demand for small to intermediate size reactors in the 10 to $600 \mathrm{MWe}$ size seems to be growing in areas that do not have the infrastructure for large ALWRs. While these reactors are thought to be primarily for developing countries, there may be a strong market for intermediate and small reactors in the less populated parts of the U.S. DOE should explore this need through a program like NP-2010 letting the industry take the lead on development, licensing, and deployment.

Conclusion - Provide continuing and sufficient federal resources to the NGNP and its supporting technologies to meet the public obligations of the partnership and assist with the licensing of first of a kind small to intermediate size reactors.

Develop the affordable, proliferation-resistant recycle technologies needed to create a sustainable fuel cycle - We recommend DOE: (1) maintain a substantial R\&D program to develop the technologies required to implement a sustainable nuclear fuel cycle in the U.S., (2) increase university support to build a viable workforce, particularly in the field of radiochemistry, (3) implement a short-term strategy to increase the extent of collaborative R\&D programs with those countries currently recycling nuclear fuel, including Japan, France and the $\mathrm{UK}$, and (4) work with industry to develop improved, commercially viable technologies. In support of these recommendations, DOE should maximize the use of available domestic facilities for the development of a sustainable closed nuclear fuel cycle.

The current open nuclear fuel cycle discards the majority of the potential energy of the uranium as radioactive waste, a practice that may not be sustainable in light of the anticipated large global expansion of nuclear energy in the $21^{\text {st }}$ century. The Yucca Mountain geologic repository for used nuclear fuel is at an early stage of license review and will not be available for many years. Used nuclear fuel is currently stored at the various utility reactor sites. The utilities expect to move used nuclear fuel offsite based on their existing agreement with the U.S. government. The used fuel can be safely stored for decades, so there is no immediate urgency to reprocess the fuel or put it in a deep geological repository. Nevertheless, there is an urgency to proceed with an interim storage plan that assures the public and the utilities that there is a national solution for the management of used nuclear fuel.

While there is no immediate uranium resource limitation, foreign experience has shown that development of affordable recycling technologies with robust, designed-in anti-proliferation features will likely take decades to develop and implement. It is in the security interest of the U.S. to remain highly engaged with the international community on the development and implementation of the sensitive technologies of the nuclear fuel cycle. Recycling nuclear fuel is practiced by several foreign countries, but the facilities require a significant (billions of dollars) upfront capital investment. Research is needed on methods to: make used fuel recycle more economical; develop advanced nuclear fuels; provide better methods of waste management; and develop and demonstrate new technologies for non-proliferation. These efforts require special 
facilities that can be operated safely while allowing research staff to work with highly radioactive materials

Over the last 30 years, many of the fuel cycle research and development facilities have been shut down due to lack of funds for sustained research and increased costs for maintenance and regulatory compliance. A few facilities are still operating in the national laboratory system which should continue to be used for R\&D efforts supporting a sustainable nuclear fuel cycle, although significant investments will be required to meet $21^{\text {st }}$ century research, safety standards and safeguards requirements.

For the longer-term, the concept of an Advanced Fuel Cycle Facility that incorporates all the necessary capabilities should continue to be evaluated. A decision is needed as to whether these capabilities should be distributed, or consolidated into a single new facility or some combination of upgraded facilities and new construction. A careful analysis is recommended to determine a cost-effective strategy that minimizes the number of high security facilities and optimizes the use of existing capabilities.

Conclusion - Additional investment in domestic facilities and collaborative use of foreign facilities is necessary to provide the capabilities to develop technologies for closure of the fuel cycle. Near-term fuel cycle $R \& D$ activities should maximize the use of currently available facilities.

Improve the utilization of uranium resources and reduce waste disposal requirements through the continued development of fast reactor technology - We recommend developing fast reactor technology by building on our fast reactor core competencies and industrial development experience, enhancing cost-shared international collaboration, and ultimately a licensed prototype.

The U.S. is losing its expertise in fast reactor technology developed in the 1970s and 1980s. With the expected global surge of new nuclear installations, fast reactors are likely to emerge as a major sector of the nuclear mix because of their unique potential for resource utilization and waste management. Having closed major elements of its fast reactor development infrastructure such as the Experimental Breeder Reactor-II and the Fast Flux Test Facility, DOE should consider an interim strategy of rebuilding core competency using existing capabilities and relying on participation in fuel irradiation and other testing in international facilities.

Fast reactors are likely to have a central role in sustaining the nuclear fuel cycle later in the $21^{\text {st }}$ century. They will convert troublesome actinides into high-quality energy sources and readily managed short-lived fission products. The technology supporting design of sodium-cooled fast reactors (SFR) is extensive, the result of over 50 years of R\&D in the U.S. and other countries. Active SFR development programs are under way in China, France, India, Japan, South Korea and Russia. The U.S., France and Japan have recently signed a trilateral agreement for sharing the development of SFR technology. The global interest in SFR provides an excellent opportunity for the U.S. to leverage its significant investment in fast reactor technology development through strengthened international collaborations. It is further recommended that the U.S. provide technical leadership for SFR development by establishing a recognized core competency in several critical technology areas such as advanced fuels, safety, and power conversion. 
Conclusion -Combining recognized core competencies in critical areas with a robust program of international collaboration will position the U.S. to move forward with its own licensed prototype demonstration when appropriate.

Utilize advanced modeling and simulation to accelerate the development and deployment of nuclear systems - We recommend DOE NE develop a robust nuclear modeling and simulation capability to accelerate the development and deployment of nuclear energy systems. Capabilities required include strengthened core competencies, modern software, high-performance computing, preservation of unique integral test data, and specialized capabilities for single effects testing.

Nuclear science and technology has not taken advantage of advances in modeling and simulation capabilities that have benefitted other technologies and scientific disciplines. While modeling and simulation does not replace the need for experiments, it can be used to inform the design of experiments, make better use of experimental data, greatly reduce development cycle length, and quantify safety and performance margins. Advanced modeling and simulation is an essential crosscutting tool for improving the performance of current reactors, and for the development and licensing of next generation nuclear reactors and improved fuel recycle technologies. We recommend that DOE NE at a minimum take the following four steps in the near term to accelerate the application of advanced modeling and simulations to nuclear energy systems:

1. Improve access to existing advanced modeling and simulation capabilities. DOE through the NNSA and Office of Science has extensive capabilities in advanced modeling and simulation. An initiative should be undertaken to provide quick access to the existing capabilities for application to nuclear energy systems.

2. Define the architecture needed for the advanced modeling and simulation of nuclear energy systems. Understanding the needed architecture (hardware, software, core competencies) over the development life cycle of advanced nuclear systems will provide an understanding of the investment required. The nuclear energy industry should participate as a user of such capabilities in the definition of the required architecture.

3. Establish a pilot program to demonstrate the value added by advanced modeling and simulation for improving the performance and operation, in particular materials aging management, of the current nuclear plants, for the design of new nuclear systems, safety analysis and licensing.

4. Quickly establish a low-cost, high-value program to document, validate and archive data from prior large scale experiments that would be impossible to repeat today. At minimum, an archive should be developed and maintained for physics, thermal-hydraulic, safety, fuels, and materials data. The archive should include any available test samples.

Conclusion - Develop a robust nuclear modeling and simulation capability to accelerate the development and deployment of nuclear energy systems.

Establish a new initiative for investing in required nuclear energy capabilities - We strongly recommend a Strategic Nuclear Energy Capability Initiative be established to provide the significantly increased investment needed to reestablish an internationally viable U.S. nuclear science and technology infrastructure. 
Funds provided through the Initiative would be used to build core competencies, foster collaborations between industry, academia, and the national laboratories, enhance capabilities, maintain and modify currently available facilities and provide new R\&D facilities and associated equipment. This Initiative would also provide the financial foundation for DOE NE participation in public-private partnerships and international collaborations to leverage U.S. taxpayer investment in required capabilities and facilities. To be successful, the Strategic Nuclear Energy Capability Initiative must have the following attributes:

1. Utilize an integrated, time-phased, and user-driven approach. The initial focus would be on building core competency in nuclear science and engineering, procurement of needed research equipment, improvement in current R\&D facilities, and development of userdriven concepts for needed new R\&D facilities.

2. Provide multi-year investments in a manner that enables efficient development of capabilities, commissioning of the needed facilities, and decommissioning of obsolete facilities.

3. Engage the nuclear energy industry, universities, and the national laboratories in the development and evaluation of user-driven concepts to provide the rationale for the investment to build over time to enable the construction of the new R\&D facilities. Integrated periodic reviews of the Initiative with the user of the capabilities and facilities should be conducted to help ensure the investment is yielding the desired results.

Conclusion - Establish the Strategic Nuclear Energy Capability Initiative to provide the required resources during this critical period of time to assure the proper utilization of nuclear energy. 


\section{Recommendations}

Based upon this independent analysis of the required R\&D capabilities in the 2010 to 2050 timeframe the following recommendations are made.

1. Invest in research and development to further improve and enhance the performance of the existing nuclear plants. Currently available nuclear facilities - with appropriate maintenance and improvements - provide the required research capabilities for extending the safe and useful life of the existing nuclear fleet

2. New investment in education and training capabilities is required to assure the availability of a well-qualified and trained workforce. Further evaluation of specific facility requirements is necessary.

3. Provide continuing and sufficient federal resources to the NGNP and it supporting technologies to meet the public obligations of the partnership and assist with the licensing of first-of-a-kind small to intermediate size reactors to develop and demonstrate next generation reactor technologies that expand the applications of nuclear energy.

4. Additional investment in domestic facilities and the collaborative use of international facilities will be necessary to address all of the activities necessary to create a sustainable fuel cycle. Near-term fuel cycle R\&D activities should optimize the use of currently available facilities.

5. Combine recognized fast reactor core competencies in critical areas with a robust program of international collaboration to position the U.S. to move forward with its own licensed prototype demonstration when appropriate in order to improve the utilization of uranium resources and reduce waste disposal requirements.

6. Develop a robust nuclear modeling and simulation capability to accelerate the development and deployment of nuclear energy systems.

7. Establish the Strategic Nuclear Energy Capability Initiative to provide the required resources during this critical period of time to assure the proper utilization of nuclear energy. 


\section{Appendix A}

\section{Executive Team Participants}

Don Hintz, Entergy

Jim Duderstadt, Univ. of Michigan

Kenneth L. Peddicord, Texas A\&M University

Regis Matzie, Westinghouse

Tom Christopher, AREVA

Joe Turnage, Constellation/UniStar

Rich Reimels, B\&W

Tom Hunter, Sandia

John Grossenbacher, INL

Thom Mason, ORNL

Jeff Wadsworth, Battelle

Harold McFarlane, INL

Dana Christensen, ORNL

Paul Kearns, Battelle

\section{DOE Observers}

Dennis Spurgeon

Dennis Miotla

Tim Frazier 


\subsection{Reviewer Supplemental Information and Attachments}

After publishing the first draft of this report (September 2008), an interactive web edition was placed on the internet and advertised internationally to stakeholders requesting their review and input. The interactive web edition was available for review during the last quarter of 2008. That review resulted in more than 150 comments and supplemental inputs from reviewers across the DOE complex, in industry, and academia. While the comments and supplemental information potentially would add significant value to a future study, this project is limited to the perspective that was developed in 2008 .

The comments and supplemental information that were received have been included in one of two ways in this final report. The written comments have been summarized and included at the end of the appropriate section. Verbatim comments were not used in order to eliminate redundancy, reduce bulk, and to protect the privacy of the reviewers. However, the verbatim comments were displayed on the review web site during the open review period.

Several reviewers supplied supplemental information such as charts and tables that they felt should have been included in the report. This material has been summarized below. No attempt has been made to validate the material; that is left to a future activity.

\subsubsection{Supplemental Information Included in this Report}

- Sandia's Annular Core Research Reactor (ACRR) was included in several sections of this report, but was inadvertently omitted from Section 3.3, Domestic Facility Descriptions. ACRR has now been included in Section 3.3.

\subsubsection{Supplemental Information Submitted and Filed for Future Consideration}

- $\quad$ ANL submitted a nine-page white paper entitled CSNI Task on Advanced Reactor Experimental Facilities (Infrastructure for Safety Research) - DOE Laboratory Facilities Input for Gas Reactor Research Questionnaire, a compilation of responses to questions about applicable facility capabilities and issues

- $\quad$ ANL Radiochemical Sciences and Engineering Facilities (radiological laboratory space located in three ANL buildings) description, status, and potential program R\&D applications

- $\quad$ ANL Hot Cell Facilities - proposed revisions to existing ANL J, G, K and M-Wing and other ANL facility descriptions, statuses, capabilities, condition, availability, etc., information in the report

- $\quad$ ANL Irradiated Materials Laboratory (IML) facility description, status, and potential program R\&D applications

- $\quad$ ANL Alpha-Gamma Hot Cell Facility (AGHCF) facility description, status, and potential program R\&D applications

- $\quad$ ANL Advanced Photon Source (APS) facility description, status, and potential program R\&D applications

- $\quad$ ANL Building 212 Materials Testing Laboratory (MTL) facility description, status, and potential program $R \& D$ applications 
- $\quad$ ANL Laboratory Computing Resource Center (LCRC) facility description, status, and potential program R\&D applications

- $\quad$ ANL Argonne Leadership Computing Facility (ALCF) facility description, status, and potential program R\&D applications

- ANL proposed changes to the assessed program support capability/applicability and the assessment of attributes (condition, capability, availability, etc.) for a set of ANL facilities existing in the report and proposed for the report

- $\quad$ ORNL Oak Ridge Electron Linear Accelerator (ORELA) facility description, status, and potential program R\&D applications

- $\quad$ ORNL Uranium Fuel Development Laboratory facility description, status, and potential program R\&D applications

- $\quad$ ORNL Radiochemical Analysis Group (a group of three radiological analytical laboratories) description, status, and potential program R\&D applications

- $\quad$ ORNL Core Conduction Cool-Down Test Facility (CCCTF) facility description, status, and potential program R\&D applications

- $\quad$ ORNL Radiochemical Engineering Development Center buildings 7920 (REDC 7920) and 7930 (REDC 7930) are included in the report as a single facility, but were resubmitted as two separate facilities with distinct facility descriptions, statuses, and potential program R\&D applications

- $\quad$ Sandia Gamma Irradiation Facility (GIF) facility description, status, and potential program R\&D applications 


\subsection{Contributors to the Report}

\subsubsection{Writing and Production}

$\begin{array}{ll}\text { Harold McFarlane } & \text { Idaho National Laboratory } \\ \text { John Sackett } & \text { Consultant } \\ \text { Bruce Matthews } & \text { Consultant } \\ \text { Craig Jacobson } & \text { Idaho National Laboratory } \\ \text { Robert Phipps } & \text { Idaho National Laboratory } \\ \text { Andrew Klein } & \text { Idaho National Laboratory }\end{array}$

\subsubsection{Workshop Participants}

$\begin{array}{ll}\begin{array}{ll}\text { Harold McFarlane, Lead } \\ \text { Robert Wham }\end{array} & \begin{array}{l}\text { Idaho National Laboratory } \\ \text { Oak Ridge National Laboratory }\end{array} \\ \text { Paul Kearns } & \text { Battelle } \\ \text { James Buelt } & \text { Pacific Northwest National Laboratory } \\ \text { Chris Grandy } & \text { Argonne National Laboratory } \\ \text { John Marra* } & \text { Savannah River National Laboratory } \\ \text { John Ireland } & \text { Los Alamos National Laboratory } \\ \text { Kimberlyn Mousseau } & \text { Idaho National Laboratory } \\ \text { Andrew Klein } & \text { Idaho National Laboratory } \\ \text { George Imel } & \text { Idaho State University } \\ \text { *Via teleconference } & \end{array}$

\subsubsection{National Research and Development Directors}

Terry Todd Separations

Kemal Pasamehmetoglu Fuels

David Petti NGNP

Ronaldo Szilard LWR Sustainability 


\subsubsection{Department of Energy Support}

Extensive input was provided by DOE program managers through reports and presentations.

Dennis Miotla

Paul Lisowski

Rob Versluis

Daniel Stout

Carl Sink
Facility Infrastructure

Global Nuclear Energy Partnership

NGNP

Separations

Nuclear Hydrogen Initiative 
INL/EXT-08-14092 